\title{
TYPE II ACTIVATION OF MONOCYTES IN MULTIPLE SCLEROSIS
}

\author{
By \\ Delgertsetseg Chuluundorj
}

A thesis submitted to the Victoria University of Wellington in the fulfilment of the requirements for the degree of

Doctor of Philosophy

Victoria University of Wellington

2013 


\begin{abstract}
Multiple sclerosis (MS) is an incurable autoimmune disease of the CNS. Although its cause is not known, immune cells are involved in the disease progression. Among these cells, type I monocytes are first to arrive to the brain and initiate inflammation; however, if monocytes are type II activated, they can inhibit inflammation. Previous research has shown that immune responses can be modulated by treatments, such as glatiramer acetate (GA) and immune complexes (IC). Therefore, we aimed to determine whether GA and IC can induce type II activation of monocytes in MS.
\end{abstract}

Human blood monocytes from healthy volunteers and MS patients were stimulated in vitro with bacterial lipopolysaccharide (classical activation) in the presence or absence of GA and immune complexes (IC) composed of IVIG and human red blood cells (type II activation). Flow cytometry, ELISA and cytometric bead array were used to assess levels of marker expression and cytokine production in order to define the activation of monocytes.

Interestingly, while both GA and IC induced type II activation of monocytes, the characteristics of these type II monocytes were distinct. We have found that monocytes from both healthy people and MS patients have significantly lower levels of inflammatory marker CD40 and higher levels of the anti-inflammatory cytokine IL-10 after treatment with IC. In contrast, GA treatment reduced the levels of CD40, CD86 and the inflammatory cytokine IL12. Moreover, the combined addition of GA and IC appeared to be more effective in type II activating monocytes than either agent alone. We also found that both $\mathrm{CD} 14^{++} \mathrm{CD} 16^{-}$and $\mathrm{CD} 14^{+} \mathrm{CD} 16^{+}$monocyte subsets can be type II activated by the treatments; however, an interaction between the subsets impaired their response to the treatments.

Our study suggests that treatments with GA and IC, especially in combination, are effective in type II activation of human monocytes and can be beneficial therapeutic approaches for multiple sclerosis. 


\section{Acknowledgments}

First, I would like to express my sincere gratitudes to my supervisor, Dr. Anne Camille La Flamme, for her enormous support and intelligent guidance through the years of my $\mathrm{PhD}$ study. She has been a great mentor and has showed me how one can be developed from a "child" to a doctor. Having all the best qualities of a supervisor, such as patience, wisdom, knowledge, acceptability, kindness and a lot more, she has become my role model for the entire life. And, I would like to thank her for all her priceless efforts, strengthening encouragements, assistance, inspirations, sleepless nights and restless holidays that she has spent for me during my $\mathrm{PhD}$ study. Also, I would like to acknowledge my secondary supervisor Dr. Scott Harding for his time and efforts.

I can not imagine my laboratory life without a group of wonderful young scientists that has been my second family at the VUW SBS laboratory. I would like to acknowledge the LAF group, including Sara Mirmoeini, David O’Sullivan, Marie Kharkrang, Maddie White, Ryan Kyle, Bhagyashree Manivannan, Nikki Templeton, Lisa Johnston, Laura Green, Hanane Belhoul and Jenni Williams; Dr. John Miller and his group, including Ariane Chang, Jessica Field, Viswanath Das, Zlatka Smart and Arun Kanakkanthara, for all their support and warm friendship during my academic life. I also thank Jenni Williams, Jessica Field and Ariane Chang for the efficient proofreading of my thesis; and Janet Pitman, Arun Kanakkanthara and Ryan Steel for their invaluable help in my PCR experiments. Lastly, I am grateful to everyone in the VUW Alan McDiarmid building level 3 laboratories for making my PhD study such an exciting, happy and memorable period of my life that I will cherish for all my life.

I would like to acknowledge my sons Tuvshinbaatar B. and Tuvshin-Orgil B., who has been my light and belief in the future; and my parents, professors Chuluundorj B. and Tseyen-Oidov Ts. for raising me with an affection for education. I am grateful to all my Mongolian friends, relatives and colleques for believing in me and my achievements.

Finally, I would like to deeply thank my husband Bayasgalan $\mathrm{Ch}$. for his tremendous support, sacrifices, encouragements, trust and wisdom that has played the major role behind the scenes of my academic life. 


\section{Table of contents}

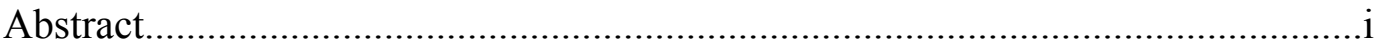

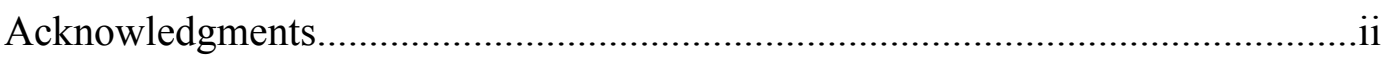

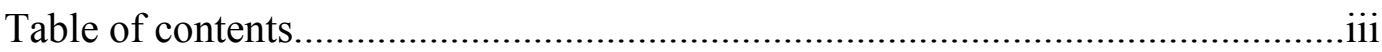

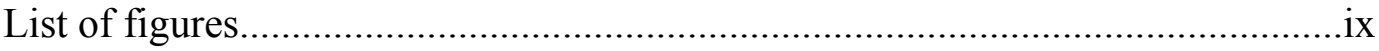

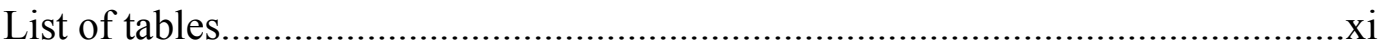

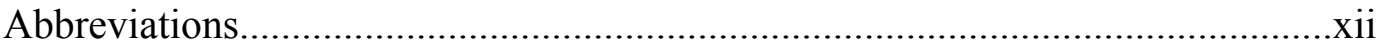

Chapter 1. General introduction......................................................1

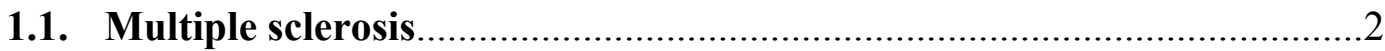

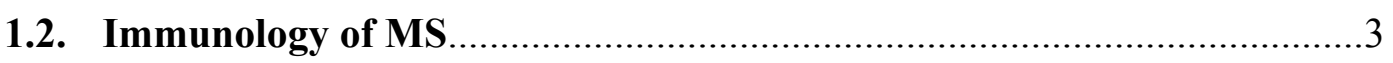

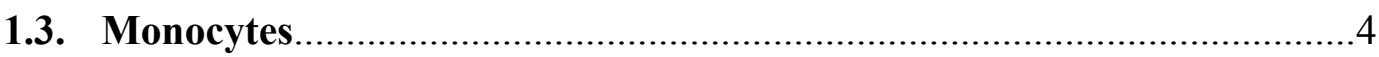

1.3.1 Monocytes as immune cells....................................................

1.3.2 Characteristics and function of monocytes.................................4

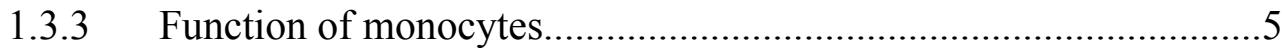

1.3.4 Classical activation of monocytes................................................

1.3.5 Type II activation of monocytes ................................................6

1.3.6 Cell surface markers and cytokines in monocytes.........................6

1.3.7 Role of monocytes in inflammation and MS.............................. 7

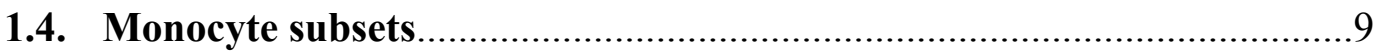

1.4.1 $\mathrm{CD}^{+} 4^{+}$and $\mathrm{CD} 16^{+}$monocyte subsets.......................................

1.4.2 $\mathrm{CD}^{+} 4^{+}$and $\mathrm{CD} 16^{+}$monocytes in inflammation and MS................9

1.4.3 Activation of $\mathrm{CD}_{14}{ }^{+}$and $\mathrm{CD}_{1} 6^{+}$monocytes................................10

1.4.4 Role of subsets in brain inflammation and MS.............................11

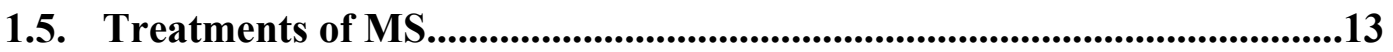

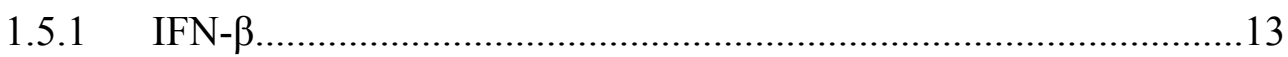

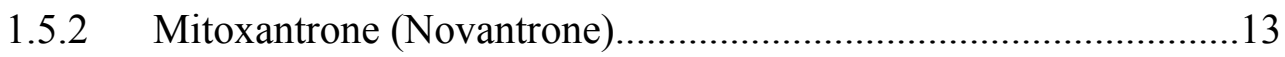

1.5.3 Fingolimod (Gilenya)........................................................... 14

1.5.4 Natalizumab (Tysabri) ...............................................................14

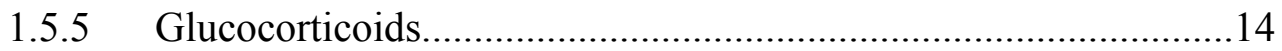

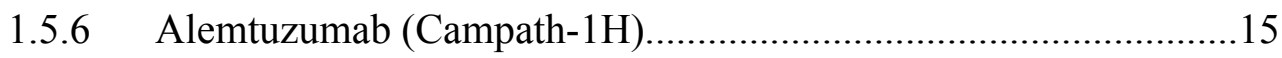

1.5.7 IVIG (intravenous immunoglobulin G)...................................15

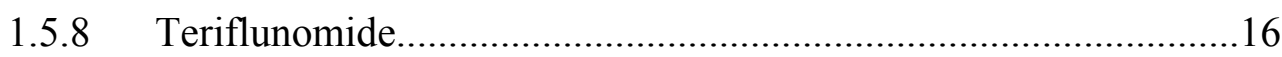




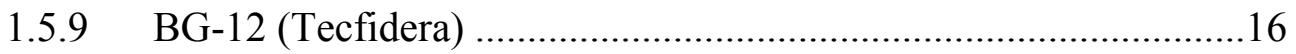

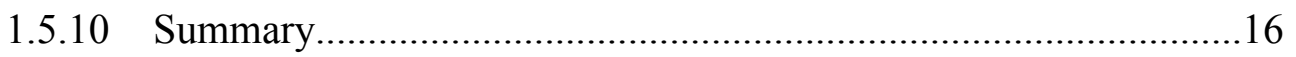

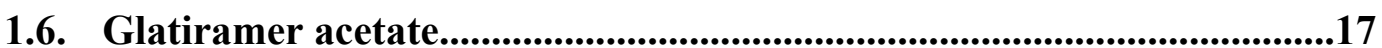

1.6.1 Glatiramer acetate in MS ..........................................................17

1.6.2 Effect of glatiramer acetate on monocytes and their subsets..........18

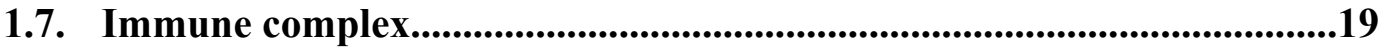

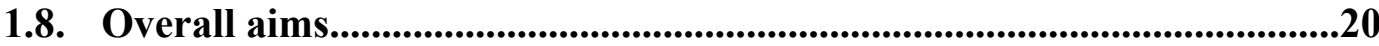

Chapter 2. Materials and methods.........................................................21

2.1. Subjects and blood samples......................................................................22

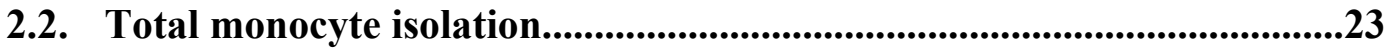

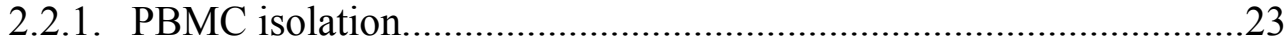

2.2.2. Isolation of total monocytes from PBMC.....................................23

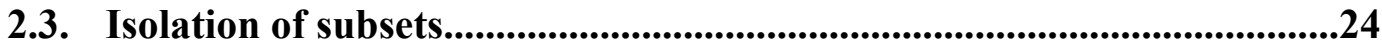

2.3.1. Depletion of NK cells and granulocytes........................................24

2.3.2. Positive isolation of $\mathrm{CD}_{1} 6^{+}$monocytes.........................................24

2.3.3. Positive isolation of $\mathrm{CD} 14^{+}$monocytes........................................24

2.4. Monocyte culture and IFN- $\gamma$ priming...........................................................24

2.5. LPS stimulation..................................................................................................25

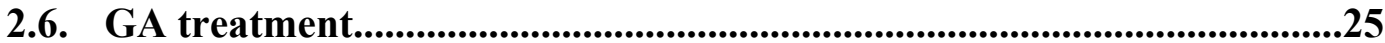

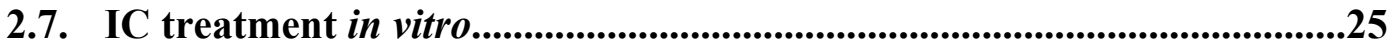

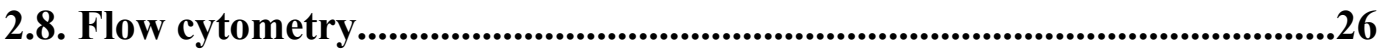

2.8.1 Staining and data acquisition...................................................26

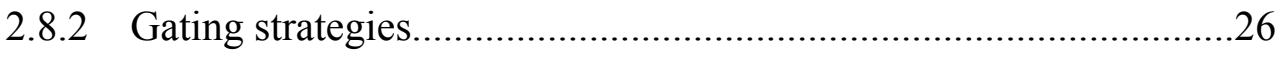

2.9. Cytokine measurement....................................................................................26

2.9.1 Extracellular cytokine detection by ELISA.................................26

2.9.2 Extracellular cytokine detection by cytometric bead array...........27

2.9.3 Intracellular cytokine detection by flow cytometry...........................28

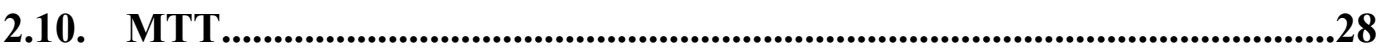

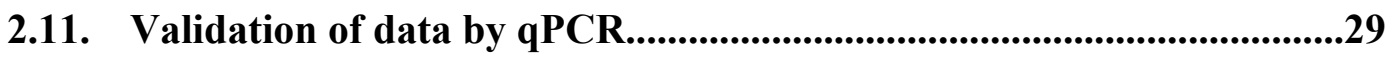

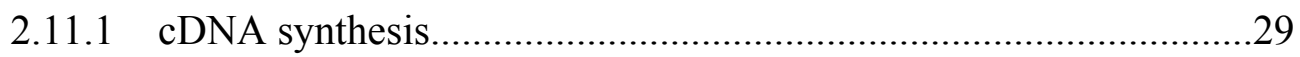

2.11.2 Quantitative real-time PCR ........................................................29

2.12. Hierarchial cluster analysis......................................30 
Chapter 3. Characterization of ex vivo monocytes from MS patients...31

3.1. Introduction........................................................................................................32

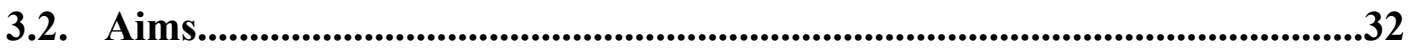

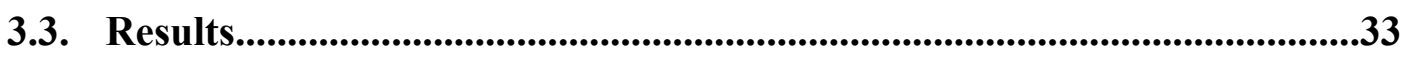

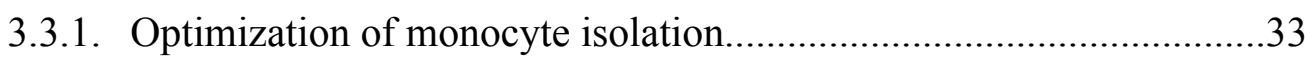

3.3.1.1. Isolation of total monocytes.............................................33

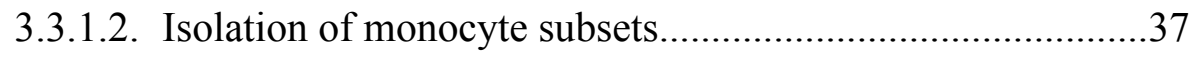

3.3.2. Proportion of monocytes from healthy and MS groups.....................41

3.3.3. Activation state of monocytes from healthy and MS groups..............41

3.3.4. Comparison between $\mathrm{CD} 14^{+}$and $\mathrm{CD} 16^{+}$subset phenotypes..............45

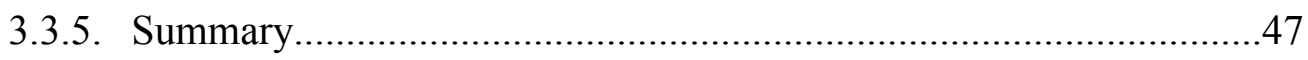

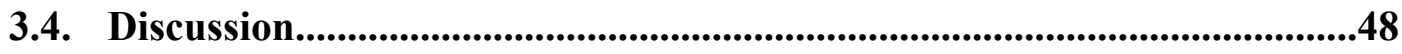

3.4.1. Characteristics of monocytes and their subsets..............................48

3.4.2. Proportion of monocyte subsets in MS patients..............................48

3.4.3. Expression of activation markers in monocytes in MS...................49

Chapter 4. Type II activation of total monocytes in vitro..................53

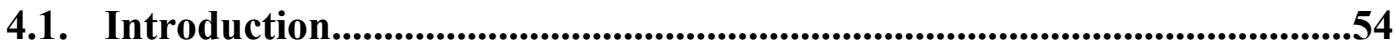

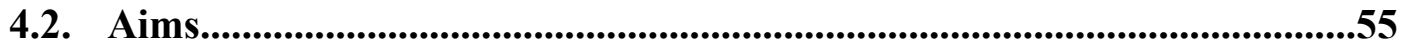

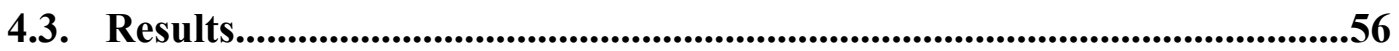

4.3.1. Optimization of monocyte culture and treatments........................56

4.3.1.1. Optimization of classical activation.................................56

4.3.1.2. Optimization of GA treatment........................................58

4.3.1.3. Optimization of IC treatment...........................................59

4.3.2. Classical activation of monocytes...............................................62

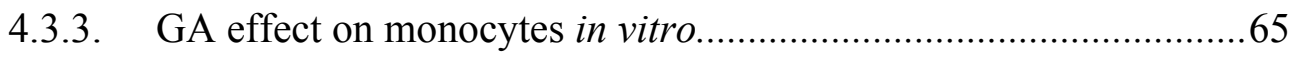

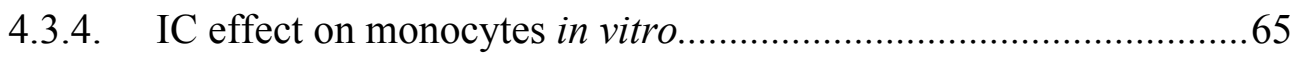

4.3.5. The effect of combination treatment on monocytes in vitro...........66

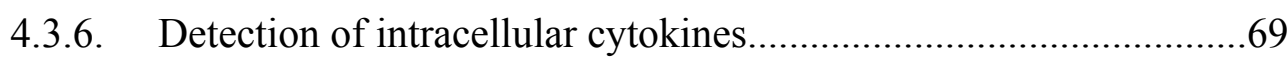

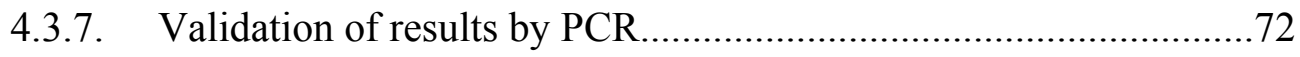

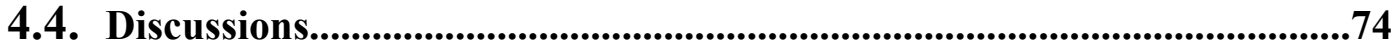


4.4.1. In vitro classical activation of monocytes in MS...............................74

4.4.2. In vitro GA effect on monocytes in MS.............................................74

4.4.3. In vitro IC effect on monocytes in MS.............................................. 75

4.4.4.In vitro IC and GA effect on monocytes in MS................................78

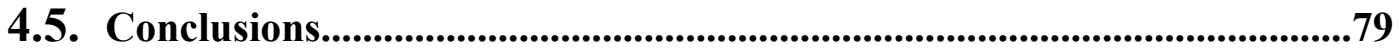

\section{Chapter 5. Type II activation of $\mathrm{CD}^{+} 4^{+}$and $\mathrm{CD} 16^{+}$monocyte subsets...80}

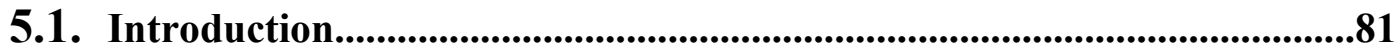

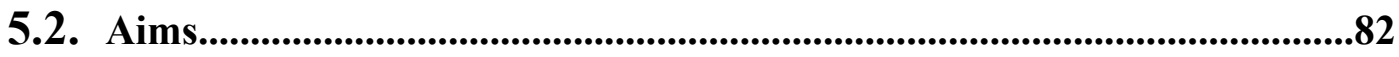

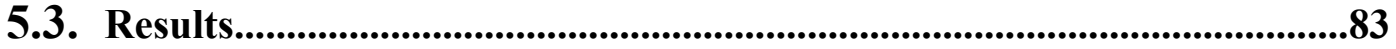

5.3.1. Classical activation of $\mathrm{CD} 14^{+}$and $\mathrm{CD} 16^{+}$monocytes in vitro.......83

5.3.1.1. Classical activation of $\mathrm{CD} 14^{+}$monocytes .......................83

5.3.1.2. Classical activation of $\mathrm{CD} 16^{+}$monocytes.........................85

5.3.1.3. Comparison between the classical activation of $\mathrm{CD} 14^{+}$ and $\mathrm{CD} 16^{+}$monocytes.................................................87

5.3.2. The effect of $\mathrm{GA}$ on $\mathrm{CD} 14^{+}$and $\mathrm{CD} 16^{+}$monocytes in vitro...........91

5.3.2.1. The effect of GA on $\mathrm{CD} 14^{+}$and $\mathrm{CD} 16^{+}$monocytes..........91

5.3.2.2. Comparison of the GA effect between $\mathrm{CD} 14^{+}$

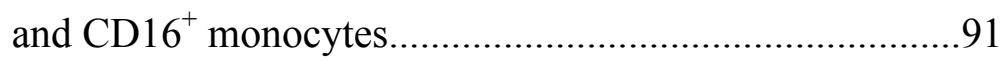

5.3.3. The effect of $\mathrm{IC}$ on $\mathrm{CD} 14^{+}$and $\mathrm{CD} 16^{+}$monocytes in vitro.............92

5.3.3.1 The effect of IC on $\mathrm{CD} 14^{+}$and $\mathrm{CD} 16^{+}$monocytes ..........92

5.3.3.2 Comparison of the IC effect between $\mathrm{CD} 14^{+}$and $\mathrm{CD} 16^{+}$

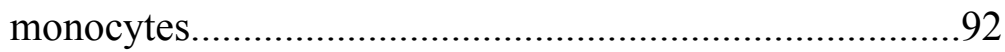

5.3.4. The effect of combination treatment on subsets in vitro................93

5.3.4.1 The effect of GA and IC on $\mathrm{CD}_{14}{ }^{+}$and $\mathrm{CD} 16^{+}$subsets...93

5.3.4.2 Comparison of the GA and IC effect between

$\mathrm{CD}_{14}{ }^{+}$and $\mathrm{CD} 16^{+}$monocytes

5.3.5. The effect of subset interactions on the activation of

$\mathrm{CD} 14^{+}$and $\mathrm{CD} 16^{+}$monocytes .97

5.3.5.1 Classical activation of co-cultured subsets.......................97

5.3.5.2 The effect of GA on co-cultured subsets..........................98

5.3.5.3 The effect of IC on co-cultured subsets............................99

5.3.5.4 The effect of combination treatment on co-cultured subsets...99 
5.4.1. Classical activation of $\mathrm{CD} 14^{+}$and $\mathrm{CD} 16^{+}$monocytes in vitro........105

5.4.2. The effect of GA on $\mathrm{CD} 14^{+}$and $\mathrm{CD} 16^{+}$monocytes in vitro...........107

5.4.3. The effect of IC on $\mathrm{CD} 14^{+}$and $\mathrm{CD} 16^{+}$monocytes in vitro............. 108

5.4.4. The effect of GA and IC combination treatment on monocyte subsets in vitro.

5.4.5. Interaction between the subsets: response to the treatments in co-cultures.

Chapter 6. Type II activation of monocytes by GA in vivo..........114

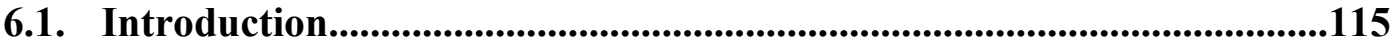

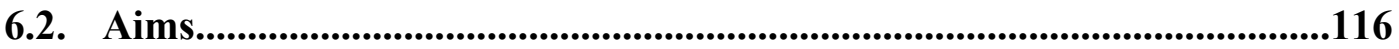

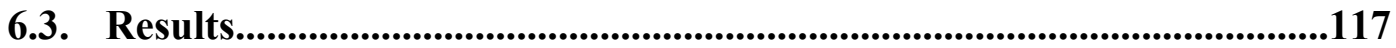

6.3.1. Proportion of subsets from in vivo GA-treated MS patients.

6.3.2. Type II activation of monocytes and their subsets by in vivo

GA treatment.

6.3.3. Comparison of in vivo and in vitro GA effect on monocytes and their subsets.

6.3.4. Classical activation of in vivo GA-treated monocytes

6.3.5. In vitro GA effect on in vivo GA-treated monocytes and subsets...125

6.3.6. In vitro IC effect on in vivo GA-treated monocytes and subsets.....125

6.3.7. Difference in monocyte activation between the subject groups......128

6.3.8. Comparison of monocyte activation between the subject groups after in vitro treatments. 130

6.4. Discussion .135

6.4.1. Proportion of monocytes from in vivo GA-treated MS patients......135

6.4.2. In vivo GA effect on monocytes and their subsets. 136

6.4.3. Comparison of in vivo and in vitro GA effect on monocytes and their subsets

6.4.4. Classical activation of in vivo GA-treated monocytes and their subsets.

6.4.5. In vitro $\mathrm{GA}$ and $\mathrm{IC}$ effect on in vivo GA-treated monocytes and 
their subsets

6.4.6. Comparison of monocyte activation between the subject groups....139

6.5. Conclusion..

Chapter 7. General discussion.

7.1. Outcome of results: the role of monocytes in the pathogenesis of MS..142

7.2. Outcome of results and clinical significance: GA effect in MS..............144

7.3. Outcome of results and clinical significance: IC effect in MS................146

7.4. Future directions........................................................................................147

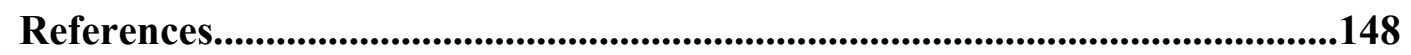

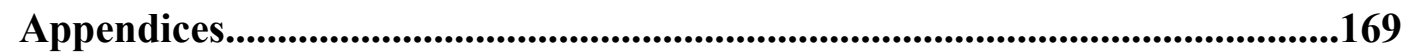

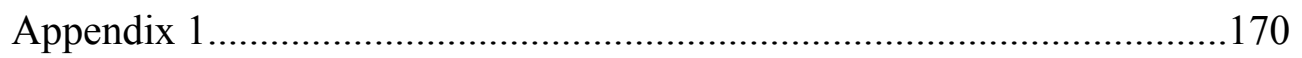

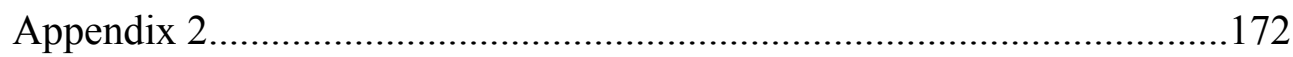

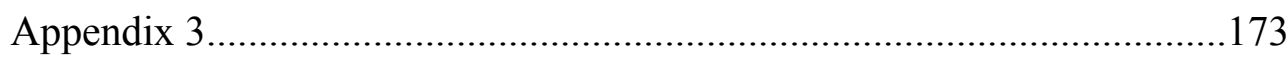

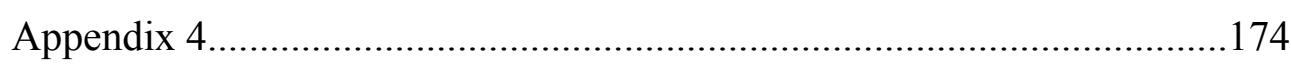

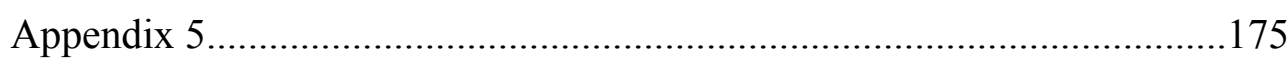

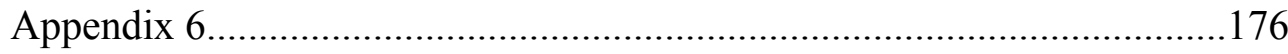

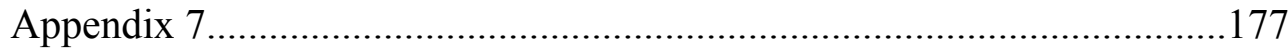

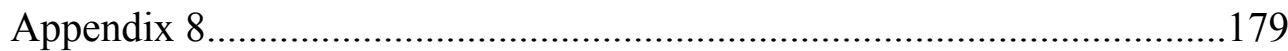

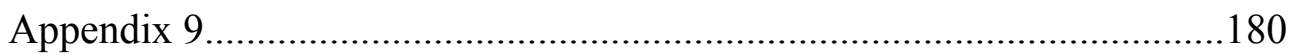

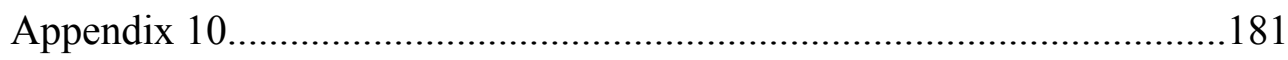

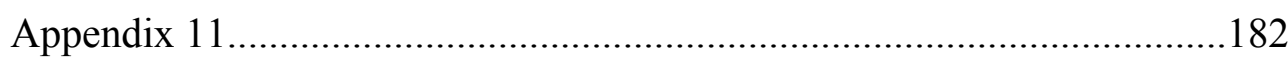




\section{List of Figures}

\section{Chapter 3.}

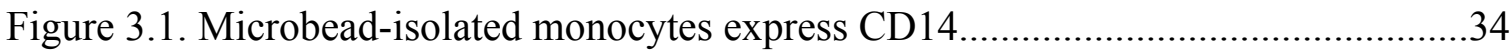

Figure 3.2. Microbead-isolated monocytes express CD40 upon LPS stimulation..............35

Figure 3.3. More than 95\% of Microbead-isolated cells express CD14...........................36

Figure 3.4. Work flow for monocyte and monocyte subset isolation................................37

Figure 3.5. Isolated monocyte subsets had above $95 \%$ purity............................................38

Figure 3.6. CD $14^{+}$monocytes express high levels of CD11b and CD14 and no CD16....39

Figure 3.7. Expression of CD11b, CD14 and CD16 on monocyte subsets.......................40

Figure 3.8. CD14 ${ }^{+}$monocytes express medium levels of CD11b, CD14 and CD16......40

Figure 3.9. Total monocytes from MS patients had high expression of activation markers.

Figure 3.10. $\mathrm{CD}_{14}^{+}$monocytes from MS patients had high expression of CD86, HLA-DR and CD64

Figure 3.11. CD16 ${ }^{+}$monocytes from MS patients had high expression of activation markers

Figure 3.12. Healthy CD14 ${ }^{+}$monocytes had higher expression of CD40 and CCR2 and lower expression of CD86 and HLA-DR, than the CD16 ${ }^{+}$monocytes.........46

Figure 3.13. MS CD16 ${ }^{+}$monocytes had higher expression of activation markers than $\mathrm{CD} 14^{+}$monocytes

\section{Chapter 4.}

Figure 4.1. Optimization of classical activation of monocytes....................................57

Figure 4.2. Optimization of GA treatment of monocytes..............................................58

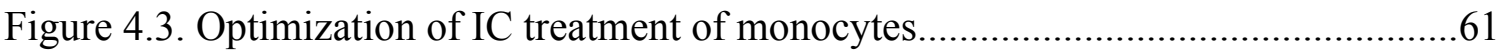

Figure 4.4. LPS stimulation induces classical activation of monocytes from healthy

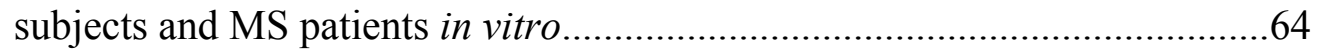

Figure 4.5. Type II activation of monocytes by GA and IC in vitro...............................6

Figure 4.6. Intracellular cytokine production after GA and IC treatments.......................71

Figure 4.7. qRT-PCR validation of results: mRNA expression of markers and cytokines in monocytes 


\section{Chapter 5.}

Figure 5.1. $\mathrm{CD} 14^{+}$monocytes were classically activated by LPS in both healthy and MS groups .84

Figure 5.2. $\mathrm{CD}^{+} 6^{+}$monocytes were classically activated by LPS in both healthy and MS groups .86

Figure 5.3. Healthy $\mathrm{CD} 14^{+}$and $\mathrm{CD} 16^{+}$monocytes were classically activated by LPS....88

Figure 5.4. MS CD14 $4^{+}$and $\mathrm{CD} 16^{+}$monocytes were classically activated by LPS...........90

Figure 5.5. Type II activation of $\mathrm{CD} 14^{+}$and $\mathrm{CD} 16^{+}$monocytes from healthy subjects...94

Figure 5.6. Type II activation of $\mathrm{CD}_{1} 4^{+}$and $\mathrm{CD} 16^{+}$monocytes from MS patients..........95

Figure 5.7. Classical activation of individually cultured and co-cultured CD14 ${ }^{+}$ monocytes from MS patients

Fihure 5.8. Classical activation of individually cultured and co-cultured CD16 ${ }^{+}$ monocytes from MS patients.

Figure 5.9. Type II activation of co-cultured subsets from healthy subjects

Figure 5.10. Type II activation of co-cultured subsets from MS patients 103

\section{Chapter 6.}

Figure 6.1. Proportion of $\mathrm{CD} 14^{+}$and $\mathrm{CD} 16^{+}$monocytes was altered in MS patients.....118

Figure 6.2. Type II activation of total monocytes by the in vivo GA treatment of MS patients......

Figure 6.3. Monocytes from GA-treated MS patients had reduced ability for classical activation in vitro.

Figure 6.4. In vitro GA and IC treatment enhances the in vivo type II activating effect of GA

Figure 6.5. HCA dendrogram and heat map of the three subject groups. 129

Figure 6.6. HCA dendrogram and heat map of in vitro LPS-stimulated monocytes......131

Figure 6.7. HCA dendrogram and heat map of in vitro GA-treated total monocytes.....132

Figure 6.8. HCA dendrogram and heat map of in vitro IC-treated total monocytes.......134 


\section{List of Tables}

\section{Chapter 5.}

Table 5.1. Type II activation of subsets by combination treatment in MS patients. .96

Table 5.2. Relative expression of activation markers after the combination treatment of $\mathrm{CD} 14^{+}$and $\mathrm{CD} 16^{+}$monocytes from MS patients......................104

Table 5.3. Type II activation of co-cultured subsets from MS patients. 104

\section{Chapter 6.}

Table 6.1. Proportion of $\mathrm{CD} 14^{+}$and $\mathrm{CD} 16^{+}$monocytes. 118

Table 6.2. Comparison of in vivo and in vitro GA effects on monocytes and their subsets in MS patients. 121

Table 6.3. HCA of in vitro LPS-stimulated total monocytes...... 131

Table 6.4. HCA of in vitro GA-treated total monocytes. 133

Table 6.5. HCA of in vitro IC-treated total monocytes. 134 


\section{Abbreviations}

AIDS - acquired immune deficiency syndrom

ANOVA - analysis of variance

APC - antigen presenting cells

$\mathrm{BBB}$ - blood brain barrier

$\mathrm{CBA}$ - cytometric bead array

CCR2 - C-C chemokine receptor 2 (or CD192)

CCL2 - C-C chemokine ligand 2

$\mathrm{CD}$ - cluster of differentiation

CD $14^{+}$monocytes - CD $14^{++} \mathrm{CD} 16^{-} \mathrm{CD} 11 \mathrm{~b}^{++}$human monocytes

$\mathrm{CD} 16^{+}$monocytes - $\mathrm{CD} 14^{+} \mathrm{CD} 16^{+} \mathrm{CD} 11 \mathrm{~b}^{+}$human monocytes

CNS - central nervous system

CTLA4 - cytotoxic T-lymphocyte antigen 4

DNA - deoxyribonucleic acid

ELISA - enzyme linked immunosorbent assay

ELISPOT - enzyme linked immunosorbent spot

$\mathrm{Fc} \gamma \mathrm{R}-\mathrm{Fc} \gamma$ receptor

$\mathrm{GA}$ - glatiramer acetate

$\mathrm{HCA}$ - hierarchial cluster analysis

healthy $\mathrm{CD} 14^{+}$monocytes $-\mathrm{CD} 14^{+}$monocytes from healthy subjects

healthy $\mathrm{CD} 16^{+}$monocytes $-\mathrm{CD} 16^{+}$monocytes from healthy subjects

HIV - human immunodeficiency virus

HLA-DR - human leucocyte antigen DR

IC - immune complex

Ig - immunoglobulin

IFN $\beta$ - interferon beta

IFN $\gamma$ - interferon gamma

IL - interleukin

IL-10R - interleukin 10 receptor

iNOS - inducible nitric oxide synthase

IVIG - intravenous immunoglobulin

LPS - lipopolysaccharide

MANOVA - multivariate analysis of variance

MFI - mean fluorescent intensity

MHC - major histocompatibility complex

mRNA - messenger ribonucleic acid

MBP - myelin basic protein

MOG - myelin oligodendrocyte protein

MS - multiple sclerosis

MS CD $14^{+}$monocytes $-\mathrm{CD} 14^{+}$monocytes from MS patients

MS CD $16^{+}$monocytes $-\mathrm{CD} 16^{+}$monocytes from MS patients

$\mu \mathrm{g} / \mathrm{ml}$ - microgram per milliliter

$\mu \mathrm{l}$ - microliter

$\mu \mathrm{M}$ - micromole

$\mathrm{ng} / \mathrm{ml}$ - monogram per milliliter

NK cells - natural killer cells

$\mathrm{NO}$ - nitric oxide 
OD - optical density

PBMC - peripheral blood mononuclear cells

$\mathrm{pg} / \mathrm{ml}$ - pictogram per milliliter

PLP - proteolipid protein

PPMS - primary progressive multiple sclerosis

qRT-PCR - quantitative reverse transcription polymerase chain reaction

$\mathrm{RBC}-$ red blood cells

RBC (A) - red blood cells from blood group A

$\mathrm{RBC}(\mathrm{O})$ - red blood cells from blood group $\mathrm{O}$

RNA - ribonucleic acid

RRMS - relapsing remitting multiple sclerosis

$\mathrm{SD}$ - standard deviation

SEM - standard error of the mean

SLAM - signaling leucocyte activation molecule

SPMS - secondary progressive multiple sclerosis

TGF $\beta$ - transforming growth factor beta

Th1 cells $-\mathrm{T}$ helper 1 cells

Th2 cells - T helper 2 cells

Th17 cells - T helper 17 cells

TLR4 - toll like receptor 4

$\mathrm{TNF} \alpha$ - tumor necrosis factor alpha

Treg - regulatory $\mathrm{T}$ cells

$\mathrm{U} / \mathrm{ml}$ - units per milliliter 
Chapter 1.

General introduction 


\subsection{Multiple sclerosis}

MS (MS) is an incurable disabilitating disease with unknown etiology, characterized by the loss of neuronal myelin sheath and subsequent neuron death. The autoimmune nature of MS is indicated by an involvement of immune cells in induction of inflammation and demyelinization of neurons in the CNS[1, 2], leading to cognitive, tactile, visual, hearing, speech and movement impairments[3]. Chronic CNS inflammation can persist for many years in MS patients and lead to significant disability and reduction in the quality of life[4].

MS mostly affects women[5] with an average onset at ages 20-40[6], although cases in younger individuals have been reported[7]. While MS is not a common disease, its prevalence is steadily increasing in the world with a highest rate in Caucasian subjects[8]. New Zealand is among the countries that have the highest incidence with 1 case reported in every $1400 \mathrm{New}$ Zealanders[9]. There are three main types of MS: relapsing remitting (RRMS), secondary progressive (SPMS) and primary progressive (PPMS). Up to $90 \%$ of cases begin as RRMS, which can progress to SPMS, and approximately $10 \%$ have PPMS[10]. The course of RRMS is defined by relapse and remission stages, where new brain lesions (regions of demyelinization) occur during the relapse stages with some to complete recovery during remissions[11]. In PPMS and SPMS, however, the remission stage is missing and the disease steadily progresses over years[12]. In all three forms of MS, immune dysregulation is the main component of the disease progression, although many environmental factors can influence MS outcome and progression.

The initiating cause of MS is unknown. Although multiple risk factors are associated with development of MS, including genetics, geographical distribution, vitamin D deficiency, smoking, diet and infections[13-18], none of these can be defined as a single causative factor. Instead, they are believed to be contributing factors that increase the likelihood of developing MS. Although the etiology of MS is still undefined, it is clear that pathological changes in the immune system play a main role in the development of the disease[19], reinforcing MS as an immune-mediated disease. 


\subsection{Immunology of MS}

Although the exact cause is unknown, MS is believed to be an immune mediated disease. Yet, immune cells play a dichotomous role in MS. While some immune cells, namely CD4 ${ }^{+}$ T helper cells (Th1 and Th17), natural killer, B cells, classically activated monocytes/macrophages and microglia cause inflammation in $\operatorname{MS}[1,2,20]$, immunoregulatory cells such as $\mathrm{T}$ helper 2 (Th2), regulatory $\mathrm{T}$ cells (Treg) and type II monocytes/macrophages protect the CNS from inflammation[21]. Th1 cells are a type of helper T lymphocytes that produce cytokines, such as IL-2 and IFN $\gamma$, and promote cellular immune defense against intracellular microorganisms[22]. The subset of Th cells, namely Th17, produces IL-17 cytokine and is involved in protection from extracellular microorganisms[23]. Differently from Th1 and Th17 cells, Th2 cells induce humoral immune response and produce cytokines, such as IL-4, IL-5, IL-6, IL-10 and IL-13[22]. Among these immune cells, inflammatory monocytes and T cells, such as Th1 and Th17 cells, are known as drivers of inflammation in MS[24]. Specifically, myelin-reactive Th1 and Th17 cells are known to be the main inflammatory cells that damage myelin sheaths of neurons in CNS, causing inflammation in $\operatorname{MS}[2,20,25]$.

Another class of $\mathrm{T}$ cells, such as $\mathrm{CD} 8^{+}$cytotoxic $\mathrm{T}$ cells has been also found in brain tissue of MS patients. The number of infiltrating $\mathrm{CD}^{+}$cells is significantly higher than that of $\mathrm{CD}^{+} \mathrm{T}$ cells in the CNS of MS patients and can be contributing to progression of the disease[26]. Other immune cells, such as B cells, are also involved in MS pathogenesis. Activated B cells have been found in cerebrospinal fluid of MS patients both in RRMS and SPMS[27]. While B cells are not able to migrate through the uninterrupted BBB, they are probably involved in late inflammation in MS[10].

During inflammation, T and B lymphocytes become activated by antigen presenting cells (APC). APC of monocytic lineage, such as microglia[28], dendritic cells[29] and monocytes[1] are found in increased number in the inflammatory tissue of MS patients. APC induce the activation of lymphocytes by presenting them antigens through MHCII molecules and co-stimulation through CD40 and CD86[30]. While Th1 and Th17 cell infiltration is evident in brain lesions in early stage of MS[20,31], monocytes are the predominant cells found in both early and late stages[32, 33], indicating the long term involvement of monocytes in inflammation during MS. These findings highlight the important role of monocytes in MS. 


\subsection{Monocytes}

\subsubsection{Monocytes as immune cells}

Monocytes are generated in the bone marrow from hematopoietic stem cells and mature in the peripheral blood. They have a half life of 1-3 days when circulating in the blood under normal conditions, and either infiltrate tissues to become tissue-specific macrophages and dendritic cells[34], or undergo apoptosis[35]. While in the blood, monocytes circulate in various stages of differentiation that define their function and characteristics[36], but can infiltrate tissues while in any of these stages[37].

Monocytes are an important cell type in the innate immune system and are a highly heterogeneous population of immune cells[36]. The heterogeneity of monocytes is not only due to their morphological characteristics, but also variability in their phenotype and activation[34]. In healthy subjects, monocytes make up approximately $10 \%$ of the total peripheral blood mononuclear cell population[38]. The number of monocytes can increase during some inflammatory and infectious diseases[35], either through replenishment from their less differentiated forms, or from their original source of generation[38].

\subsubsection{Characteristics of monocytes}

Monocytes can be distinguished by their typical morphological and phenotypical characteristics, although the latter can be highly variable. Morphologically, monocytes can be defined as round cells with a single nucleus and large cytoplasm, whereas their phenotype can be identified through a broad spectrum of cell surface markers uniquely expressed on different types of monocytes[35]. CD14 and CD11b are defined as a hallmark of monocytes and thus are expressed on all monocytes, but at different levels. CD14 is a receptor for the bacterial toxin, lipopolysaccharide (LPS), [39] and CD11b is a monocyte adhesion molecule and an integrin[40]. While these are markers that are systematically expressed on all monocytes, other markers, such as CD16 (Fc $\gamma$ receptor III), are found only in specific subsets of monocytes[41]. Classification of monocytes in relation to their phenotype is still a developing area of immunology. 


\subsubsection{Function of monocytes}

Despite the diversity in the characteristics of monocytes, all monocytes generally function in the defense and clearance of infectious or unnecessary elements. Thus, they act as scavenger cells and maintain homeostasis by removing toxic products and dead cells from the organism[38]. In addition, monocytes kill and eliminate pathogens through an internalization process, called phagocytosis[38], and through the production of toxic substances. In addition, monocytes give rise to other phagocytic cells, such as macrophages and dendritic cells[42]. Other immune cells that are implicated in MS, such as T cells, can be activated or suppressed by monocytes[21]. In addition to contributing to inflammation, monocytes can also regulate the immune response and control inflammation[43]. Besides their immune roles, it has been recently shown that monocytes facilitate tissue repair and wound healing[36]. Function of monocytes depend on their activation state[44] and they can be generally classified into four types: classically (M1), alternatively (M2a) and type II activated (M2b) and deactivated (M2c) monocytes[45]. M1 cells are classically activated monocytes that are responsible for killing and clearing microorganisms and inducing inflammation. M2a monocytes are alternatively activated monocytes, which are involved in defense against parasites and tissue repair. M2b monocytes are type II activated monocytes and are responsible for immunoregulation, whereas $\mathrm{M} 2 \mathrm{c}$ is a deactivated form of monocytes. Among them, classically and type II activated monocytes are known to be important in pathogenesis of MS[21]. Depending on their activation and on which cell surface markers and cytokines are elevated, monocytes induce either inflammatory Th1 and Th17 cells, or anti-inflammatory Th2 and Treg cell responses[46].

\subsubsection{Classical activation of monocytes}

When exposed to stimulants, such as interferon gamma (IFN- $\gamma$ ) and bacterial lipopolysaccharides (LPS), monocytes become classically activated and have characteristics that are typical of inflammatory cells. Classically activated monocytes can polarize $\mathrm{T}$ cells towards predominantly Th1 and Th17 types by producing high levels of inflammatory cytokines and molecules. As such, they are substantial producers of the cytokines IL-6 and IL-12[47, 48], and express high levels of CD40, CD86 and HLA-DR[49, 50], in comparison to inactive cells. A typical classical activation can be induced by in vitro stimulation of 
monocytes with IFN- $\gamma$ and LPS[51]. Therefore, IFN- $\gamma$-primed and LPS-stimulated monocytes can be used as an in vitro model of classical activation.

Monocytes are potent antigen presenting cells and can activate $\mathrm{T}$ cells during inflammation by binding of their co-stimulatory markers CD40, CD80 and CD86[52] to their ligands on $\mathrm{T}$ cells (CD40L and CD28 respectively) and present antigens through HLA molecules to T cell receptors (TCR). They also produce monokines, such as IL-1 $\beta$, IL-6, IL8, IL-10, IL-12 and TNF $[46]$ to induce activation of different T cells and other immune cells. Although the stimulating cause is unknown, classically activated monocytes activate T cells and induce inflammation in MS[53, 54].

\subsubsection{Type II activation of monocytes}

In contrast to classically activated M1 monocytes that drive a Th1 response, M2 monocytes produce high level of IL-10[55] and induce activation of Th2 cells[45]. Among the M2 monocytes, alternatively activated M2a monocytes produce substantial amount of growth factors and are responsible for tissue repair and angiogenesis[36], as well as inducing allergy[45]. While there is no evidence about an involvement of alternatively activated monocytes in MS, induction of type II activated M2b monocytes can be a therapeutic approach for the disease[21, 32].

Type II activated monocytes play an immunomodulatory role[21]. In vitro type II activation can be induced by treatment of monocytes with IL-10 and IL-13 in the presence of LPS[56]. These type II monocytes lead to activation of Th2 cells and, accordingly, are associated with amelioration of MS[32, 43]. Type II activated monocytes have a reduced production of inflammatory cytokines and lower expression of co-stimulatory markers and a higher level of the anti-inflammatory cytokine, IL-10, in comparison to classically activated monocytes. This enables inhibition of inflammatory Th1 and Th17 cell activation and shifting the $\mathrm{T}$ cell profile towards a Th2 response[21]. Ultimately, the polarization of $\mathrm{T}$ cells by monocytes can significantly affect the course of MS; for example, type II activated tissueresident macrophages prevented the onset of disease and ameliorate its progression in a mouse model of MS[44]. Thus, inhibition of classically activated monocytes by type II activation can be an advantageous method to ameliorate disease in MS. 


\subsubsection{Cell surface markers and cytokines in monocytes}

Activation of monocytes can be defined by the expression of cell surface markers, such as CD40, CD64, CD86, CCR2 and HLA-DR, and production of cytokines, including IL6, IL-10 and IL-12. CD40 is a receptor of TNF family, expressed on many immune cells, including monocytes. Binding of CD40 to its ligand (CD40L) co-stimulates Th1 and Th17 cells and contributes to progression of autoimmune diseases[57]. CD86 is another costimulatory marker for T cells. Whereas binding of CD86 to its ligand CD28 activates T cells, the binding to CTLA-4 inhibits T cell activation[58]. CD64 (Fc $\gamma \mathrm{RI})$ is a high affinity receptor for IC and Ig (immunoglobulins). Its role is to mediate phagocytosis and defense from microorganisms[59]. CCR2 is a chemokine receptor for CCL2 chemokine which binding facilitates migration of monocytes to inflammatory site[60]. HLA-DR is an antigenpresenting molecule of MHC class II. Presentation of antigens through HLA-DR induces proliferation of T cells[61]. IL-6, or interleukin 6, promotes differentiation of Th17 cells and downregulates activation of mature Th17 cells[62, 63]. IL-10 is an anti-inflammatory cytokine that plays an immunomodulatory role. It is involved in polarization of $\mathrm{T}$ cell response by inhibiting proliferation and activation of Th1 and Th17 cells[64]. IL-12 plays the main role in promoting Th1 cell response and inducing inflammation[65]. Classically activated monocytes have increased levels of CD40, CD86, CD64, CCR2, HLA-DR, IL-6 and IL-12, and these molecules are upregulated during multiple sclerosis[66-68]. In contrast, type II activated monocytes are characterized by having lower levels of these markers and cytokines and increased production of IL-10[21].

\subsubsection{Role of monocytes in inflammation and MS}

Monocytes play an inflammatory role when they encounter microorganisms, but can also induce inflammation in a non-infectious environment. During inflammation, circulating or tissue resident monocytes activate $\mathrm{T}$ cells by producing inflammatory cytokines, such as IL-1, IL-6, IL-12 and TNF- $\alpha$, and attracting them to the inflammatory site[69, 70]. Monocytes bind attracted T cells through their co-stimulatory molecules CD40[71] and CD86[52] and present antigens bound to HLA-DR[24, 31] molecules.

During MS, monocytes damage the nerve cells in the absence of overt infection[15]. It has been shown that monocytes can infiltrate the CNS and become important mediators of 
inflammation in a mouse model of MS[72]. The inflammatory products of monocytes, including IL-6, IL-12, CD40, CD86 and HLA-DR, are significantly upregulated during MS and are believed to be a key mechanism through which inflammatory damage occurs in the brain tissue[47-50]. However, the role of inflammatory monocytes is not restricted to their activation of other immune cells. In addition to activating Th1 and Th17 cells, monocytes can directly damage neurons in MS[73]. They are one of the first cell types that arrive in the brain tissue to drive inflammation in MS, and are the most permanent cells that exist in all the active lesions of MS patients[24]. Intralesional monocytes have been shown to phagocytose debris derived from the neuronal myelin sheath[24], indicating their direct involvement in the nerve damage. This direct involvement has been shown by the observation that monocytes can lyse nerve cells by producing neurotoxic factors, such as nitric oxide and oxygen radicals[74, 75]. Due to their high production of migration factors such as matrix metalloproteinases, monocytes are more effective at migrating through the blood brain barrier (BBB) and infiltrating the brain tissue than $\mathrm{T}$ cells[76]. Together, the monocyte-derived cytokines and activation molecules stimulate the inflammatory Th1 and Th17 cells to induce inflammation and neuronal death in MS[24]. These findings emphasize the crucial role of monocytes in MS, reinforcing monocytes as an attractive target for the treatment of MS. 


\subsection{Monocyte subsets}

\subsection{1 $\mathrm{CD}^{+} 4^{+}$and $\mathrm{CD} 16^{+}$monocyte subsets}

There are two main subsets of monocytes that are identified as $\mathrm{CD} 14^{++} \mathrm{CD} 16^{-}$(i.e. $\mathrm{CD} 14^{+}$ monocytes) and $\mathrm{CD} 14^{+} \mathrm{CD} 16^{+}$(i.e. $\mathrm{CD} 16^{+}$monocytes). In healthy subjects, $90-95 \%$ of monocytes are $\mathrm{CD}_{14}{ }^{+}$and the other $5-10 \%$ are $\mathrm{CD} 16^{+}$. Monocytes originate from hematopoetic stem cells in the bone marrow and enter the blood stream as CD14 $4^{+}$monocytes, where they either continue to circulate as $\mathrm{CD} 14^{+}$monocytes, or differentiate into $\mathrm{CD} 16^{+}$ monocytes[77]. The $\mathrm{CD} 16^{+}$monocytes are believed to give rise to tissue macrophages and dendritic cells[78]. The two monocyte subsets have functional and phenotypical differences. For example, CD14 ${ }^{+}$monocytes express higher levels of CCR2[41, 79], CD64[80] and IL$10[81]$; whereas, CD16 ${ }^{+}$monocytes express higher levels of CD40, CD86 and HLA-DR[80, $82]$ and inflammatory cytokines[79, 83, 84].

Although both subsets bear typical morphological characteristics of monocytes[85], they differ in phenotype and function. These variations are defined by the differential expression of markers, production of cytokines and antigen presenting abilities of $\mathrm{CD}_{1} 4^{+}$and $\mathrm{CD} 6^{+}$monocyte populations. Thus, in comparison to $\mathrm{CD}_{1} 6^{+}$subset, CD $14^{+}$monocytes express higher level of chemokine receptor CCR2[41, 79] and Fc $\gamma$ receptor I (CD64)[80], while producing higher level of anti-inflammatory cytokine IL-10[81]. In contrast to CD14 monocytes, $\mathrm{CD}_{16}{ }^{+}$monocytes have higher level of chemokine receptor CX3CR1[41], markers HLA-DR, CD40 and CD86[80, 82] and inflammatory cytokines TNF $\alpha$, IL-6 and IL$1 \beta[79,83,84]$, when exposed to bacterial or viral antigens. Not surprisingly, CD16 ${ }^{+}$ monocytes are far superior at activating $\mathrm{T}$ cells[82], suggesting that they are more active inducers of inflammation than the $\mathrm{CD} 14^{+}$monocytes.

\subsection{2 $\mathrm{CD}^{+} 4^{+}$and $\mathrm{CD} 16^{+}$monocytes in inflammation and MS}

The ratio of the two monocyte subsets is altered during inflammatory and autoimmune diseases[86-90], and an increased number of $\mathrm{CD}^{+} 6^{+}$monocytes has been shown to be correlated with expansion of Th1 cells[91] and activation of inflammation[87]. While the absolute number of the total monocyte population remains normal, the percentage of CD16 ${ }^{+}$ monocytes within the total monocyte population increases during infections[92, 93]. 
Expansion of the $\mathrm{CD}^{+} 6^{+}$monocytes is observed in inflammatory diseases such as rheumatoid arthritis[86], lupus erythromatosus[87], atherosclerosis[88], Kawasaki disease[94], immune thrombocytopenia[90] and MS[95]. This increase is positively correlated with an activation of inflammatory processes[96], high levels of C-reactive protein (CRP), a marker of inflammation, [94], and proliferation of Th1 cells[90]. Moreover, a recent in vitro study revealed that the expanded $\mathrm{CD}_{1} 6^{+}$monocyte subset, and not the $\mathrm{CD} 14^{+}$subset, is directly responsible for the expansion of Th1 cells and inhibition of regulatory $\mathrm{T}$ cells in immune thrombocytopenia[90]. This research highlights that the change in the ratio of the monocyte subsets reflects their unique role in inflammation and infection.

Similar to other inflammatory diseases, although the total monocyte count is generally normal in patients with MS, the percentage of CD14 $4^{+}$and $\mathrm{CD} 16^{+}$monocyte subsets within the total monocyte population changes dramatically[95]. The proportion of circulating CD14 ${ }^{+}$ monocytes is reduced from $90-95 \%$ in healthy subjects to $70 \%$ in MS patients. On the other hand, the proportion of $\mathrm{CD}_{1} 6^{+}$monocytes is significantly higher in MS patients (32\%), than in healthy subjects, which corresponds to a 3-6 fold increase from the normal range (510\%)[97]. Lopez-Moratalla et al. suggests that $\mathrm{CD}_{1} 4^{+}$monocytes give rise to $\mathrm{CD} 16^{+}$ monocytes[97]. Therefore, the change in ratio of $\mathrm{CD} 14^{+}$and $\mathrm{CD} 16^{+}$monocytes might be due to differentiation of some of the $\mathrm{CD}_{1} 4^{+}$subset monocytes into $\mathrm{CD}_{1} 6^{+}$cells. Interestingly, the decreased number of $\mathrm{CD} 14^{+}$monocytes is negatively correlated with the Th1/Th2 cell ratio in MS[98], suggesting that $\mathrm{CD} 14^{+}$monocytes are not responsible for the significant expansion of inflammatory Th1 cells. It is not clear whether the proliferation of these $\mathrm{T}$ cells is related to the expansion of $\mathrm{CD} 16^{+}$monocytes in MS. Understanding the specific roles of $\mathrm{CD} 14^{+}$and $\mathrm{CD}_{16}{ }^{+}$monocytes in MS is crucial; however, currently the function and phenotype of the individual $\mathrm{CD} 14^{+}$and $\mathrm{CD} 16^{+}$subsets in MS, and their exact role in MS and the expansion of inflammatory T cells, is still unknown.

\subsubsection{Activation of CD14 ${ }^{+}$and $\mathrm{CD} 16^{+}$monocytes}

Monocytes in the circulation function as inflammatory cells during MS[97] and it is known that both $\mathrm{CD} 14^{+}$and $\mathrm{CD} 16^{+}$monocytes have pro-inflammatory roles when classically activated in inflammatory conditions[79]. As both subsets express TLR4, an LPS receptor[99], in vitro treatment of monocytes with LPS can classically activate both subsets and induce a pro-inflammatory phenotype. Both types of monocytes are induced to produce inflammatory cytokines, such as IL-6 and TNF- $\alpha$, by LPS stimulation[81]; however, the main 
producers of TNF- $\alpha$ are not $\mathrm{CD} 14^{+}$monocytes, but $\mathrm{CD}_{1} 6^{+}$monocytes[83]. Expansion of $\mathrm{CD}^{+} 6^{+}$monocytes in Kawasaki disease is associated with a high level of CRP[94], a biomarker of systemic inflammation, suggesting an association of $\mathrm{CD}^{+} 6^{+}$monocytes with inflammation. An increased number of $\mathrm{CD}^{+} 6^{+}$monocytes has been correlated with high levels of Th1 cells in immune thrombocytopenia, and $\mathrm{CD}_{1} 6^{+}$monocytes are also directly responsible for extensive production of the inflammatory cytokine IL-12 (higher than in $\mathrm{CD} 14^{+}$monocytes) and increased proliferation of IFN $-\gamma^{+}$Th1 cells in vitro[90]. This suggests that classically activated $\mathrm{CD}^{+} 6^{+}$monocytes are potent activators of Th1 cells during inflammation and might be more efficient at inducing inflammation than $\mathrm{CD} 14^{+}$monocytes.

Although monocytes in MS patients appear to have characteristics of classically activated cells[97], the phenotypes of these classically activated individual CD14 ${ }^{+}$and CD16 $6^{+}$ subsets have not yet been described. In contrast to classically activated monocytes, type II activated monocytes exhibit features of anti-inflammatory or regulatory immune cells, and can be beneficial in treatment of MS[21]; however, to date, there have been no studies characterizing type II activation of $\mathrm{CD} 14^{+}$and $\mathrm{CD} 16^{+}$monocyte subsets in MS. Very little is known about the regulatory effect of immunomodulatory agents on monocyte subsets. Drugs such as glucocorticoids induce regulatory type of macrophages[36] and have an immunosuppressive effect on both monocyte subsets. Glucocorticoid treatment leads to a reduction in HLA-DR expression and the number of $\mathrm{CD}_{16}{ }^{+}$monocytes in the circulation of both MS patients and healthy subjects, whereas the number of $\mathrm{CD} 14^{+}$monocytes is increased[95, 100]. The well-known immunosuppressive effect of glucocorticoids in MS is not through inhibition of $\mathrm{CD}_{1} 4^{+}$monocytes and other immune cells, but mainly through depletion of $\mathrm{CD}_{16} 6^{+}$monocytes[95], showing the importance of $\mathrm{CD}_{1} 6^{+}$monocytes as a target for the treatment of MS. Although $\mathrm{CD}_{1} 6^{+}$monocytes are only a minority of the monocytes in healthy human blood[77], the role of $\mathrm{CD} 16^{+}$monocytes in MS, as well as $\mathrm{CD} 14^{+}$monocytes, should be well considered.

\subsubsection{Role of $\mathrm{CD} 14^{+}$and $\mathrm{CD} 16^{+}$monocytes in brain inflammation and MS}

Although both monocyte subsets can be involved in brain inflammation, $\mathrm{CD}_{16}^{+}$ monocytes have been shown to be important cells in the progression of AIDS-related encephalitis, and are more effective at invading the CNS than CD14 ${ }^{+}$monocytes[101]. Despite the fact that $\mathrm{CD}_{1} 6^{+}$monocytes are released from the bone marrow into the circulation later than $\mathrm{CD} 14^{+}$monocytes, they spend less time in the blood and migrate into 
tissues earlier than $\mathrm{CD}^{+} 4^{+}$monocytes in a macaque model of AIDS[102]. After transmigration into the $\mathrm{CNS}, \mathrm{CD} 16^{+}$monocytes surround blood vessels and differentiate into macrophages[103], which cause neuron death in HIV dementia[104]. Taken together, these studies suggest that $\mathrm{CD}_{1} 6^{+}$monocytes can effectively infiltrate the brain, take the form of inflammatory macrophages and cause nerve damage, suggesting an importance of differentiating between monocyte subsets in relation to their function in brain inflammation.

Although a significant change in the proportion of $\mathrm{CD}^{+} 4^{+}$and $\mathrm{CD} 16^{+}$monocytes has been found in MS[95], there are no studies that have looked at the specific phenotypes and functions of these $\mathrm{CD} 14^{+}$and $\mathrm{CD}_{1} 6^{+}$subsets so far. However, in other autoimmune diseases, an increased number of $\mathrm{CD}^{+} 6^{+}$monocytes correlates with expansion of Th1 cells[90] and induction of inflammation[105]. During MS, monocytes play an important role in inducing Th1 and Th17 cells[24], and damaging nerve cells[73]; however, it is unclear what roles the $\mathrm{CD}_{14}{ }^{+}$and $\mathrm{CD}_{1} 6^{+}$monocyte subsets play in this inflammation, and how they can be altered by immunomodulating treatments for MS. 


\subsection{Treatments of MS}

Most MS treatments target the immune system, such as glatiramer acetate (discussed in section 1.6), interferon (IFN)- $\beta$, mitoxantrone[106], fingolimod[107], natalizumab[108], glucocorticoids[109], Teriflunomide[110] and BG-12[111]. All of these treatments are approved by the food and drug administration (FDA) as treatments for MS, and are mostly for use in RRMS patients. Although these drugs have 30-70\% efficacy in reducing relapse rates and disability, none of them are considered a cure for MS[112, 113] and are known to have significant side effects. Other, non-FDA approved drugs such as campath[114] have also been efficient in some cases of MS but, likewise, can cause many side effects.

\subsubsection{IFN- $\beta$}

Currently, there are four IFN- $\beta$ products available on the market: Betaferon, Avonex, Extavia and Rebif. IFN- $\beta$ is an immunomodulating agent which inhibits Th1 cell activation[115] and enhances anti-inflammatory IL-10 production by Th2 cells. Despite that, it is believed that IFN- $\beta$ works through other immune cells and does not have a direct effect on $\mathrm{CD}^{+}$or $\mathrm{CD}^{+} \mathrm{T}$ cells[116]. In addition, it has been shown that IFN- $\beta$ can directly modulate B cells and cells of monocytic lineage, such as macrophages and microglia[115, 117]. Thus, IFN- $\beta$ inhibits antigen presentation by macrophages and $B$ cells to Th1 cells[115], as well as activation of microglial cells[117]. However, the exact mechanism of its action is unknown. A disadvantage of this treatment is that in some cases the effectiveness of IFN- $\beta$ may be reduced as a result of the development of anti-IFN- $\beta$ antibodies[118]. Despite the widespread use of IFN- $\beta$ for treatment of MS, this disadvantage, along with other potential side effects, such as flu-like symptoms[119], has led to a decrease in the popularity of IFN- $\beta$ therapy.

\subsubsection{Mitoxantrone (Novantrone)}

Mitoxantrone is an antineoplastic chemotherapy agent that has some effect in reducing relapses and progression of MS, and is the only FDA-approved drug for both RRMS and SPMS[120]. Its effect on the immune system is related to its cytotoxicity to immune cells[121]; however, this extensive cell death can result in leukopenia[122], along with other 
side effects such as nausea, vomiting, alopecia and amenorrhoea, and improvements in the disability score have not been significant in MS patients[123]. Moreover, due to a significant cardiotoxicity, the dose of the drug is limited to a lifetime cumulative dose[124].

\subsubsection{Fingolimod (Gilenya)}

Fingolimod is a new oral immunotherapy[125] which inhibits migration of inflammatory Th1 and Th17 cells into the CNS by regulating its migration-related receptor sphingosine 1phosphate[126]. Fingolimod reduces number of brain lesions and relapses in MS patients with RRMS, and has been more effective than IFN- $\beta$ treatment[127]. However, death cases associated with reactivation of latent viral infections after the administration of this drug have been reported in MS patients[127].

\subsubsection{Natalizumab (Tysabri)}

Natalizumab is another inhibitor of $\mathrm{T}$ cell migration that works by blocking the $\mathrm{T}$ cell adhesion molecule $\alpha 4 \beta 1$-integrin. It was recently removed from the list of FDA approved drugs due to cases of drug-related death[108] and then re-approved as an efficient treatment for MS, but natalizumab treated MS patients require close monitoring for adverse effects of the treatment, such as progressive multifocal leukoencephalopathy, or PML[128, 129].

\subsubsection{Glucocorticoids}

Glucocorticoids (GC) are the synthetic form of endogenous steroid hormones and have an immunosuppressive effect in MS[130]. Despite the fact that GC are not in the list of FDAapproved treatments for MS, they have been a commonly used modality for the treatment of MS[131] and have a broad effect on the immune system[132]. Although GC have a wide range of inhibitory effects on the immune cells, including suppression of their migration through the blood brain barrier (BBB), decreased activation, and increased cell death[133137], they only have short term, partial efficacy with respect to improving relapse rates after administration of high doses[131]. As GC treatment does not have a long lasting effect on MS and involves significant systemic side effects including, but not limited to, depression, osteoporosis, diabetes and infections[138], GC treatment is appropriate only in drug-resistant cases, but not as a first choice therapy for MS. 


\subsubsection{Alemtuzumab (Campath-1H)}

Alemtuzumab is a monoclonal anti-CD52 (a lymphocyte anti-adhesion molecule) antibody that suppresses inflammation through depletion of circulating $\mathrm{T}$ and $\mathrm{B}$ cells[139] and prevents development of new brain lesions in MS[140]. Initially being developed as a leukemia treatment, alemtuzumab has shown to be effective in reducing relapses in RRMS only, but not any other types of MS[141, 142]. Although an administration of alemtuzumab is required only once every six months, it only affects newly formed brain lesions, whereas previously formed brain inflammation and progressing disability have not been improved by treatment with alemtuzumab in MS patients[140]. As it is typical for immune-depleting agents, alemtuzumab has the potential to cause leukopenia which increases susceptibility to infections[143].

\subsubsection{IVIG (intravenous immunoglobulin G)}

IVIG is concentrated natural immunoglobulin $\mathrm{G}$, derived from the blood of hundreds of healthy donors and is administered once a month in a dose of $0,4-2 \mathrm{~g} / \mathrm{kg}[144]$. The cost of IVIG treatment for an average $60 \mathrm{~kg}$ person is about 120 New Zealand dollars per month. IVIG is well known for its minimal and rare side effects, which include anemia, hypersensitivity, headache, fever, and, in number of cases, renal toxicity[145]. At present, only a few studies have been done to explore its mechanism of action on the immune system. IVIG injection results in a decrease in number of $\mathrm{CD} 16^{+}$, but not $\mathrm{CD} 14^{+}$, monocytes in Kawasaki disease[94]. In human MS studies, IVIG has some effectiveness in reducing relapse rates and the clinical symptoms of the disease[146-149]. A study by Bayry et al, found that in human monocyte-derived dendritic cells, IVIG down-regulates production of pro-inflammatory cytokines[150], including IL-12 production in MS patients[151], and expression of MHC II molecules and the co-stimulatory markers CD40 and CD86 in vitro. These findings demonstrated that IVIG has a type II activating effect on dendritic cells. Although it has been established that IVIG has minimal side effects[147], its mechanism of action in MS is unclear. 


\subsubsection{Teriflunomide}

Teriflunomide is an oral disease-modifying drug. It modulates immune system through inhibition of dihydro-orotate dehydrogenase and pyrimidine synthesis[152]. Teraflunomide reduces number of annual relapses in RRMS patients. Although it has been considered as a safe therapy for MS in short-term studies, more studies are required in order to define its long-term effect[110].

\subsubsection{BG-12 (Tecfidera)}

BG-12, or dimethylfumarate, is an oral neuroprotective agent and protects nerve axons from oxidative damage[153]. It has been approved by FDA as an MS treatment in 2013. BG12 is known to be effective in decreasing relapse rates and disability in patients with RRMS[154]. However, the effect of BG-12 on immune system is not clear.

\subsubsection{Summary}

None of the current treatment options are curative for MS and overall the benefits for the patients do not always balance against their side effects. Moreover, they are extremely costly and need to be administered in the long term[155]. As the modest efficacy of the treatments can be associated with extensive side effects, a new approach for the treatment of MS is required. A difficulty with finding a highly effective treatment is the unknown etiology and complex, yet unclear, mechanism of the disease progression. While the mechanism of MS development needs to be clarified, it is essential to continue to explore safe and effective alternative treatment modalities. Therefore, in this study, we investigated treatments that target monocytes such as glatiramer acetate (GA) and immune complexes (IC) in MS. 


\subsection{Glatiramer acetate}

\subsubsection{Glatiramer acetate in MS}

Glatiramer acetate (GA) is a current therapy for MS and is a random copolymer of four amino acids. GA has approximately 30\% efficacy in reducing relapses and disability in MS patients[156]. Aside from inflammation at the injection site due to daily subcutaneous injections, GA is known as a treatment with minimal, self-limiting side effects which, in rare cases, can involve flushing, chest tightness, palpitation, anxiety, and dyspnea[156]. One of the reasons for the limited efficacy of GA was the insufficient knowledge of its mechanism of action in MS, and thus, an understanding of which patient populations will best benefit from it.

The mechanism of GA action is not fully understood, although it has long been thought that the main effect of GA on MS is mediated by T cells, as GA has a direct inhibitory effect on Th1 cells and polarizes the $\mathrm{T}$ cell profile from a Th1 to Th2 cell direction[157]. This polarization changes the $\mathrm{T}$ cell-derived cytokine cascade[158]. It has been shown that GA increases the anti-inflammatory cytokines IL-10, IL-4 and TGF- $\beta$ and decreases proinflammatory TNF- $\alpha$ mRNA levels in T lymphocytes[159]. As an antigen, it pushes GAspecific Th2 cells to induce anti-GA antibody production by B cells[160]. These anti-GA antibodies are found at higher levels than anti-MBP antibodies in MS patients treated with GA[161]. GA acts on T cells as an "antigen mimic" and blocks the inflammatory Th1 cell activation through suppressing their response to nerve antigens such as MBP, PLP[162] and MOG[163, 164]. These antigens are presented to $\mathrm{T}$ cells by antigen presenting cells, including monocytes, through HLA-DR molecules. GA-specific T cells have been shown to be abundant in MS lesions only in earlier stages, yet GA has long term efficacy in MS[165]. Since GA is also effective in late MS, when T cell infiltration is not prominent in the CNS, T cells must not be the only mediators of GA action[20].

It has been recently found that GA can act through an HLA-DR independent mechanism[166], suggesting that the antigen blocking on $\mathrm{T}$ cells is not necessarily a requirement for GA to be effective. This finding has led to further research to determine whether there are other regulatory cells that mediate the GA effect on T cells. It has been suggested that GA affects $\mathrm{T}$ cells in the periphery, with a subsequent migration of GAspecific Th2 cells to the CNS, where they inhibit the inflammatory Th1 cell activation[167, 
168]. In the peripheral blood, GA can encounter other immune cells, including regulatory $\mathrm{T}$ cells (Treg) - one of the major regulators of T cell activation. However, involvement of Treg in mediating the GA effect is unlikely, as Toker et al. have shown that an inhibition of Th1 cells by GA occurs independently from Treg in a mouse model of MS[166]. Therefore, there must be other regulators that can mediate the full GA effect on the immune system in MS. Recently, it has been shown that GA modulates activation of differentiated and tissue resident form of monocytes, such as microglia. Thus, GA increased the secretion of IL-10, while decreasing the production of TNF $\alpha$ in rat microglia[169]. These findings indicate on the ability of GA to modulate cells of monocytic lineage.

\subsubsection{Effect of glatiramer acetate on monocytes and their subsets}

Human monocytes are the most abundant circulating antigen presenting cells that modulate $\mathrm{T}$ cell activation in the periphery. More than $95 \%$ of murine peripheral monocytes specifically bind GA in vivo 3-6 hours after GA administration. Recently, it has been shown that GA can directly affect an intermediate $\mathrm{CD} 14^{++} \mathrm{CD} 16^{+}$subset of monocytes and increase its phagocytic activity in MS patients[170]. GA has a direct type II activating effect on human monocytes, resulting in a regulatory phenotype[32]. This is consistent with the finding that in a mouse model of MS, type II activated GA-treated monocytes can directly inhibit Th1 cell proliferation[166]. Moreover, GA-treated monocytes ameliorate MS in mice, by inducing their regulatory phenotype[43]. All the above-mentioned studies lead to a hypothesis that the main mediators of GA's effect in MS are the circulating blood monocytes. In order to fully understand the mechanism of action of GA and improve its efficacy, it is crucial to elucidate the GA effect on human monocytes.

Although GA has an immunomodulatory effect on human monocytes in MS[32], its effect on specific monocyte sub-populations has not been described yet. To date, there is no evidence about type II activation of $\mathrm{CD} 14^{+}$and $\mathrm{CD} 16^{+}$monocyte subsets in MS, despite the fact that these monocyte subsets play unique roles in inflammation[82, 83, 94]. Given that exploring the mechanism of action of GA on monocytes is essential to improving treatment efficacy, this study looks at the effect of GA on monocytes, and the individual $\mathrm{CD}_{14}^{+}$and $\mathrm{CD}^{+} 6^{+}$subsets, in MS. 


\subsection{Immune complex}

Immune complexes (IC) consist of antigens bound to antibodies. Although IC form naturally due to binding of human antibodies to pathogens as part of a defense mechanism, other IC form as a result of antibodies that bind to various human cells and molecules. While these complexes can be pathogenic, as seen in lupus erythematosus[171] and rheumatoid arthritis[172], IC can also induce type II activation of macrophages and thus, reduce inflammation.

Recently it has been shown that IC treatment switches the balance of cytokine production to the anti-inflammatory side in differentiated monocytes (macrophages) from mice [173], indicating a direct anti-inflammatory effect of IC on monocytes. More exploratory studies have been done using a mouse model of MS and have shown that IC improve the disease course by type II activating a differentiated form of monocytes (macrophages) and inhibiting expression of $\mathrm{CD} 40, \mathrm{CD} 80$, and IL-12, while upregulating anti-inflammatory IL-10 production[44]. A direct type II activating effect of IC has also been shown in murine macrophages in vitro, in which IC treatment in the presence of LPS downregulates the production of IL-12 and enhances the production of IL-10[173]. Although the type II activating effect of IC on these mature monocytes is well established in mice, little is known about the effect of IC on human monocytes.

Murine studies use IC of sheep red blood cells (SRBC) and anti-SRBC antibodies, whereas in humans the IC consists of human RBC and human IgG. In humans, IC can be generated naturally by intravenous immunoglobulin G injection (IVIG), which results in IC consisting of IVIG bound to the A and B antigens on human red blood cells[174-176]. These IVIG complexes have been shown to be an effective treatment for many autoimmune diseases[177-179]. In vitro studies have found that IC effectively bind to Fcy receptors on human monocytes[178, 180], therefore in vitro treatment of monocytes with IC gives a good representation of the biological effect of IVIG treatment in humans[176]. In addition, by exploring the effect of IC on monocytes, we may be able to explain the immunomodulatory effect of IVIG on monocytes. Despite the evidence regarding the regulatory effect of IVIG treatment in MS, the direct mechanism of action of IC on human monocytes and their subsets in MS is not yet clear. 


\subsection{Overall aims}

This study aims to investigate a type II activating effect of GA and IC on human monocytes in both healthy subjects and MS patients. In order to assess this, the following specific aims will be addressed:

1. To characterize the monocytes and their subsets in healthy and MS groups.

2. To compare the classical activation of monocytes and their subsets in healthy and MS groups.

3. To investigate type II activation of monocytes and their subsets by GA and IC 
Chapter 2.

Materials and methods 


\subsection{Subjects and blood samples}

Healthy volunteers were recruited from Victoria University of Wellington, and MS patients were recruited from the Wellington Hospital, Neurological Foundation of New Zealand and Wellington MS Society. Experimental protocols were approved by the Multiregion Ethics Committee and the Central Regional Ethics Committee (Ministry of Health, New Zealand) under the licences MEC 10/05/048 and CEN 11/11/062. All the volunteers gave informed written consent to participate in this study.

The healthy group included 30 healthy volunteers with a mean age of $35( \pm 13)$ years and a female to male ratio of 1.7:1. Healthy subjects did not have any underlying diseases and subjects who had any inflammatory or infectious diseases, including the common cold, or who had taken any medications, were excluded from the study. Female subjects were not pregnant or taking any contraceptives.

The MS patients group included 27 volunteers, each with a confirmed diagnosis of MS. 7 MS patients were under daily glatiramer acetate treatment (GA-treated MS group) and $20 \mathrm{MS}$ patients had received no treatments for a minimum of 6 months (untreated or non-GA treated MS group). Patients who were receiving treatments other than GA were not included in the study. The MS patients had either relapsing remitting MS (RRMS) and were in a relapse stage at the time of study participation, secondary progressive MS (SPMS), or primary progressive MS (PPMS). The mean age of the patients was $47( \pm 12)$ years and there was a female to male ratio of $2: 1$. The disease severity was evaluated by qualified neurologists according to a commonly used scoring system known as the expanded disability status scale (EDSS). The mean EDSS of patients in this study was $4( \pm 2)$, where an EDSS of 1 represents the minimum severity of MS, and 10 represents the highest severity or death due to MS (Appendix 1)[181]. The mean duration of MS was $10( \pm 9)$ years.

Up to $50 \mathrm{ml}$ of peripheral venous blood was taken from healthy subjects, and up to 100 $\mathrm{ml}$ of blood from the MS patients. The blood collection (phlebotomy) was performed either at the Department of Neurology at Wellington Hospital, or in the phlebotomy room at the Malaghan Institute of Medical Research, VUW, by a certified phlebotomist. The blood samples were collected into sterile heparinised vaccutainer tubes (BD Biosciences, San Jose, CA, USA). All necessary precautions were taken to minimise the potential side effects from the phlebotomy. The blood samples were used for isolation of peripheral blood mononuclear cells (PBMC) and subsequent experiments immediately after collection. 


\subsection{Total monocyte isolation}

\subsubsection{PBMC isolation}

To $50 \mathrm{ml}$ of heparinised whole blood, $93.3 \mathrm{ml}$ of phosphate buffered saline (PBS) without calcium or magnesium was added, and the sample mixed by inversion. $50 \mathrm{ml}$ of Histopaque-1077 (Sigma, St. Louis, MO, USA) was brought to room temperature. The bloodsaline mixture was layered onto the Histopaque-1077 and centrifuged at $400 \mathrm{x} \mathrm{g}$ for 30 minutes at room temperature. After centrifugation, the upper layer was aspirated and the opaque interface containing the PBMC was collected. The PBMC were washed with PBS and centrifuged at $250 \mathrm{x}$ g for 10 minutes, two times. Finally, the PBMC were washed with MACS isolation buffer to prepare them for monocyte isolation.

\subsubsection{Isolation of total monocytes from PBMC}

The monocytes were magnetically labelled according the following protocol, to facilitate isolation. The PBMC were resuspended in $80 \mu 1$ of MACS buffer per $10^{7}$ cells, and incubated with human CD14 microbeads (Myltenyi Biotec, Teterow, Germany) at a concentration of 20 $\mu 1$ per $10^{7}$ cells. After incubation at $4^{\circ} \mathrm{C}$ for 15 minutes, the cells were washed in $1-2 \mathrm{ml}$ of MACS isolation buffer and centrifuged at $300 \mathrm{x}$ g for 10 minutes. The cells were resuspended in MACS isolation buffer, ready for magnetic separation.

The magnetic separation of the total monocyte population from the other PBMC was performed using LS columns on a magnet (Myltenyi Biotec, Germany). The column was washed with MACS isolation buffer, then the magnetically labelled cells were added to the column, and the unlabeled CD14 cells passed through the column. The column was washed 3 times with $3 \mathrm{ml}$ of MACS isolation buffer, while still on the magnet. The column with the remaining $\mathrm{CD} 14^{+}$cells was removed from the magnet and placed on a collection tube. The $\mathrm{CD} 14^{+}$cells (monocytes) were then flushed through to the collection tube and used for cell culture. 


\subsection{Isolation of subsets}

\subsubsection{Depletion of NK cells and granulocytes}

The PBMC were isolated as described above (Section 2.3.1) and magnetically labelled with CD15 and CD56 microbeads (Miltenyi Biotec, Germany), specific for granulocytes and natural killer cells, respectively. The labelled granulocytes and natural killer cells were magnetically separated on LD columns as described previously (Section 2.3.2), and the effluent containing the monocytes and lymphocytes was taken for the isolation of $\mathrm{CD} 16^{+}$ monocytes.

\subsubsection{Positive isolation of $\mathrm{CD} 16^{+}$monocytes}

The collected effluent was magnetically labelled with CD16 microbeads (Miltenyi Biotec, Germany) and the $\mathrm{CD} 16^{+}$monocytes were magnetically separated on LS columns as mentioned previously (Section 2.3.2). The magnetically separated $\mathrm{CD} 16^{+}$monocytes were taken for cell culture, and the column effluent was collected and used for further isolation of $\mathrm{CD} 14^{+}$monocytes.

\subsubsection{Positive isolation of $\mathrm{CD} 14^{+}$monocytes}

The collected effluent with remaining $\mathrm{CD}_{1} 4^{+}$monocytes and lymphocytes was magnetically labelled with $\mathrm{CD} 14$ microbeads and the $\mathrm{CD} 14^{+}$monocytes were magnetically separated on LS columns as mentioned above (Section 2.3.2). The column effluent consisted of depleted lymphocytes, and was discarded. The isolated $\mathrm{CD} 14^{+}$monocytes were taken for cell culture.

\subsection{Monocyte culture and IFN- $\gamma$ priming}

The isolated monocytes were resuspended in complete $\mathrm{T}$ cell media (CTCM) and cultured in duplicate or triplicate in U-bottomed 96-well plates (BD Biosciences, USA) at a concentration of $1 \times 10^{5}$ cells per well for flow cytometry experiments, and at a concentration 
of $1 \times 10^{6}$ cells per well for qPCR work. The cells were incubated at $37^{\circ} \mathrm{C}$ in $5 \% \mathrm{CO}_{2}$ for 16 hours in the presence of $20 \mathrm{U} / \mathrm{ml} \mathrm{IFN}-\gamma$ (IFN- $\gamma$ priming).

\subsection{LPS stimulation}

The primed cells were stimulated with lipopolysaccharide from E.Coli (Sigma, USA) at a final concentration of $200 \mathrm{ng} / \mathrm{ml}$. The cells were then incubated at $37^{\circ} \mathrm{C}$ in $5 \% \mathrm{CO}_{2}$ for 24 hours. After the incubation, $100 \mu \mathrm{l}$ of supernatant was harvested and stored at $-20^{\circ} \mathrm{C}$ for the subsequent measurement of cytokine levels. The cells were then used for flow cytometry or qPCR.

\subsection{GA treatment}

For in vitro GA treatment, primed cells were treated with glatiramer acetate (Copaxone, Sanofi Aventis) at a final concentration of $100 \mu \mathrm{g} / \mathrm{ml}$ per $1 \times 10^{5}$ cells, in the presence or absence of LPS. The cells were then incubated at $37^{\circ} \mathrm{C}$ in $5 \% \mathrm{CO}_{2}$ for 24 hours. After the incubation, $100 \mu \mathrm{l}$ of supernatant was removed and stored at $-20^{\circ} \mathrm{C}$, for cytokine measurement at a later date. The cells were taken for flow cytometry or qPCR.

During in vivo GA treatment, MS patients received daily $20 \mathrm{mg}$ glatiramer acetate injections (Copaxone; Sanofi Aventis, Australia) for a minimum of six months prior to the blood collection.

\subsection{IC treatment in vitro}

Immune complexes (IC) were prepared as following. Human red blood cells (RBC) from blood group A (NZ Blood, Auckland, New Zealand) were mixed with intravenous immunoglobulin G (IVIG; NZ Blood, NZ) and incubated at room temperature for 30 minutes with constant rotation. The primed cells were treated with IC, consisting of a 1:100 dilution of IVIG and 10 RBC per monocyte, in the presence or absence of LPS. The cells were then incubated at $37^{\circ} \mathrm{C}$ in $5 \% \mathrm{CO}_{2}$ for 24 hours. After the incubation, $100 \mu 1$ of supernatant was

removed for the detection of cytokines, and stored at $-20^{\circ} \mathrm{C}$. The cells were then taken for flow cytometry or qPCR. 


\subsection{Flow cytometry}

\subsubsection{Staining and data acquisition}

Flow cytometry antibodies (Appendix 2) were pre-titrated in order to optimise the resolution between the positive and negative cell populations, and minimise background fluorescence. Cells were washed in FACS buffer, then stained with the optimised primary antibodies diluted in FACS buffer, in a total volume of 50 $\mu$. The samples were incubated for 15 minutes at room temperature, washed with FACS buffer, centrifuged at $700 \mathrm{x} \mathrm{g}$ for 5 minutes, and resuspended in FACS buffer. When biotinylated primary antibodies were used, cells were incubated for a further 15 minutes with streptavidin-conjugated fluorophores and then washed with FACS buffer. The stained cells were resuspended in FACS buffer for subsequent acquisition on a FACS Canto II flow cytometer using Diva software (BD Biosciences, USA). Data was analysed using FlowJo 5 software (Tree Star, Ashland, OR, USA).

\subsubsection{Gating strategies}

Live, singlet cells were gated using forward scatter properties (FSC-H and FSC-A). $\mathrm{CD} 45^{+}$leukocytes were gated from the total live cells. The monocytes were then selected from the $\mathrm{CD}_{4} 5^{+}$cells and were assessed to check the purity of monocytes. Monocytes were also either assessed for activation marker expression, or further divided into $\mathrm{CD} 14^{++} \mathrm{CD} 16^{-}$ and $\mathrm{CD} 14^{+} \mathrm{CD} 16^{+}$monocytes in order to evaluate marker expressions on the co-cultured $\mathrm{CD} 14^{+}$and $\mathrm{CD} 16^{+}$subsets (Appendices 8-11).

\subsection{Cytokine measurement}

\subsubsection{Extracellular cytokine detection by enzyme linked immunosorbent assay}

An enzyme linked immunosorbent assay (ELISA) was used to measure extracellular cytokine secretion according to the manufacturer's protocol. All of the primary and secondary antibodies were pre-titrated to find the optimal concentration for detection of cytokines. 96 well ELISA plates (BD Biosciences, USA) were coated with $50 \mu$ of primary, 
or capture, antibody in sodium phosphate buffer overnight at $4{ }^{\circ} \mathrm{C}$ or for 2 hours at room temperature (Appendix 3). The plates were then washed in wash buffer (PBS containing $0.05 \%$ Tween 20 (Sigma, USA)) and blocked with 100 $\mu$ l of 5\% FCS in PBS for 2 hours at room temperature. After washing, $50 \mu \mathrm{l}$ of standards or samples (culture supernatant) were added to the plate and incubated overnight at $4{ }^{\circ} \mathrm{C}$, or for 2 hours at room temperature. Following this incubation, the plate was washed and $50 \mu \mathrm{l}$ of biotinylated secondary, or detection, antibody was added and incubated for 1 hour at room temperature. After washing, $50 \mu \mathrm{l}$ of streptavidin conjugated horseradish peroxidase was added and incubated for 1 hour at room temperature. The plates were then washed and $100 \mu$ of tetramethyl benzidine mixture was added. After sufficient color development, the reaction was stopped by the addition of $100 \mu \mathrm{l}$ of stop solution (0.18 M sulphuric acid). Absorbance values were measured at $450 \mathrm{nM}$ using a VersaMax plate reader (Molecular Devices, Sunnyvale, CA, USA). A standard curve was constructed from the absorbance values of the standards and then used to calculate the sample cytokine concentrations. The detection limit for individual cytokines ranged from $15-100 \mathrm{pg} / \mathrm{ml}$, as indicated on the manufacturer's datasheet.

\subsubsection{Extracellular cytokine detection by cytometric bead array}

A human inflammatory cytokines cytometric bead array (CBA) kit (BD Biosciences) was used to detect extracellular cytokine secretion. The cytokines were quantified using a human inflammatory cytokine cytometric bead array (CBA) kit (BD Biosciences, San Jose, CA, USA). The CBA kit consists of six bead populations with distinct fluorescence intensities that can be resolved in the red FL-3 channel. Each population is coated with capture antibodies specific for IL-1b, IL-6, IL-8, IL-10, IL-12p70 or TNF-a. After these beads are incubated with test samples or standards, phycoerythrin (PE)-conjugated cytokinespecific detection antibodies are added to enable the cytokine-bound beads to be distinguished from unbound beads. The PE fluorescence intensity of the resulting sandwich complexes can then be detected in the yellow FL-2 channel during flow cytometry and compared to a range of standards to ascertain the cytokine concentrations. $25 \mu 1$ of undiluted sample and duplicate standards ranging from $20 \mathrm{pg} / \mathrm{ml}$ to $5000 \mathrm{pg} / \mathrm{ml}$ were incubated with 25 $\mu l$ of the mixed beads for 1.5 hours. At the end of the incubation, the samples were washed twice with wash buffer and all but $50 \mu \mathrm{l}$ of supernatant was aspirated. $25 \mu \mathrm{l}$ of the PEconjugated anti-cytokine antibodies were then added to each sample. After a 1.5 hour incubation, the samples were washed twice, resuspended in $200 \mu 1$ wash buffer. The samples 
were acquired on a FACS Canto II flow cytometer and the data analyzed using FCAPArray software (BD Biosciences, USA). The detection limit for the individual cytokines ranged from 2-20 pg/ml, as indicated on the manufacturer's datasheet.

\subsubsection{Intracellular cytokine detection by flow cytometry}

Intracellular staining of cultured monocytes was performed in order to detect intracellular cytokine production. The cells were incubated with LPS, GA or IC in the presence of monensin (BD Biosciences, USA) at a final concentration of $0.67 \mu \mathrm{l} / \mathrm{ml} / 10^{6}$ cells for 22 hours at $37^{\circ} \mathrm{C}$. The cells were then harvested, washed with FACS buffer and centrifuged at $400 \mathrm{x} \mathrm{g}$ for 5 minutes. Extracellular staining of the cells was performed by incubating with fluorescent anti-CD antibodies (Biolegend, San Diego, CA, USA) at room temperature in the dark for 15 minutes (Appendix 4). The cells were fixed in $100 \mu 1$ of 4\% paraformaldehyde at room temperature in the dark for 20 minutes. The cells were then permeabilized using $200 \mu \mathrm{l}$ of $0.1 \%$ saponin buffer and incubated at room temperature in the dark for 15 minutes. Intracellular staining was performed using fluorescent anti-cytokine antibodies (Biolegend, USA) diluted in saponin buffer and incubated with the cells at room temperature in the dark for 30 minutes. Finally, the cells were acquired on a FACS Canto II flow cytometer (BD Biosciences, USA) and the data was analyzed using FlowJo 5 software (Tree Star, USA).

\subsection{MTT}

A colorimetric MTT assay was performed to assess the cytotoxicity of the treatments. The treated cells were incubated with $20 \mu \mathrm{l}$ of 3-(4,5-dimethylthiazol-2-yl)-2.5diphenyrtetrazolium bromide in dPBS (MTT solution; Sigma, USA) at $37^{\circ} \mathrm{C}$ in $5 \% \mathrm{CO}_{2}$ for 2 hours. Then, $100 \mu \mathrm{l}$ of MTT solubiliser was added and the cells were incubated for a further 16 hours. The OD expression of MTT was measured by absorbance at $570 \mathrm{~nm}$ on a Versamax plate reader (Molecular Devices, USA). A colour change of the MTT solution, added to the stimulated cells, from yellow to purple, represents good metabolic activity of monocytes with no cytotoxicity upon stimulation with LPS, shown by high OD fluorescence. However, if the stimulants are cytotoxic, the metabolic activity of cells is decreased and little or no colour change is observed[182]. 


\subsection{Validation of data by $q P C R$}

\subsection{1 cDNA synthesis}

cDNA was synthesized using SuperScript ${ }^{\mathrm{TM}}$ III First-Strand Synthesis Supermix kit (Invitrogen, Carlsbad, CA, USA) accordancing the manufacturer's protocol. Briefly, the DNase-treated RNA $(1 \mu \mathrm{g})$ was mixed with $1 \mu \mathrm{L}$ primer $\left.(50 \mu \mathrm{M} \text { oligo(dT) })_{20}\right), 1 \mu \mathrm{L}$ annealing buffer, and nuclease-free water (to make a total volume of $8 \mu \mathrm{L}$ ), and incubated at $65^{\circ} \mathrm{C}$ for 5 minutes. After cooling the reaction tube on ice for at least 1 minute, the samples were mixed with $2 \mathrm{X}$ first-strand reaction mix $(10 \mu \mathrm{L})$ and SuperScript ${ }^{\mathrm{TM}}$ III/RNaseOUT enzyme mix (2 $\mu \mathrm{L}$ ) and incubated at $50^{\circ} \mathrm{C}$ for 50 minutes. The reaction was terminated by heating the sample to $85^{\circ} \mathrm{C}$ for 5 minutes. The cDNA was stored at $-80^{\circ} \mathrm{C}$ until further use.

\subsubsection{Quantitative real-time PCR}

Expression levels of the mRNA for cell surface markers, cytokines, and house-keeping genes were determined using a quantitative PCR method. Common house-keeping genes, such as cyclophilin and $\beta$-actin, were used. All primers were manufactured by Integrated DNA Technologies (Coralville, IA, USA) (Appendix 5). To quantify the expression levels of each of the genes, singleplex reaction mixes were prepared containing a single set of primers at validated concentrations $(200 \mathrm{nM})$ and the reagents supplied in the ' $\mathrm{SYBR}^{\circledR}$ GreenER ${ }^{\mathrm{TM}} \mathrm{qPCR}$ SuperMix Universal Kit' (Invitrogen, USA), according to the manufacturer's protocol. Samples were prepared in duplicate by adding the cDNA sample (200 ng) to the prepared reaction mix (total volume of $52 \mu \mathrm{L}$ ), and then transferring two $25 \mu \mathrm{L}$ aliquots, each containing $100 \mathrm{ng}$ of cDNA, into $0.2 \mathrm{~mL}$ optical PCR tubes. The amplification reaction was run on an iCycler real-time PCR detection system (Bio-Rad, Hercules, CA, USA) under the following conditions: 1 cycle of $50^{\circ} \mathrm{C}$ for 2 minutes, 1 cycle of $95^{\circ} \mathrm{C}$ for 2 minutes, 40 cycles of $95^{\circ} \mathrm{C}$ for 15 seconds, and $60^{\circ} \mathrm{C}$ for 30 seconds. Controls included samples that underwent reverse transcription-PCR with the exclusion of SuperIII/RNaseouT enzyme mix in order to check the effectiveness of the DNase treatment and reaction mixtures that did not contain a template. The PCR amplification efficiency for each set of primers was evaluated using a standard curve created with a serially diluted cDNA sample. The mRNA expression was analysed as $2^{-\Delta \Delta C q}$ as described[183]. 


\subsection{Hiearchial cluster analysis}

The hierarchial cluster analysis (HCA) was done by using HCE3,5 and MatLabR2012a softwares (Math Works Inc, USA). An average linkage method and row and column clustering directions were used. In order to measure similarities and distances, the Pearson correlation coefficient was selected, whereas the mosaic mapping method was chosen for display of the data. The statistical analysis between the clustered groups was performed by using MANOVA (multivariate analysis of variance) with Wilks' Lambda post hoc test.

\subsection{Statistical analysis and graphs}

Statistical power analysis was performed in order to define a minimum number of volunteers to be involved in this study. The power analysis considered a variability between the subjects and a variability that may rise due to conducting experiments in different days, and the minimum number of subjects to recruit was defined as 20 in each group. Statistical analyses of the experimental results and graphs were performed using GraphPad Prism 5 (GraphPad Software Inc., La Jolla, CA, USA) and Microsoft Excel 2010 softwares (Microsoft Corporation, Redmond, WA, USA). The descriptive statistics were created in order to make histograms and assume the normal distribution of the data. Student's t-test was used to determine statistical significance when comparing two treatment groups. One-way ANOVA with Dunnett's post-hoc test was used when comparing three or more groups. 
Chapter 3.

Characterization of ex vivo monocytes from MS patients 


\subsection{INTRODUCTION}

Monocytes, together with $\mathrm{T}$ cells, promote inflammation in MS by activating other immune cells and directly damaging neurons[1, 76]. The circulating blood monocytes of MS patients have inflammatory characteristics and are similar to classically activated monocytes. These characteristics include the upregulated expression of activation markers such as CD40, CD86, HLA-DR[53] and inflammatory cytokines[184]. The upregulation of these activation markers and cytokines leads to the stimulation and sustained activation of inflammatory Th1 and Th17 cells, which are well known drivers of the inflammation in MS[43, 63, 185]. Monocytes have two main subsets with distinct phenotypes[41], namely CD14 ${ }^{+}$and $\mathrm{CD}_{16}{ }^{+}$ monocytes; however, the role of these subsets in the $\mathrm{T}$ cell dysregulation (i.e. sustained activation of Th1 and Th17 cells) that occurs during MS, is unclear.

The $\mathrm{CD} 6^{+}$monocytes are increased in number during MS, whereas the number of $\mathrm{CD} 14^{+}$monocytes is decreased[97]. While it is clear that the $\mathrm{CD}_{1} 4^{+}$and $\mathrm{CD} 16^{+}$monocytes have unique phenotypes and differ in their function during inflammation[79, 80, 82-84], the role of $\mathrm{CD}_{1} 4^{+}$and $\mathrm{CD}^{+} 6^{+}$monocytes in MS has not yet been described. This study characterizes the phenotype of inflammatory monocytes and subsets during MS.

\subsection{AIMS}

This chapter aims to characterize the phenotype of monocytes, including CD14 $4^{+}$and $\mathrm{CD}_{1} 6^{+}$monocyte subsets, in a New Zealand population of MS patients and healthy subjects. 


\subsection{RESULTS}

\subsubsection{Optimization of monocyte isolation}

\subsubsection{Isolation of total monocytes}

Two different methods of magnetic separation were used to isolate monocytes from peripheral blood mononuclear cells (PBMCs): 1) purification with Dynabeads ${ }^{\circledR}$, and 2) purification with Microbeads ${ }^{\circledR}$. In both methods, magnetic beads specific to human CD14 antigen were used. Although monocyte separation using CD14 Dynabeads resulted in a highly pure monocyte population, subsequent experiments using flow cytometry showed that cell surface markers were detectable only at minimal levels and with a high variability (Figure 3.1). Dynabeads are relatively large in size $(4.5 \mu \mathrm{M})$ and are easily visible under a light microscope. Mechanical blockage of monocyte receptors by firmly attached Dynabeads can thus impair the subsequent binding of fluorescent antibodies. Attached Dynabeads were visible under the light microscope throughout all of the monocyte culture and stimulation steps (data not shown). In addition, when the expression of markers on Dynabead-isolated monocytes was compared to those of monocytes before Dynabead isolation (i.e. within PBMC cultures), the expression was minimal and highly variable in the Dynabead-isolated monocytes (Figure 3.1). In addition, classical activation of monocytes with IFN- $\gamma$ priming and LPS stimulation did not induce expression of CD40 in the Dynabead-isolated monocytes (Figure 3.2). Therefore, magnetic isolation with Dynabeads was not considered an appropriate method for the isolation of monocytes for these studies. 
a.
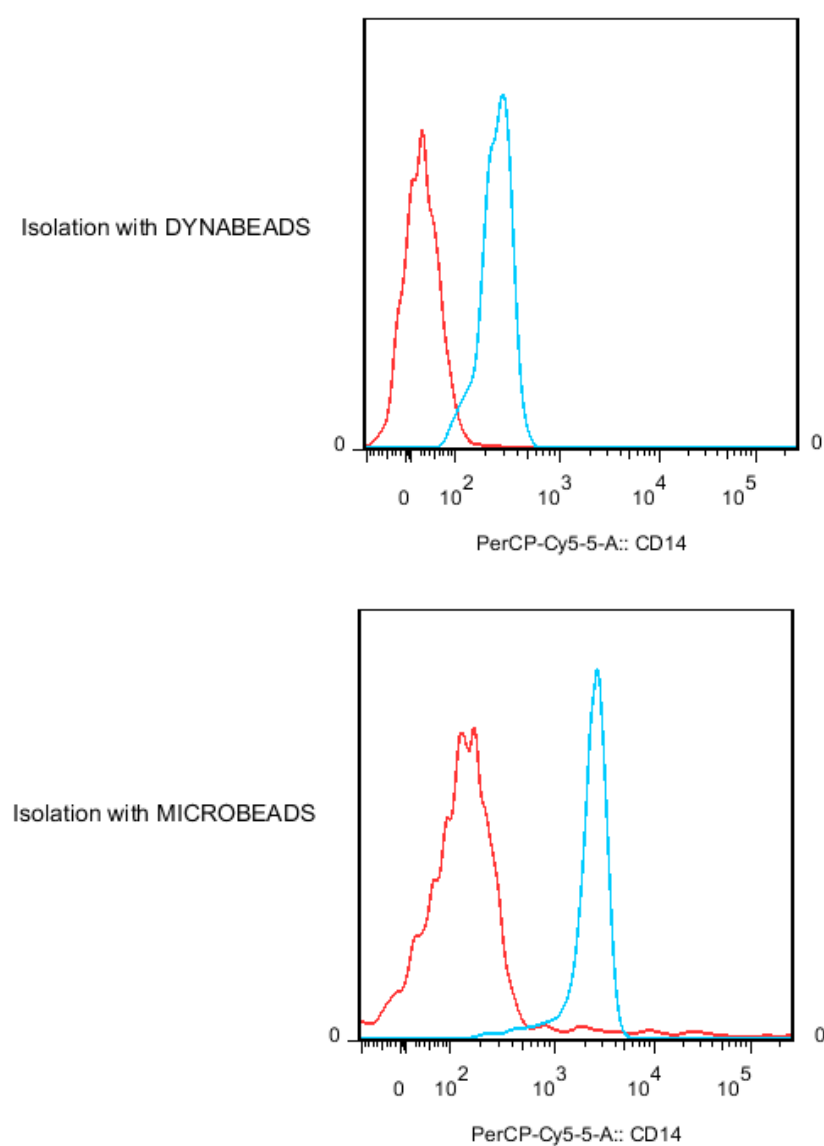

b.
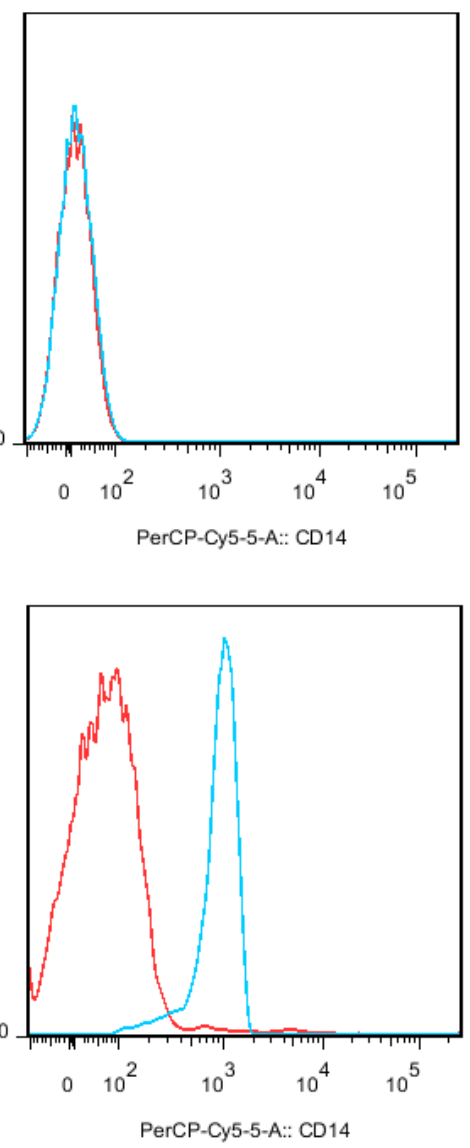

Figure 3.1. Microbead-isolated monocytes express CD14.

Total monocytes were isolated from PBMC using CD14 Dynabeads or CD14 Microbeads. Isolated monocytes were stained with PerCP-Cy5,5-conjugated anti-CD14 antibody (blue) or isotype control (red). The expression of CD14 was assessed by flow cytometry. Data was analyzed on FlowJo software and the monocytes were gated on $\mathrm{CD}_{4} 5^{+}$live singlet cells. (a) CD14 expression on monocytes before isolation, i.e. in PBMC. (b) CD14 expression on monocytes after positive isolation with Dynabeads or Microbeads. 
a.

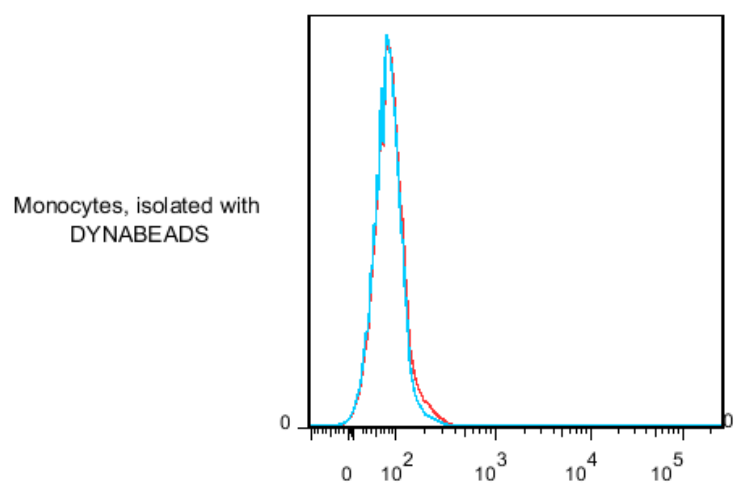

PE-A:: CD40

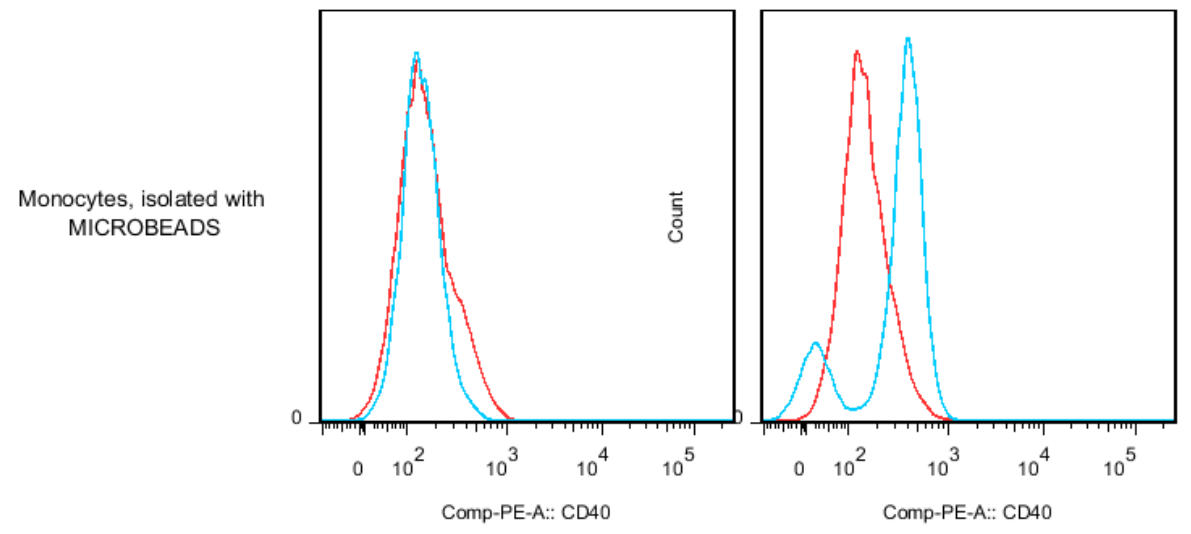

Figure 3.2. Microbead-isolated monocytes express CD40 upon LPS stimulation.

Total monocytes were isolated from PBMC using CD14 Dynabeads or CD14 Microbeads. Isolated monocytes were primed with IFN- $\gamma(20 \mathrm{U} / \mathrm{ml})$ overnight and cultured in medium with or without LPS (200 ng/ml) for 24 hours. The monocytes were harvested and stained with PE-conjugated anti-CD40 antibody (blue) or isotype control (red), and the expression of CD40 assessed by flow cytometry. Data was analyzed on FlowJo software and the monocytes were gated on $\mathrm{CD} 5^{+}$live singlet cells. (a) CD40 expression on monocytes treated with medium only. (b) CD40 expression on monocytes stimulated with LPS. 
In contrast to Dynabeads, magnetic Microbeads are bio-degradable, have a size of $50 \mathrm{~nm}$ (which is 90-fold smaller than Dynabeads) and were not visible under light microscope within 24 hours of culture. Microbead-isolated monocytes had a similar expression of markers as monocytes within PBMCs (i.e. before magnetic separation) and gave a highly pure yield of isolated monocytes (Figure 3.3), indicating that Microbeads do not interfere with the binding of fluorescent antibodies. In addition, Microbead-isolated monocytes did not show any unspecific activation, as no cytokine production was observed when Microbeadisolated monocytes were cultured in media without additional stimulants (Chapter 4.3.2). Thus, the positive selection of monocytes using magnetic Microbeads, was chosen as the best method for monocyte isolation.

a.

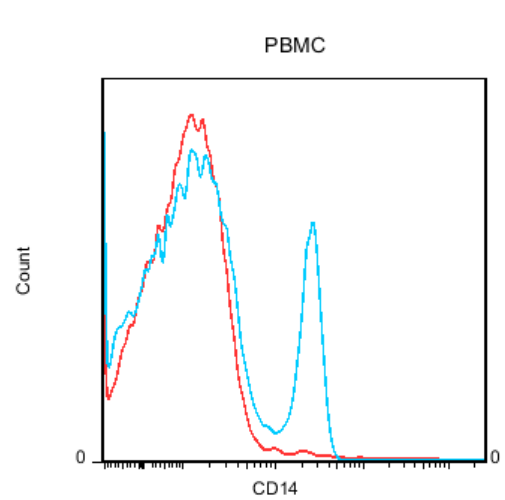

b.

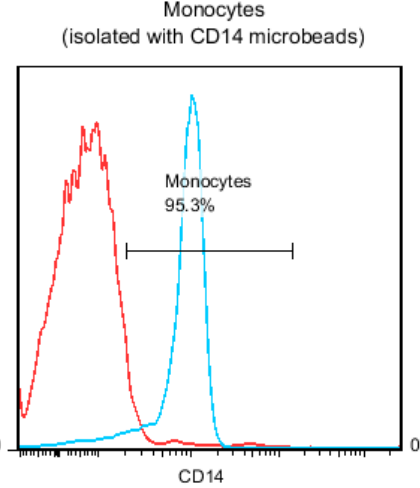

c.

Monocyte-depleted PBMC

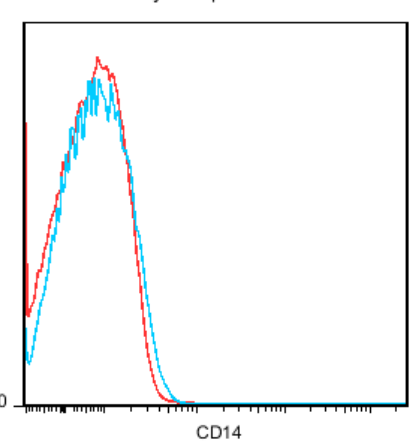

Figure 3.3. More than $95 \%$ of Microbead-isolated cells express CD14.

Total monocytes were isolated from PBMC using CD14 Microbeads. Isolated monocytes were stained with PerCP-Cy5,5-conjugated anti-CD14 antibody (blue) or isotype control (red). The expression of CD14 was assessed by flow cytometry. Data was analyzed on FlowJo software and the monocytes were gated on CD $45^{+}$ live singlet cells. (a) CD14 expression on monocytes before isolation, i.e. in PBMC. (b) CD14 expression on monocytes after positive isolation with Microbeads. (c) CD14 expression on monocyte-depleted PBMC, i.e. lymphocytes. 


\subsubsection{Isolation of monocyte subsets}

In this study, to isolate monocyte subsets, PBMC were depleted of all non-monocyte cells that express CD16, namely neutrophils and NK cells. CD15 and CD56 microbeads were used in order to deplete these populations as they express CD15 (neutrophils)[186] and CD56 antigens (NK cells)[187] on their surfaces. $\mathrm{CD}^{+} 6^{+}$monocytes were then positively isolated using CD16 Microbeads. The $\mathrm{CD}^{+} 4^{+} \mathrm{CD} 16^{-}$population $\left(\mathrm{CD} 14^{+}\right.$monocytes) was then positively isolated from the sample using CD14 Microbeads. The remaining cells were primarily $\mathrm{CD}^{+}$lymphocytes (Figure 3.4).

a.

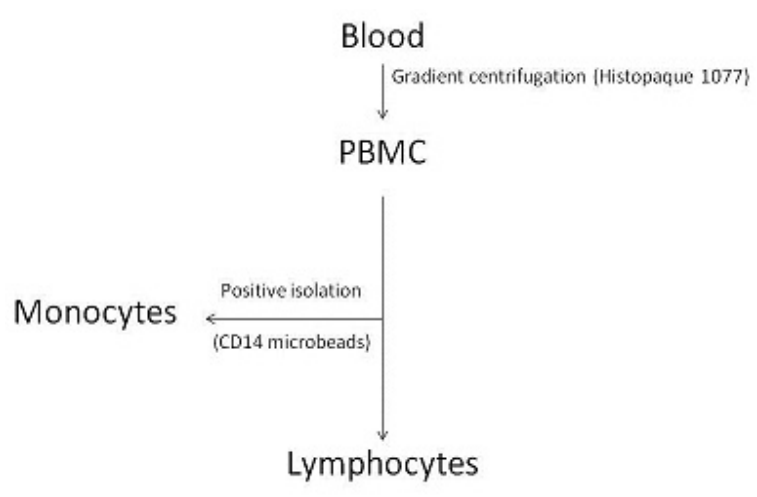

b.

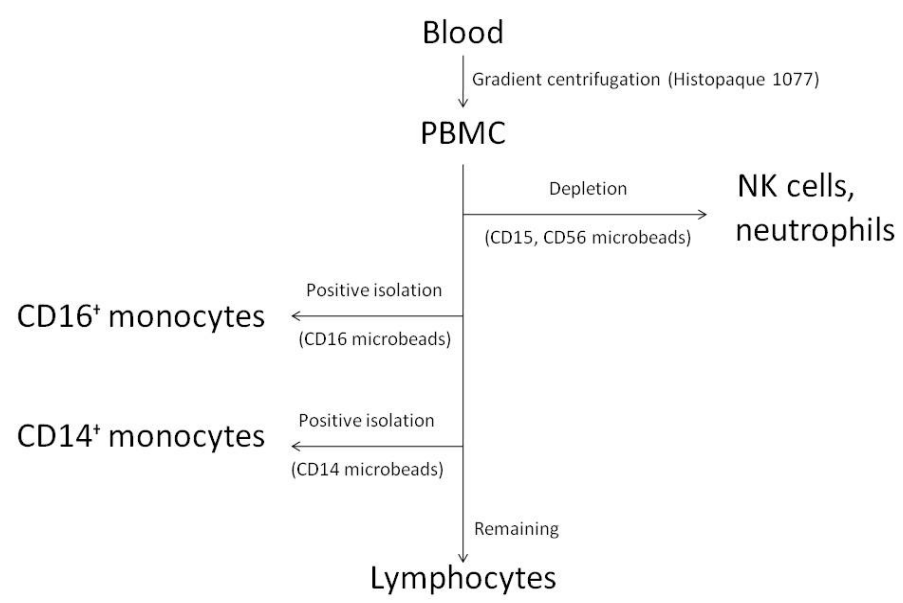

Figure 3.4. Work flow for monocyte and monocyte subset isolation.

PBMC were isolated from human peripheral blood by gradient centrifugation with Histopaque 1077. Monocytes were isolated from PBMC using magnetic Microbeads. (a) Total monocytes were positively isolated from PBMC using CD14 Microbeads. The remaining cells mainly consisted of lymphocytes. (b) Monocyte subsets were positively isolated from PBMC using CD16 and CD14 Microbeads after depletion of CD16 ${ }^{+} \mathrm{NK}$ cells and granulocytes by using CD15 and CD56 Microbeads. The remaining cells mainly consisted of lymphocytes. 
The purity of monocytes was assessed by flow cytometry, based on the expressions of CD14, CD16 and CD11b markers. Using the methods described above, we achieved greater than $95 \%$ purity of total monocytes (Figure 3.3) and in both $\mathrm{CD}_{1} 4^{+}$and $\mathrm{CD}_{16}{ }^{+}$subsets (Figure 3.5).

a.

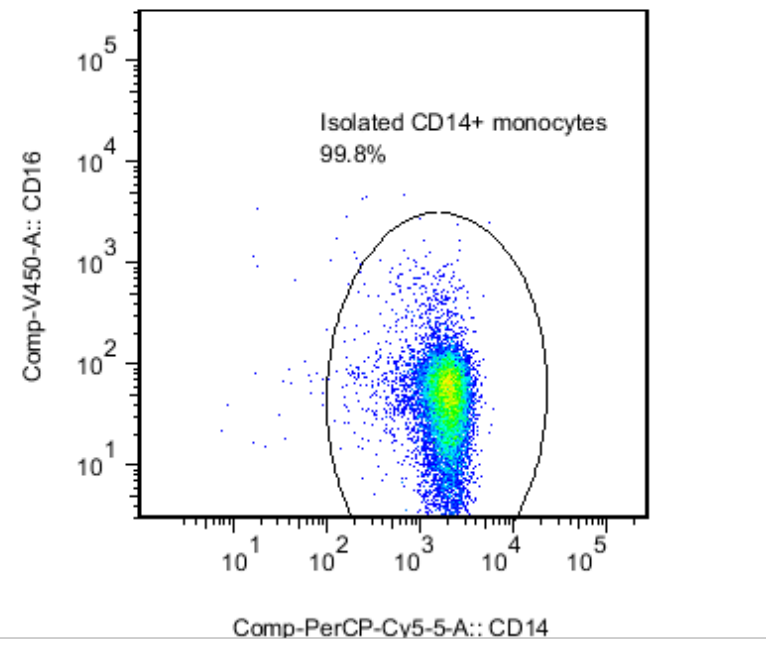

b.

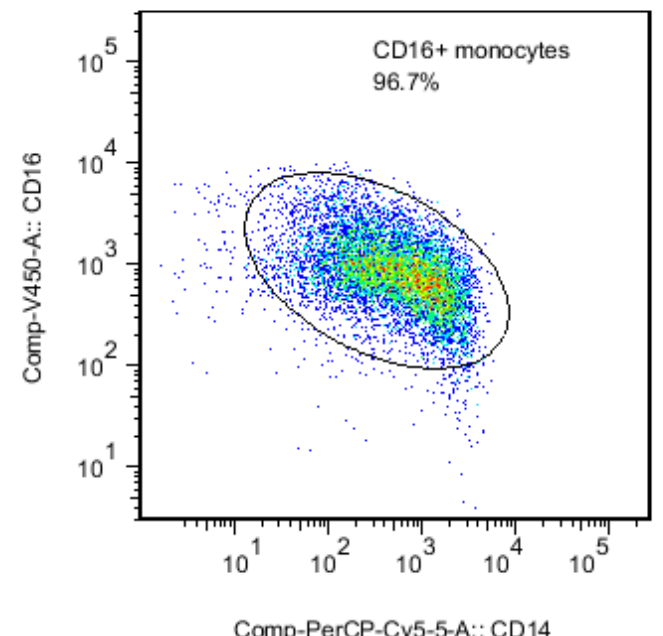

Figure 3.5. Isolated monocyte subsets had above $95 \%$ purity.

Monocytes were isolated from PBMC using CD14 and CD16 Microbeads. Isolated monocytes were stained with PerCP-Cy5,5-conjugated anti-CD14 and V450-conjugated anti-CD16 antibodies. The purity of monocyte subsets was assessed based on the expressions of CD14 and CD16 by flow cytometry. (a) CD14 and CD16 marker expression on isolated $\mathrm{CD} 14^{+}$monocyte subset. (b) CD14 and CD16 marker expression on isolated CD16 ${ }^{+}$monocyte subset. 
The monocyte subset identity was verified using expression of CD11b, CD14 and CD16 cell surface markers. While the $\mathrm{CD} 14^{+}$subset of monocytes have a high expression of CD11b and CD14 (the hallmarks of monocytes), the $\mathrm{CD}^{+} 6^{+}$subsets have an intermediate level of expression of CD16 (FcyRIII) and CD14. The isolated $\mathrm{CD}_{14}^{+}$monocytes had high expression levels of both CD11b and CD14, and undetectable levels of CD16 expression in both healthy and MS groups. Therefore, the purified $\mathrm{CD}_{1}{ }^{+}$monocytes were $\mathrm{CD} 11 \mathrm{~b}^{++} \mathrm{CD} 14^{++} \mathrm{CD} 16^{-}$monocytes (Figure 3.6). In contrast, the isolated CD16 ${ }^{+}$monocytes had low expression levels of CD11b and CD14, and medium expression levels of CD16 in both groups (Figure 3.7). Therefore, $\mathrm{CD}^{+} 6^{+}$monocytes were $\mathrm{CD} 11 \mathrm{~b}^{+} \mathrm{CD} 14^{+} \mathrm{CD} 16^{+}$ monocytes (Figure 3.8).

a.

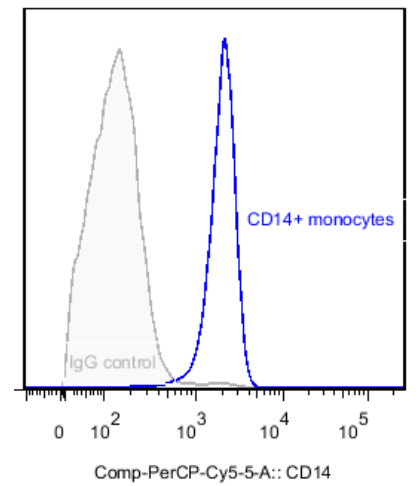

b.

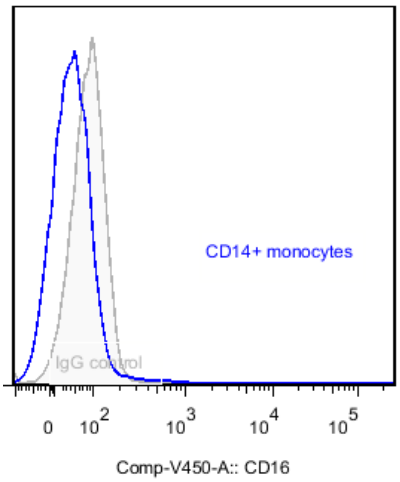

c.

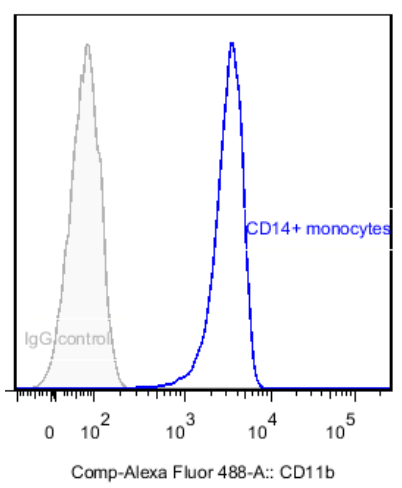

Figure 3.6. $\mathrm{CD}^{+}{ }^{+}$monocytes express high levels of CD11b and CD14 and no CD16.

$\mathrm{CD} 14^{+}$monocytes were isolated from PBMC depleted of NK cells, granulocytes and CD16 $6^{+}$monocytes by using CD14 Microbeads. The isolated CD14 $4^{+}$monocytes were stained with PerCP-Cy5,5-conjugated anti-CD14, AF488-conjugated CD11b and V450-conjugated anti-CD16 antibodies (blue) or isotype control (grey). The expression of CD14 (a), CD16 (b), and CD11b (c) on the CD14 ${ }^{+}$monocyte subset. Data were collected on flow cytometry using the gating strategy in appendix 10 . 
a.

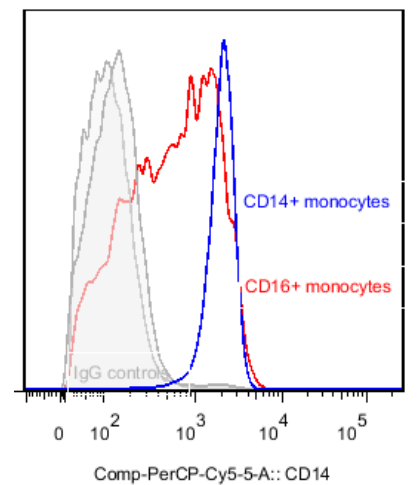

b.

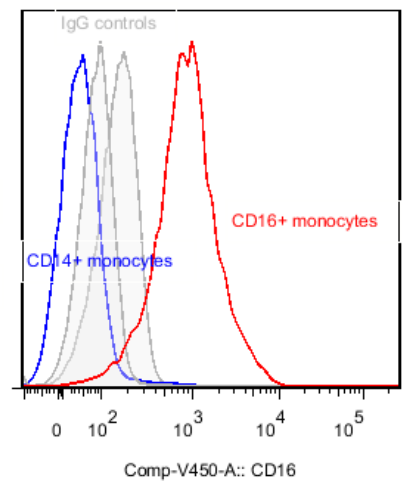

c.

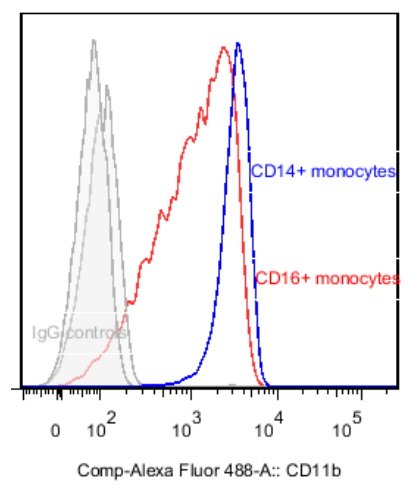

Figures 3.7. Expression of CD11b, CD14 and CD16 on monocyte subsets.

$\mathrm{CD} 16^{+}$monocytes were isolated from PBMC depleted from NK cells and granulocytes using CD16 Microbeads. The $\mathrm{CD} 14^{+}$monocytes were then isolated from the remaining PBMC by using CD14 Microbeads. The isolated $\mathrm{CD}_{14}{ }^{+}$and $\mathrm{CD}_{16}{ }^{+}$monocytes were stained with PerCP-Cy5,5-conjugated anti-CD14, AF488-conjugated $\mathrm{CD} 11 \mathrm{~b}$ and V450-conjugated anti-CD16 antibodies (blue or red) or isotype control (grey). The expression of CD14 (a), CD16 (b), and CD11b (c) CD14 (blue) and CD16 (red) monocyte subsets. The histograms represent the same data for the $\mathrm{CD} 14^{+}$monocytes as in Figure 3.6. Data were collected on flow cytometry using the gating strategies in appendices 10,11 .

a.

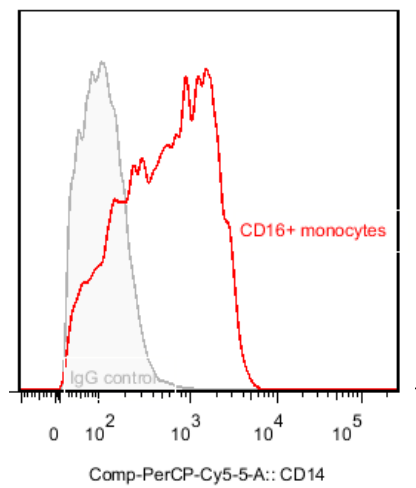

b.

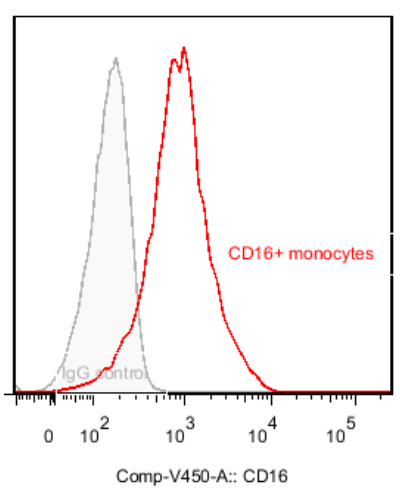

c.

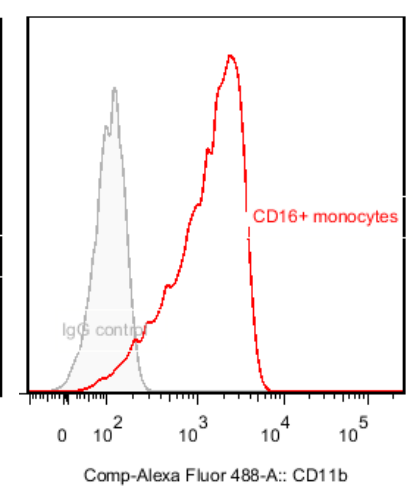

Figure 3.8. $\mathrm{CD}^{+}{ }^{+}$monocytes express medium levels of CD11b and CD14 and CD16.

$\mathrm{CD} 16^{+}$monocytes were isolated from PBMC depleted from NK cells and granulocytes using CD16 Microbeads. The isolated $\mathrm{CD}_{16}{ }^{+}$monocytes were stained with PerCP-Cy5,5-conjugated anti-CD14, AF488-conjugated $\mathrm{CD} 11 \mathrm{~b}$ and V450-conjugated anti-CD16 antibodies (red) or isotype control (grey). The expression of CD14 (a), $\mathrm{CD} 16$ (b), and CD11b (c) on CD16 $6^{+}$monocyte subset. The histograms represent the same data for the CD16 ${ }^{+}$ monocytes as in Figure 3.7. Data were collected on flow cytometry using the gating strategy in appendix 11. 


\subsubsection{Proportion of monocytes from healthy and MS groups}

In MS patients ( $\mathrm{n}=20)$, approximately $11-16 \%$ of the PBMCs were monocytes, based on the expression of $\mathrm{CD} 11 \mathrm{~b}$ and $\mathrm{CD} 14$, which is similar to the proportion of monocytes in healthy subjects $(n=26)(10-20 \%)$. However, the proportions of $\mathrm{CD}^{+} 4^{+}$and $\mathrm{CD} 16^{+}$monocyte subsets were altered in MS patients, such that the proportion of CD14 ${ }^{+}$monocytes was lower in MS patients $(73.6 \% \pm 17$ (SD)) compared to healthy subjects (92\% \pm 4 (SD)). Conversely, the proportion of $\mathrm{CD}_{1} 6^{+}$monocytes was significantly increased (five-fold) in MS patients $(26.2 \% \pm 17.3(\mathrm{SD}))$ compared to the healthy subjects $(5.1 \% \pm 2.7(\mathrm{SD}))$.

\subsubsection{Activation state of monocytes from healthy and MS groups}

In order to investigate the activation state of monocytes in MS patients in comparison to healthy subjects, the expression of activation markers on ex vivo isolated total monocytes was assessed by flow cytometry. In our study, monocytes from MS patients had significantly higher expression of the activation markers CD40, CD86, HLA-DR and CCR2, whereas the increase in CD64 was not significant (Figure 3.9). This finding indicates that monocytes in the MS group were more highly activated than those in the healthy group.

When the expression of markers on the isolated $\mathrm{CD}_{1} 4^{+}$subset was compared, the expression of CD86, HLA-DR and CD64 was upregulated on CD14 ${ }^{+}$monocytes from MS patients, while the expression of CD40 was downregulated (Figure 3.10). No difference was found in the expression of CCR2. In the $\mathrm{CD}_{1} 6^{+}$monocytes from MS patients, however, HLA-DR, CD64 and CCR2 were more highly expressed than in healthy group, whereas the expression of CD40 and CD86 was non-significantly higher in this subset (Figure 3.11). Together taken, both $\mathrm{CD} 14^{+}$and $\mathrm{CD}_{1} 6^{+}$monocytes from MS patients have characteristics of classically activated cells, as shown by the increased expression of all the activation markers assessed. 
a.

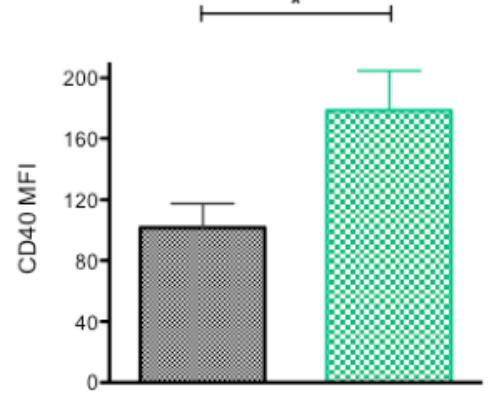

d.

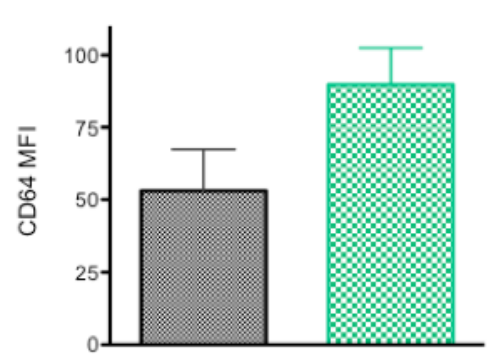

b.

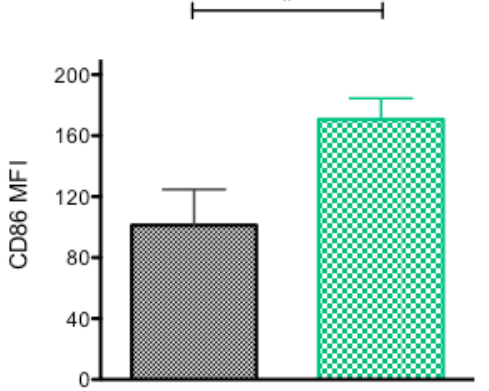

e.

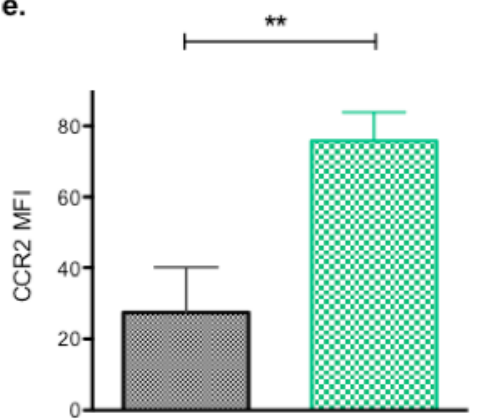

c.

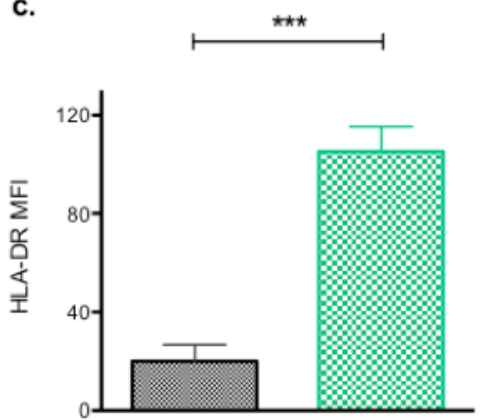

Healthy subjects

MS patients

Figure 3.9. Total monocytes from MS patients had high expression of activation markers.

Total $\mathrm{CD} 11 \mathrm{~b}^{+} \mathrm{CD} 14^{+}$monocytes were isolated from PBMC using CD14 Microbeads. Isolated monocytes were stained with fluorescent flow antibodies and the expression was assessed by flow cytometry. (a) The expression of CD40 on total monocytes. (b) The expression of CD86 on total monocytes. (c) The expression of HLA-DR on total monocytes. (d) The expression of CD64 on total monocytes. (e) The expression of CCR2 on total monocytes. Shown are the means and SD of 20 individuals in each of the subject groups. The statistical analysing was done by using Student's t-test to compare 2 groups. $* \mathrm{P}<0.01 ; * * \mathrm{P}<0.005 ; * * * \mathrm{P}<0.001$. Data were collected on flow cytometry using the gating strategy in appendix 8 . 
a.

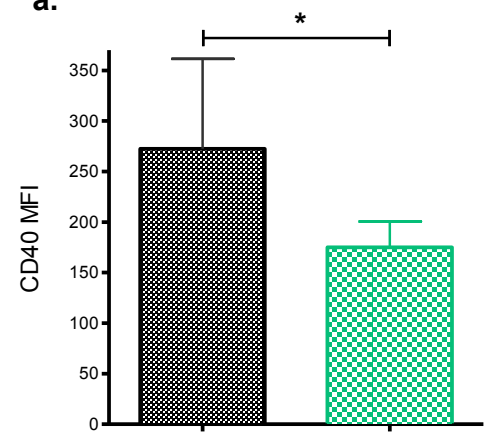

d.

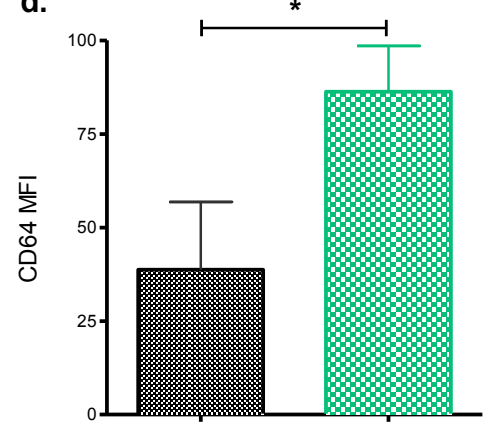

b.
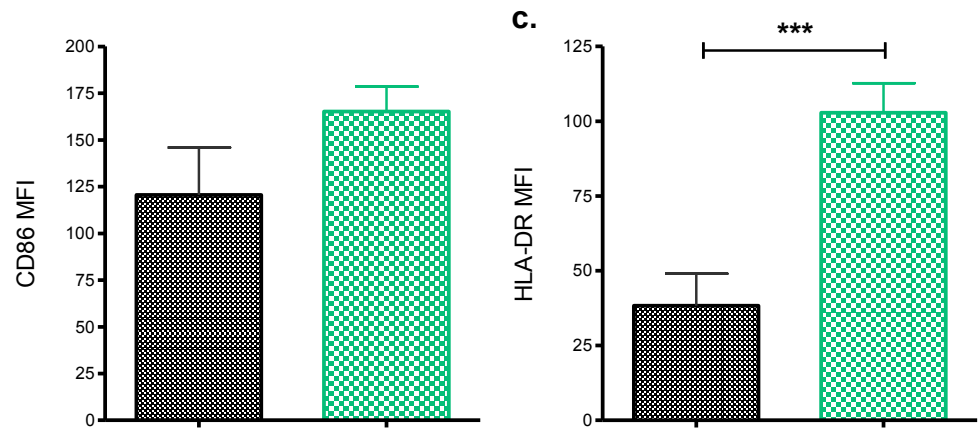

e.

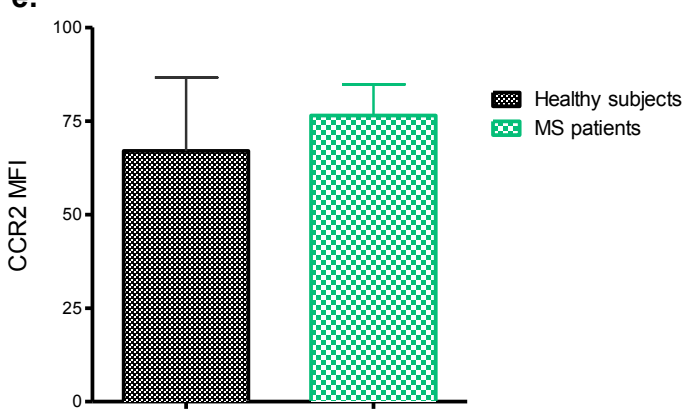

Figure 3.10. CD14 ${ }^{+}$monocytes from MS patients had high expression of CD86, HLA-DR and CD64.

Total monocytes were isolated from PBMC using CD14 Microbeads. Isolated monocytes were stained with fluorescent flow antibodies and the expression was assessed by flow cytometry. $\mathrm{CD} 14^{++} \mathrm{CD} 16^{-} \mathrm{CD} 11 \mathrm{~b}^{++}$ monocytes were gated out from the total monocyte populations on flow cytometry data, based on the expression of CD11b, CD14 and CD16. (a) The expression of CD40 on CD14 monocytes. (b) The expression of CD86 on CD14 $4^{+}$monocytes. (c) The expression of HLA-DR on CD14 $4^{+}$monocytes. (d) The expression of CD64 on CD14 monocytes. (e) The expression of CCR2 on CD14 ${ }^{+}$monocytes. Shown are the means and SD of 20 individuals in each of the subject groups. The statistical analysing was done by using Student's t-test to compare 2 groups. $* \mathrm{P}<0.01 ; * * * \mathrm{P}<0.001$. Data were collected on flow cytometry using the gating strategy in appendix 8 . 
a.

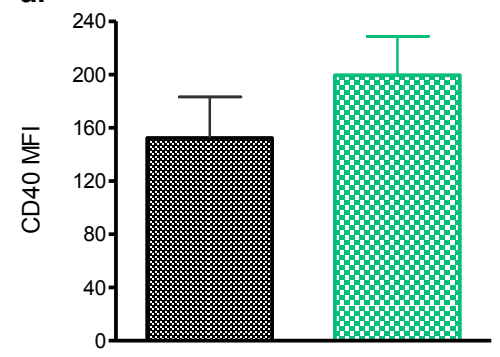

d.

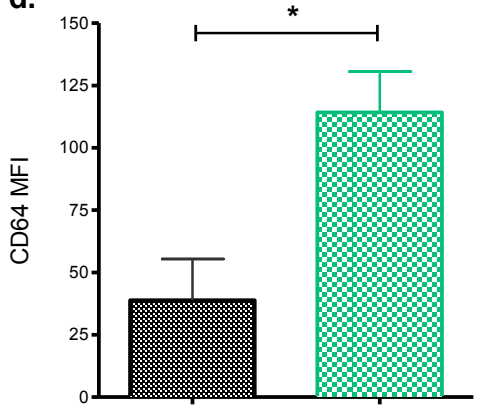

b.

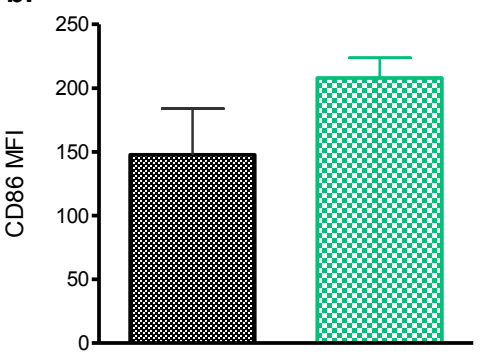

c.

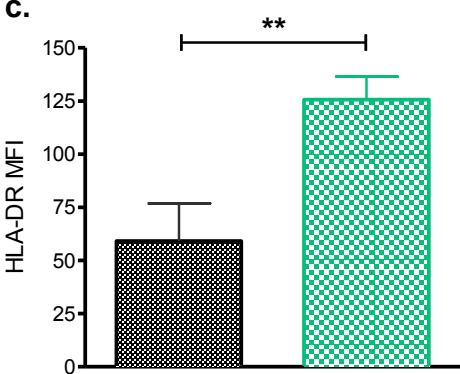

e.

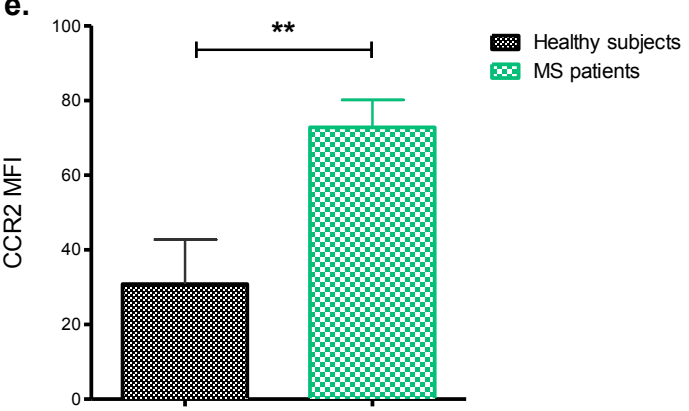

Figure 3.11. CD16 ${ }^{+}$monocytes from MS patients had high expression of activation markers.

Total monocytes were isolated from PBMC using CD14 Microbeads. Isolated monocytes were stained with fluorescent flow antibodies and the expression was assessed by flow cytometry. $\mathrm{CD} 14^{+} \mathrm{CD} 16^{+} \mathrm{CD} 11 \mathrm{~b}^{+}$ monocytes were gated out from the total monocyte populations on flow cytometry data, based on the expression of CD11b, CD14 and CD16. (a) The expression of CD40 on CD16 monocytes. (b) The expression of CD86 on CD $16^{+}$monocytes. (c) The expression of HLA-DR on CD16 monocytes. (d) The expression of CD64 on CD16 ${ }^{+}$ monocytes. (e) The expression of CCR2 on CD16 ${ }^{+}$monocytes. Shown are the means and SD of 20 individuals in each of the subject groups. The statistical analysing was done by using Student's t-test to compare 2 groups. $* \mathrm{P}<0.01 ; * * \mathrm{P}<0.005$. Data were collected on flow cytometry using the gating strategy in appendix 8 . 


\subsubsection{Comparison between $\mathrm{CD}^{+}{ }^{+}$and $\mathrm{CD}^{+}{ }^{+}$subset phenotypes}

In healthy subjects, CD14 $4^{+}$monocytes had higher expression of CD40 and CCR2 and lower expression of CD86 and HLA-DR, than the CD16 ${ }^{+}$monocytes, while having a similar expression of CD64 (Figure 3.12), when the expressions are compared between the CD14 ${ }^{+}$ and $\mathrm{CD} 16^{+}$monocytes. In MS patients, however, the expressions of activation markers were significantly altered, and the $\mathrm{CD}_{1} 6^{+}$monocytes had higher expression of all the activation markers, assessed in this study (Figure 3.13.a-d), except for CCR2 (Figure 3.13.e). The expression of CCR2 in the $\mathrm{CD} 16^{+}$monocytes was in the same levels as in the $\mathrm{CD} 14^{+}$ monocytes from MS patients (Figure 3.13.e), whereas it has been 2-fold lower in healthy subjects (Figure 3.12.e). This indicates an equal ability of $\mathrm{CD}^{+} 6^{+}$monocytes to migrate in the response to CCR2 ligand (CCL2 chemokine), as CD14 $4^{+}$monocytes in MS patients. 
a.

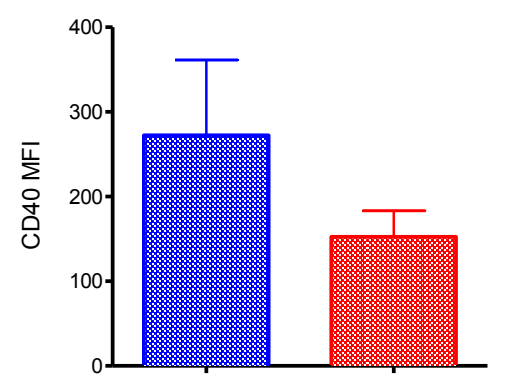

d.

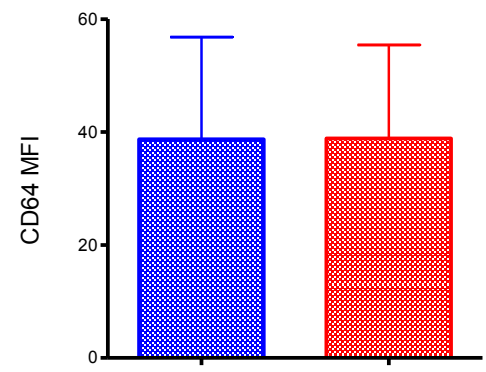

b.

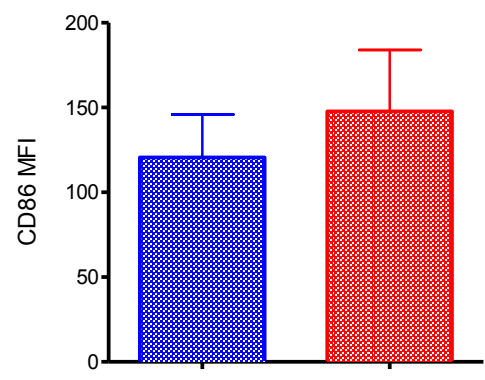

e.

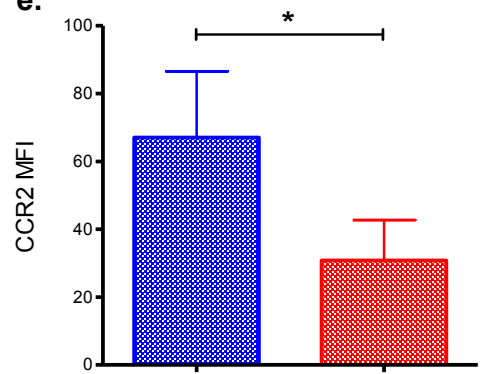

c.

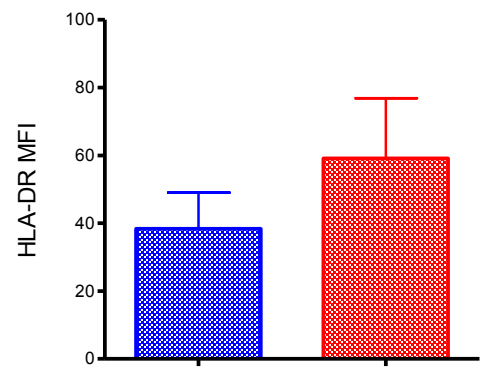

CD14+ mono

CD16+ mono

Figure 3.12. Healthy CD14 ${ }^{+}$monocytes had higher expression of CD40 and CCR2 and lower expression of CD86 and HLA-DR, than the CD16 ${ }^{+}$monocytes.

Total monocytes were isolated from PBMC using CD14 Microbeads. Isolated monocytes were stained with fluorescent flow antibodies and the expression was assessed by flow cytometry. CD $14^{++}$CD $16^{-}$CD $11 b^{++}$ monocytes were gated out from the total monocyte populations on flow cytometry data, based on the expression of CD11b, CD14 and CD16. (a) The expression of CD40 on subsets. (b) The expression of CD86 on subsets. (c) The expression of HLA-DR on subsets. (d) The expression of CD64 on subsets. (e) The expression of CCR2 on subsets. Shown are the means and SD of 20 individuals in healthy group. The statistical analysing was done by using Student's t-test to compare 2 groups. ${ }^{*} \mathrm{P}<0.01$. Data were collected on flow cytometry using the gating strategy in appendix 8. 
a.

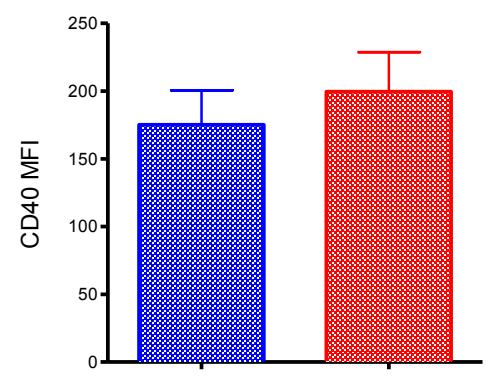

d.

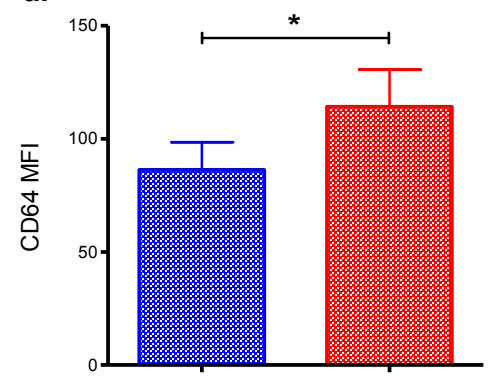

b.

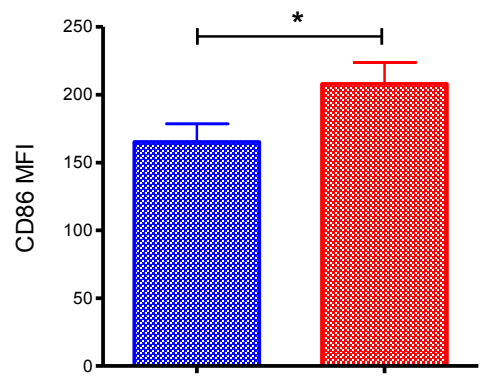

e.

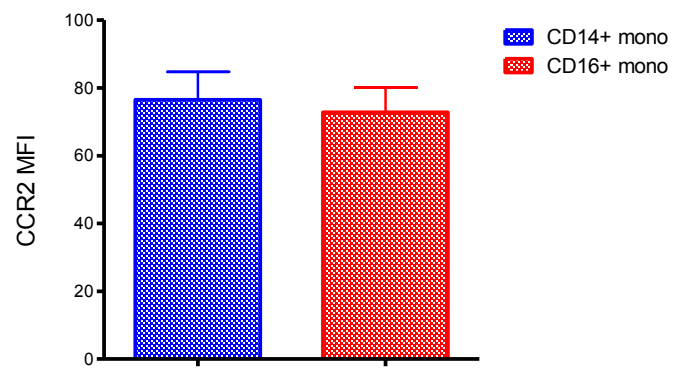

c.

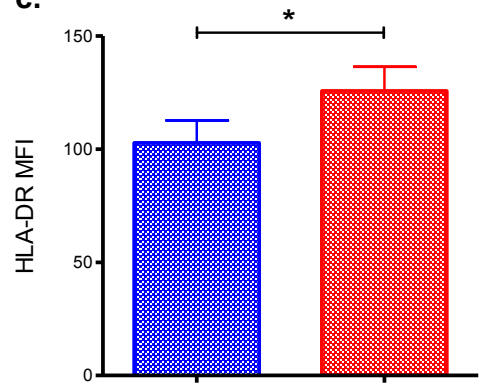

Figure 3.13. MS CD16 ${ }^{+}$monocytes had higher expression of activation markers than CD14 ${ }^{+}$monocytes.

Total monocytes were isolated from PBMC using CD14 Microbeads. Isolated monocytes were stained with fluorescent flow antibodies and the expression was assessed by flow cytometry. $\mathrm{CD} 14^{+} \mathrm{CD} 16^{+} \mathrm{CD} 11 \mathrm{~b}^{+}$ monocytes were gated out from the total monocyte populations on flow cytometry data, based on the expression of CD11b, CD14 and CD16. (a) The expression of CD40 on subsets. (b) The expression of CD86 on subsets. (c) The expression of HLA-DR on subsets. (d) The expression of CD64 on subsets. (e) The expression of CCR2 on subsets. Shown are the means and SD of 20 individuals in MS group. The statistical analysing was done by using Student's t-test to compare 2 groups. ${ }^{*} \mathrm{P}<0.01$. Data were collected on flow cytometry using the gating strategy in appendix 8.

\subsubsection{Summary}

In overall, monocytes from MS patients have characteristics of classically activated cells, when assessed ex vivo, whereas the monocytes from healthy subjects have only a basal state of activation. Among the monocyte subsets, $\mathrm{CD} 16^{+}$monocytes from MS patients have a more inflammatory phenotype than the $\mathrm{CD} 14^{+}$monocytes, which was different from the healthy subjects. These findings suggest that the monocytes of MS patients, especially the $\mathrm{CD} 6^{+}$subset, circulate in an inflammatory state that resembles classical activation. 


\subsection{DISCUSSION}

This chapter characterized the phenotype and activation of ex vivo isolated monocytes and their two main subsets in MS patients. These results showed that monocytes and their subsets could be identified in MS patients' blood by the same phenotypical features, as in healthy people; however, they had characteristics of inflammatory or classically activated monocytes. In contrast, the monocytes in healthy subjects had a basal state of activation.

\subsubsection{Characteristics of monocytes and their subsets}

Monocytes can be identified by a unique expression of cell surface markers, such as CD11b and CD14, whereas CD16 is an additional marker that distinguishes the CD16 ${ }^{+}$ monocyte subset from the $\mathrm{CD} 14^{+}$subset [34]. Supporting these observations, the results from our study showed that monocytes isolated from healthy and MS patients expressed both CD11b and CD14, although the level of expression varied between the different monocyte subsets. Depending on the degree of expression of these identifying markers, the CD14 ${ }^{+}$ monocytes can be further described as $\mathrm{CD} 11 \mathrm{~b}^{\text {high }} \mathrm{CD} 14^{\text {high }} \mathrm{CD} 16^{-}$cells, and the $\mathrm{CD} 16^{+}$ monocytes can be characterized as $\mathrm{CD} 11 \mathrm{~b}^{\text {low }} \mathrm{CD} 14^{\text {low }} \mathrm{CD} 16^{\text {med }}$ cells. This corresponds to the study by Ingersoll et al., which described the expression of CD14 and CD16 as the main phenotypical identification markers for the monocyte subsets in humans [41]. Additionally, this study indicated that the monocytes and their $\mathrm{CD}_{1} 4^{+}$and $\mathrm{CD} 16^{+}$subsets could be identified in the blood of MS patients by using the same hallmarks as in healthy subjects.

\subsubsection{Proportion of monocyte subsets in MS patients}

By using monocyte identification markers (i.e. CD11b, CD14 and CD16), the proportion of monocyte subsets were evaluated in MS patients. Although the percentage of monocytes remained within the normal range (11-16\% of PBMC), the proportion of CD14 $4^{+}$and $\mathrm{CD} 16^{+}$ monocytes was significantly altered in MS patients. Specifically, the proportion of CD14 monocytes was decreased and the proportion of $\mathrm{CD}^{+} 6^{+}$monocytes was significantly increased (i.e. by five-fold) in MS patients, in comparison to the healthy subjects. These results agree with a previous study, which reported a six-fold increase in the number of $\mathrm{CD} 16^{+}$monocytes ( $32 \%$ of total monocytes), and a subsequent decrease in CD14 ${ }^{+}$monocytes 
(68\% of total monocytes) in MS patients [97]. The subtle difference between this and others' studies may be due to variation in the methods used for the isolation of monocyte subsets. For example, Lopez-Moratalla et al. used an adherence method, which resulted in approximately 90\% pure monocytes. CD14 magnetic beads were used in this study, resulting in isolated monocytes with greater than $95 \%$ purity. The difference in the purity of isolated monocyte subsets can also affect the evaluation of the subset's ratio. Nethertheless, the results from our study are in line with observations reported in previous studies[97], as we showed similar changes in the proportion of monocyte subsets in MS patients.

The mechanism for the relative increase in the number of $\mathrm{CD} 16^{+}$monocytes in MS is unclear. However, it has been suggested that the $\mathrm{CD} 14^{+}$monocytes might differentiate into $\mathrm{CD}^{+} 6^{+}$monocytes [77], which could shift the profile of $\mathrm{CD}_{1} 4^{+}$monocytes into $\mathrm{CD} 16^{+}$ monocytes during the inflammation that occurs in MS. Therefore, the increased number of $\mathrm{CD} 6^{+}$monocytes in MS patients might be due to the replenishment of $\mathrm{CD}_{1} 6^{+}$monocytes from $\mathrm{CD} 14^{+}$monocytes, as the percentage of $\mathrm{CD} 14^{+}$monocytes drops down in parallel with the increase in number of $\mathrm{CD}^{+} 6^{+}$monocytes. Alternatively, it is also possible that the migration of monocytes to the site of inflammation results in a loss of the normal proportion of $\mathrm{CD} 14^{+}$and $\mathrm{CD} 16^{+}$subsets in the circulating blood of MS patients. CD16 $6^{+}$monocytes may play a greater role in the activation of inflammatory $\mathrm{T}$ cells compared to $\mathrm{CD} 14^{+}$monocytes. This imbalance could be an important factor in the dysregulation of T cells in MS, as the $\mathrm{CD}_{16}{ }^{+}$monocytes from MS patients exhibit a highly inflammatory phenotype.

\subsubsection{Expression of activation markers in monocytes and their subsets in MS}

In this study, the total monocyte population from MS patients displayed characteristics of classically activated (inflammatory) monocytes, as indicated by a higher expression of the activation markers CD40, CD86, HLA-DR, CD64 and CCR2, compared to monocytes isolated from healthy subjects. The total monocyte population also had the expanded population of $\mathrm{CD} 16^{+}$monocytes, which are believed to be the most inflammatory subset in MS patients. This study further compared the expression of activation markers between individual monocyte subsets from MS patients and healthy subjects, and characterized the inflammatory nature of these $\mathrm{CD} 14^{+}$and $\mathrm{CD} 16^{+}$monocytes. 
CD40 and CD86 are important molecules, and are co-stimulators of T cells. Blockade of this pathway prevents the development of MS in a mouse model [188-190]. In this study, the expression of CD40 and CD86 was significantly increased in the monocytes from MS patients, in comparison to healthy patients. This finding indicates that the inflammatory monocytes from MS patients are superior in their induction of $\mathrm{T}$ cell activation, compared to that of monocytes from healthy subjects. Interestingly, it seems that the high expression of CD40 and CD86 in MS patients was due to elevated numbers of CD16 ${ }^{+}$monocytes specifically. In MS patients, $\mathrm{CD} 16^{+}$monocytes have significantly higher levels of CD40 and CD86, compared to the $\mathrm{CD} 14^{+}$monocytes, suggesting a greater ability of $\mathrm{CD} 16^{+}$monocytes to co-stimulate T cells.

Both monocyte subsets from MS patients have a significantly higher expression of HLA-DR, compared to monocytes from the healthy subjects, supporting an idea of activation of inflammatory $\mathrm{T}$ cells through antigen presentation by the monocytes. HLA-DR is the main molecule that is responsible for the presentation of antigens to T cells by monocytes [191], and the increased level of HLA-DR has been associated with the exacerbation of MS[192]. When the expression was evaluated in the monocyte subsets from healthy people, the CD16 monocytes had a higher expression of HLA-DR, than the CD14 $4^{+}$monocytes. These results support a study by Passlick et al., which showed that in healthy patients, CD16 ${ }^{+}$monocytes had a two-fold higher level of HLA-DR, and might be more potent antigen presenting cells compared to $\mathrm{CD} 14^{+}$monocytes[85]. Our study showed, for the first time, that in MS patients, both $\mathrm{CD} 14^{+}$and $\mathrm{CD} 16^{+}$monocytes can be efficient antigen presenting cells. The two subsets had up-regulated expression of HLA-DR with only a very subtle difference (i.e. slightly higher in the $\mathrm{CD} 16^{+}$subset). This indicates that the $\mathrm{CD} 14^{+}$and $\mathrm{CD} 16^{+}$monocytes may both have increased antigen presenting ability in MS patients, but not healthy subjects.

The binding of CCR2 to its ligand (i.e. CCL2) induces the migration of monocytes to an inflammatory site [79] and is known to be involved in the disease progression in MS [68]. While CCL2 is expressed in neurons, microglia, astrocytes and brain endothelial cells[193], the expression levels of CCR2 were significantly increased in the total monocyte population from MS patients in our study. This suggests that in MS patients, there may be an increase in the migration of monocytes to the brain tissue due to inflammation in the CNS. A previous study showed that while this chemokine receptor was variably expressed on monocyte subsets in healthy people, the $\mathrm{CD} 14^{+}$monocytes had higher expression levels of CCR2, compared to the $\mathrm{CD}_{16}{ }^{+}$monocytes in healthy subjects [41], which is in agreement with the present study. In MS patients, however, the elevation in CCR2 was not due to the increase in 
$\mathrm{CD} 14^{+}$monocytes, but because of the significantly elevated expression in $\mathrm{CD} 16^{+}$monocytes. The CCR2 upregulation in $\mathrm{CD}_{1} 6^{+}$monocytes resulted in an equal final expression on $\mathrm{CD} 14^{+}$ and $\mathrm{CD}_{16}{ }^{+}$monocytes from MS patients, despite the high expression on the $\mathrm{CD} 14^{+}$ monocytes from healthy subjects. These results indicate that, in contrast to monocyte subsets from healthy subjects, both $\mathrm{CD}_{1} 4^{+}$and $\mathrm{CD} 16^{+}$monocytes from MS patients can be efficiently stimulated to migrate to the inflammatory site in the response to the chemokine, CCL2.

The binding of CD64, a high affinity Fc $\gamma$ receptor I, induces inflammation and the clearance of pathogens from the organism [194]. The expression of CD64 in monocytes from healthy patients was similar in both subsets. These results are contradictory to other studies, which have observed a higher level of CD64 in CD14 ${ }^{+}$monocytes in healthy people [41]. This discrepancy might be due to differences in the cell type used by Ingersoll et al., where they examined a complex cell population (i.e. PBMC) consisting of monocytes, lymphocytes and some granulocytes. In this study, a purified monocyte population was used.

Monocytes, residing in the brain of MS patients (macrophages) have high expression levels of CD64, which is indicative of phagocytic activation of the monocytes [195]. In parallel, this study found that the circulating blood monocytes in MS patients also had induced expression of CD64, and that the $\mathrm{CD}^{+} 6^{+}$monocytes had a higher expression than the $\mathrm{CD} 14^{+}$monocytes. Differentiated $\mathrm{CD}^{+} 4^{++} \mathrm{iNOS}^{++}$monocytes have been found in active lesions of the CNS in MS patients, and these macrophages contained intracellular myelin fragments indicating the phagocytosis of myelin debris and the possible direct damage of the myelin sheath by these cells [195]. CD16 ${ }^{+}$monocytes are the main producers of iNOS [97], which is an enzyme that synthesizes NO, a product known to be directly involved in the nerve damage in MS [196]. The $\mathrm{CD}^{++}{ }^{+} \mathrm{iNOS}^{++}$cells are highly likely to be derived from the $\mathrm{CD}_{16}{ }^{+}$monocytes. In agreement with this, Hill et al. have shown that the inflammatory $\mathrm{CD}^{+++}$iNOS $^{++}$cells are not $\mathrm{CD} 14^{+}$monocytes, as the $\mathrm{CD}_{14}{ }^{+} \mathrm{iNOS}^{\text {low }}$ macrophages have been found in the surrounding tissue, not in the active inflammatory sites. This finding suggests that $\mathrm{CD} 14^{+}$monocytes play the role of scavenger cells, whose function is to clear post-inflammatory debris and apoptotic cells in MS [195]. Taken together, these observations indicate that the $\mathrm{CD}_{1} 6^{+}$monocytes are the main cells that are responsible for the phagocytosis and direct damage of nerve proteins in MS.

To conclude, the enhanced expression of activation markers showed a highly inflammatory nature, similar to the classical activation state of the monocytes in MS patients. Although the ability to phagocytose, migrate and present antigens appear to be similar in both 
monocyte subsets, the $\mathrm{CD} 16^{+}$monocytes might be more effective in the co-stimulation of $\mathrm{T}$ cells, in addition to causing direct damage to the brain tissue in MS. This study has characterized for the first time the activation of monocytes and their subsets during MS, in a New Zealand population. 


\section{Chapter 4.}

\section{Type II activation of total monocytes in vitro}




\subsection{INTRODUCTION}

Monocytes can play a dual role in MS by promoting or resolving inflammation. Classically activated monocytes contribute to inflammation in MS by directly damaging oligodendrocytes and nerve cells[73], and activating other immune cells, including Th1 and Th17 cells[24]. Monocytes activate T cells through production of inflammatory cytokines[69, 70], co-stimulatory molecules such as CD40[71] and CD86[52], and antigen presentation by HLA-DR molecules[31]. However, if type II activated, monocytes may also play a regulatory role. While classically activated monocytes induce inflammation in MS, it has been shown that type II activated macrophages can ameliorate disease in a mouse model of MS[44]. Unlike classically activated monocytes, type II activated monocytes produce low levels of the inflammatory cytokines and activation markers and a high amount of anti-inflammatory cytokine IL-10[21]. Thus, through the action of IL-10 and other anti-inflammatory factors, type II activation of monocytes is a beneficial approach in the treatment of MS.

Recently, it has been shown that glatiramer acetate (GA) has a direct type II activating effect on monocytes in a mouse model of MS, and GA induces activation of antiinflammatory Th2 cells[43] and inhibits the proliferation of inflammatory T cell subsets[166]. In human studies, GA inhibits the production of inflammatory molecules SLAM (signaling leukocyte activation molecule) and TNF $\alpha$ that are involved in activation of $\mathrm{T}$ cells[32], possibly as a result of the type II activation of human monocytes, yet the mechanism of action of GA is not well understood. Although it is clear that monocytes are important mediators of GA effect in MS, its direct type II activating effect on human monocytes has not been fully characterized. Therefore, this study aims to elucidate the direct type II activating effect of GA treatment on human monocytes in MS.

Immune complexes (IC) have also been shown to have a type II activating effect on differentiated monocytes (i.e. macrophages) and ameliorate disease in a mouse model of MS[44]. Although IC can directly affect human monocytes[178], the ability of IC to type II activate human monocytes has not been investigated. Hence, it is important to understand the direct effect of IC on human monocytes and characterize its type II activating effect. 


\subsection{AIMS}

It was hypothesized that GA and IC can type II activate human monocytes in MS. In order to examine this hypothesis, the following aims have been proposed in this chapter:

1. To compare the classical activation of monocytes from both healthy subjects and MS patients.

2. To characterize the type II activation of monocytes by GA and IC in healthy subjects and MS patients. 


\subsection{RESULTS}

\subsubsection{Optimization of monocyte culture and treatments}

\subsubsection{Optimization of classical activation}

Classical activation of monocytes was induced by IFN- $\gamma$ priming for 16 hours followed by stimulation with LPS. Both IFN- $\gamma$ and LPS were shown to be essential for the classical activation, as cytokine production was not detected in the absence of one of these stimulants. IFN- $\gamma$ was used at a concentration of $20 \mathrm{U} / \mathrm{ml}$ since this was shown to be an optimal dose for the priming of monocytes. At this concentration IFN- $\gamma$ had a similar effect at inducing cytokines as at higher concentrations and induced a good response to LPS in monocytes. In both low and high concentrations of IFN- $\gamma$, LPS stimulation was required for IL-12 production since cytokine production was not observed in the absence of LPS (Figure 4.1.a).

Two different concentrations of LPS were tested for the stimulation of monocytes and induction of IL-12 production: $100 \mathrm{ng} / \mathrm{ml}$ and $200 \mathrm{ng} / \mathrm{ml}$. The effect of these LPS concentrations on monocyte viability was investigated using the colorimetric MTT assay. In this study, stimulation of monocytes with LPS at concentrations of $100 \mathrm{ng} / \mathrm{ml}$ and $200 \mathrm{ng} / \mathrm{ml}$, did not have a cytotoxic effect since no change was detected in the MTT response. This finding suggests that LPS at both concentrations is not toxic to monocytes (Figure 4.1.b). It was found that $200 \mathrm{ng} / \mathrm{ml}$ LPS was more potent at inducing cytokine production in monocytes, indicating efficient activation of monocytes by LPS at this concentration (Figure

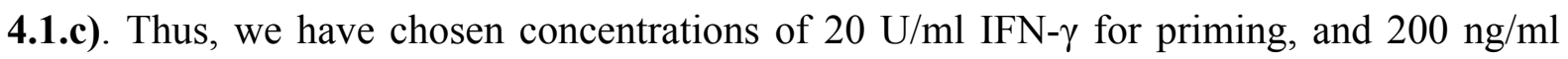
LPS for stimulation as optimal conditions for the activation of monocytes.

To find an optimal duration of stimulation, monocytes were incubated with either LPS or no LPS for 2, 4, 10 and 24 hours after IFN- $\gamma$ priming. As expected, in the absence of LPS no IL-12 production was detected at any of the time points. The highest induction of IL-12 production was observed after stimulation with LPS for 24 hours, in comparison to medium only (Figure 4.1.d). Thus, stimulation with LPS for 24 hours was chosen as an optimal time to assess the classical activation of monocytes. 
a.

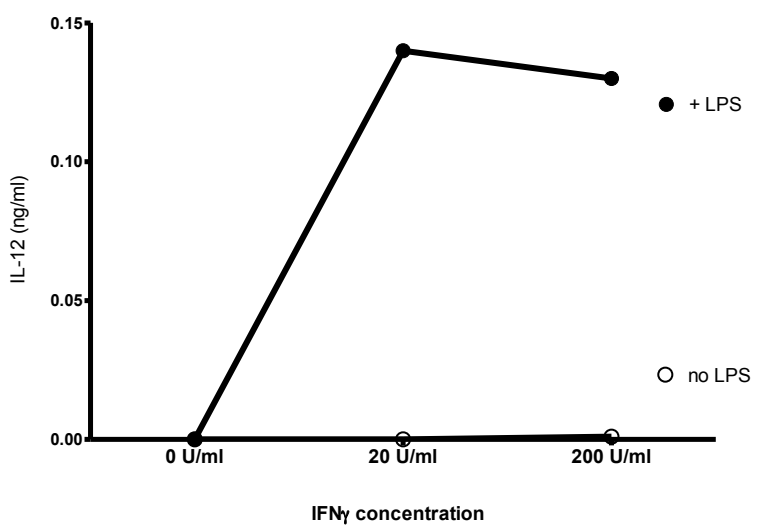

c.

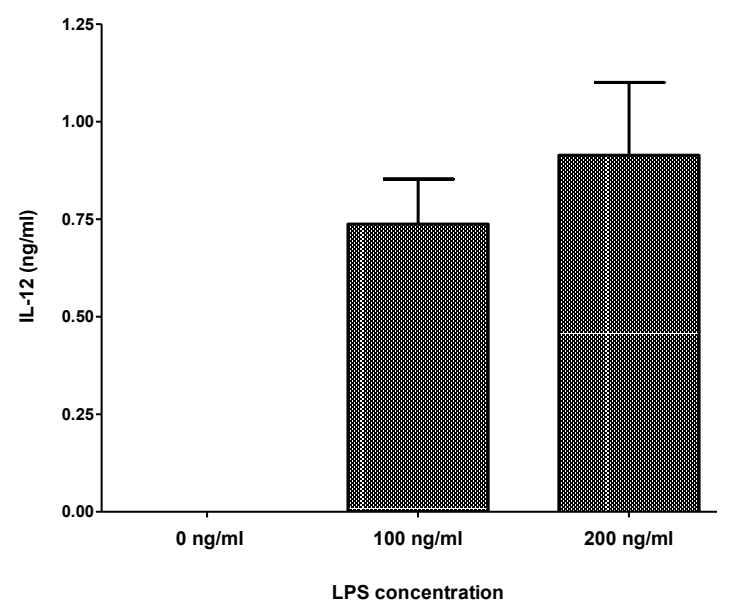

b.

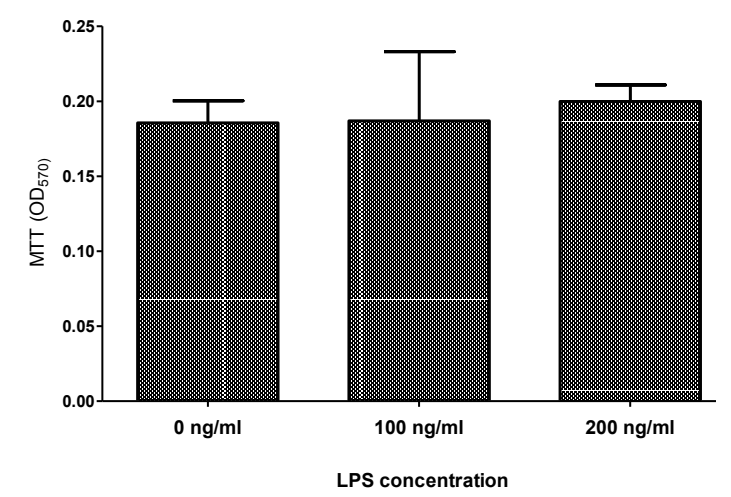

d.

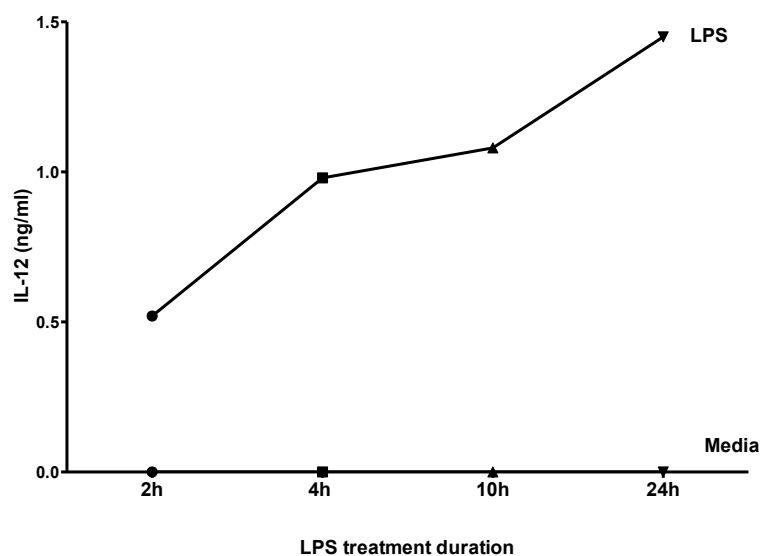

Figure 4.1. IFN- $\gamma$ priming at $20 \mathrm{U} / \mathrm{ml}$ (a) and LPS stimulation at $200 \mathrm{ng} / \mathrm{ml}$ (c) promotes the best classical activation (i.e. IL-12p40 production) 24 hours (d) post priming.

(a) Total monocytes were isolated from healthy subjects and primed with various doses of IFN- $\gamma$ for 16 hours before culturing in the presence or absence of LPS. (b) After 24 hours, the effect of LPS on monocyte viability was assessed by the MTT assay and expressed as optical density $\left(\mathrm{OD}_{570}\right)$. (c) IL-12 production in the supernatants was measured by ELISA. (d) IL-12 production by IFN- $\gamma$ primed $(20 \mathrm{U} / \mathrm{ml})$ monocytes cultured in the presence or absence of LPS $(200 \mathrm{ng} / \mathrm{ml})$ was assessed at various times post priming by ELISA. Shown are the means and SD of triplicate wells from three different experiments from three different individuals. 


\subsubsection{Optimization of GA treatment}

In order to ensure that GA had no adverse effects on monocyte viability, an MTT assay was performed. Monocytes were treated with or without LPS in the presence of GA at two different concentrations. Treatment with $200 \mu \mathrm{g} / \mathrm{ml}$ GA, regardless of LPS concentration, decreased monocyte viability. However, at a concentration of $100 \mu \mathrm{g} / \mathrm{ml} \mathrm{GA}$ had no cytotoxic effect on monocytes as the OD was similar to that of in untreated cells (Figure 4.2.a), which suggests that treatment of monocytes with $100 \mu \mathrm{g} / \mathrm{ml}$ of GA is an optimal concentration for the treatment.

In order to test if GA alone activates monocytes, a no-LPS control was used to assess activation. GA treatment of monocytes without LPS stimulation did not induce IL-12 production (Figure 4.2.b). LPS stimulation is thus a requirement for the monocytes to produce IL-12 (Figure 4.2.b), indicating that activation of monocytes is due to the LPS and not the GA.

a.

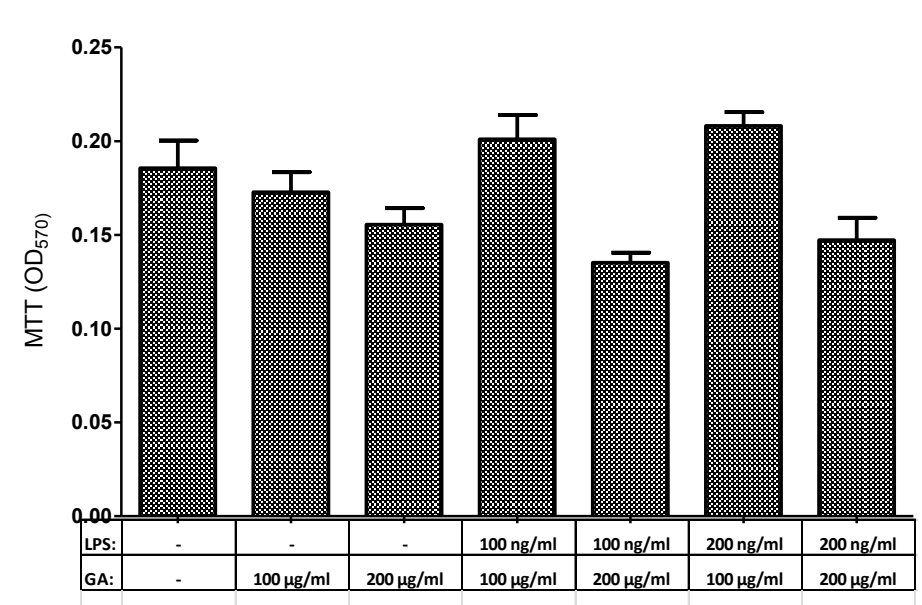

b.

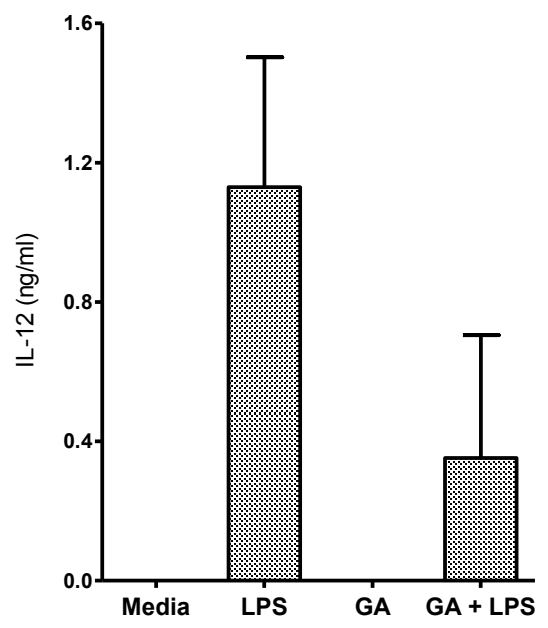

Figure 4.2. Optimization of GA treatment of monocytes.

Total monocytes were isolated from healthy subjects and primed with $20 \mathrm{U} / \mathrm{ml}$ IFN- $\gamma$ for 16 hours. Monocytes were cultured with GA in the presence or absence of LPS for 24 hours. (a) The effect of GA on monocyte viability was assessed by the MTT assay and expressed as optical density $\left(\mathrm{OD}_{570}\right)$. (b) IL-12 production in the supernatants was measured by ELISA. Shown are the means and SD of triplicate wells from three different experiments from three different individuals. 


\subsubsection{Optimization of IC treatment}

Previous studies using IC to induce type II activation have used a variety of different IC, such as opsonized sheep red blood cells (RBC) or IgG-coated ovalbumin[173, 197]. Because IVIG contains antibodies to blood group antigens, such as A and B[174, 175], IVIG was used to coat human RBC to create large IC in the present study. Additionally, this IC may also occur naturally when $\mathrm{A}, \mathrm{B}$, or $\mathrm{AB}$ positive patients are treated with IVIG and thus, may represent a natural induction of type II activation. However, before using IC, a specificity and optimal dose were first investigated.

To confirm IC formation, specificity of Ab (IVIG) binding to RBC was examined by using PECy5-conjugated goat anti-human Ig to detect the antibody-coated RBC by flow cytometry. It was found that antibodies specifically bound to RBC at a number of different IVIG dilutions, and a 1:100 dilution of IVIG showed a good binding of the antibody to RBC (Figure 4.3.a). Thus, the 1:100 dilution of IVIG was chosen as an optimal dose for the IC treatment of monocytes. To ensure that IC treatment did not impair the viability of monocytes, two different dilutions of IVIG (1:100 and 1:200) were tested by MTT assay. IC at both dilutions of IVIG did not alter the results of MTT assay, indicating on no cytotoxic effect of IC on monocytes (Figure 4.3.b).

Since type II activation by IC requires Fc $\gamma \mathrm{R}$ ligation in the presence of an inflammatory stimulus such as LPS, the ability of IC alone to activate monocytes was investigated. Monocytes treated with IC in the absence of LPS did not produce any IL-12, indicating on the lack of activation of the monocytes upon only IC treatment. In contrast, the monocytes, treated with LPS in the presence or absence of IC, produced high concentrations of IL-12 (Figure 4.3.c), indicating that activation of monocytes is specifically dependent on LPS, but not on the IC.

To assess any non-specific effect of RBC on monocytes, unopsonized RBC (i.e. RBC, not coated with IVIG) were used. While IC treatment in the presence of LPS slightly inhibited IL-12 production of monocytes compare to LPS alone, unopsonized RBC treatment did not have any inhibitory effect on monocytes (Figure 4.3.d). This finding indicates that the IC effect on monocytes is not due to RBC alone, but is dependent on the IVIG bound to the RBC.

As a component of IC, IVIG exhibits its effect by binding to A antigens on RBC from blood group A, RBC (A), and also to B antigens from blood group B[198]. In order to confirm this, RBC from blood group $0, \mathrm{RBC}(0)$, that lack $\mathrm{A}$ and $\mathrm{B}$ antigens were used as a 
mismatched negative control. To test if the IVIG effect on monocytes is dependent on the presence of the A antigen on $\mathrm{RBC}$, monocytes were treated with either $\mathrm{RBC}(\mathrm{A})$, or $\mathrm{RBC}(0)$, opsonised with IVIG. The IC effect was assessed by its effect on IL-12 production in LPSstimulated monocytes. As such, when monocytes were treated with opsonised RBC (A), the production of IL-12 was decreased, in comparison to classically activated monocytes. This indicates that opsonised RBC (A) has an effect on monocytes. However, the monocytes, treated with opsonised RBC (0), did not show any alteration in the production of IL-12, indicating that the IVIG effect on monocytes is dependent on the presence of A antigen on RBC (Figure 4.3.e). Therefore, RBC (A) were used to make IC in the present study.

Overall, the following optimal conditions were chosen for in vitro stimulations and treatments of monocytes in this study: $20 \mathrm{U} / \mathrm{ml}$ of IFN- $\gamma$ priming, $200 \mathrm{ng} / \mathrm{ml}$ of LPS, 100 $\mu \mathrm{g} / \mathrm{ml}$ of GA and 1:100 dilution of IVIG and 10 RBC per monocyte for IC treatment. A 16hour IFN- $\gamma$ priming was followed by 24 -hour treatment with LPS, in the presence or absence of GA or IC. 
a.

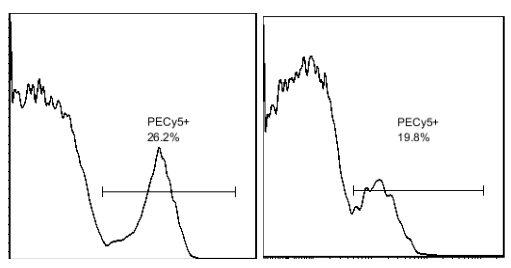

$1: 5$

$1: 20$

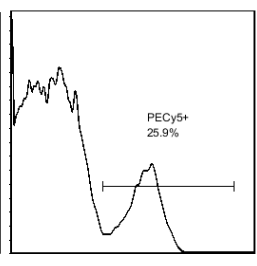

$1: 50$

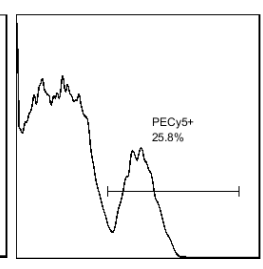

$1: 100$

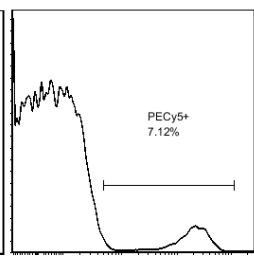

$1: 200$

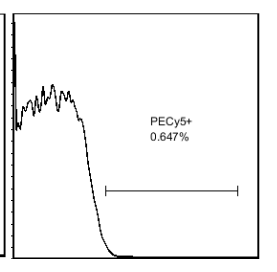

no IVIG b.

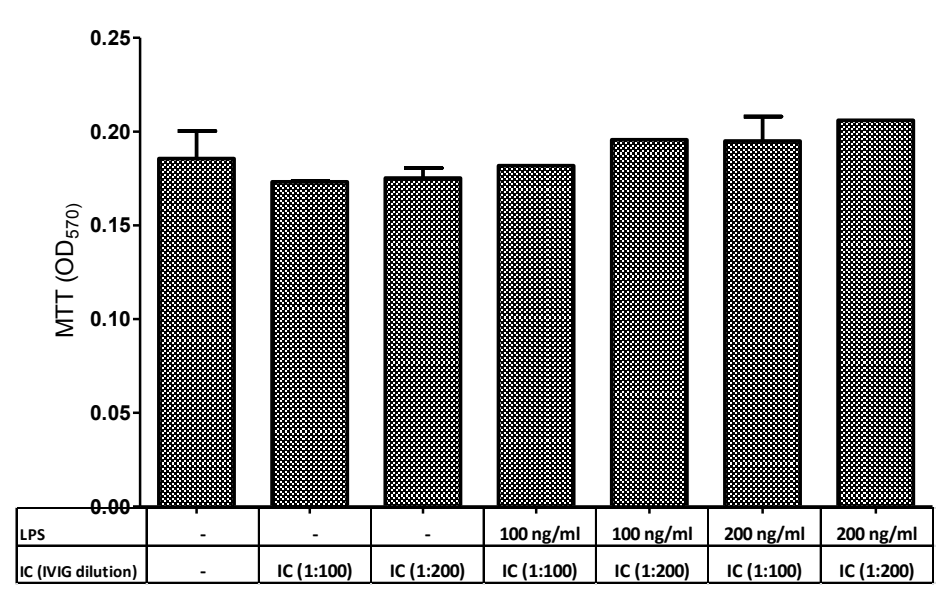

c.

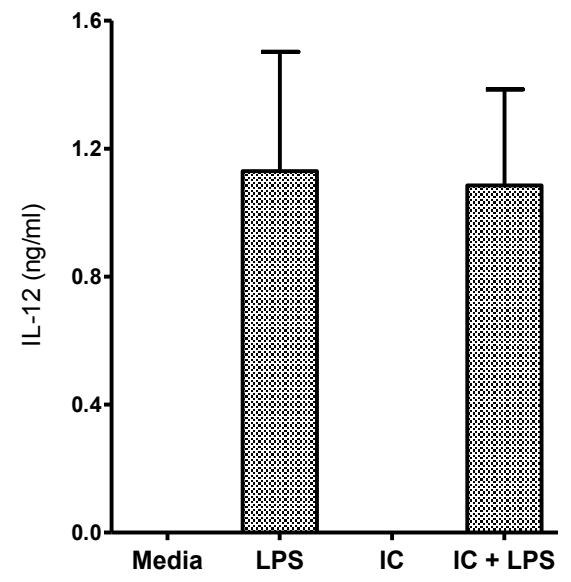

d.

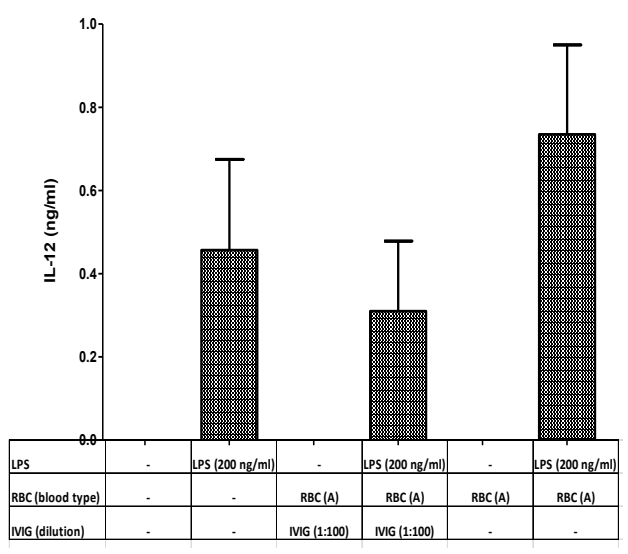

e.

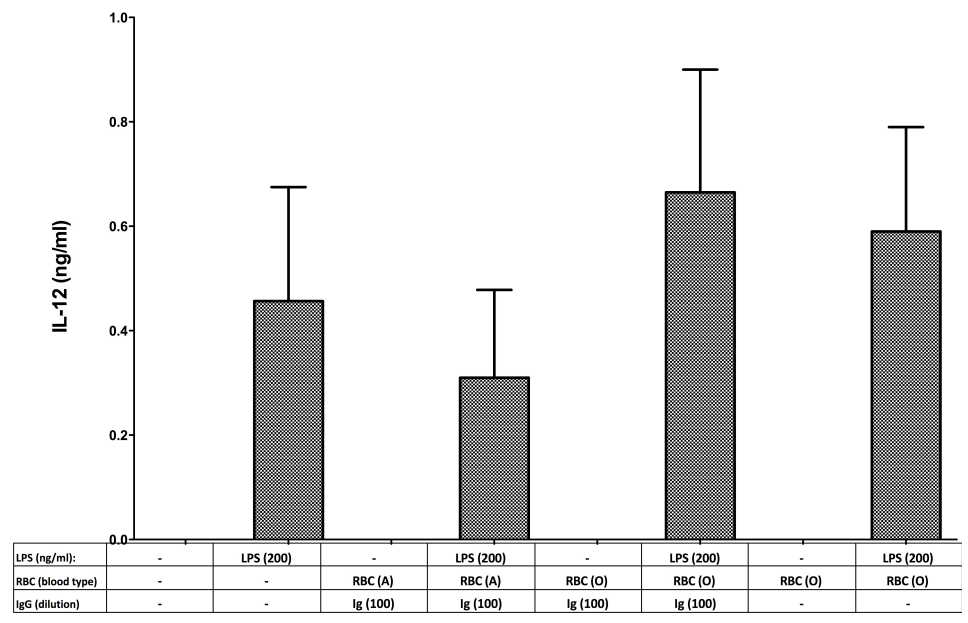

Figure 4.3. Optimization of IC treatment of monocytes.

(a) Human RBC were incubated with various dilutions of IVIG for $30 \mathrm{~min}$ in room temperature to allow the formation of IC and stained with PECy5-conjugated goat anti-human Ig. The PECy5 - conjugated IC were detected by flow cytometry. (b-d) Total monocytes were isolated from healthy subjects, primed with $20 \mathrm{U} / \mathrm{ml}$ IFN- $\gamma$ for 16 hours. Monocytes were cultured with IC in the presence or absence of LPS for 24 hours. (b) The effect of IC on monocyte viability was assessed by the MTT assay and expressed as optical density $\left(\mathrm{OD}_{570}\right)$. $(\mathrm{c})$ IL-12 production in the supernatants was measured by ELISA. (d) Monocytes were cultured with IC or RBC in the presence or absence of LPS. (e) Monocytes were treated with either RBC (A), or RBC (0), opsonized with IVIG in vitro. Shown are the means and SD of triplicate wells from three different experiments from three different individuals. 


\subsubsection{Classical activation of monocytes}

To induce classical activation, the isolated total monocyte populations were primed with IFN- $\gamma$ overnight and stimulated with LPS for 24 hours. In comparison to untreated monocytes, LPS-treated monocytes had significantly higher expression of CD40, and nonsignificantly higher expression of CD86 and HLA-DR in both healthy and MS groups (Figure 4.4). No cytokines were detected in supernatants from unstimulated monocytes from either group. However, when treated with $200 \mathrm{ng} / \mathrm{ml}$ LPS in vitro, both healthy and MS monocytes produced very high amounts of IL-6 and IL-12 and some IL-10, a pattern indicative of classical activation. Interestingly, the classically activated MS monocytes were, in general, more active in the production of cytokines and cell surface markers (Figure 4.4). As such, they expressed significantly higher level of CD86 and non-significantly higher level of CD40 and HLA-DR, and also produced greater amounts of IL-6 compared to the classically activated healthy monocytes. These results indicate that both healthy and MS monocytes can be classically activated and have a similar pattern albeit a different level of cytokine and activation marker expression. These LPS-treated, classically activated monocytes can thus be used as a model of inflammatory cells (a positive control) to further assess the type II activating effects of IC and GA treatments. 

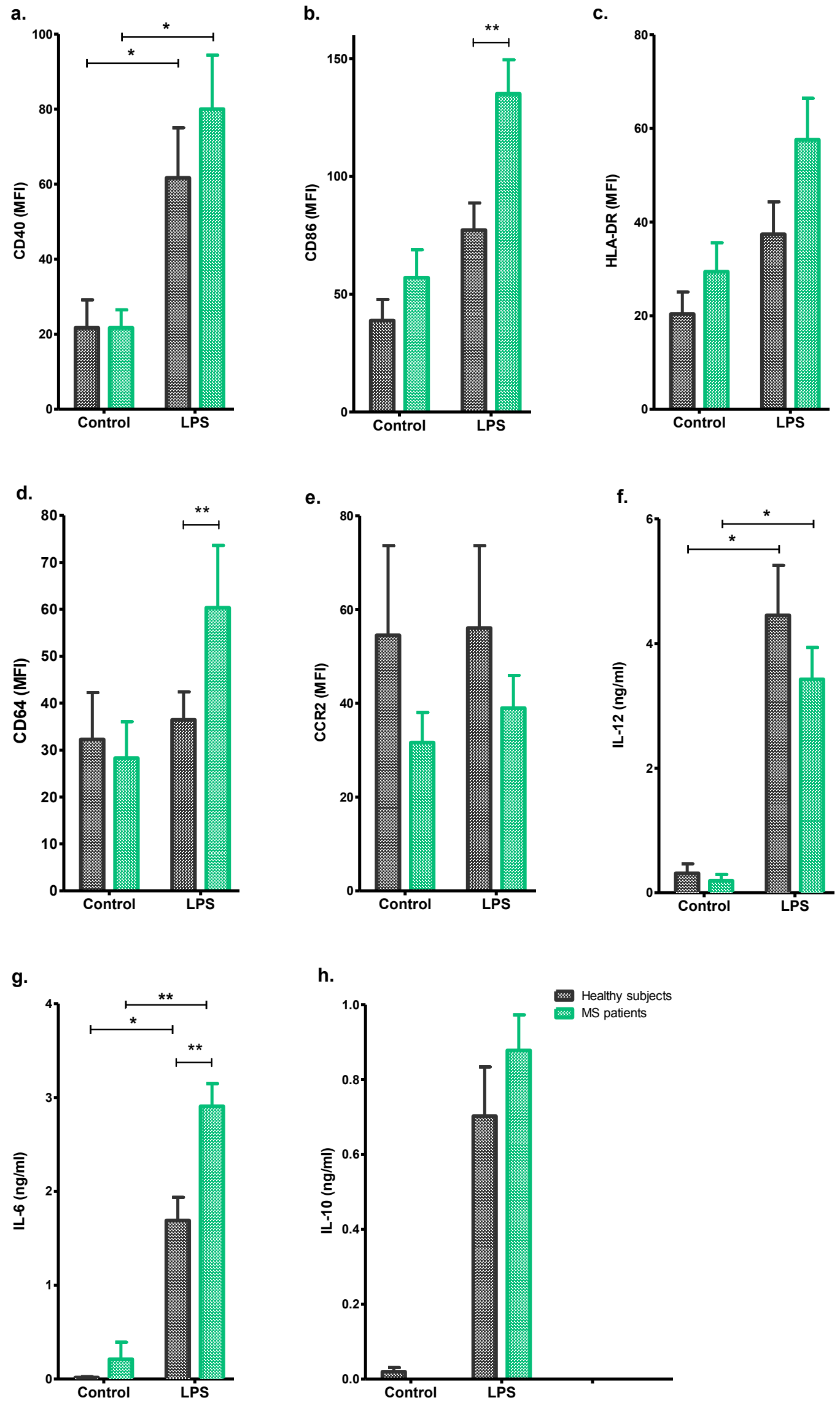
Figure 4.4. LPS stimulation induces classical activation of monocytes from healthy subjects and MS patients in vitro.

Total monocytes from healthy subjects $(n=23)$ and MS patients $(n=20)$ were primed with 20 U/ml IFN- $\gamma$ overnight and stimulated with $200 \mathrm{ng} / \mathrm{ml}$ LPS for 24 hours. The monocytes were then harvested and stained with antibodies. The activation marker expression was assessed by flow cytometry and the cytokine production was measured by ELISA. (a) Expression of CD40 on monocytes. (b) Expression of CD86 on monocytes. (c) Expression of HLA-DR on monocytes. (d) Expression of CD64 on monocytes. (e) Expression of CCR2 on monocytes. (f) Production of IL-12 in monocytes. (g) Production of IL-6 in monocytes. (h) Production of IL-10 in monocytes. Shown are the means and SD of duplicate wells from individual experiments in healthy and MS groups. The statistical analysing was done by using Student's t-test to compare 2 groups. $* \mathrm{P}<0.01 ; * * \mathrm{P}<0.005$. Data were collected on flow cytometry using the gating strategy in appendix 9 . 


\subsubsection{GA effect on monocytes in vitro}

GA treatment of monocytes from healthy subjects, in the presence of LPS, resulted in a significant downregulation of CD40, CD86 and IL-12 levels compared to LPS alone (Figures 4.5.a,b,d). There was a slight decrease in the expression of HLA-DR (Figure 4.5.c), and in parallel, the extracellular level of IL-10 was increased by $34 \%$, in comparison to the classically activated monocytes (Figure 4.5.g). Similarly, MS patients' monocytes showed a phenotype of type II activation after GA treatment. Although levels of HLA-DR and IL-10 did not alter after the treatment, monocytes had significantly less CD40, CD86 and IL-12 than classically activated monocytes from MS patients (Figures 4.5.a,b,d) and a slightly decreased production of IL-6 (Figure 4.5.e). This suggests that monocytes from healthy subjects and MS patients can be type II activated by in vitro GA treatment.

\subsubsection{IC effect on monocytes in vitro}

When monocytes from healthy subjects were treated with IC in the presence of LPS, there was no change in level of CD86 and only a minimal inhibition of production of IL-6 and IL-12 (Figures 4.5.d,e). In contrast the expression of CD40 and HLA-DR was significantly reduced (Figures 4.5.a,c). Moreover, production of the anti-inflammatory cytokine IL-10 was increased by $36 \%$ after IC treatment, in comparison to classically activated monocytes (Figure 4.5.g).

The effect of IC on MS patients' monocytes was slightly different from that of healthy subjects'. Instead of affecting HLA-DR expression, as observed in the healthy group, IC downregulated the expression of CD86 although there was a substantial variability between the individual patients and the decrease was not significant (Figure 4.5.b,c). As in healthy monocytes, IC significantly reduced CD40 on monocytes from MS patients (Figure 4.5.a). Although IL-6 and IL-12 production was not changed after IC treatment in MS patients (Figures 4.5.d,e), production of IL-10 showed a trend to increase (by 15\%) compared to classically activated monocytes (Figure 4.5.g). These results suggest that in vitro IC treatment can type II activate monocytes in both healthy and MS groups, although the degree of activation is lower in MS patients. 


\subsubsection{The effect of GA and IC combination treatment on monocytes in vitro}

Whereas GA and IC individual treatments can type II activate monocytes, it is not clear how a combination of those two treatments affects monocyte activation. To examine this, monocytes were treated with both GA and IC in the presence of LPS for 24 hours. In healthy subjects, a combination of IC and GA treatments was more effective in type II activation of monocytes than type II activation by IC or GA alone. As such, the expression of CD40, HLA-DR and IL-12 was significantly inhibited by the combination treatment (Figures 4.5.a,c,d), which not only suppressed the production of inflammatory molecules, but also enhanced the production of regulatory molecules like IL-10. Thus, the production of IL-10 was increased by $64 \%$, in comparison to classically activated monocytes in healthy subjects (Figure 4.5.g).

In MS group, monocytes had significantly reduced levels of CD40, CD86 and IL-12 after the combination treatment (Figures 4.5.a,b,d). In comparison to single treatments, the combination treatment resulted in a larger decrease in IL-12 production. However, unlike the healthy group, the combination treatment of monocytes in MS group only inhibited their inflammatory phenotype and did not increase anti-inflammatory factors. As such, the decrease in HLA-DR and the increase in IL-10 production were not significant in the patients' monocytes after the combination treatment (Figures 4.5.c,f). Overall, the combined treatment of monocytes from healthy subjects with IC and GA had a better effect than either treatment alone, and a similar effect was observed in monocytes from MS patients. Thus, monocytes from both healthy and MS groups showed the characteristics of type II activation after IC and GA combination treatment. 
a.

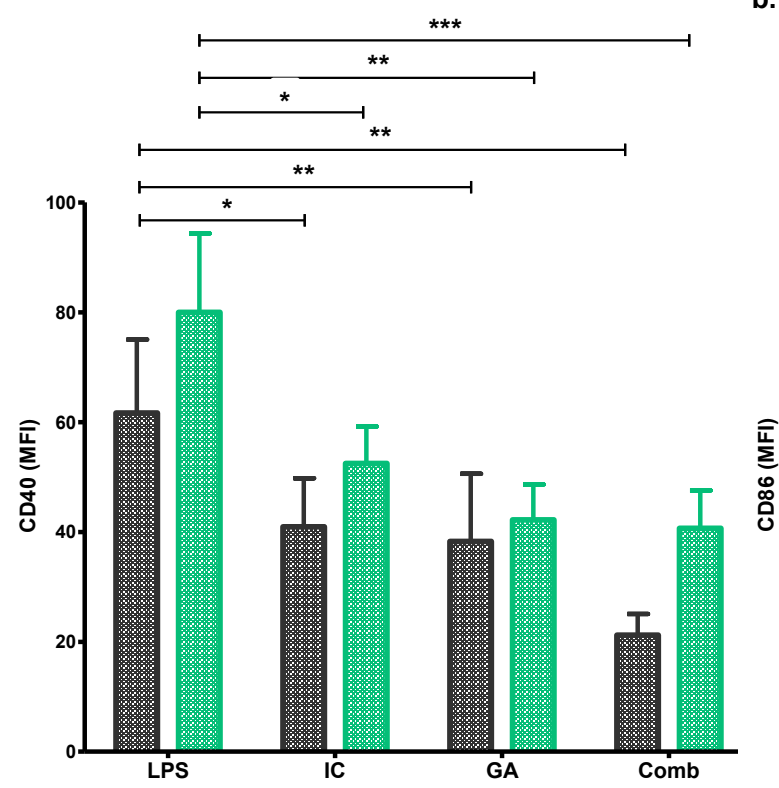

c.

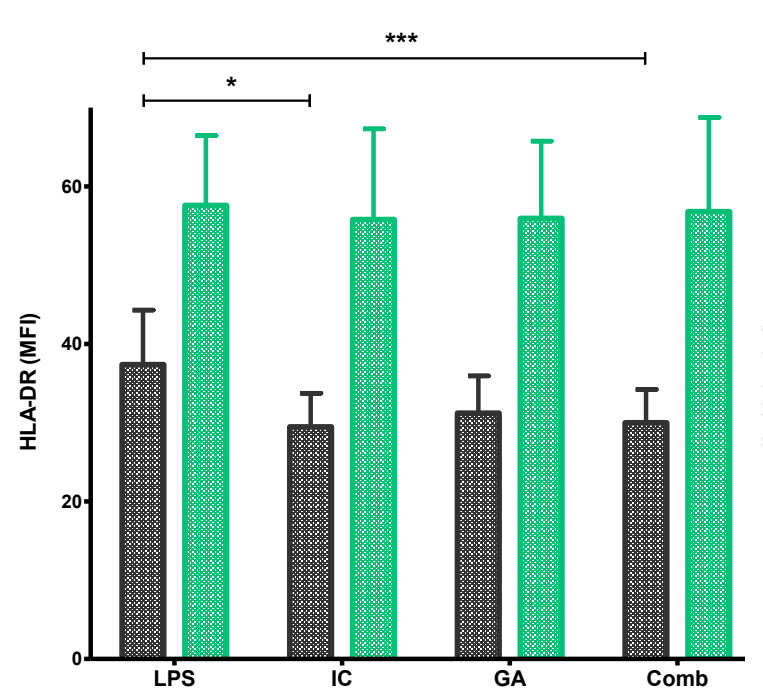

b.

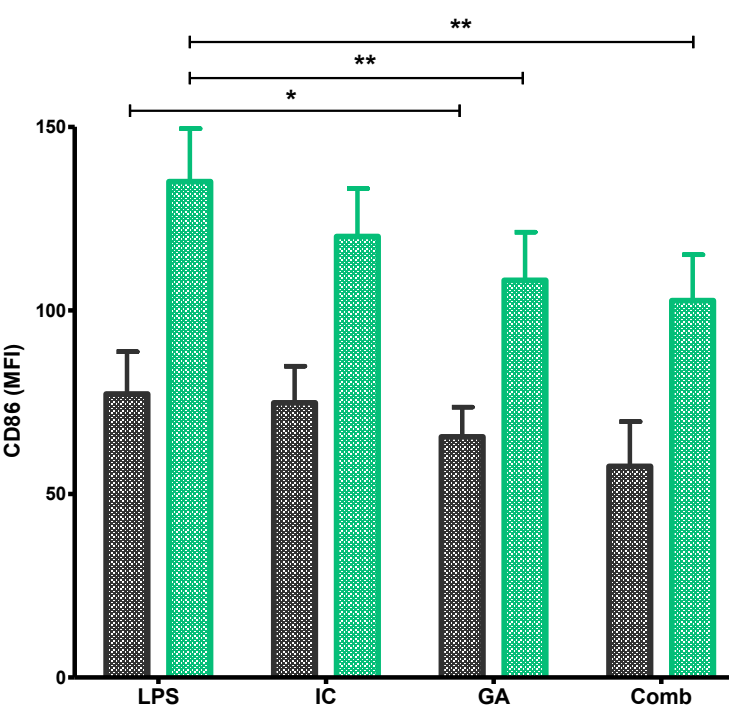

d.

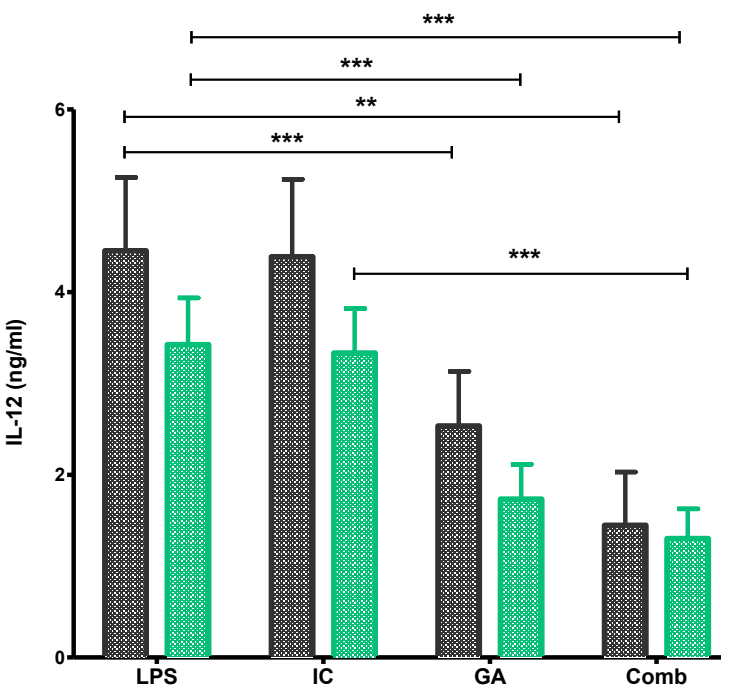

e.

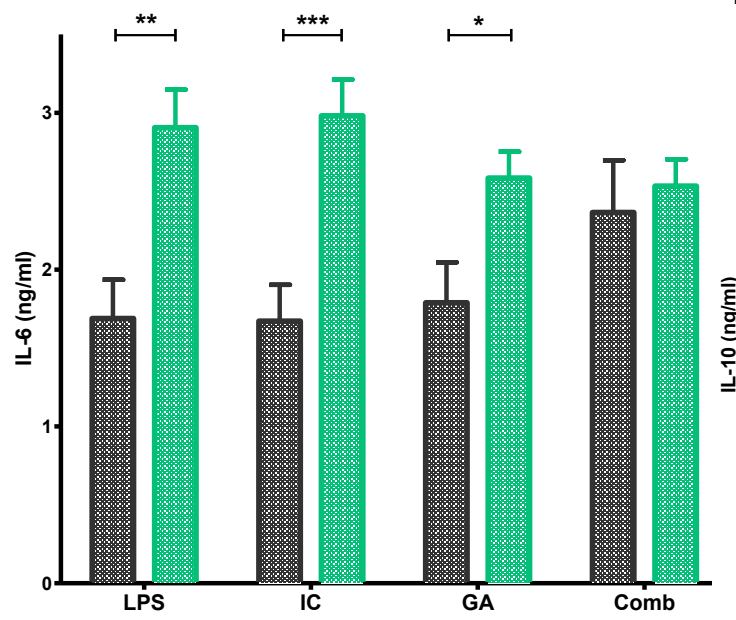

f.

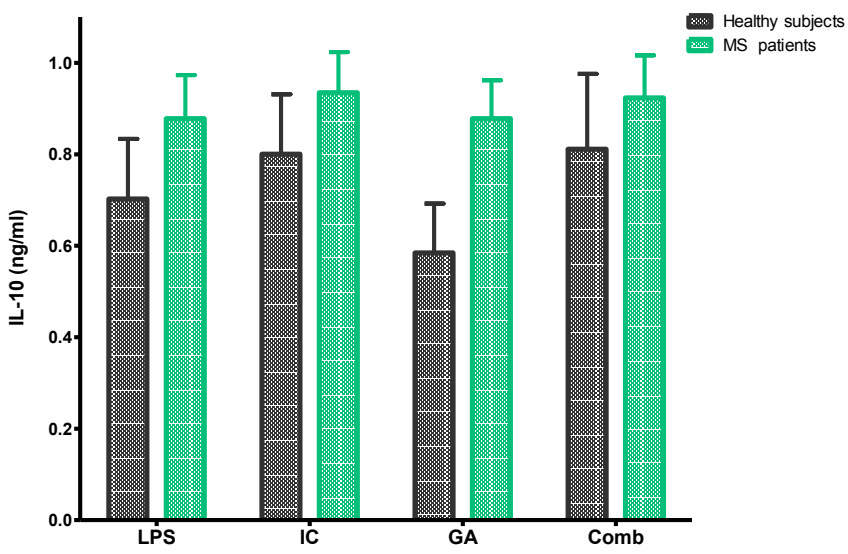




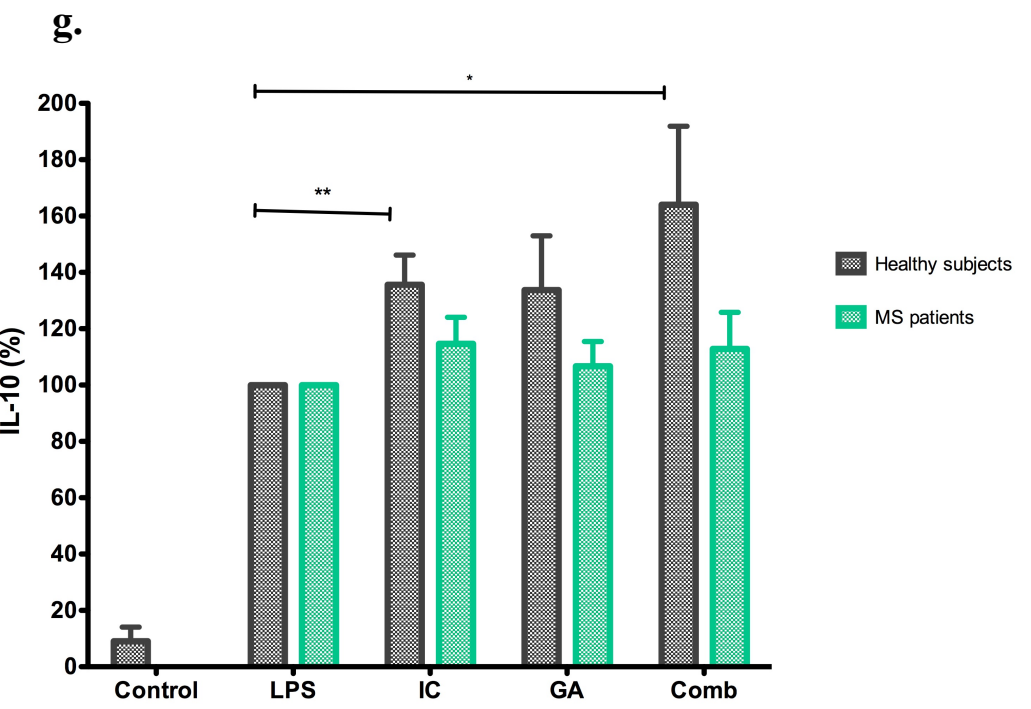

Figure 4.5. Type II activation of monocytes by GA and IC in vitro.

Total monocytes from healthy subjects $(n=23)$ and MS patients $(n=20)$ were primed with $20 \mathrm{U} / \mathrm{ml}$ IFN- $\gamma$ overnight and treated with $100 \mu \mathrm{g} / \mathrm{ml} \mathrm{GA}$ and/or IC in the presence or absence of $200 \mathrm{ng} / \mathrm{ml} \mathrm{LPS} \mathrm{for} 24$ hours. The monocytes were then harvested and stained with antibodies. The activation marker expression was assessed by flow cytometry and the cytokine production was measured by ELISA. (a) Expression of CD40 on monocytes. (b) Expression of CD86 on monocytes. (c) Expression of HLA-DR on monocytes. (d) Production of IL-12 in monocytes. (e) Production of IL-6 in monocytes. (f) Production of IL-10 in monocytes. (g) Production of IL-10 shown as a relative percentage to LPS. LPS group was normalized as $100 \%$. Shown are the means and SD of duplicate wells from individual experiments in healthy and MS groups. The statistical analysing was done by using one way ANOVA with Dunnett's post test to compare three or more groups and Student's t-test to compare 2 groups. $* \mathrm{P}<0.01 ; * * \mathrm{P}<0.005 ; * * * \mathrm{P}<0.001$. Data were collected on flow cytometry using the gating strategy in appendix 9. 


\subsubsection{Detection of intracellular cytokines}

Previously in this chapter we have looked at how LPS, GA and IC alter extracellular cytokine secretion which was measured in the culture supernatants, and this represents the secretion of cytokines from the cells (Sections 4.3.2-5). This section further looks at how the treatments affect intracellular cytokine production which was measured by intracellular binding of fluorescent anti-cytokine antibodies and detected on flow cytometry as cytokinepositive cells. Although methods to measure intracellular IL-12 failed to detect this cytokine (Appendix 6), the intracellular production of IL-6 and IL-10 was assessed in monocytes from healthy and MS groups. The effects of GA and IC treatments on intracellular IL-6 and IL-10 production were compared to that of in the LPS-stimulated monocytes, which served as a positive control (classical activation).

Classical activation of monocytes with LPS not only induced extracellular level of cytokines (Section 4.3.2), but also increased intracellular production of IL-6 and IL-10 in both the healthy and MS groups (Figure 4.6.a,b). When the levels were compared between the two groups, monocytes from MS patients had a lower level of IL-6 production after the LPS treatments, whereas, GA and IC showed no difference between the treatments, and higher level of IL-10 after stimulation with LPS only.

When monocytes were treated with GA, intracellular production of IL-10 was similar to that of classically activated monocytes in both healthy and MS groups, shown by a similar number of IL-10 positive cells (Figure 4.6.b) and a similar level of IL-10 per monocyte (Figure 4.6.c). This indicates that the increased level of extracellular IL-10 (Section 4.3.3) is well supported by a new intracellular production of this cytokine.

As shown previously, IC increased a production of extracellular IL-10 in both healthy and MS groups (section 4.3.4). When intracellular levels of cytokines were measured, intracellular IL-10 level in healthy IC-treated monocytes was similar to that of classically activated monocytes; however, in MS patients' monocytes the production was lower (Figure 4.6.b). When the differences between healthy and MS groups were assessed, the intracellular production of IL-10 was similar in both groups, indicating that the production of IL-10 in MS patients' IC-treated monocytes is at normal level. In addition, an intracellular production of IL-10 per each monocyte remained high in both healthy and MS groups (Figure 4.6.c), indicating that IC treatment of monocytes from MS group maintains a high intracellular 
production of IL-10, shown by both high percentage of IL-10 producing monocytes and the production of IL-10 in each monocyte.

A combination treatment of GA and IC in monocytes induced high intracellular production of IL-10 in healthy subjects compared to the single treatments; however, in MS patients, the production remained similar to GA or IC single treatments and was lower than in that of the healthy group. This indicates that, although a combination treatment with GA and IC further increases a high production of intracellular IL-10, observed in the single GA and IC treatments of monocytes from healthy subjects, there is no such improvement in monocytes from MS patients. In summary, despite monocytes from MS patients having produced an overall lower level of intracellular IL-6 after all of the treatments, the levels were not altered by GA and IC. In contrast, the intracellular IL-10 cytokine was constitutively produced by monocytes and no treatment induced a significant change in this cytokine. While GA and IC together modestly enhanced IL-10 in monocytes from healthy subjects, LPS alone had the greatest effect effect on IL-10 in MS patients. 
a.

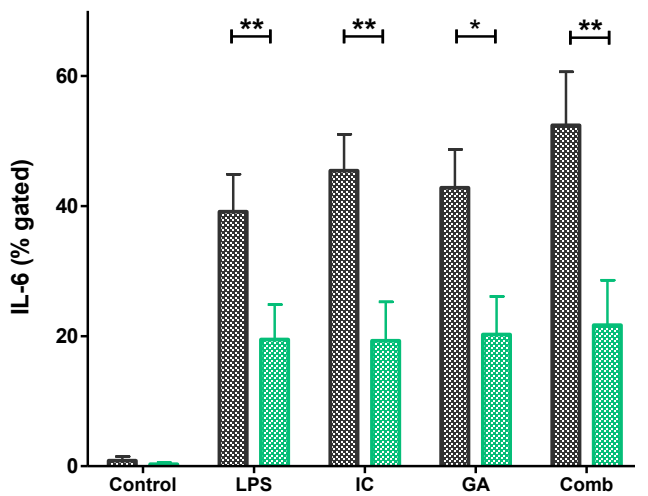

b.

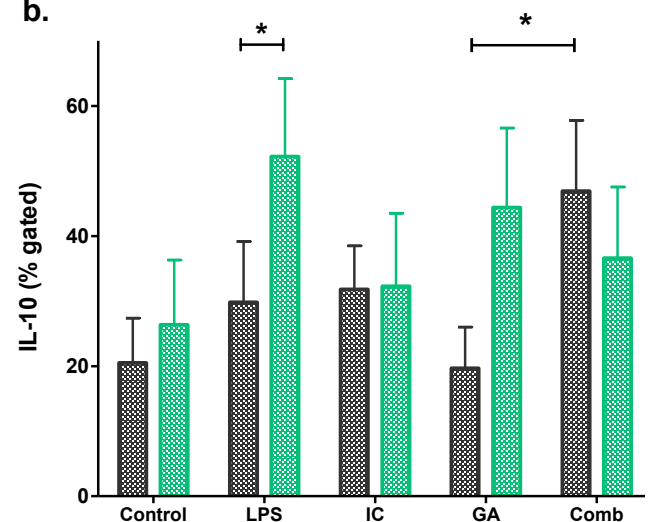

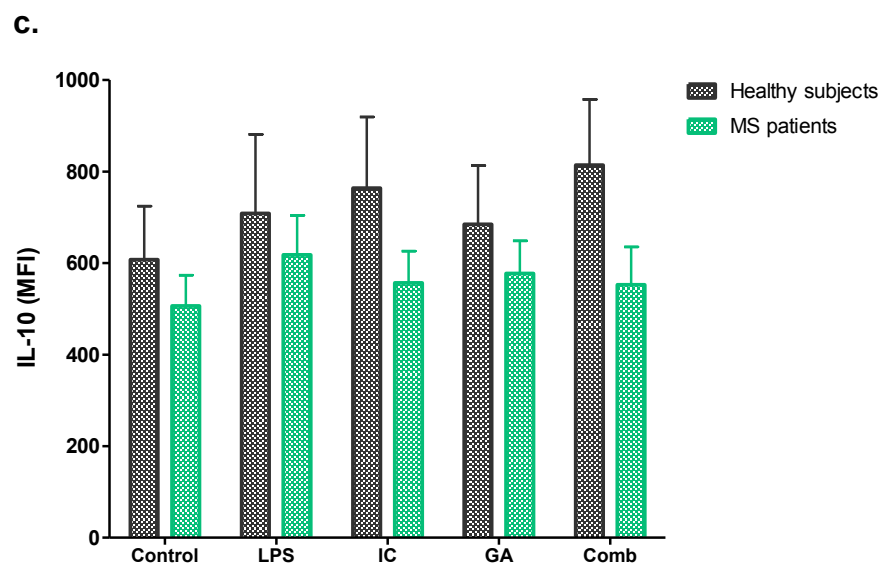

Figure 4.6. Intracellular cytokine production in monocytes after GA and IC treatments.

Total monocytes from healthy subjects $(n=8)$ and MS patients $(n=7)$ were primed with $20 \mathrm{U} / \mathrm{ml}$ IFN- $\gamma$ overnight and incubated with LPS, GA or IC in the presence of monensin at a final concentration of $0.67 \mu 1 / \mathrm{ml} / 10^{6}$ cells for 22 hours. The monocytes were then harvested and stained with fluorescent anti-CD14 and anti-CD16 antibodies. The cells were fixed in $100 \mu \mathrm{l}$ of $4 \%$ paraformaldehyde with subsequent permeabilization in $0.1 \%$ saponin. A 30 min intracellular staining was performed using fluorescent anti-cytokine antibodies and the data was acquired by flow cytometry and analyzed using FlowJo 5 software. The control group represents unstimulated and untreated monocytes. (a) Production of intracellular IL-6 in monocytes shown as a percentage of positive cells (\% gated). (b) Production of intracellular IL-10 in monocytes shown as a percentage of positive cells (\% gated). (c) Production of intracellular IL-10 per monocyte shown as mean fluorescent intensity of positive cells (MFI). Shown are the means and SD of individual experiments in healthy and MS groups. The statistical analysing was done by using one way ANOVA with Dunnett's post-test to compare three or more groups and Student's t-test to compare 2 groups. ${ }^{*} \mathrm{P}<0.01 ; * * \mathrm{P}<0.005$. Data were collected on flow cytometry using the gating strategy in appendix 9. 


\subsubsection{Validation of results by PCR}

To further validate the changes in cytokine and activation marker expression at the gene level, mRNA expression was examined by qPCR. Although GA-treated monocytes from both healthy and MS groups did not have altered mRNA expression of HLA-DR, IL-6 and IL-10 (Figures 4.7.c,e,f), the transcript levels of CD40, CD86 and IL-12 were downregulated after the treatment (Figures 4.7.a,b,d). These results confirm the previous findings that assessed cytokine production by ELISA and surface marker expression by flow cytometry.

IC treatment of monocytes in both healthy and MS groups inhibited expression of CD40, CD86 and HLA-DR mRNA (Figures 4.7.a-c) and increased the expression of IL-10 (Figure 4.7.f) as previously observed. Although IL-12 levels measured by ELISA were not altered by IC treatment, the mRNA expression of IL-12 was significantly downregulated by the treatment (Figure 4.7.d) in both groups. This finding indicates that the expression of this cytokine is regulated by IC, at least at the gene level. IC and GA combination treatment of monocytes from both groups resulted in reduced mRNA expression of CD40, CD86, HLADR and IL-12 (Figures 4.7.a-d), compared to classically activated monocytes, but did not change the expressions of IL-6 and IL-10 (Figures 4.7.e,f). Taken together these results are in agreement with the type II activating effect of IC, GA, or combination treatment as observed previously in protein levels (Section 4.3.3-5). 


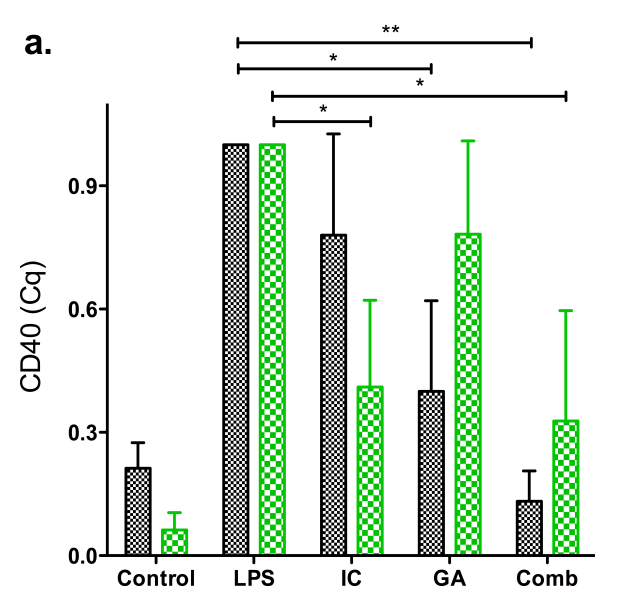

c.

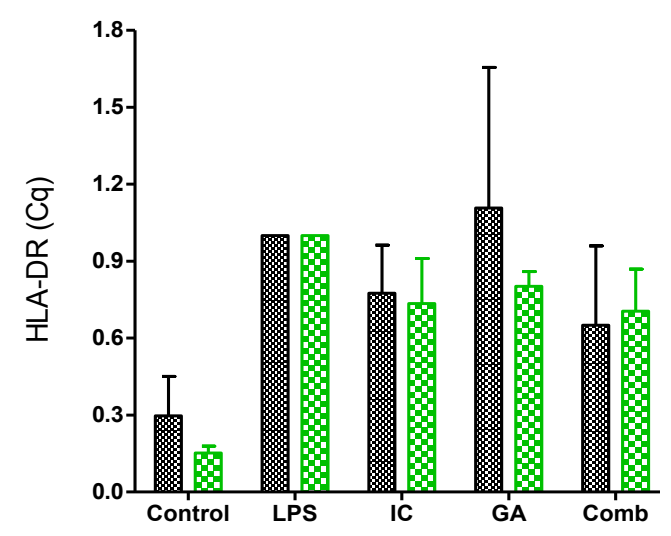

e.

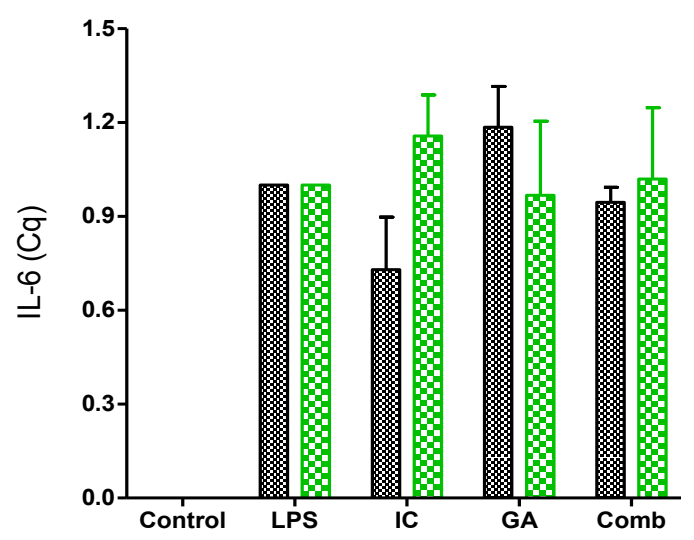

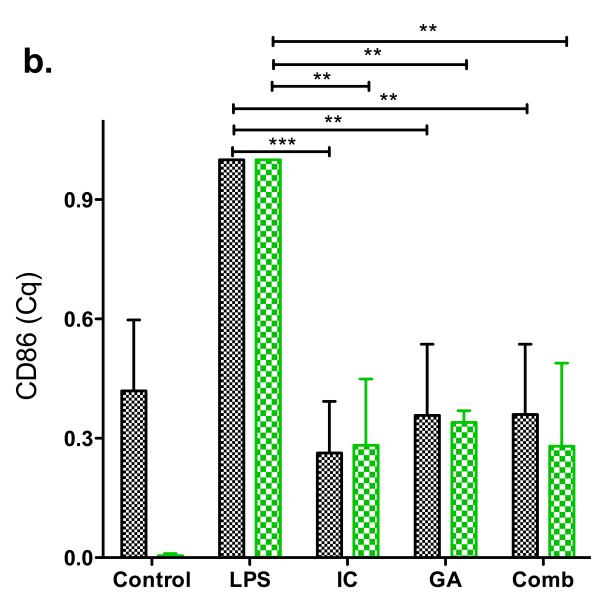

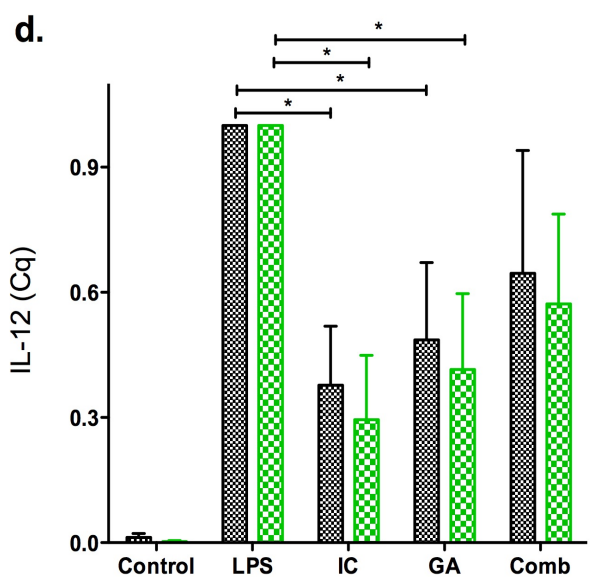

f.

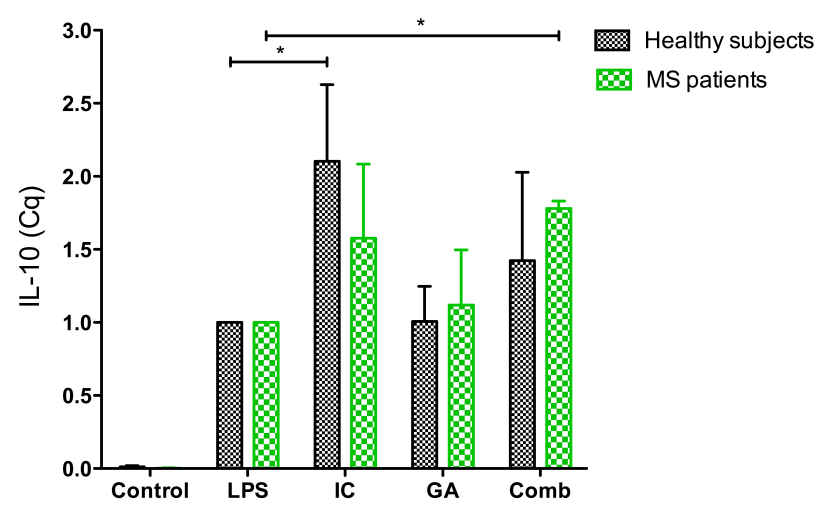

Figure 4.7. qRT-PCR validation of results: mRNA expression of markers and cytokines in monocytes.

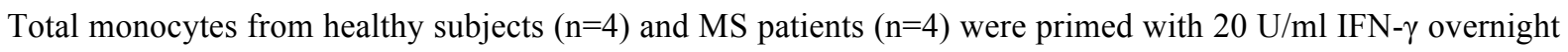
and treated with GA and/or IC in the presence or absence of $200 \mathrm{ng} / \mathrm{ml}$ LPS for 24 hours. The monocytes were then harvested and assessed for mRNA expressions by qRT-PCR (for methods see chapter 2.11.2). The mRNA expression is shown as a relative $\mathrm{Cq}$ expression $\left(2^{-\Delta \Delta \mathrm{Cq}}\right)$. The control group represents unstimulated and untreated monocytes. (a) CD40 mRNA expression in monocytes. (b) CD86 mRNA expression in monocytes. (c) HLA-DR mRNA expression in monocytes. (d) IL-12 mRNA expression in monocytes. (e) IL-6 mRNA expression in monocytes. (f) IL-10 mRNA expression in monocytes. Shown are the means and SD of duplicate wells from individuals in healthy and MS groups. The statistical analysing was done by using one way ANOVA to compare three or more groups and Student's t-test to compare 2 groups. 


\subsection{DISCUSSIONS}

This study investigated the activation of monocytes by in vitro treatment with LPS, GA and IC. Additonally, the phenotype of monocytes, type II activated by GA and IC, was characterized in both healthy subjects and MS patients. .

\subsubsection{In vitro classical activation of monocytes in MS}

Monocytes from healthy subjects can be classically activated upon LPS stimulation in vitro as shown by the induction of inflammatory cytokines and molecules[199]. In agreement with this study, LPS-treated healthy monocytes in our study showed typical characteristics of classically activated monocytes, such as active production of cytokines and inflammatory markers. Interestingly, inflammatory monocytes from MS patients showed an enhanced ability to become classically activated, in comparison to healthy monocytes. Moreover, classically activated MS patients' monocytes had a higher increase in the production of inflammatory cytokines and markers such as IL-6, CD40, CD86 and HLA-DR than that of healthy subjects. This suggests that monocytes from MS patients have a higher capacity to further induce inflammation, compared to healthy monocytes, possibly because they have already been previously activated in vivo during the course of MS (Chapter 3). These results not only confirm the previous observation, in which MS patients' monocytes were more inflammatory than the healthy monocytes (Chapter 3), but also demonstrate that their inflammatory ability can be further increased, if they encounter an inflammatory stimulant such as LPS in vitro.

\subsubsection{In vitro GA effect on monocytes in MS}

GA is currently used in the treatment of MS, primarily for RRMS; however, it only has about $30 \%$ efficacy[156]. In order to improve its efficacy it is essential to fully understand its mechanism of action. While Th1 and Th17 cells, and monocytes play the key role in CNS inflammation in MS[24, 31, 32], GA can directly affect both T cells and monocytes. The effect of GA on T cells is relatively well understood[157, 158, 162, 163, 166], however, none of these studies have explained the full spectrum of GA effects on the immune system, and 
exploration of GA effect on monocytes would give a broader picture of its mechanism of action[21, 32].

Recently, a regulatory effect of GA on monocytes has been determined. In vitro treatment of monocytes with GA results in inhibited production of inflammatory cytokines and molecules, such as TNF $\alpha$ and SLAM[32]. Furthermore, monocytes exposed to GA in the peripheral blood, directly inhibit Th1 cell proliferation in mouse model of MS[166]. In conjunction, our study gives additional insight into the direct effect of GA on human MS monocytes in vitro. As such, GA significantly downregulated important inflammatory products in monocytes, including CD40, CD86 and IL-12 at both the mRNA and protein levels, indicating type II activation of monocytes by GA. According to Kim et al., type II activated monocytes inhibit Th1 cell activation and polarize the $\mathrm{T}$ cell profile towards the Th2 side[21]. Our study confirms these observations and suggests that GA specifically targets the co-stimulatory and subset differentiation pathways for $\mathrm{T}$ cell activation in monocytes. The benefits of type II activated monocytes are not restricted to the polarization of T cells. It is suggested that for GA to exhibit its effect in MS, T cell polarization is not necessarily required[32]. Malefyt et al. have shown that regulatory monocytes can inhibit classically activated monocytes by producing anti-inflammatory cytokines[200].

As the type II activation by GA was induced in the presence of LPS in this study, it is likely that GA-treated monocytes can modulate the inflammatory monocytes in MS. Whether the GA-activated monocytes can regulate inflammatory monocytes in the periphery or in the site of inflammation, is yet to be determined. Toker et al. indicate that the alternative routes of GA administration might alter GA efficacy[166]. Clinically GA is administered via subcutaneous injection. As modulation of monocytes by GA is an important mechanism of its action, an intravenous injection of GA can be considered as an alternative approach for GA treatment, as it would allow GA to encounter the highest number of monocytes in the circulation. In conclusion, this study shows that GA has a direct type II activating effect on human monocytes in MS and mainly affects their co-stimulatory marker expressions and inflammatory cytokine production.

\subsubsection{In vitro IC effect on monocytes in MS}

IC have a direct type II activating effect on mouse tissue resident monocytes (macrophages) and improve the disease course in a mouse model of MS[44]. Moreover, 
ligation of Fc $\gamma$ receptors with IC results in inhibition of inflammatory IL-12 cytokine production in mice macrophages[197, 201], indicating a direct anti-inflammatory effect of IC on the differentiated monocytes (macrophages). Within humans, IC may be formed upon intravenous IVIG injection[174-176], and monocytes can effectively bind to them[178], and thus, the in vitro IC treatment of monocytes may replicate the in vivo activation of monocytes by IVIG. IVIG has been shown to reduce relapse rates and clinical symptoms in MS patients [146-149]. However, there are no sufficient reports about the mechanism of action of IVIG and IC in MS.

This study shows that IC has a type II activating effect on human monocytes in MS. The effect of IC on monocytes from MS patients was slightly different from that of healthy subjects. Whereas monocytes from healthy subjects had downregulated levels of CD40 and HLA-DR after IC treatment, IC inhibited the expression of CD40 and CD86, but not HLADR in MS patients. This decrease in CD40 and CD86 levels was also observed at the gene level. Gille et al. have shown that neonatal cord blood monocytes have significantly downregulated expression of CD86 and HLA-DR, whereas in peripheral blood monocytes the inhibition is not significant after IVIG treatment[202]. Our study supports the observation of downregulation of CD40 on neonatal cord blood monocytes; while we found no significant inhibitory effect of IC on CD86 expression similar to the work in peripheral blood human monocytes. Thus, the location of monocytes within humans may affect the IC effect on CD86 and HLA-DR expression. Despite this, our study has shown that the inhibitory effect of IC on CD40 is significant and supports the previous mouse study which has shown that IC inhibits the expression of CD40 in mice macrophages in vitro[44]. CD40 is a crucial mediator of T cell activation and potent enough to define the polarization of T cells[203], high expression of which activates Th1 cells; whereas, a low level of CD40 activates Th2 cells[44]. As the activation of Th2 cells is inhibitory to Th1 cells and beneficial in MS[21], the downregulated expression of CD40 can be considered one of the key anti-inflammatory mechanisms of IC treatment in MS. To date there have been no studies showing the IC effect on surface marker expression in monocytes, and our study has shown that IC can type II activate monocytes by mainly targeting co-stimulatory pathways. Both CD40 and CD86 are important markers, responsible for the co-stimulation of $\mathrm{T}$ cells and their binding to $\mathrm{T}$ cells is a key factor for their stimulation $[52,71]$. Therefore, the mechanism for the improvement of MS, shown in the clinical trials[146-149], could be due to the suppression of inflammatory $\mathrm{T}$ cell activation through the inhibition of co-stimulatory molecules CD40 and CD86. 
Previously it was reported that IC inhibits IL-12 production in mice macrophages[197, 201]. In this study however, the levels of inflammatory IL-12 cytokine, as well as IL-6, were not altered by IC. This difference may be due to the different level of monocyte differentiation, as the previous studies have looked at macrophages, which are a highly mature form of monocytes. Alternatively, a high affinity receptor for IVIG (FcyRI or CD64) is highly expressed on mice monocytes, but lower on healthy human monocytes[41], and our study revealed that CD64 expression on MS monocytes is significantly higher than on healthy monocytes after treatment with IC (data not shown). Therefore, it is unlikely that the differential expression of CD64 in mice and human monocytes will affect IC efficacy. Moreover, CD64 is not the only receptor for IC. Among many FcyRs expressed on monocytes, FcrRIIb (CD32) is known to be the only inhibitory receptor[204] and has been shown to be an important mediator of the IVIG effect in autoimmune diseases[178, 205]. Therefore, the binding and efficacy of IC on inflammatory cytokine productions cannot be solely judged by the expression of a single type of Fc $\gamma$ Rs, but should rather be referred to as a balance between the activating and inhibitory Fc $\gamma$ Rs in human monocytes. Another possible explanation is that the time point in which the IVIG was administered to the monocyte cultures as this can affect its efficacy. Thus, the administration of IVIG at the start of the culturing results in the strongest inhibition of TNF $\alpha$ production in monocytes[206]. In this study however, a 16 hour pre-culture treatment with IFN- $\gamma$ was required in order to induce marker and cytokine production. Therefore, late administration of IC into monocyte cultures may be a reason for the lack of effect of IC on inflammatory cytokine production measured after 24 hours of IC treatment. However, the mRNA level of IL-12 was downregulated by IC, suggesting that longer or repetitive treatments may be a requirement for the IC to affect the IL-12 production at the protein level.

The production of anti-inflammatory cytokine IL-10 was increased in healthy subjects in response to IC, with a smaller increase in MS patients, and this was confirmed at both the protein and mRNA level. Although IC treatment upregulated the extracellular secretion of IL10 in monocytes from MS patients, the number of new IL-10 producing cells was lower than in the LPS-treated cells. As LPS treatment in this study served as a positive control for the IC treatment, the decrease in intracellular IL-10 was observed in the relation to the positive control only and not when compared to that of the healthy group. Therefore, although IC did not upregulate the number of IL-10 positive cells in relation to the positive control, the overall new production and secretion of IL-10 in monocytes from MS patients was at a similar high level as in the healthy subjects. This finding suggests that type II activated 
monocytes maintain a high secretion of IL-10 by a new intracellular production after IC treatment. In addition, the MS patients' monocytes that had downregulated levels of intracellular IL-10 had increased extracellular secretion of IL-10 in parallel. Therefore, it is possible, that in these patients, a long term treatment with IC is required to produce new intracellular IL-10.

In this study, MS patients' monocytes had higher levels of IL-10 both intracellularly and extracellularly than in the healthy group when the production of IL-10 was measured 24 hours post-stimulation with LPS. However, Malefyt et al. have observed the maximal IL-10 production after 24-48 hours of stimulation with LPS in monocytes from healthy subjects[200]. The early IL-10 production by the patients' monocytes in this study can be explained by the fact that MS patients' monocytes had been circulating in the state of activation during the course of disease [207], and their early activation might lead to higher IL-10 production upon additional 24 hour stimulation with LPS and IC. Alternatively, Filion et al. have found that IL-10 production is higher in monocytes of patients with secondary progressive MS, in comparison to patients with relapsing remitting MS or healthy controls[184]. In agreement with Filion et al., this study has shown that monocytes producing high levels of IL-10 were mainly from the patients with SPMS and profound disability. Although the mechanism for this high IL-10 level in progressive MS is unknown, it may be part of an adaptation mechanism by the monocytes. Overall, this study shows for the first time a direct anti-inflammatory and type II activating effect of IC on human monocytes in MS.

\subsubsection{In vitro IC and GA effect on monocytes in MS}

Multitherapy has been long considered as an attractive approach for the treatment of MS[208]. As IC and GA single treatments have various type II activating effects, this study further investigated the in vitro effect of IC and GA combination treatment on monocytes in MS. Combination treatment significantly downregulated expression of CD40, CD86 and the production of IL-12 in monocytes of MS patients at both the protein and mRNA levels. Additionally, although IL-10 production was not altered by the combination treatment, the mRNA expression of IL-10 was increased. This is different from the observations in the healthy group, in which IL-10 production was significantly increased after 24 hours treatment, although the increase in IL-10 mRNA was not as high as in MS patients. This 
suggests that MS patients' monocytes require longer treatment than is required for the monocytes from healthy subjects, in order to allow the increase in mRNA expression of IL10 to be reflected in the production of cytokines. This is shown by the fact that the IL-10 mRNA increase is higher in monocytes after the combination treatment, than after single IC or GA treatments in MS patients. This finding indicates that the combination treatment improves the effects of IC and GA on MS patients' monocytes. Another factor to consider for IC and GA administration during the combination treatment is time. Although in this study the IC and GA have been administered simultaneously, it is possible that in the clinic a subsequent administration of the agents at different times may improve the type II activating effect of the combination treatment as monocytes from MS patients might respond to the IC earlier than the healthy monocytes. Overall, this study shows that GA and IC combination treatment has a direct type II activating effect on monocytes in MS.

\subsection{CONCLUSIONS}

a. This in vitro study shows that IC and GA have a direct type II activating effect on human monocytes in both healthy and MS groups.

b. A combination of IC and GA treatments had a better effect in the type II activation of monocytes, than the single IC or GA treatment. 
Chapter 5.

Type II activation of $\mathrm{CD}^{+}{ }^{+}$and $\mathrm{CD}^{+}{ }^{+}$monocyte subsets 


\subsection{INTRODUCTION}

Human $\mathrm{CD}_{1} 4^{+}$and $\mathrm{CD}_{16}{ }^{+}$monocyte subsets have significant phenotypical and functional differences. For example, healthy $\mathrm{CD} 14^{+}$monocytes express high levels of CCR2 and CD64[41, 79, 80], and produce high level of anti-inflammatory cytokine IL-10[81]. In contrast, healthy $\mathrm{CD} 16^{+}$monocytes have high expression of various inflammatory markers and cytokines[79, 80, 82-84], suggesting $\mathrm{CD} 16^{+}$monocytes have a highly inflammatory nature. During CNS inflammation, $\mathrm{CD} 16^{+}$monocytes and their differentiated forms (macrophages) have been shown to be more active in inducing inflammation than the CD14 monocytes[101-104].

The proportion of $\mathrm{CD} 14^{+}$and $\mathrm{CD} 16^{+}$monocytes changes during inflammation with an increase in the number of $\mathrm{CD} 16^{+}$but not $\mathrm{CD} 14^{+}$monocytes, and this increase in the $\mathrm{CD} 16^{+}$ subset correlates with the expansion of inflammatory Th1 cells and activation of inflammation[86, 88, 90, 94, 105, 209]. Despite the fact that the proportion of monocyte subsets significantly changes during MS with a 3 to 6 fold increase in the number of CD16 monocytes[97], the role of $\mathrm{CD} 16^{+}$monocytes in MS has not been elucidated yet.

Classically activated $\mathrm{CD} 14^{+}$and $\mathrm{CD} 16^{+}$monocytes play a role in inflammation and, in particular, it has been found that monocytes circulate in a state that resembles classical activation during MS[54]. Unlike classically activated monocytes, type II activated monocytes play an anti-inflammatory role, and the induction of type II activated monocytes is beneficial in the treatment of multiple sclerosis[21]. Type II activation can be induced by exposure of monocytes and macrophages to IC[44] or GA[32] in an inflammatory environment (i.e. stimulation by LPS and IFN- $\gamma$ ); however, the type II activation of the different monocyte subsets has not been defined yet. This chapter will characterize type II activation of $\mathrm{CD} 14^{+}$and $\mathrm{CD} 16^{+}$monocyte subsets by GA and IC in healthy subjects and in MS patients. 


\subsection{AIMS}

This study aims to investigate, for the first time, how $\mathrm{CD} 14^{+}$and $\mathrm{CD} 16^{+}$monocytes can be type II activated by GA and IC, and to compare this activation state in healthy subjects and MS patients. These broad aims will be addressed by the following three specific aims:

1. To characterize the in vitro classical activation state of $\mathrm{CD}_{1} 4^{+}$and $\mathrm{CD}_{1} 6^{+}$monocyte subsets from healthy subjects and MS patients.

2. To characterize the in vitro type II activation state of $\mathrm{CD} 14^{+}$and $\mathrm{CD} 16^{+}$monocyte subsets from healthy subjects and MS patients.

3. To compare the type II activation of $\mathrm{CD} 14^{+}$and $\mathrm{CD}_{1} 6^{+}$monocyte subsets in purified cultures and co-cultures with either subset. 


\subsection{RESULTS}

\subsubsection{Classical activation of $\mathrm{CD}^{+} 4^{+}$and $\mathrm{CD}_{16}{ }^{+}$monocytes in vitro}

\subsubsection{Classical activation of $\mathrm{CD} 14^{+}$monocytes}

To induce classical activation, the purified monocyte subsets have been primed with IFN$\gamma$ overnight and treated with LPS for 24 hours. It has been previously shown that the classically (or type I) activated human monocytes obtain characteristics of inflammatory cells and express increased levels of CD40, CD86, HLA-DR, IL-12, IL-6 and IL-10 (Chapter 4). This study uses the same approach to characterize the classical activation of $\mathrm{CD}_{1} 4^{+}$and $\mathrm{CD}_{16}{ }^{+}$monocytes induced by the LPS stimulation.

In unstimulated $\mathrm{CD} 14^{+}$monocytes from healthy subjects, only basal levels of the activation markers are expressed and no production of cytokines is detected (Figure 5.1). In MS patients, however, unstimulated $\mathrm{CD} 14^{+}$monocytes have a significantly higher basal expression of CD40 and HLA-DR and low but detectable levels of the cytokines IL-12, IL-6 and IL-10 (Figure 5.1). When stimulated with LPS, healthy CD14 ${ }^{+}$monocytes express significantly higher levels of CD40, CD86, IL-12, IL-6 and IL-10 in comparison to the untreated cells (Figure 5.1). In LPS-treated CD14 ${ }^{+}$monocytes from MS patients HLA-DR, IL-6, IL-10 and IL-12 are upregulated, compared to untreated monocytes, and in particular, the levels of HLA-DR and IL-6 are higher than in those from the LPS-stimulated healthy subjects (Figure 5.1). Interestingly, the high basal expression of CD40 and CD86 could not be further enhanced by the classical activation of the monocytes from MS patients. Finally, the LPS stimulation does not change the expressions of CCR2 and CD64 on CD14 monocytes from either groups (Figure 5.1g and h). These findings indicate that while CD14 ${ }^{+}$ monocytes from MS patients express higher basal levels of CD40 and CD86, suggestive of higher basal activation state, $\mathrm{CD} 14^{+}$monocytes from both groups can be classically activated with MS CD $14^{+}$monocytes expressing higher levels of HLA-DR and IL-6. 

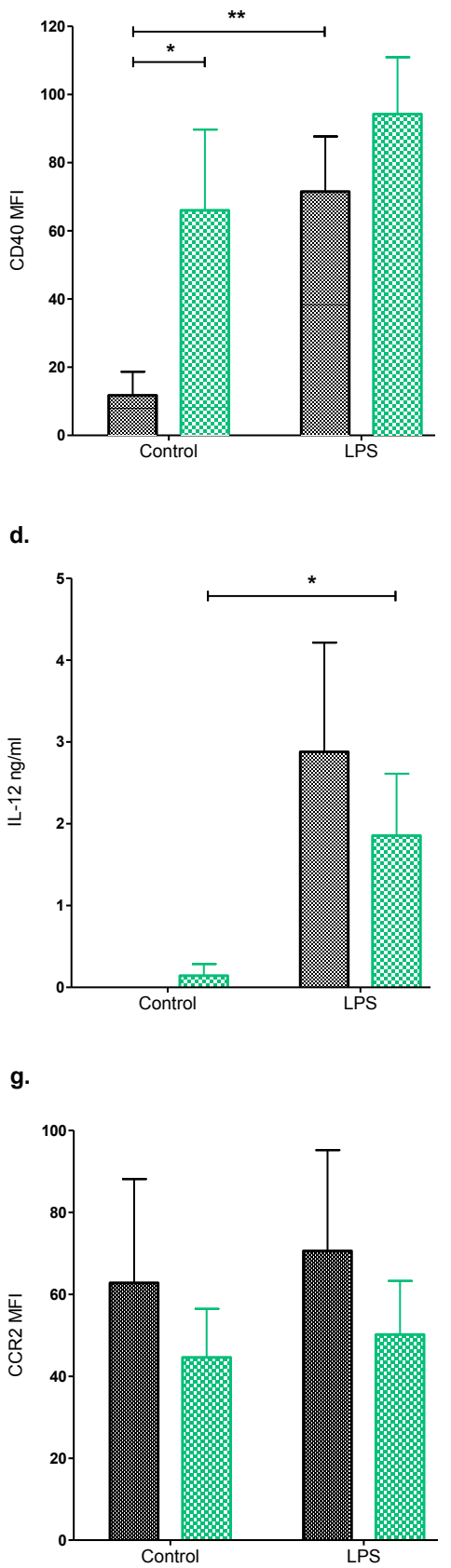

b.

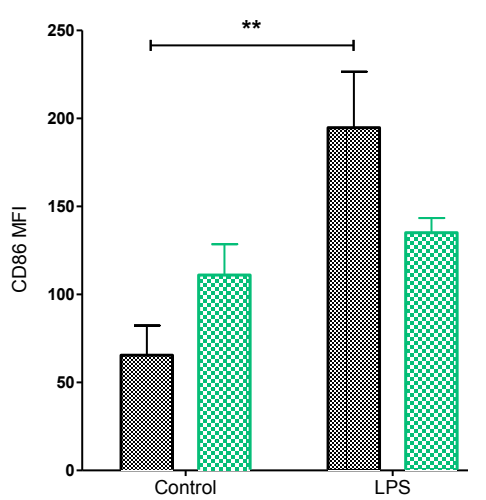

e.

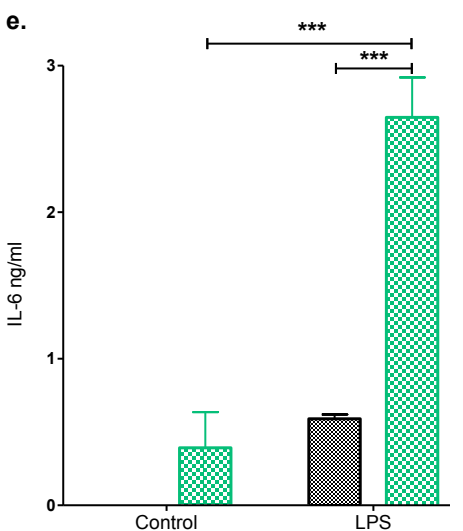

h.

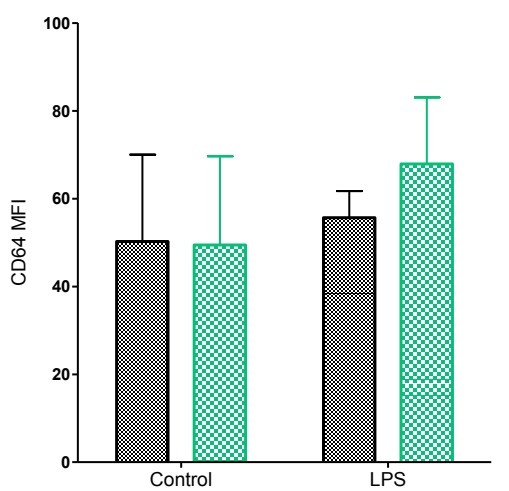

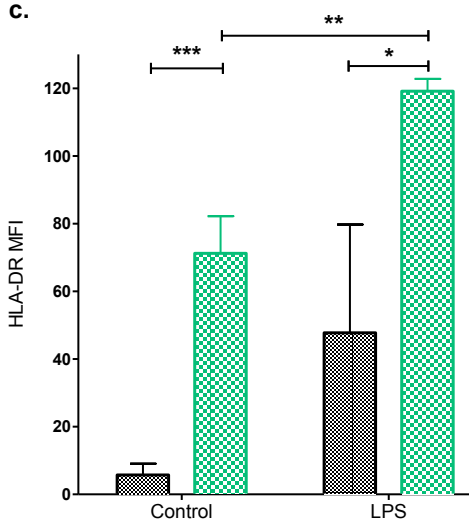

f.

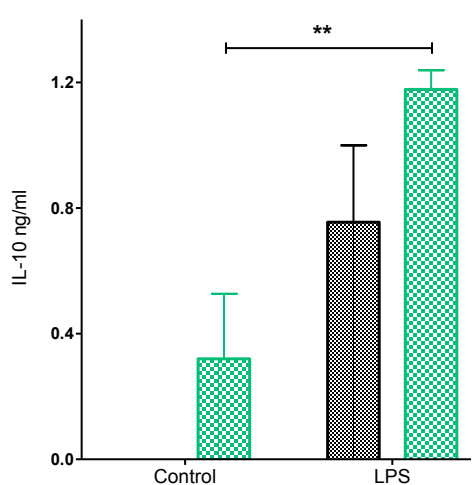

Healthy subjects

MS patients

Figure 5.1. CD14 ${ }^{+}$monocytes were classically activated by LPS in both healthy and MS groups.

$\mathrm{CD}_{14}{ }^{+}$monocytes from healthy subjects $(n=4)$ and MS patients $(n=4)$ were primed with $20 \mathrm{U} / \mathrm{ml}$ IFN- $\gamma$ overnight and stimulated with $200 \mathrm{ng} / \mathrm{ml}$ LPS for 24 hours. The monocytes were then harvested and stained with antibodies. The activation marker expression was assessed by flow cytometry, and cytokine production was measured by ELISA. The control group represents unstimulated and untreated monocytes. (a-h) The expressions of markers and cytokines in CD14 monocytes: (a) CD40, (b) CD86, (c) HLA-DR, (d) IL-12, (e) IL-6, (f) IL-10, (g) CCR2, and (h) CD64. Shown are the means and SD of duplicate wells from individuals in healthy and MS groups. ${ }^{*} \mathrm{p}<0.01 ; * * \mathrm{p}<0.005 ; * * * \mathrm{p}<0.001$ by one way ANOVA with Dunnett's post-test. Data were collected on flow cytometry using the gating strategy in appendix 10 . 


\subsubsection{Classical activation of $\mathrm{CD} 16^{+}$monocytes}

$\mathrm{CD}^{+} 6^{+}$monocytes from both healthy and MS patients expressed higher basal levels of CD40, CD86 and HLA-DR, than $\mathrm{CD}^{+} 4^{+}$monocytes, and produced low but detectable amounts of IL-6, IL-10 and IL-12 (Figures 5.2a-f). Classical activation induced the expression of CD40, IL-6, IL-10 and IL-12 in monocytes from both groups, while HLA-DR, CCR2 and CD64 were only upregulated on $\mathrm{CD}^{+} 6^{+}$monocytes from the MS group. Comparing the two groups, LPS-stimulated $\mathrm{CD} 16^{+}$monocytes from MS patients have levels of HLA-DR, IL-6 and IL-10 that are 2-, 5- and 4-fold higher than in healthy people (Figures 5.2.c, e, and f). Despite differences in the absolute levels and patterns of activation, CD16 ${ }^{+}$ monocytes from both healthy people and MS patients had characteristics of classically activated monocytes after priming with IFN- $\gamma$ and stimulation with LPS. In particular, these findings are based upon the significant induction of CD40 and the production of IL-6, IL-10 and IL-12 as measured in the culture supernatants. 


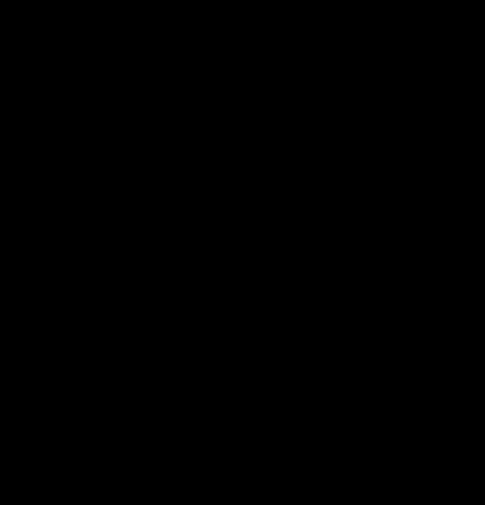

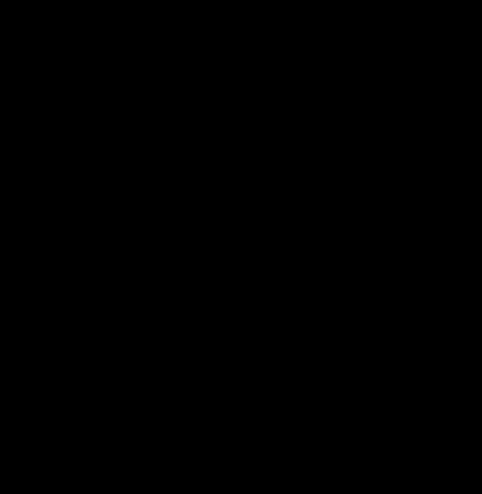

c.

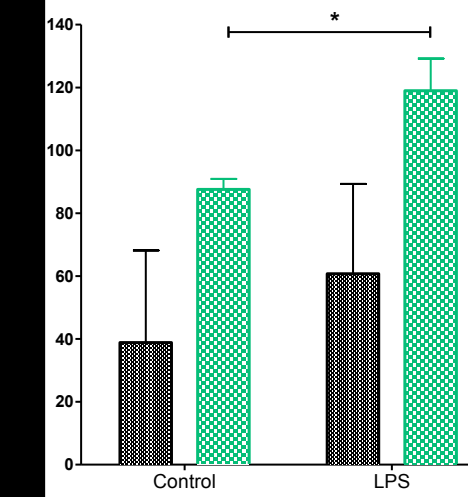

d.
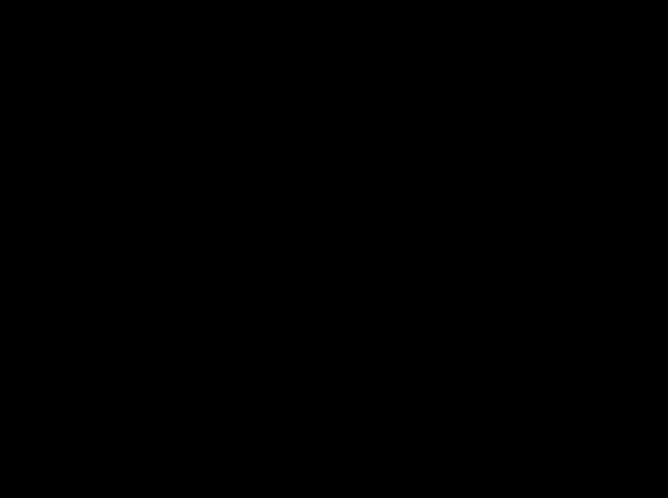

Figure 5.2. CD16 ${ }^{+}$monocytes were classically activated by LPS in both healthy and MS groups.

$\mathrm{CD}_{1} 6^{+}$monocytes from healthy subjects $(n=4)$ and MS patients $(n=4)$ were primed with $20 \mathrm{U} / \mathrm{ml}$ IFN- $\gamma$ overnight and stimulated with $200 \mathrm{ng} / \mathrm{ml}$ LPS for 24 hours. The monocytes were then harvested and stained with antibodies. The activation marker expression was assessed by flow cytometry and the cytokine production was measured by ELISA. The control group represents unstimulated and untreated monocytes. (a-h) The expressions of markers and cytokines in CD16 ${ }^{+}$monocytes: (a) CD40, (b) CD86, (c) HLA-DR, (d) IL-12, (e) IL-6, (f) IL-10, (g) CCR2, and (h) CD64. Shown are the means and SD of duplicate wells from individuals in healthy and MS groups. ${ }^{*} \mathrm{p}<0.01 ; * * \mathrm{p}<0.005 ; * * * \mathrm{p}<0.001$ by one way ANOVA with Dunnett's post-test. Data were collected on flow cytometry using the gating strategy in appendix 11. 


\subsubsection{Comparison between the classical activation of $\mathrm{CD} 14^{+}$and $\mathrm{CD} 16^{+}$monocytes}

When comparing the basal state of $\mathrm{CD} 14^{+}$and $\mathrm{CD}_{1} 6^{+}$monocytes from healthy subjects, $\mathrm{CD}_{16}{ }^{+}$monocytes had significantly higher levels of CD40, CD86 and IL-6, than CD14 monocytes, which expressed only low levels of these markers and cytokine (Figures 5.3.a,b, and e). None of the subsets produced any IL-10 and IL-12 basally and both expressed similar levels of CCR2 and CD64 even after LPS stimulation (Figures 5.3d and f-h). When stimulated with LPS, the two subsets expressed similar levels of activation markers and cytokines except IL-10 (Figure 5.3f). The classically activated CD14 $4^{+}$monocytes produced 6-fold higher amounts of IL-10 than the $\mathrm{CD} 16^{+}$monocytes despite producing similar levels of inflammatory cytokines IL-6 and IL-12 (Figures 5.3d and e). These results suggest that $\mathrm{CD}_{16}{ }^{+}$monocytes from healthy subjects have a more pro-inflammatory nature than $\mathrm{CD} 14^{+}$ monocytes even after classical activation. 
a.

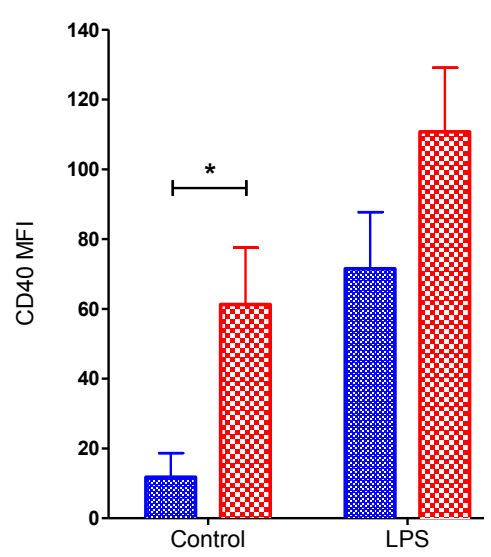

d.

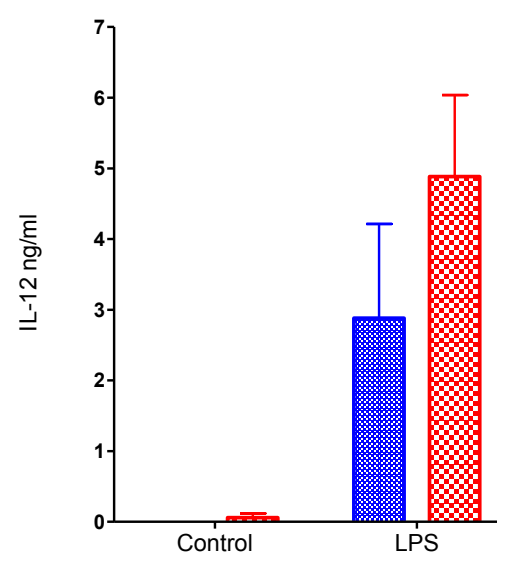

g.

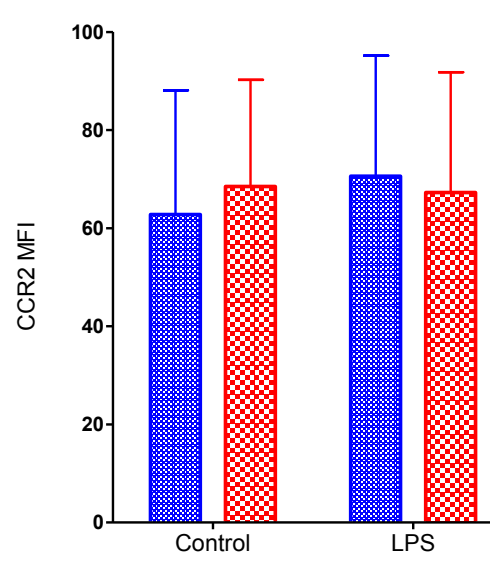

b.

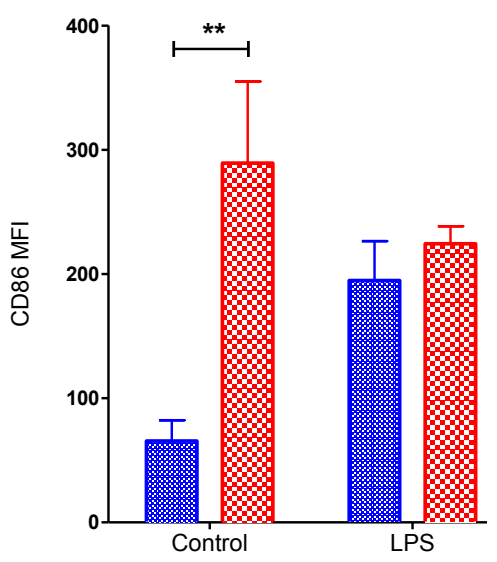

e.

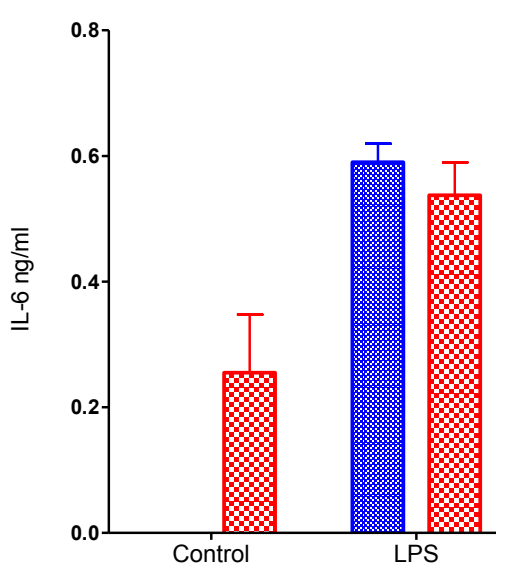

h.

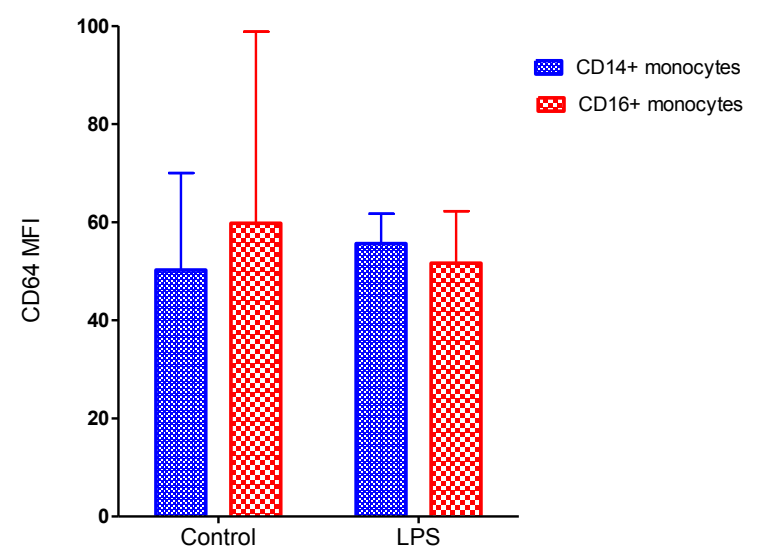

c.

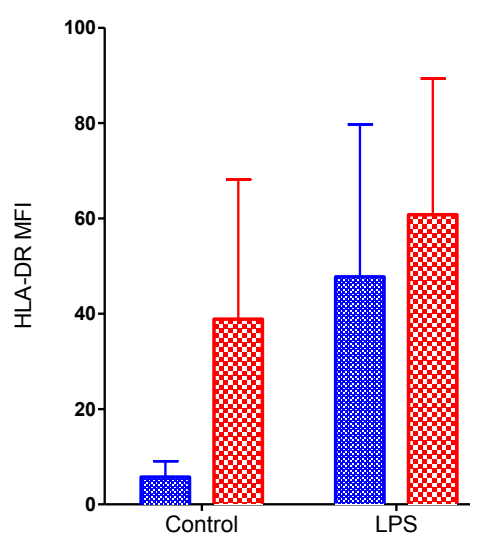

f.

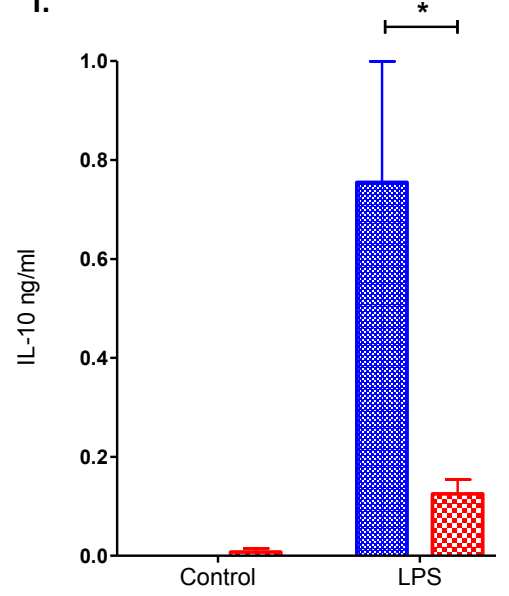

Figure 5.3. Healthy $\mathrm{CD} 14^{+}$and $\mathrm{CD}^{+} 6^{+}$monocytes were classically activated by LPS.

$\mathrm{CD}_{14}{ }^{+}$and $\mathrm{CD}_{1} 6^{+}$monocytes from healthy subjects $(\mathrm{n}=4)$ were primed with $20 \mathrm{U} / \mathrm{ml}$ IFN- $\gamma$ overnight and stimulated with $200 \mathrm{ng} / \mathrm{ml}$ LPS for 24 hours. The monocytes were then harvested and stained with antibodies. The activation marker expression was assessed by flow cytometry and the cytokine production was measured by ELISA. (a-h) The expressions of markers and cytokines in CD14 $4^{+}$and CD16 monocytes: (a) CD40, (b) CD86, (c) HLA-DR, (d) IL-12, (e) IL-6, (f) IL-10, (g) CCR2, and (h) CD64. Shown are the means and SD of duplicate wells from individuals in the healthy group. ${ }^{*} p<0.01$; * $p<0.005 ; * * * p<0.001$ by one way ANOVA with Dunnett's post-test. The graph is a representation of the same data as in the figures 5.1 and 5.2. Data were collected on flow cytometry using the gating strategies in appendices 10,11 . 
In contrast to monocytes from healthy subjects, only the basal expression of CD86 was significantly high in $\mathrm{CD}_{1} 6^{+}$monocytes from MS patients than the $\mathrm{CD} 14^{+}$subset (Figure 5.4b). However, as seen with monocytes from healthy subjects, CD14 ${ }^{+}$and CD16+ monocytes from MS patients expressed similar levels of activation markers and inflammatory cytokines except for IL-10 (Figure 5.4f). The IL-10 production in the CD16 $6^{+}$monocytes is significantly lower $(\sim 2$-fold $)$ than in the $\mathrm{CD} 14^{+}$monocytes. Taken together, these findings indicate that, while $\mathrm{CD} 14^{+}$monocytes from MS patients maintain the ability to produce higher levels of IL-10 upon LPS stimulation and have lower basal expression of CD86, the expression of other activation markers and cytokines is more similar to the $\mathrm{CD}_{16} 6^{+}$subset than when comparing monocytes from healthy subjects. 
a.

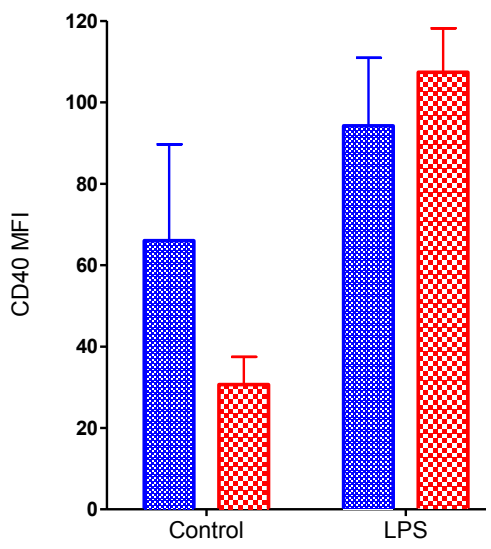

d.

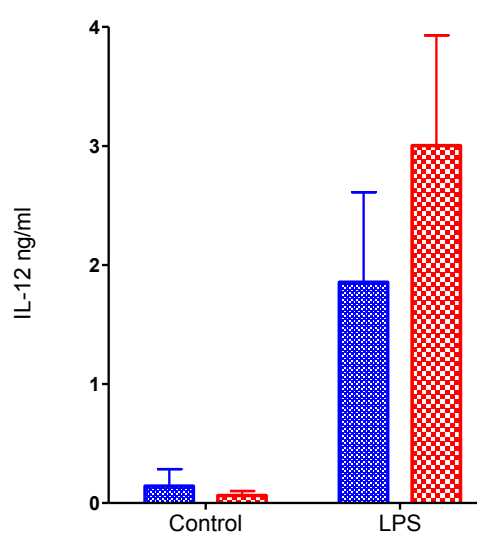

g.

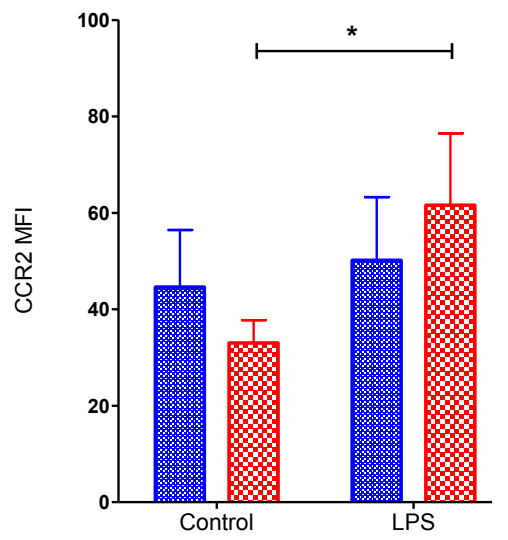

b.

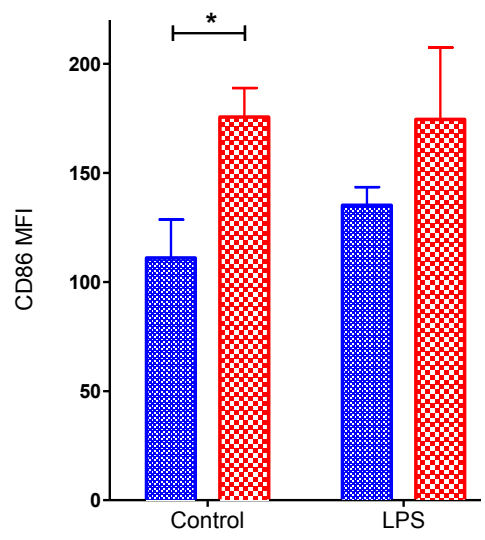

e.

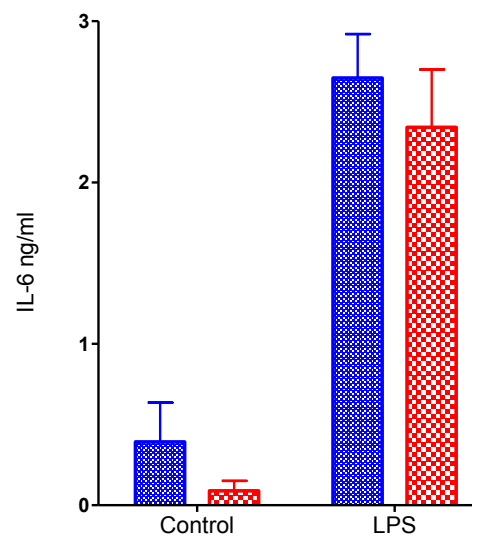

h.

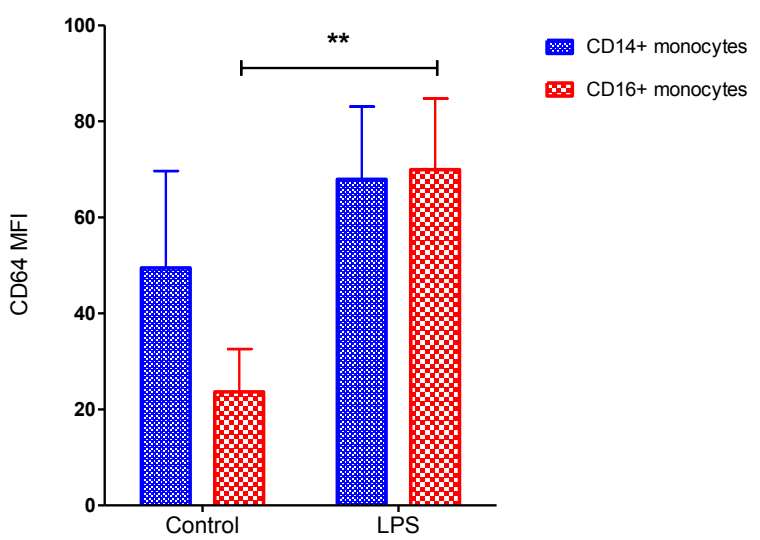

c.

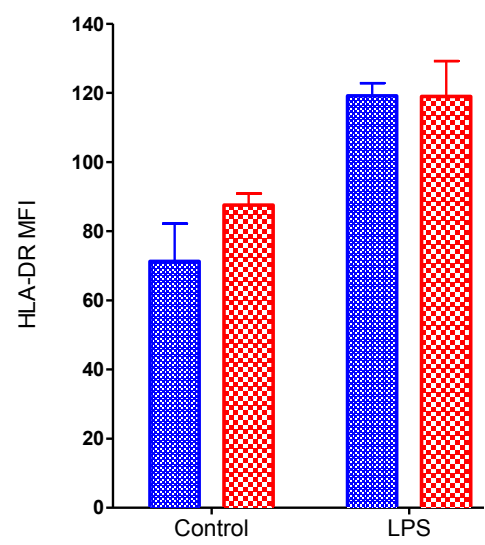

f.

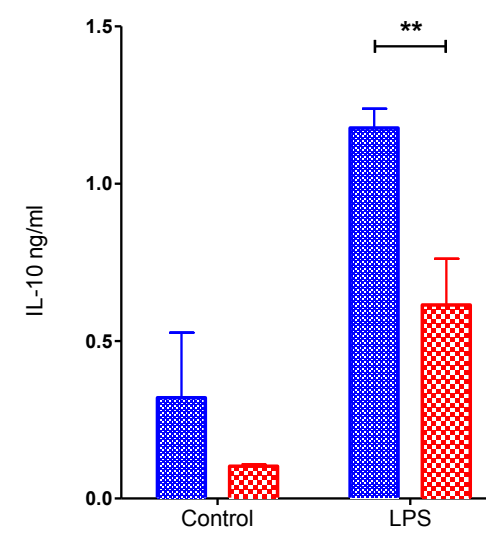

Figure 5.4. MS CD14 ${ }^{+}$and $\mathrm{CD}^{+}$monocytes were classically activated by LPS.

$\mathrm{CD}_{14}{ }^{+}$and $\mathrm{CD}_{1} 6^{+}$monocytes from MS patients $(\mathrm{n}=4)$ were primed with $20 \mathrm{U} / \mathrm{ml} \mathrm{IFN-} \gamma$ overnight and stimulated with $200 \mathrm{ng} / \mathrm{ml}$ LPS for 24 hours. The monocytes were then harvested and stained with antibodies. The activation marker expression was assessed by flow cytometry and the cytokine production was measured by ELISA. (a-h) The expressions of markers and cytokines in CD14 $4^{+}$and CD16 monocytes: (a) CD40, (b) CD86, (c) HLA-DR, (d) IL-12, (e) IL-6, (f) IL-10, (g) CCR2, and (h) CD64. Shown are the means and SD of duplicate wells from individuals in the MS group. ${ }^{*} \mathrm{p}<0.01 ; *{ }^{*}<<0.005 ; * * * p<0.001$ by using one way ANOVA with Dunnett's post-test. The graph is a representation of the same data as in the figures 5.1 and 5.2. Data were collected on flow cytometry using the gating strategies in appendices 10,11. 


\subsubsection{The effect of GA on $\mathrm{CD}^{+} 4^{+}$and $\mathrm{CD} 16^{+}$monocytes in vitro}

\subsubsection{The effect of GA on $\mathrm{CD} 14^{+}$and $\mathrm{CD} 16^{+}$monocytes}

The type II activation of $\mathrm{CD} 14^{+}$and $\mathrm{CD} 16^{+}$monocytes by GA was evaluated in purified monocyte subsets, primed with IFN- $\gamma$ and treated with GA in the presence of LPS, and this type II activation has been compared to classical activation (positive control). It had been shown previously in this study (Chapter 4) that the total monocyte populations from healthy and MS groups developed a type II activated phenotype after the treatment with GA. While the $\mathrm{CD} 14^{+}$monocytes from both healthy and MS groups had significantly decreased production of IL-12, only healthy $\mathrm{CD} 14^{+}$monocytes had a reduced expression of CD40. The expression of other cytokines and markers was not altered by the GA treatment of healthy (Figure 5.5) and MS CD $14^{+}$monocytes (Figure 5.6). Although $\mathrm{CD}_{16}{ }^{+}$monocytes from both healthy and MS groups had significantly decreased production of CD40 and IL-12, only $\mathrm{CD}_{16}{ }^{+}$monocytes from MS group had a reduced production of IL-6 (Figures 5.5 and 5.6).

\subsubsection{Comparison of the GA effect between $\mathrm{CD} 14^{+}$and $\mathrm{CD} 16^{+}$monocytes}

Healthy monocyte subsets maintained the differences in expression of CD40, HLA-DR, IL-10 and IL-12 after GA treatment between the CD14 ${ }^{+}$and $\mathrm{CD} 16^{+}$monocytes, in a similar level as in the classically activated subsets (Figure 5.5). However, the MS monocyte subsets maintained similar levels of CD40 and CD86 after GA treatment despite the difference observed between the classically activated $\mathrm{CD} 14^{+}$and $\mathrm{CD} 16^{+}$monocytes and retained the difference in the cytokine production (Figure 5.6). Thus, GA not only had a type II activating effect on monocyte subsets from both healthy and MS groups, it also balanced the difference in the activation marker expression between the $\mathrm{CD}_{1} 4^{+}$and $\mathrm{CD}_{1} 6^{+}$monocytes from $\mathrm{MS}$ patients. 


\subsubsection{The effect of $\mathrm{IC}$ on $\mathrm{CD}_{1}{ }^{+}$and $\mathrm{CD}^{+}{ }^{+}$monocytes in vitro}

\subsubsection{The effect of IC on $\mathrm{CD}_{14}^{+}$and $\mathrm{CD} 16^{+}$monocytes}

It has been previously shown that IC (immune complexes of intravenous immunoglobulin G and red blood cells) can type II activate human monocytes (Chapter 4). However, the type II activating effect of IC on monocyte subsets has not been studied. To address this question, the purified monocyte subsets were cultured individually, primed with IFN- $\gamma$ and treated with IC in the presence of LPS. The type II activation by IC was compared to classical activation, which also serves as a positive control. Although IC treatment of individually cultured $\mathrm{CD} 14^{+}$monocytes from healthy individuals had no demonstrable effect, the MS CD14 $4^{+}$monocytes expressed significantly lower CD40, CD86 and HLA-DR in the response to IC treatment. Additionally, cytokine production was not significantly altered by IC treatment of $\mathrm{CD} 14^{+}$monocytes (Figure 5.5). Although IC did not greatly affect healthy $\mathrm{CD} 16^{+}$monocytes, the MS CD16 ${ }^{+}$monocytes had not only a significant down-regulation of CD40, CD86 and HLA-DR expressions, but also a significant increase in IL-10 secretion in the response to IC (Figure 5.6).

\subsubsection{Comparison of the IC effect between $\mathrm{CD}^{+} 4^{+}$and $\mathrm{CD} 16^{+}$monocytes}

Classically activated $\mathrm{CD} 14^{+}$and $\mathrm{CD}_{1} 6^{+}$monocytes had been previously shown to have altered levels of cytokines and markers, and these differences were still prominent after the IC treatment of the subsets. As such, MS CD14 and $\mathrm{CD} 16^{+}$monocytes maintained the difference in the levels of CD40, CD86, IL-12, IL-6 and IL-10, while keeping the similarity in HLA-DR expression after the treatment with IC (Figure 5.6). Therefore, the CD14 ${ }^{+}$ monocytes remained the main producers of IL- 6 and IL-10, whereas the CD16 ${ }^{+}$monocytes sustained the high expression of CD40, CD86 and IL-12 after IC treatment, at the same level as classically activated $\mathrm{CD}_{1} 6^{+}$monocytes. Overall, the in vitro IC treatment of monocyte subsets from MS patients induces the type II activation of both monocyte subsets and yet maintains the phenotypical differences of the $\mathrm{CD} 14^{+}$and $\mathrm{CD} 16^{+}$monocytes. 


\subsubsection{The effect of combination treatment on subsets in vitro}

\subsubsection{The effect of GA and IC on CD14 ${ }^{+}$and CD16 ${ }^{+}$monocytes}

Although GA and IC have distinct type II activating effects on monocyte subsets, it was not clear whether GA and IC together could have a synergistic type II activating effect on $\mathrm{CD}_{14}{ }^{+}$and $\mathrm{CD} 16^{+}$monocytes. To address this question, the $\mathrm{CD} 14^{+}$and $\mathrm{CD} 16^{+}$monocytes were individually cultured, primed with IFN- $\gamma$ and treated with GA and IC in the presence of LPS. The production of markers and cytokines in the subsets, treated with GA and IC, was compared to that of classically activated monocytes. Whereas the monocyte subsets from healthy subjects did not show characteristics of type II activation, CD14 $4^{+}$and $\mathrm{CD} 16^{+}$ monocytes from MS patients had a type II activated phenotype in the response to combination treatment (Figure 5.6). Additionally, the $\mathrm{CD} 14^{+}$monocytes from MS patients had significantly decreased expression of CD40, CD86, HLA-DR and IL-12 after the combination treatment with GA and IC (Figure 5.6.a-d). Although the single GA and IC treatments did not show any effects on the IL-10 production, the combination treatment significantly upregulated IL-10 production in CD14 ${ }^{+}$monocytes from MS group. Similar to the $\mathrm{CD} 14^{+}$monocytes, $\mathrm{CD} 16^{+}$monocytes from MS patients had a reduced expression of CD40, CD86, HLA-DR, IL-12 and IL-6, and significantly upregulated production of IL-10 after the combination treatment (Figure 5.6).

\subsubsection{Comparison of the GA and IC effect between $\mathrm{CD} 14^{+}$and CD16 $6^{+}$monocytes}

Although the $\mathrm{CD} 14^{+}$and $\mathrm{CD} 16^{+}$monocytes from MS patients showed similar changes after the combination treatment, a difference was observed in IL-6 production between the subsets. Thus, the combination treatment inhibited IL-6 production in CD16 ${ }^{+}$monocytes from MS group but not in $\mathrm{CD} 14^{+}$monocytes. Additionally, the combination treatment was more effective at type II activating the $\mathrm{CD} 16^{+}$monocytes from MS patients, in which the decrease in the expressions of activation markers is 2-fold greater than in the CD14 ${ }^{+}$monocytes (Table 5.1). In conclusion, although the combination treatment did not have a significant effect on the monocyte subsets from healthy subjects, both $\mathrm{CD} 14^{+}$and $\mathrm{CD} 16^{+}$monocytes from MS patients were type II activated by the combined effects of GA and IC. 
a.

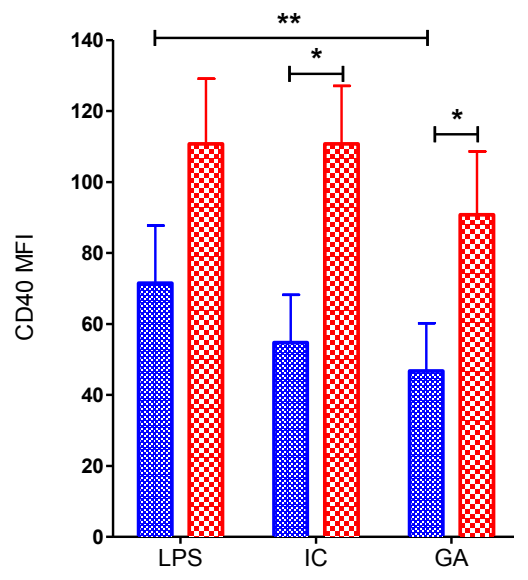

c.

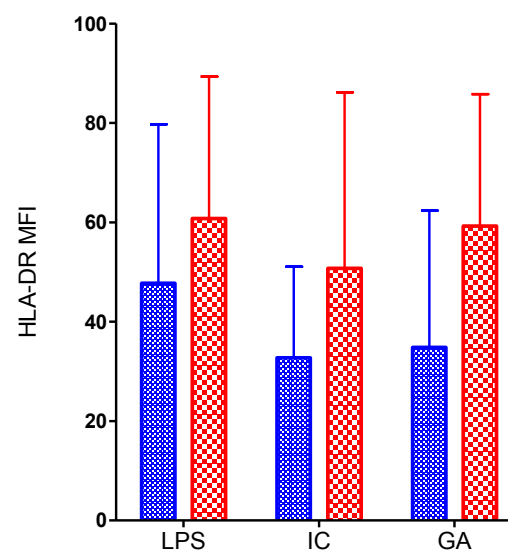

e.

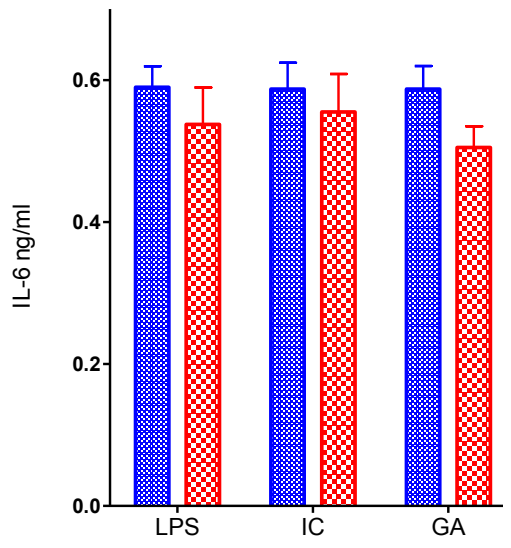

b.

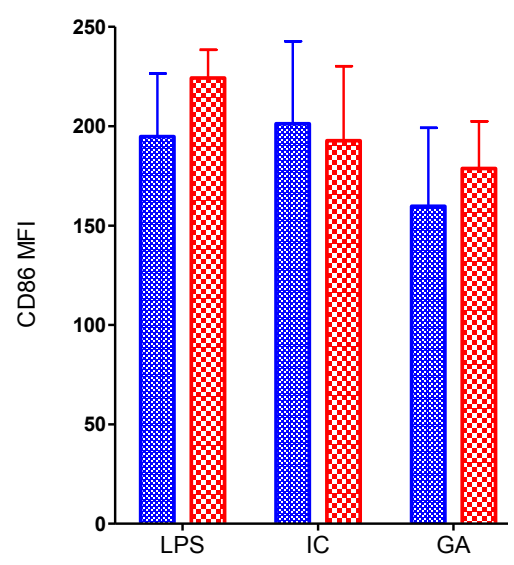

d.

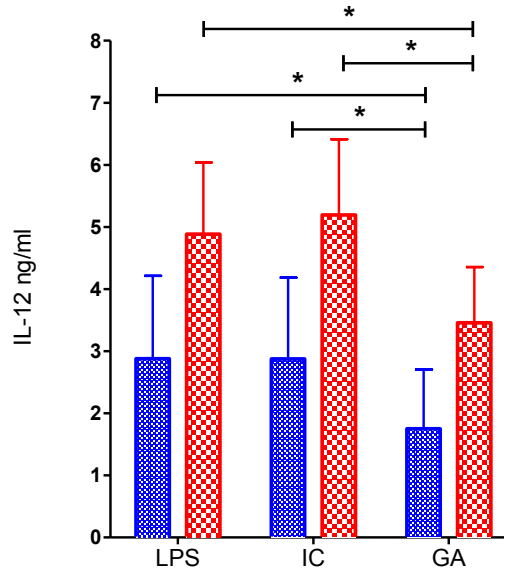

f.

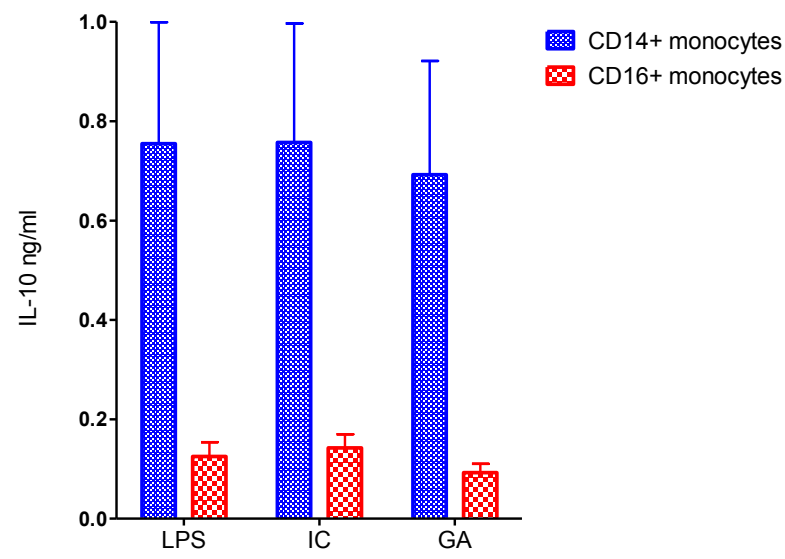

Figure 5.5. Type II activation of $\mathrm{CD}^{+} 4^{+}$and $\mathrm{CD}^{+}{ }^{+}$monocytes from healthy subjects.

$\mathrm{CD}_{14}{ }^{+}$and $\mathrm{CD}_{16} 6^{+}$monocytes from healthy subjects $(\mathrm{n}=4)$ were primed and treated with GA and/or IC in the presence of LPS for 24 hours. The monocytes were stained with antibodies, and the activation marker expression was assessed by flow cytometry. Cytokine production was measured by ELISA. (a-f) The expressions of markers and cytokines in subsets: (a) CD40, (b) CD86, (c) HLA-DR, (d) IL-12, (e) IL-6, and (f) IL-10. Shown are the means and SD of 4 individuals in the healthy group. *p $<0.01 ; * * p<0.005 ; * * * p<0.001$ by one way ANOVA with Dunnett's post-test. Data were collected on flow cytometry using the gating strategies in appendices 10,11 . 
a.

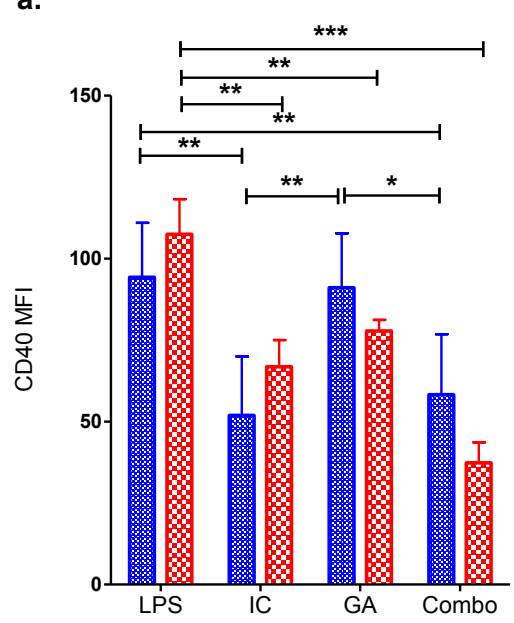

d.

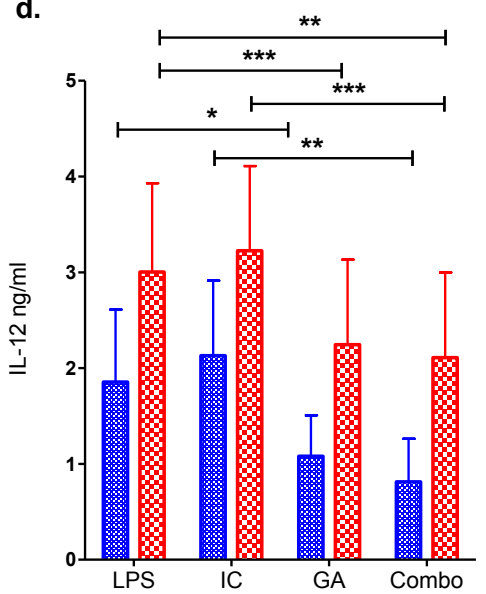

b.

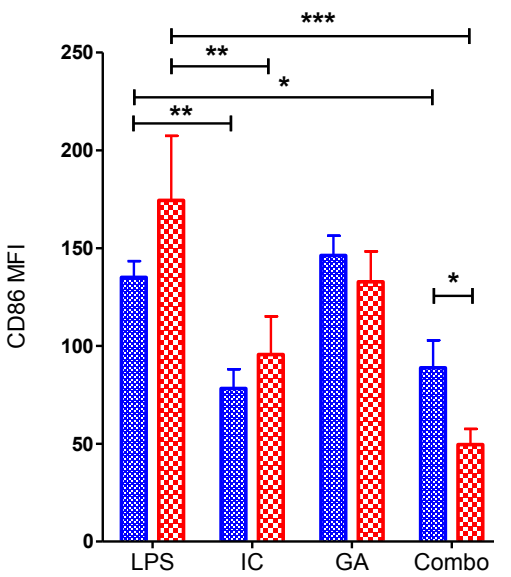

e.

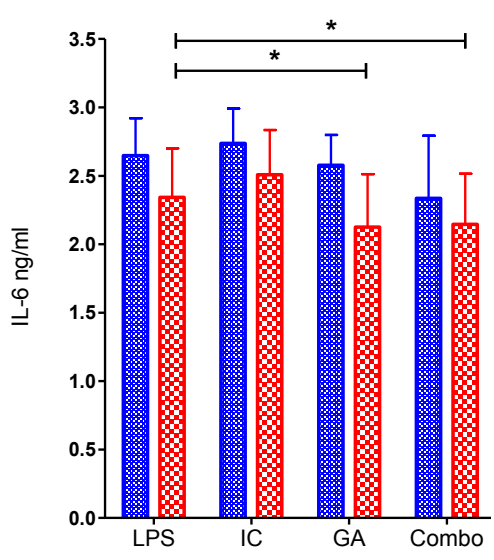

c.

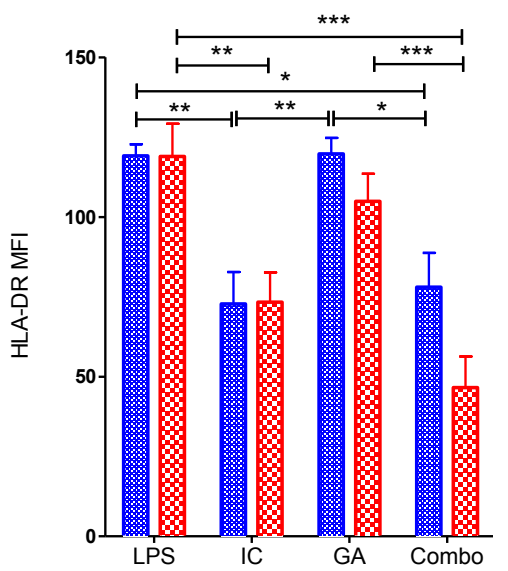

f.

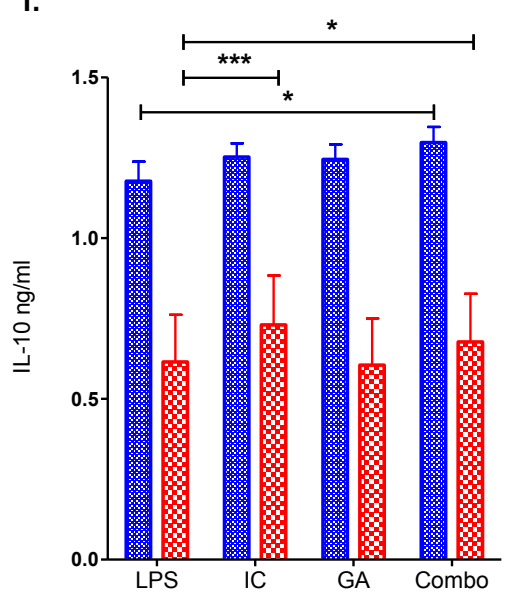

g.

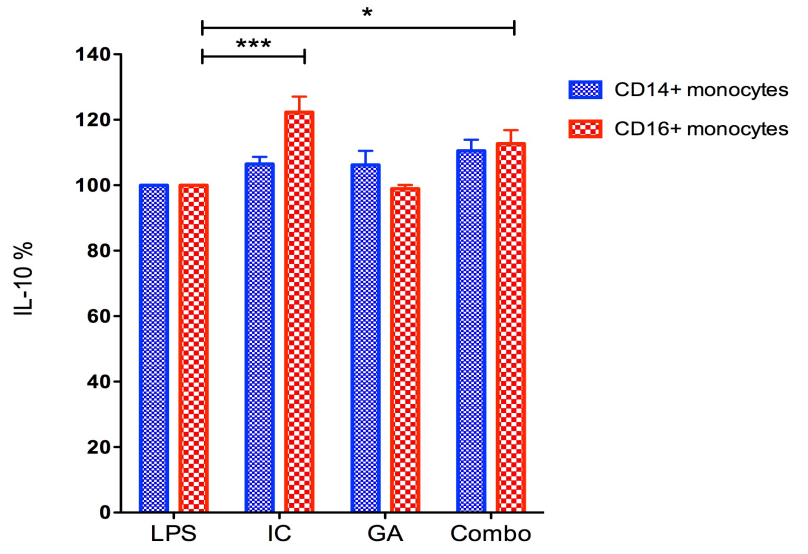

Figure 5.6. Type II activation of $\mathrm{CD}^{+} 4^{+}$and $\mathrm{CD}^{+}{ }^{+}$monocytes from MS patients.

$\mathrm{CD}_{14}{ }^{+}$and $\mathrm{CD}_{1} 6^{+}$monocytes from MS patients $(\mathrm{n}=4)$ were primed and treated with GA and/or IC in the presence of LPS for 24 hours. The subsets were stained with flow antibodies; the activation marker expression was assessed by flow cytometry and the cytokine production was measured by ELISA. (a) CD40. (b) CD86. (c) HLA-DR. (d) IL-12. (e) IL-6. (f) IL-10. (g) Production of IL-10 shown as a relative percentage to LPS. LPS group was normalized as $100 \%$. Shown are the means and SD of 4 individual experiments in MS group. The statistical analysing was done by using one way ANOVA with Dunnett's post-test to compare three or more groups and Student's t-test to compare 2 groups. $* \mathrm{P}<0.01 ; * * \mathrm{P}<0.005 ; * * * \mathrm{P}<0.001$. Data were collected on flow cytometry using the gating strategies in appendices 10,11 . 


\begin{tabular}{|l|llll|}
\hline Markers and cytokines & \multicolumn{2}{|l|}{ CD14+ Mo } & \multicolumn{2}{|l|}{ CD16+ Mo } \\
\hline CD40 & $60,8 \%$ & $( \pm 23)$ & $34,2 \%$ & $( \pm 9)$ \\
\hline CD86 & $67 \%$ & $( \pm 23)$ & $29,1 \%$ & $( \pm 7)$ \\
\hline HLA-DR & $65,6 \%$ & $( \pm 19)$ & $37,8 \%$ & $( \pm 12)$ \\
\hline IL-12 & $39,3 \%$ & $( \pm 16)$ & $62 \%$ & $( \pm 25)$ \\
\hline IL-6 & $86,1 \%$ & $( \pm 23)$ & $90,7 \%$ & $( \pm 7)$ \\
\hline IL-10 & $110,5 \%$ & $( \pm 7)$ & $112,7 \%$ & $( \pm 8)$ \\
\hline
\end{tabular}

Table 5.1. Type II activation of subsets by the combination treatment in MS patients.

$\mathrm{CD}_{1} 4^{+}$and $\mathrm{CD}_{1} 6^{+}$monocytes from MS patients $(\mathrm{n}=4)$ were primed and treated with GA and IC in the presence of LPS for 24 hours. The subsets were stained with antibodies, and the activation marker expression was assessed by flow cytometry. Cytokine production was measured by ELISA. The data is expressed as the relative expression compared to LPS treatment (\% expression) and shown are the means and SD of 4 individuals in MS group. Data were collected on flow cytometry using the gating strategies in appendices 10,11 . 


\subsubsection{The effect of subset interactions on the activation of $\mathrm{CD}^{+} 4^{+}$and CD16 $^{+}$monocytes}

All the above-mentioned results from our study are from the experiments in which the monocyte subsets have been separated and cultured individually. The individually cultured subset studies can give us important ideas about the function of individual CD $14^{+}$and $\mathrm{CD} 16^{+}$ monocytes in MS, and help us to prove the direct effects of GA and IC on the specific subsets. However, it is also crucial to understand that the subsets can interact with each other in blood and this interaction might change their immune response. In order to reveal how the interaction between the subsets affects the expressions of markers and cytokines in classically and type II activated monocytes in MS, the monocyte subsets were co-cultured in the presence or absence of LPS, GA and IC. The effect of the subsets' interaction on cytokine production was not assessed in this study due to the difference in the methods used to measure the cytokines in co-cultured versus individually cultured subsets. As such, in cocultured subsets, the level of cytokines was measured by flow cytometry, whereas in individually cultured subsets the method of ELISA was used. Therefore, this study only showed the impact of the subsets' interactions on the expression of markers in co-cultured $\mathrm{CD}_{14}{ }^{+}$and $\mathrm{CD}_{16}{ }^{+}$monocytes. While type II activation can be induced by GA and IC in isolated monocyte subsets, it is unclear how an interaction between the CD14 $4^{+}$and $\mathrm{CD} 16^{+}$ monocytes can affect the type II activation by GA and IC when cultured together. To address this question, the $\mathrm{CD} 14^{+}$and $\mathrm{CD}_{1} 6^{+}$monocytes were co-cultured as a total monocyte population, primed with IFN- $\gamma$ and treated with LPS, IC and/or GA. The expression of markers and intracellular cytokines was measured using flow cytometry to distinguish the individual subsets.

\subsubsection{Classical activation of co-cultured subsets}

When the classical activation of co-cultured subsets was compared to the basal state, both $\mathrm{CD} 14^{+}$and $\mathrm{CD} 16^{+}$monocytes from healthy and MS groups showed the characteristics of classical activation upon LPS stimulation. Specifically, the CD14 ${ }^{+}$monocytes had an increased expression of CD40, CD86, HLA-DR, IL-6 and IL-10 in both groups with a higher increase in the activation markers in MS group. When the expression of activation markers was compared between the co-cultured and individually cultured $\mathrm{CD} 14^{+}$monocytes, the 
expression of CD40 and HLA-DR was similar in both cultures, although the expression of CD86 was significantly lower in the co-cultured $\mathrm{CD} 14^{+}$monocytes from healthy subjects, than in individually cultured $\mathrm{CD} 14^{+}$monocytes. In the $\mathrm{CD} 14^{+}$monocytes from MS patients, however, the difference in the expression of markers was not significant (Figure 5.7).

While the $\mathrm{CD}_{16}{ }^{+}$monocytes from both healthy and MS groups had increased expression of CD40, IL-6 and IL-10, only CD16 ${ }^{+}$monocytes from MS group upregulated the expression of CD86 and HLA-DR upon the LPS stimulation. When the activation marker expression was compared between the co-cultured and individually cultured $\mathrm{CD}^{+} 6^{+}$monocytes, the expression of CD40 and CD86 was significantly lower in co-cultured CD16 ${ }^{+}$monocytes from healthy subjects, whereas the expression of HLA-DR was similar in both cultures. In MS patients, however, there was no difference in the expressions of markers between the cocultured and individually cultured $\mathrm{CD}_{1} 6^{+}$monocytes detected (Figure 5.8). Overall, although the interaction between the monocyte subsets inhibited the expression of activation markers in healthy subjects, $\mathrm{CD} 14^{+}$and $\mathrm{CD}_{1} 6^{+}$monocytes from both healthy and MS groups had characteristics of the classical activation after the LPS stimulation in co-cultures.

\subsubsection{The effect of GA on co-cultured subsets}

The co-cultured $\mathrm{CD}_{1} 4^{+}$and $\mathrm{CD} 16^{+}$monocytes from both groups had downregulated expression of CD40 and HLA-DR after GA treatment, whereas only subsets from healthy subjects had decreased expression of CD86 (Figures 5.9 and 5.10). The expression of IL-6 and IL-10 was not altered in either subsets from healthy or MS groups, except for slightly increased expression of IL-10 in CD16 ${ }^{+}$monocytes from MS patients (Figures 5.10.d,e). When the activation marker expression was compared between the co-cultured and individually cultured subsets, the expression levels of CD40 and HLA-DR were significantly lower in the co-cultured $\mathrm{CD} 14^{+}$monocytes from MS patients, but not from healthy subjects, after the GA treatment; whereas, the $\mathrm{CD} 16^{+}$monocytes did not show any significant differences. Therefore, GA-treated $\mathrm{CD}_{1} 4^{+}$and $\mathrm{CD} 16^{+}$monocytes did not have antagonistic effects. Instead, their interaction enhanced the type II activating effect of GA treatment on $\mathrm{CD} 14^{+}$monocytes in MS groups while maintaining the similar type II activating effect on $\mathrm{CD}_{16}{ }^{+}$monocytes in both groups, in comparison to the individually cultured subsets. 


\subsubsection{The effect of IC on co-cultured subsets}

The type II activation of co-cultured subsets by IC has been analyzed in comparison to the classically activated co-cultured subsets (positive control). Although the co-cultured CD14 ${ }^{+}$monocytes from both groups down-regulated the expression of CD40 after the treatment with IC, only subsets from MS patients had modestly decreased expression of CD86; however, the differences did not reach significance. Unlike CD14 ${ }^{+}$monocytes from healthy subjects, which had slightly increased expression of intracellular IL-10, the CD14 monocytes from MS patients had significantly decreased expression of IL-10. When the marker expressions were compared between the co-cultured and individually cultured subsets, the expressions of CD40 and CD86 were found to be lower in co-cultured CD14 ${ }^{+}$ monocytes from healthy subjects, but not from MS patients, after the IC treatment. While the co-cultured $\mathrm{CD} 16^{+}$monocytes from both groups had downregulated expression of CD40 and IL-10 after the treatment with IC, only subsets from healthy subjects had significantly decreased expression of CD86. The levels of HLA-DR and IL-6 were not altered by the IC treatment of either subset in both groups (Figures 5.9, 5.10). To conclude, although the interaction between $\mathrm{CD} 14^{+}$and $\mathrm{CD} 16^{+}$monocytes impairs the type II activating effect of IC in both healthy and MS groups, both subsets maintain a type II activated phenotypes when co-cultured.

\subsubsection{The effect of combination treatment on co-cultured subsets}

After GA and IC combination treatment, co-cultured healthy $\mathrm{CD} 14^{+}$and $\mathrm{CD} 16^{+}$ monocytes had reduced MFI expression of CD40 and HLA-DR and increased expression of IL-6 and IL-10 (Figure 5.9). Only healthy $\mathrm{CD}^{+} 6^{+}$monocytes had significantly downregulated expression of CD86, but not CD14 ${ }^{+}$monocytes. In MS patients, both cocultured subsets had decreased expression of CD40 and CD86 after the combination treatment, with no significant change in the expression of HLA-DR, IL-6 and IL-10 (Figure 5.10).

In order to assess the effect of combination treatment in each individual subject, the relative expression of activation markers in GA- and IC-treated monocytes to the expression in classically activated monocytes was analyzed (i.e. expressed as a percentage). The relative expression levels of CD40, CD86 and HLA-DR markers on MS CD14 ${ }^{+}$monocytes were similar in both individual and co-cultures (Table 5.2), whereby a decrease in the expression 


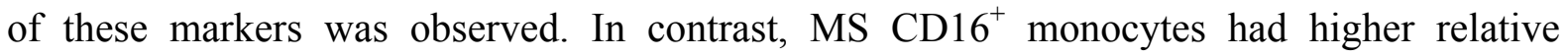
expression levels of the following markers in co-cultures: CD40 (90\% in co-cultures vs. 34\% in individual cultures), CD86 (77\% in co-cultures vs. $29 \%$ in individual cultures) and HLADR (99\% in co-cultures vs. $38 \%$ in individual cultures). Overall, these results suggest that, although the interaction between the subsets did not interfere with the type II activating effect of the combination treatment on MS CD14 $4^{+}$monocytes, it impaired the response of MS $\mathrm{CD} 6^{+}$monocytes to the combination treatment. This suggests that CD $14^{+}$monocytes regulate $\mathrm{CD}_{16}{ }^{+}$monocytes during GA and IC combination treatment, in MS patients.

When the effects of single and combination treatments were compared in co-cultured subsets, the co-inhibitory effect of $\mathrm{CD} 14^{+}$and $\mathrm{CD} 16^{+}$monocytes was variable between the different treatment groups. Although the type II activating effect of single GA treatment was equal or enhanced in interacting $\mathrm{CD}_{1} 4^{+}$and $\mathrm{CD} 16^{+}$monocytes, the effect of single IC treatment was impaired in interacting $\mathrm{CD} 14^{+}$and $\mathrm{CD}_{1} 6^{+}$monocytes from MS patients. However, the GA and IC combination treatment improved the type II activating effect of IC treatment alone, on co-cultured $\mathrm{CD}_{1} 4^{+}$monocytes (Table 5.3). In conclusion, this study demonstrated, for the first time, that an interaction between $\mathrm{CD}_{1} 4^{+}$and $\mathrm{CD}_{1} 6^{+}$monocytes modulated their activation after the treatments with GA and IC in MS patients. 

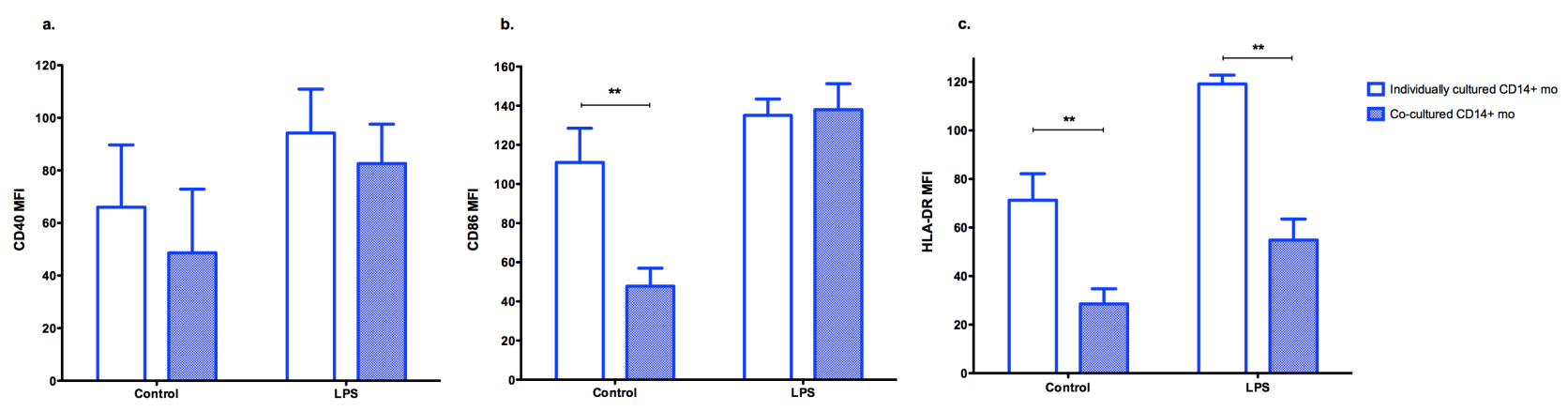

Figure 5.7. Classical activation of individually cultured and co-cultured CD14 ${ }^{+}$monocytes from MS patients.

$\mathrm{CD}_{1} 4^{+}$monocytes from MS patients $(\mathrm{n}=20)$ were cultured in the absence or presence of CD16 monocytes (as a total monocyte culture), primed with $20 \mathrm{U} / \mathrm{ml}$ IFN- $\gamma$ overnight and stimulated with $200 \mathrm{ng} / \mathrm{ml}$ LPS for 24 hours. The monocytes were then harvested and stained with antibodies. The activation marker expression on CD14 monocytes was assessed by flow cytometry. The control group represents unstimulated and untreated monocytes. (a-c) The expressions of markers and cytokines in CD14 monocytes: (a) CD40, (b) CD86, (c) HLA-DR. Shown are the means and SD from individuals in the MS group. The statistical analysing was done by using Student's t-test. ${ }^{* *} p<0.005$. Data were collected on flow cytometry using the gating strategies in appendices 9,10 .
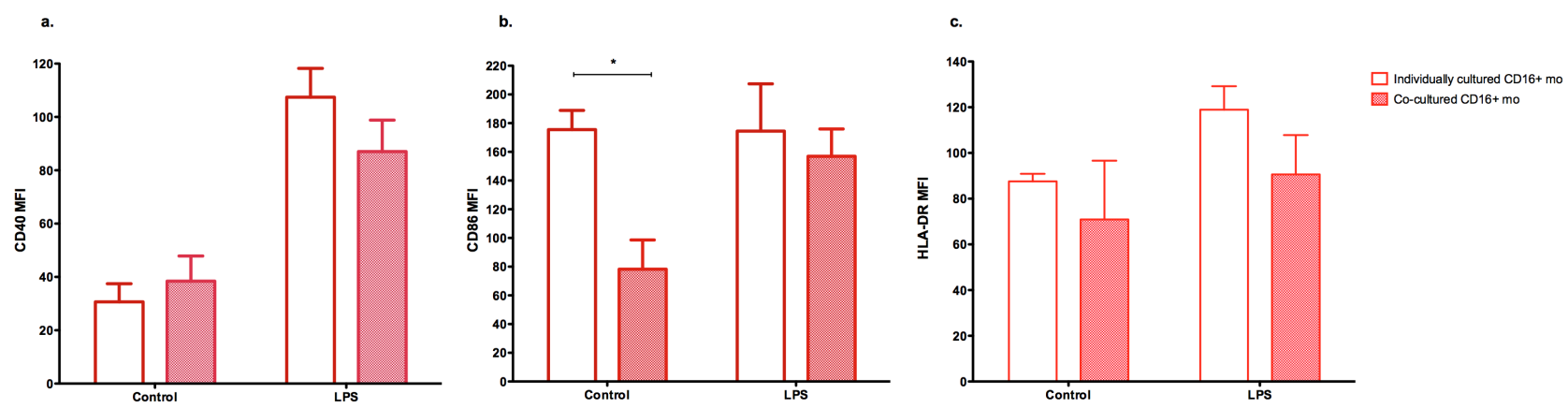

Figure 5.8. Classical activation of individually cultured and co-cultured $\mathrm{CD}^{+}$monocytes from MS patients.

$\mathrm{CD}_{16} 6^{+}$monocytes from MS patients $(\mathrm{n}=20)$ were cultured in the absence or presence of CD14 $4^{+}$monocytes (as a total monocyte culture), primed with $20 \mathrm{U} / \mathrm{ml}$ IFN- $\gamma$ overnight and stimulated with $200 \mathrm{ng} / \mathrm{ml}$ LPS for 24 hours. The monocytes were then harvested and stained with antibodies. The activation marker expression on CD16 ${ }^{+}$ monocytes was assessed by flow cytometry. The control group represents unstimulated and untreated monocytes. (a-c) The expressions of markers and cytokines in CD16 ${ }^{+}$monocytes: (a) CD40, (b) CD86, (c) HLA-DR. Shown are the means and SD from individuals in the MS group. The statistical analysing was done by using Student's t-test. *p $<0.001$. Data were collected on flow cytometry using the gating strategies in appendices 9,11 . 

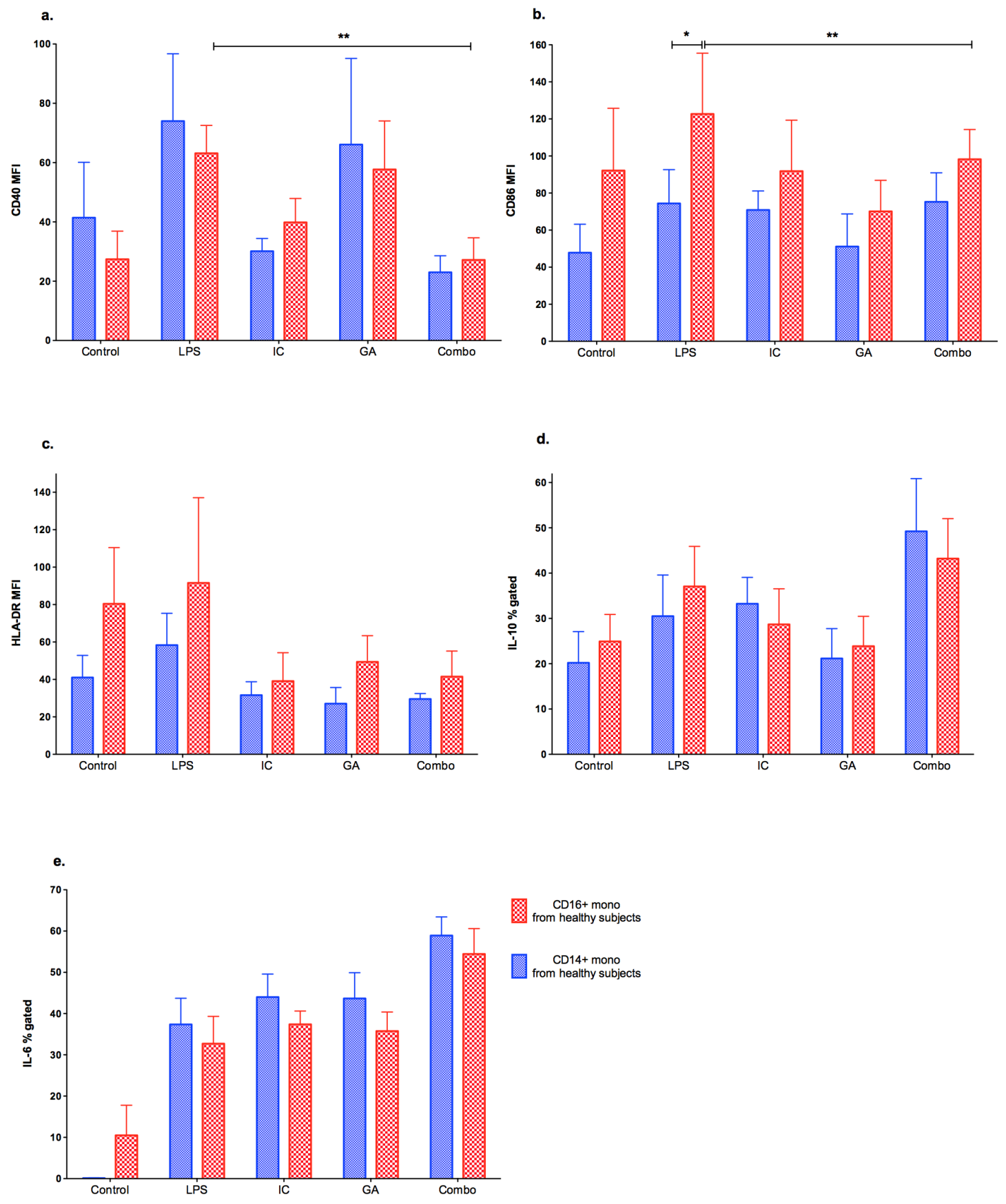

Figure 5.9. Type II activation of co-cultured $\mathrm{CD}^{+} 4^{+}$and $\mathrm{CD}^{+}{ }^{+}$monocytes from healthy subjects.

$\mathrm{CD}_{1} 4^{+}$and $\mathrm{CD}_{1} 6^{+}$monocytes from healthy subjects $(\mathrm{n}=20)$ were co-cultured, primed and treated with GA and/or IC in the presence of LPS for 24 hours. The monocytes were stained with antibodies, and the activation marker expression was assessed by flow cytometry. The cytokine production was measured by intracellular staining and flow cytometry. The control group represents unstimulated and untreated monocytes. (a-e) The expression of markers and cytokines in healthy subsets: (a) CD40, (b) CD86, (c) HLA-DR, (d) IL-10, (e) IL-6. Shown are the means of MFI or percent gated populations and SD of 20 individuals in the healthy group. The statistical analysing was done by using one way ANOVA to compare three or more groups and Student's t-test to compare 2 groups. ${ }^{*} p<0.01 ; * p<0.005$. Data were collected on flow cytometry using the gating strategy in appendix 9. 

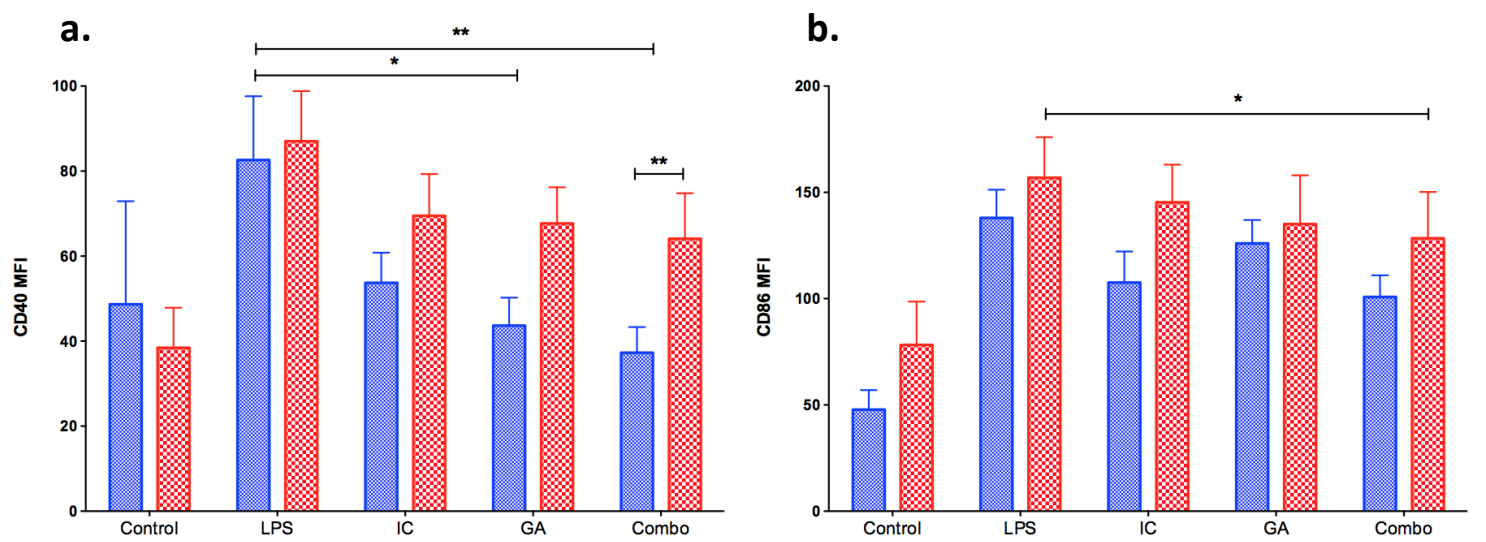

c.
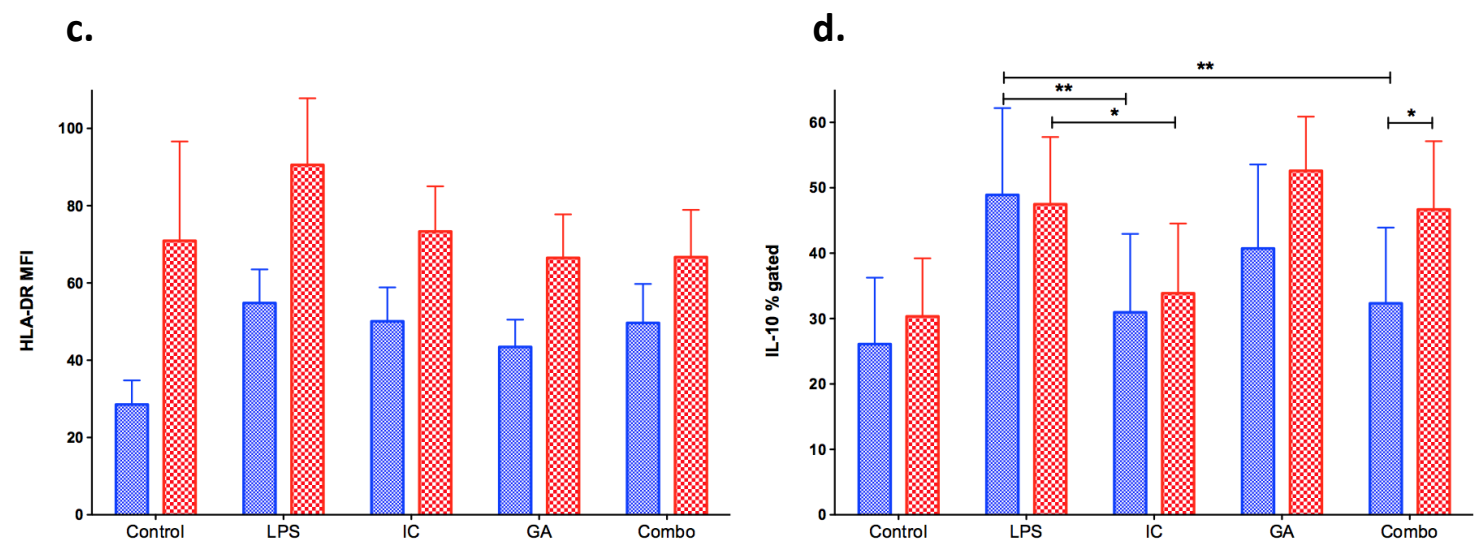

e.

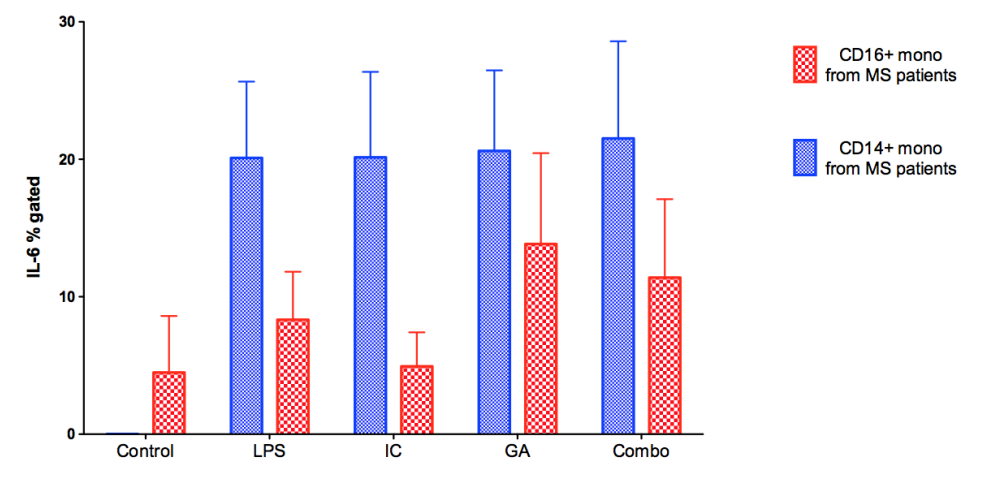

Figure 5.10. Type II activation of co-cultured $\mathrm{CD}_{14}{ }^{+}$and $\mathrm{CD} 16^{+}$monocytes from MS patients.

$\mathrm{CD}_{14}{ }^{+}$and $\mathrm{CD} 16^{+}$monocytes from MS patients ( $\mathrm{n}=20$ ) were co-cultured, primed and treated with $\mathrm{GA}$ and/or IC in the presence of LPS for 24 hours. The monocytes were stained with antibodies, and the activation marker expression was assessed by flow cytometry. The cytokine production was measured by intracellular staining and flow cytometry. The control group represents unstimulated and untreated monocytes. (a-e) The expression of markers and cytokines in MS patients: (a) CD40, (b) CD86, (c) HLA-DR, (d) IL-10, (e) IL-6. Shown are the means of MFI or percent gated populations and SD of 20 individuals in the MS group. The statistical analysing was done by using one way ANOVA to compare three or more groups and Student's t-test to compare 2 groups. $* \mathrm{p}<0.01 ; * * \mathrm{p}<0.005$. Data were collected on flow cytometry using the gating strategy in appendix 9 . 


\begin{tabular}{|l|l|ll|l|ll|}
\hline \multicolumn{4}{|l|}{ CD14+ Mo } & \multicolumn{2}{l|}{ CD16+ Mo } \\
\hline Cultures: & Individual & \multicolumn{2}{l|}{ Co-cultures } & Individual & \multicolumn{2}{l|}{ Co-cultures } \\
\hline CD40 & $60,8( \pm 23)$ & $55,5 \%$ & $( \pm 23)$ & $34,2( \pm 9)$ & $90,3 \%$ & $( \pm 73)$ \\
\hline CD86 & $67( \pm 23)$ & $64,4 \%$ & $( \pm 39)$ & $29,1( \pm 7)$ & $76,6 \%$ & $( \pm 55)$ \\
\hline HLA-DR & $65,6( \pm 19)$ & $80,8 \%$ & $( \pm 49)$ & $37,8( \pm 11,7)$ & $98,9 \%$ & $( \pm 64)$ \\
\hline
\end{tabular}

Table 5.2. Relative expression of activation markers after the GA and IC combination treatment of $\mathrm{CD}^{+}{ }^{+}$and $\mathrm{CD} 16^{+}$monocytes from MS patients.

$\mathrm{CD} 14^{+}$and $\mathrm{CD} 16^{+}$monocytes from MS patients $(\mathrm{n}=20)$ were either individually or co-cultured, primed and treated with GA and IC in the presence of LPS for 24 hours. The monocytes were stained with antibodies, and the activation marker expression was assessed by flow cytometry. The expression of markers is shown as a relative expression to LPS treatment (\% expression). The LPS treatment was normalized to $100 \%$. Shown are the means $( \pm \mathrm{SD})$. Data were collected on flow cytometry using the gating strategies in appendices 9-11.

\begin{tabular}{|c|c|c|c|c|c|c|}
\hline \multirow{2}{*}{ Treatment } & \multicolumn{2}{|l|}{ CD40 } & \multicolumn{2}{|l|}{ CD86 } & \multicolumn{2}{|l|}{ HLA-DR } \\
\hline & CD14+ Mo & CD16+ Mo & CD14+ Mo & CD16+ Mo & CD14+ Mo & CD16+ Mo \\
\hline $\mathrm{IC}$ & $77,6 \% \quad( \pm 24)$ & $93,6 \% \quad( \pm 60)$ & $108,8 \%( \pm 81)$ & $86,7 \% \quad( \pm 35)$ & $\begin{array}{ll}95 \% & ( \pm 52)\end{array}$ & $100,6 \%( \pm 62)$ \\
\hline GA & $62,5 \% \quad( \pm 25)$ & $91,8 \% \quad( \pm 63)$ & $104 \% \quad( \pm 94)$ & $69,9 \% \quad( \pm 41)$ & $88,8 \% \quad( \pm 40)$ & $90,4 \% \quad( \pm 54)$ \\
\hline Combo & $55,5 \% \quad( \pm 23)$ & $90,3 \% \quad( \pm 73)$ & $64,4 \% \quad( \pm 39)$ & $76,6 \% \quad( \pm 55)$ & $80,8 \% \quad( \pm 49)$ & $98,9 \% \quad( \pm 64)$ \\
\hline
\end{tabular}

Table 5.3. Type II activation of co-cultured subsets from MS patients.

$\mathrm{CD}_{14}{ }^{+}$and $\mathrm{CD} 16^{+}$monocytes from MS patients ( $\left.\mathrm{n}=20\right)$ were co-cultured, primed and treated with GA and IC in the presence of LPS for 24 hours. The monocytes were stained with antibodies, and the activation marker expression was assessed by flow cytometry. The expression of markers is shown as a relative expression to LPS treatment (\% expression). The LPS treatment was normalized to $100 \%$. Shown are the means $( \pm \mathrm{SD})$. Data were collected on flow cytometry using the gating strategy in appendix 9 . 


\subsection{DISCUSSION}

\subsubsection{Classical activation of $\mathrm{CD}_{14}^{+}$and $\mathrm{CD}^{+}{ }^{+}$monocytes in vitro}

MS CD $14^{+}$and $\mathrm{CD}_{1} 6^{+}$monocytes have been shown to be active inflammatory cells via their increased basal expressions of cell surface markers and production of cytokines. These basal levels are likely due to the disease process and not related to the method of isolation and culture, as the $\mathrm{CD} 14^{+}$monocytes in the control healthy group only had minimal levels of these markers and did not produce any detectable levels of cytokines. Despite being already active, an additional in vitro stimulation with LPS significantly enhanced the inflammatory phenotypes of the $\mathrm{CD} 14^{+}$and $\mathrm{CD} 16^{+}$monocytes of MS patients.

The in vitro classical activation of inflammatory $\mathrm{CD}^{+} 4^{+}$monocytes of MS patients appeared to be mainly via the induction of cytokine production, rather than through a change in surface marker expressions. As the basal level of the markers was already significantly high in the patients' untreated monocytes, the classical activation of MS CD14 ${ }^{+}$monocytes might be a multi-step process. Unlike healthy monocytes, the production of markers and cytokines might not necessarily occur at the same time in MS CD14 $4^{+}$monocytes. As such, it is possible that an induction in the expression levels of inflammatory markers occurs during the initial step of the classical activation of $\mathrm{CD} 14^{+}$monocytes during MS. The subsequent stage could involve the active production of cytokines with further inductions of the activation markers. Alternatively, it is possible that the inflammatory cytokine secretion is not a continuous process. Hence, the initial pulse of the cytokines could have occurred earlier, followed by a delayin the production of new cytokines.

Although the levels of HLA-DR and IL-6 were significantly higher in the in vitro, classically activated $\mathrm{CD} 14^{+}$monocytes from MS patients, the expression levels of CD40 and CD86 were not different to $\mathrm{CD} 14^{+}$monocytes from healthy patients. The classical activation of this subset led to the upregulation of HLA-DR, IL-6, IL-12 and IL-10. However, the levels of CD40 and CD86 were not significantly different from that of un-stimulated CD14 monocytes from MS patients. Overall, this work suggests that the in vitro classically activated $\mathrm{CD} 14^{+}$monocytes from MS patients have a better capacity for antigen presentation and activation of $\mathrm{T}$ cells compared to the monocytes from healthy patients. However, the $\mathrm{CD}_{14}{ }^{+}$monocytes did not seem to be the main co-stimulators of Th1 cells in MS patients. 
The in vitro, classically activated $\mathrm{CD} 16^{+}$monocytes from MS patients had higher levels of HLA-DR, IL-6 and IL-10, suggesting that they were more effective inflammatory cells compared to the $\mathrm{CD} 16^{+}$monocytes from healthy patients. Unlike $\mathrm{CD} 14^{+}$monocytes from MS patients, the classical activation of $\mathrm{CD}^{+} 6^{+}$monocytes from $\mathrm{MS}$ patients resulted in significantly increased levels of HLA-DR and cytokines, as well as inducing the expression of CD40. This indicates that the $\mathrm{CD}^{+} 6^{+}$monocytes in MS patients might be important stimulators of Th1 and Th17 cells in MS.

Although the extracellular levels of IL- 6 and IL-10 were significantly higher in CD14 ${ }^{+}$ and $\mathrm{CD}^{+} 6^{+}$from MS patients (compared to monocytes from healthy subjects), the intracellular levels were not. This indicates that the in vitro classical activation of monocyte subsets from MS patients did not induce a renewed production of IL-6 and IL-10 at the timepoint tested, but rather enhanced the release of produced cytokines from the cells. The IL-6[210] and IL-10[211] receptors were detected on monocytes and it has been shown previously that monocytes can re-uptake the released cytokines and auto-regulate their productions[200, 210]. As such, the increased extracellular amount of these cytokines could trigger a negative feedback response to inhibit the production of IL-6 and IL-10 that results from the classical activation. Therefore, it is possible that at earlier timepoints a similar pattern of intracellular cytokine production might be seen.

Classical activation, as well as the treatments with GA and IC, did not alter the expression levels of CCR2 and CD64 in monocyte subsets, in either patients groups. It has been shown that classically activated monocytes from healthy patients exhibit downregulated expression of CCR2[212]. The MS monocyte subsets in this study did not exhibit the expected down-regulated expression of CCR2, indicating that they might sustain their ability to migrate in the response to CCL2 chemokine, regardless of the activation state. Similarly, the high level of CD64 in both un-stimulated and stimulated subsets indicate that the $\mathrm{CD} 14^{+}$and $\mathrm{CD} 16^{+}$monocytes might have a similar ability to bind immune complexes when activated.

Although both MS monocyte subsets were classically activated by the LPS treatment, the classically activated $\mathrm{CD} 14^{+}$and $\mathrm{CD} 16^{+}$monocytes exhibited differences in their phenotypes. Our results showed that classically activated $\mathrm{CD} 14^{+}$monocytes produced significantly higher amounts of IL-10 compared to $\mathrm{CD} 16^{+}$monocytes, in healthy subjects. This corresponds to the findings of Mizuno et al., who observed decreased levels of the anti-inflammatory IL-10 cytokine, in classically activated $\mathrm{CD} 16^{+}$monocytes[81]. Interestingly, in MS patients, CD14 monocytes also appeared to have a more regulatory phenotype compared to the $\mathrm{CD} 16^{+}$ 
monocytes, and are better producers of IL-10. No previous study has characterized the activation of MS monocyte subsets in this detail. This study shows for the first time that, among the monocyte subsets, $\mathrm{CD}^{+} 6^{+}$monocytes are highly inflammatory and have a decreased ability to produce anti-inflammatory cytokines, in MS patients. To conclude, both $\mathrm{CD}_{14}{ }^{+}$and $\mathrm{CD}_{16}{ }^{+}$monocytes during MS became classically activated by in vitro LPS stimulation and showed the characteristics of pro-inflammatory cells. Furthermore, the $\mathrm{CD}_{1} 6^{+}$monocytes appeared to be more active drivers of inflammation than the $\mathrm{CD} 14^{+}$ monocytes in MS patients.

\subsubsection{The effect of $\mathrm{GA}$ on $\mathrm{CD}^{+} 4^{+}$and $\mathrm{CD}^{+}{ }^{+}$monocytes in vitro}

GA directly affects both drivers of inflammation in MS patients, such as T cells and monocytes, and induces an anti-inflammatory phenotype in these cells. Moreover, GA-treated monocytes can specifically inhibit the proliferation of inflammatory $\mathrm{T}$ cells in mouse model of MS, independently of other regulatory cells, such as Tregs[43]. Monocytes are not only actively involved in the disease course, but they also can regulate other inflammatory cells in MS; hence, they are one of the most important and attractive targets of GA during the treatment of MS. This study shows that GA has a direct type II activating effect on CD14 and $\mathrm{CD} 16^{+}$monocytes from MS patients and healthy subjects.

Besides targeting inflammatory Th1 cells in MS, GA has been shown to have a direct effect on monocytes[21]. GA uptake is mediated through HLA-DR[164] and $\alpha_{M} \beta_{2}$ integrins[213]. However, its effect does not seem to be solely dependent on HLA-DR binding, as GA has been effective on monocytes in an HLA-DR-deficient mouse model of MS[166]. Furthermore, more than 95\% of mouse monocytes bind GA within 3-6 hours following GA injection and half of them are CD115 ${ }^{+} \mathrm{Ly}-6 \mathrm{C}^{+}$cells[41] the counterparts of human $\mathrm{CD}_{1} 4^{+}$monocytes[214]. This indicates that the $\mathrm{GA}^{+}$monocytes (comprising $95 \%$ of mouse monocytes) consist of both subsets, suggesting that GA can effectively bind to both subsets of monocytes in the mouse model of MS.

GA can type II activate total monocyte populations and inhibit CD40, CD86 and IL-12 levels in MS patients (Chapter 4). In agreement with our study, Kim et al. have reported that human monocytes, type II activated by GA, attain the ability to inhibit inflammatory Th1 cells in MS[21]. As it is unclear whether GA can affect both human monocyte subsets, this 
study has further looked at the GA effect on human $\mathrm{CD} 14^{+}$and $\mathrm{CD} 16^{+}$monocytes that have been cultured individually.

Our study has revealed that human monocyte subsets respond differently to the in vitro GA treatment in MS patients. Only the down-regulation of CD64 and IL-12 was observed in $\mathrm{CD}_{14}{ }^{+}$subsets from MS patients. Thus, the effect of GA on individually cultured CD14 ${ }^{+}$ monocytes was mostly related to the regulation of inflammatory cytokines (i.e. IL-12), but not co-stimulatory markers in MS. On the other hand, GA had a better effect on CD16 ${ }^{+}$ monocytes from MS patients that had reduced levels of the markers CD40, CD86 and CD64, as well as the inflammatory cytokines IL-6 and IL-12, after the treatment. This work indicates that $\mathrm{CD} 16^{+}$monocytes, that have been type II activated by GA, can inhibit the activation of Th1 and Th17 cells through the regulation of co-stimulatory pathways and T cell attraction to the inflammatory site. These findings also suggest that the GA treatment of individual monocyte subsets leads to the type II activation of both $\mathrm{CD} 14^{+}$and $\mathrm{CD} 16^{+}$monocytes in MS patients, with a more prominent effect on the $\mathrm{CD} 16^{+}$monocytes. Overall, this study showed that, while affecting only cytokine production in $\mathrm{CD} 14^{+}$monocytes, GA affected both activation markers and cytokine expression levels in $\mathrm{CD} 16^{+}$monocytes, of MS patients.

As it is was shown previously, MS monocyte subsets bear phenotypical variations when classically activated. When treated with GA, the MS monocyte subsets maintained the variations in the productions of IL-6, IL-10 and IL-12 cytokines. As such, CD14 ${ }^{+}$monocytes remained the main producers of IL- 6 and IL-10, whereas CD $16^{+}$monocytes sustained a more active production of IL-12 after the treatment. Surprisingly, GA-treated CD14 $4^{+}$and CD16 ${ }^{+}$ monocytes from MS patients had similar levels of CD40 and CD86, despite the high expressions on the classically activated $\mathrm{CD}^{+} 6^{+}$monocytes. This indicates that GA reduced the capacity of $\mathrm{CD}^{+} 6^{+}$monocytes to co-stimulate inflammatory $\mathrm{T}$ cells, and the observed reduction was to the same low level as in the GA-treated CD14 $4^{+}$monocytes of MS patients. Overall our study shows for the first time that GA has direct type II activating effects on individual $\mathrm{CD}_{1} 4^{+}$and $\mathrm{CD} 16^{+}$monocyte subsets from MS patients and healthy subjects. Unlike with the type II activation by IC, in vitro GA treatment diminished the differences in the co-stimulatory abilities between $\mathrm{CD} 14^{+}$and $\mathrm{CD} 16^{+}$monocytes of MS patients. 


\subsubsection{The effect of $\mathrm{IC}$ on $\mathrm{CD}^{+} 4^{+}$and $\mathrm{CD}_{16}{ }^{+}$monocytes in vitro}

This study demonstrated that IC had a type II activating effect on both CD14 ${ }^{+}$and CD16 $6^{+}$ monocytes. In vitro IC treatment was used in this study as a model for in vivo intravenous immunoglobulin G (IVIG) treatment. IVIG is known to have an immunomodulatory effect and is successfully used for the treatment of autoimmune diseases[215]. Once injected, IVIG may bind antigens, such as human red blood cells, and form IC[174-176]. In order to better simulate the in vivo effect of IVIG, we treated the isolated blood monocytes with IC that consisted of human red blood cells coated with IgG.

Bayry et al. have observed an immunomodulatory effect of IVIG on healthy monocytederived dendritic cells in vitro[150]. They also found that IVIG inhibits the expression of cell surface markers, such as CD40, CD86 and HLA-DR, as well as the production of inflammatory IL-12 cytokine[150]. Although there was no significant change in IL-12 production in our study, we observed a similar inhibition of CD40, CD86 and HLA-DR expression by $\mathrm{IC}$, in both $\mathrm{CD}_{1} 4^{+}$and $\mathrm{CD} 16^{+}$monocyte subsets of MS patients. The difference in IL-12 production might be due to the fact that the dendritic cells, used by Bayry et al., are a more mature form of monocytes and express IL-12 receptor. Human monocytes, on the other hand, do not express IL-12 receptor[216]. Therefore, the LPS-induced production of IL-12 may inhibit the new IL-12 production in dendritic cells. It is possible that the autoregulation of IL-12 production in dendritic cells underlies the inhibition of IL-12 production observed by Bayry et al. in response to IVIG treatment[150]. An additional point of difference is that in our study IVIG bound to RBC was used, not free soluble IVIG as in the study of Bayry et al. Thus, the difference in IL-12 production could be due to modifications of different pathways by free IVIG and IC treatments.

In this study IC affected inflammatory marker expression, but not cytokine production by both $\mathrm{CD} 14^{+}$and $\mathrm{CD} 16^{+}$monocytes. This finding suggests that the type II activating effect of IC is limited to the inhibition of the ability of inflammatory monocytes to co-stimulate Th1 cells, but does not affect the ability to attract and activate Th1 cells by releasing inflammatory cytokines. Interestingly, in response to IC, a different effect was observed on the production of the anti-inflammatory IL-10 cytokine by the different subsets. Although IC-treated CD14 ${ }^{+}$ monocytes from MS patients produced only slightly increased levels of IL-10, compared to classically activated monocytes, $\mathrm{CD}_{1} 6^{+}$monocytes from MS patients had a significant increase in IL-10 secretion after IC treatment. However, the elevated secretion of IL-10, as 
measured extracellularly, was not supported by intracellular IL-10 production. The intracellular staining of IL-10 revealed a decrease in not only the amount of IL-10-expressing cells, but also a lower production of IL-10 in individual cells. Monocytes express the IL-10 receptor[211] and can thus autoregulate IL-10 production[217]. The increased extracellular IL-10 could result from a negative feedback loop, which inhibits the new production of this cytokine. Interestingly reduced intracellular IL-10 was observed in the monocyte subsets, isolated from MS patients only, whereas IC-treated monocytes from healthy subjects maintained a similar or higher level of intracellular IL-10. This reduced synthesis of IL-10 by MS subsets compared to LPS was likely due to the more inflammatory nature of the subsets in MS patients compared to healthy people. Alternatively, it is worth mentioning that the intracellular cytokine staining was done in co-cultured monocyte subsets where the CD14 ${ }^{+}$ and $\mathrm{CD}_{16} 6^{+}$monocytes can interact with each other. Therefore, another reason for the reduced production of intracellular IL-10 may be due to an interaction between inflammatory CD14 ${ }^{+}$ and $\mathrm{CD} 16^{+}$monocytes. On the other hand, the intracellular cytokine production was assessed on monocytes from only 7-8 subjects, and a large degree of variability between the subjects was observed. These factors may limit the sensitivity of our assays, only allowing the detection of large changes in the cytokine production, and limiting the detection of any modest changes. Overall, despite the inflammatory nature of MS subsets, the individually cultured $\mathrm{CD}_{1} 4^{+}$and $\mathrm{CD} 16^{+}$monocytes of MS patients had the basic phenotype of type II activated monocytes after IC treatment.

As shown previously in this study, classically activated monocyte subsets express a specific pattern of cytokines and surface markers. Interestingly, the differences between the subsets were still prominent after IC treatment and the difference in the levels of these between $\mathrm{CD}_{14}{ }^{+}$and $\mathrm{CD}_{1} 6^{+}$monocytes in MS patients, were not altered. Thus, in MS patients, $\mathrm{CD} 14^{+}$monocytes were the main producers of IL-6 and IL-10; whereas, CD16 ${ }^{+}$ monocytes maintained higher expression levels of IL-12, CD40 and CD86. These findings indicate that the type II activation of MS monocyte subsets by IC does not necessarily adjust the levels of markers and cytokines of each subset to the same level, but instead modifies the specific characteristics individually.

In MS patients, despite the fact that $\mathrm{CD}^{+} 6^{+}$monocytes had a more inflammatory phenotype compared to $\mathrm{CD} 14^{+}$monocytes, IC treatment directly targeted the co-stimulatory and antigen presentation pathways of both monocyte subsets, and triggered their type II activation. This study shows for the first time that in vitro IC treatment of individual CD $14^{+}$ 
and $\mathrm{CD}^{+} 6^{+}$monocyte subsets of MS patients resulted in their type II activation, while maintaining their phenotypical identities.

\subsubsection{The effect of GA and IC combination treatment on monocyte subsets in vitro}

Combination therapy has been considered as the most rational and efficient modality for the treatment of MS. It is postulated that multitherapy with two agents does not necessarily increase the side effects. Instead, it can prolong and improve the efficacy of the treatments, especially for those patients who do not respond to monotherapies[208]. Although many treatment combinations have been tried with little success[208], the combination of GA and IC has not been studied so far.

In this study, the GA and IC combination treatment has been shown to have a type II activating effect on $\mathrm{CD}_{1} 4^{+}$and $\mathrm{CD} 16^{+}$monocyte subsets, while maintaining the effects of both GA and IC single treatments. This study demonstrated that single IC treatment affected the marker expression levels in individual $\mathrm{CD} 14^{+}$and $\mathrm{CD} 16^{+}$monocyte subsets of $\mathrm{MS}$ patients. In contrast, single GA treatment altered both marker and cytokine levels in CD16 ${ }^{+}$ monocytes, and only cytokine productions in $\mathrm{CD}_{1} 4^{+}$monocytes. Additionally, our study found that the in vitro combination treatment with GA and IC had a better type II activating effect on individual $\mathrm{CD} 14^{+}$and $\mathrm{CD} 16^{+}$monocytes, similar to the observations from an earlier study looking at total monocyte populations (Chapter 4).

Interestingly, the response of the subsets to the combination treatment included the effects of both GA and IC single treatments, on $\mathrm{CD} 14^{+}$and $\mathrm{CD} 16^{+}$monocytes in MS. Thus, both $\mathrm{MS} \mathrm{CD} 14^{+}$and $\mathrm{CD}_{16}{ }^{+}$monocytes had down-regulated levels of CD40, CD86, HLA-DR, IL-12 and IL-6 after the in vitro combination treatment. Although the single treatments with GA and IC did not have a significant effect on the anti-inflammatory IL-10 cytokine production in $\mathrm{CD}_{1} 4^{+}$monocytes from MS patients, the combination treatment significantly up-regulated the production of IL-10 in both $\mathrm{CD} 14^{+}$and $\mathrm{CD} 16^{+}$monocytes from MS patients. This indicates that the combination treatment involves both GA and IC effects on the CD14 ${ }^{+}$ and $\mathrm{CD} 16^{+}$monocytes of MS patients, and the combination of these two treatments results in a better type II activation of the MS subsets than the single treatments.

Although both subsets became type II activated in response to the combination treatment, the cytokine productions were affected to different degrees in the two subsets. Specifically, in 
MS patients, CD16 ${ }^{+}$monocytes had significantly lower levels of IL- 6 compared to CD14 ${ }^{+}$ monocytes, after the combination treatment. This alteration is not significant in the total monocyte populations or in the $\mathrm{CD} 14^{+}$subset, indicating that the effect of the combination treatment on IL-6 production is most prominent in the CD16 ${ }^{+}$monocytes of MS patients. Moreover, in MS patients, the greater response of $\mathrm{CD} 16^{+}$monocytes was also evident from the 2-fold decrease in CD40, CD86 and HLA-DR marker expressions after the combination treatment, in comparison to the $\mathrm{CD} 14^{+}$monocytes. Therefore, the combination treatment might be a better approach for the treatment of MS, in order to target the highly inflammatory population of monocytes, such as the $\mathrm{CD}_{1} 6^{+}$subset.

Overall, this is the first study demonstrated that, in vitro, GA and IC combination treatment of inflammatory monocytes had a better type II activating effect on the individual $\mathrm{CD}_{1} 4^{+}$and $\mathrm{CD} 16^{+}$monocytes, than either agent alone. GA and IC have been shown to have synergistic effects and also to maintain the individual effects of both agents. This indicates that the co-treatment with GA and IC might be a better approach for the treatment of MS.

\subsubsection{Interaction between the subsets: response to the treatments in co- cultures}

Interactions between the subsets may significantly alter their response to various treatments. Our study demonstrates that, although an interaction between the monocyte subsets impaired the type II activating effect of IC on $\mathrm{CD}_{1} 4^{+}$and $\mathrm{CD} 16^{+}$monocytes, the GA and combination treatments had enhanced or equal effects on co-cultured subsets, compare to individual cultures. This may be due to the different pathways being engaged by these treatments. Mizuno et al. suggested that $\mathrm{CD} 14^{+}$and $\mathrm{CD}_{1} 6^{+}$monocytes might interact with each other through a paracrine mechanism[81]. However, the effect of this type of interaction on the function of human monocyte subsets has not yet been investigated. Both monocyte subsets express the IL-10 receptor (IL-10R)[211], and high level of this receptor can negatively affect new IL-10 production[217]. In our study, IC treatment modestly increased extracellular IL-10 production in both individual subsets, in comparison to classically activated subsets. It is possible that the increased level of IL-10 in the IC-treated subsets negatively affected the production of this regulatory cytokine, resulting in reduced production of IL-10 in interacting monocytes. This may help to explain the impaired type II activation of the subsets by IC that was observed when the subsets were co-cultured. 
Although GA acts through binding to the HLA-DR molecule on monocytes, this does not seem to be the only receptor for GA, as HLA-deficient mouse monocytes also respond to GA[166]. In our study, the type II activation in GA-treated $\mathrm{CD} 14^{+}$monocytes was enhanced by the interaction of the subsets, whereas GA-treated $\mathrm{CD}^{+} 6^{+}$monocytes had higher production of IL-10 cytokine in co-cultured MS subsets. Moniuszko et al. have shown that $\mathrm{CD}^{+} 6^{+}$monocytes express higher levels of IL-10R compared to CD14 ${ }^{+}$monocytes[211]. However, IL-10R is significantly downregulated by glucocorticoid treatment in only CD16 ${ }^{+}$ monocytes[211], possibly because of a higher chance to target IL-10R in CD16 ${ }^{+}$monocytes. Therefore, the augmented type II activation of $\mathrm{CD} 14^{+}$monocytes that was observed when these cells were allowed to interact with $\mathrm{CD} 16^{+}$monocytes, may be due to a GA-dependent downregulation of IL-10R in $\mathrm{CD} 16^{+}$monocytes. As such, the possible mechanism of GA on interacting subsets could be a downregulation of IL-10R in CD $16^{+}$monocytes which prevents the negative feedback from high production levels of IL-10. As a result, instead of affecting the $\mathrm{CD}_{16}{ }^{+}$monocytes, the GA-induced IL-10 cytokine may preferentially inhibit the inflammatory phenotype of $\mathrm{CD} 14^{+}$monocytes and thus, enhance the type II activation of this subset by GA-treated $\mathrm{CD} 16^{+}$monocytes. All together these findings suggest that modulation of IL-10R may possibly be another pathway by which GA affects monocyte subsets and their interactions through paracrine mechanisms.

\subsection{CONCLUSIONS}

1. This study demonstrated that GA and IC treatments had a direct type II activating effect on individual $\mathrm{CD} 14^{+}$and $\mathrm{CD} 16^{+}$monocyte subsets, isolated from MS patients.

2. The GA and IC combination treatment is more effective in the type II activation of the monocyte subsets.

3. Although an interaction between $\mathrm{CD} 14^{+}$and $\mathrm{CD} 16^{+}$monocytes negatively affected their response to IC treatment, it improved the type II activating effect by GA. The impaired IC efficacy could be ameliorated by a combination of IC with GA, in MS patients.

4. This study shows, for the first time, unique characteristics of $\mathrm{CD} 14^{+}$and $\mathrm{CD} 16^{+}$ monocyte subsets and their response to IC, GA and combination treatments in a healthy New Zealand population and New Zealand MS patients. 
Chapter 6.

Type II activation of monocytes by GA in vivo 


\subsection{INTRODUCTION}

While the in vitro studies showed a direct type II activating effect of GA on human monocytes (Chapters 4 and 5), it is not clear whether GA has a type II activating effect on monocytes in vivo; where the monocytes encounter a more complex immune environment. In vivo GA treatment affects a diverse repertoire of immune cells, including Th1[21], Th17 and dendritic cells, as well as monocytes, altering them to a less inflammatory phenotype[218]. Although the main GA effect on monocytes is thought to be mediated by T cells[21], our study showed that GA can directly affect monocytes in vitro (Chapter 4). In addition, it has previously been shown that inhibition of inflammatory cytokine production by both Th1 and Th17 cells does not correlate with a relapse risk in MS patients[218], indicating that modulation of $\mathrm{T}$ cells may not be the main mechanism of GA action on the disease course. Furthermore, GA's effect on cells of monocytic lineage appears to be more important in the modulation of the immune system during MS. This has been shown by Sellebjerg et al. who found that GA downregulates expression of CD40 in a differentiated form of monocytes, such as dendritic cells, a high level of which correlates with a relapse risk in MS[218]. Moreover, human monocytes have a lower expression of inflammatory markers CD86[218], SLAM and TNF $\alpha[32]$ in GA-treated patients in comparison to non-GA treated patients, and GA-treated monocytes reverse the disease progression in mice model of MS[43]. Therefore, modulation of monocytes by GA, shown in our in vitro studies (Chapters 4 and 5), is an essential part of GA action on immune system in vivo.

Whilst these studies show the importance of monocyte involvement in mediation of GA's effect on the immune system, the in vivo type II activating effect of GA on monocytes has not yet been described. Hence, this chapter aims to characterize the type II activating effect of GA on monocytes from MS patients in vivo. Additionally, this study also investigates whether in vivo GA-treated monocytes can be further modulated by additional in vitro GA and IC treatments. 


\subsection{AIMS}

This study aims to investigate the type II activating effect of GA on monocytes in MS patients in vivo. This broad aim will be addressed by the following specific aims:

1. To characterize in vivo GA effect on monocyte phenotype in MS patients.

2. To explore in vitro classical activation of monocytes from in vivo GA-treated MS patients in comparison to non-GA treated MS patients.

3. To characterize in vitro type II activation of monocytes from in vivo GA-treated MS patients by GA and IC and compare these to non-GA treated MS patients. 


\subsection{RESULTS}

\subsubsection{Proportion of monocyte subsets from in vivo GA-treated MS patients}

This study has previously shown that although the proportion of total monocyte population in the total PBMC is within the normal range, the balance between the $\mathrm{CD} 14^{+}$and $\mathrm{CD} 16^{+}$monocytes is significantly altered in MS patients, with an increase in the proportion of $\mathrm{CD}_{16}{ }^{+}$monocytes (Chapter 3). In patients treated with GA the imbalance in the proportion of subsets was even greater than in healthy and non-GA treated MS groups. As a consequence, the proportion of $\mathrm{CD} 14^{+}$monocytes in the in vivo GA-treated patients was significantly lower than in the healthy and non-GA treated MS groups (Table 6.1). Thus, in comparison to the non-GA treated MS group, in which a five-fold increase was observed in the proportion of $\mathrm{CD} 16^{+}$monocytes, the in vivo GA-treated group showed an eight-fold increase. This increase in the proportion of $\mathrm{CD} 16^{+}$monocytes resulted in an almost equal proportion of $\mathrm{CD}^{4} 4^{+}$and $\mathrm{CD}_{1} 6^{+}$monocytes in the GA-treated patients (Figure 6.1.c); whereas, the $\mathrm{CD}_{1} 4^{+}$subset constituted the majority of monocytes in healthy and non GAtreated MS groups (Figures 6.1.a,b). 


\begin{tabular}{|l|rl|rl|}
\hline Subject groups & \multicolumn{2}{|c|}{ CD14 ${ }^{+}$monocytes } & \multicolumn{2}{|c|}{ CD16 $^{+}$monocytes } \\
\hline Healthy & $92 \% \quad \pm 4$ & $5,1 \%$ & \pm 3 \\
\hline Non-GA treated MS & $73,6 \%$ & \pm 17 & $26,2 \%$ & \pm 17 \\
\hline GA-treated MS & $56,4 \%$ & \pm 13 & $42,9 \%$ & \pm 13 \\
\hline
\end{tabular}

Table 6.1. Proportion of CD14 ${ }^{+}$and $\mathrm{CD}_{16}^{+}$monocytes.

Total monocytes from healthy subjects $(n=23)$, non-GA treated MS patients $(n=20)$ and GA-treated MS patients $(n=7)$ were isolated from PBMC using CD14 Microbeads and stained with anti-CD14 and CD16 antibodies. The subset proportion was assessed in total monocytes based on the expression of CD14 and CD16 by flow cytometry. MS patients received daily $20 \mathrm{mg}$ subcutaneous injection of GA for minimum of 6 months. Shown are the means and SD of individuals in healthy, non-GA treated MS, and GA-treated MS groups. Data were collected on flow cytometry using the gating strategy in appendix 8 .
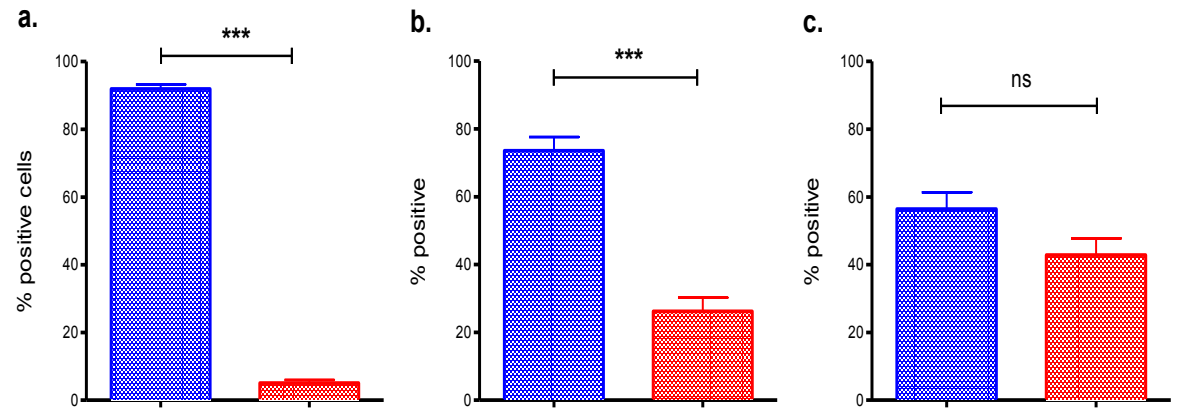

CD14+ monocytes

$\mathrm{B}$ CD16+ monocytes

\section{Figure 6.1. Proportion of $\mathrm{CD}^{+} 4^{+}$and $\mathrm{CD}^{+}{ }^{+}$monocytes was altered in MS patients.}

Total monocytes from healthy subjects $(n=23)$, non-GA-treated MS patients $(n=20)$ and GA-treated MS patients $(\mathrm{n}=7)$ were isolated from PBMC using CD14 Microbeads and stained with anti-CD14 and CD16 antibodies. The subset proportion was assessed in total monocytes based on the expression of CD14 and CD16 by flow cytometry. (a) Subset proportion in healthy subjects. (b) Subset proportion in non-GA-treated MS patients. (c) Subset proportion in GA-treated MS patients. MS patients received daily $20 \mathrm{mg}$ subcutaneous injection of GA for minimum of 6 months. Shown are the means and SD of individuals in healthy, non-GA-treated MS, and GAtreated MS groups. $* * * p<0.001$ by Student's t-test. Data were collected on flow cytometry using the gating strategy in appendix 8. 


\subsubsection{Type II activation of monocytes and their subsets by in vivo GA treatment}

Activation marker expression on $\mathrm{CD}_{1} 4^{+}$and $\mathrm{CD}_{16}{ }^{+}$subsets were examined in freshly isolated monocytes from MS patients, who had received daily GA injections for at least 6 months. Overall, monocytes and their subsets from in vivo GA-treated patients had characteristics of type II activated cells. In particular, the expression of CD86 and CD64 was significantly lower in GA-treated MS patients, compared to non-GA-treated MS patients while CD40 and HLA-DR also showed a modest reduction. However, the expression of CCR2 was slightly increased, in comparison to the non-GA-treated patients (Figure 6.2). This suggests that in addition to the type II activating effect, in vivo GA treatment maintains an ability of monocytes to migrate in the response to CCR2 ligand, CCL2.
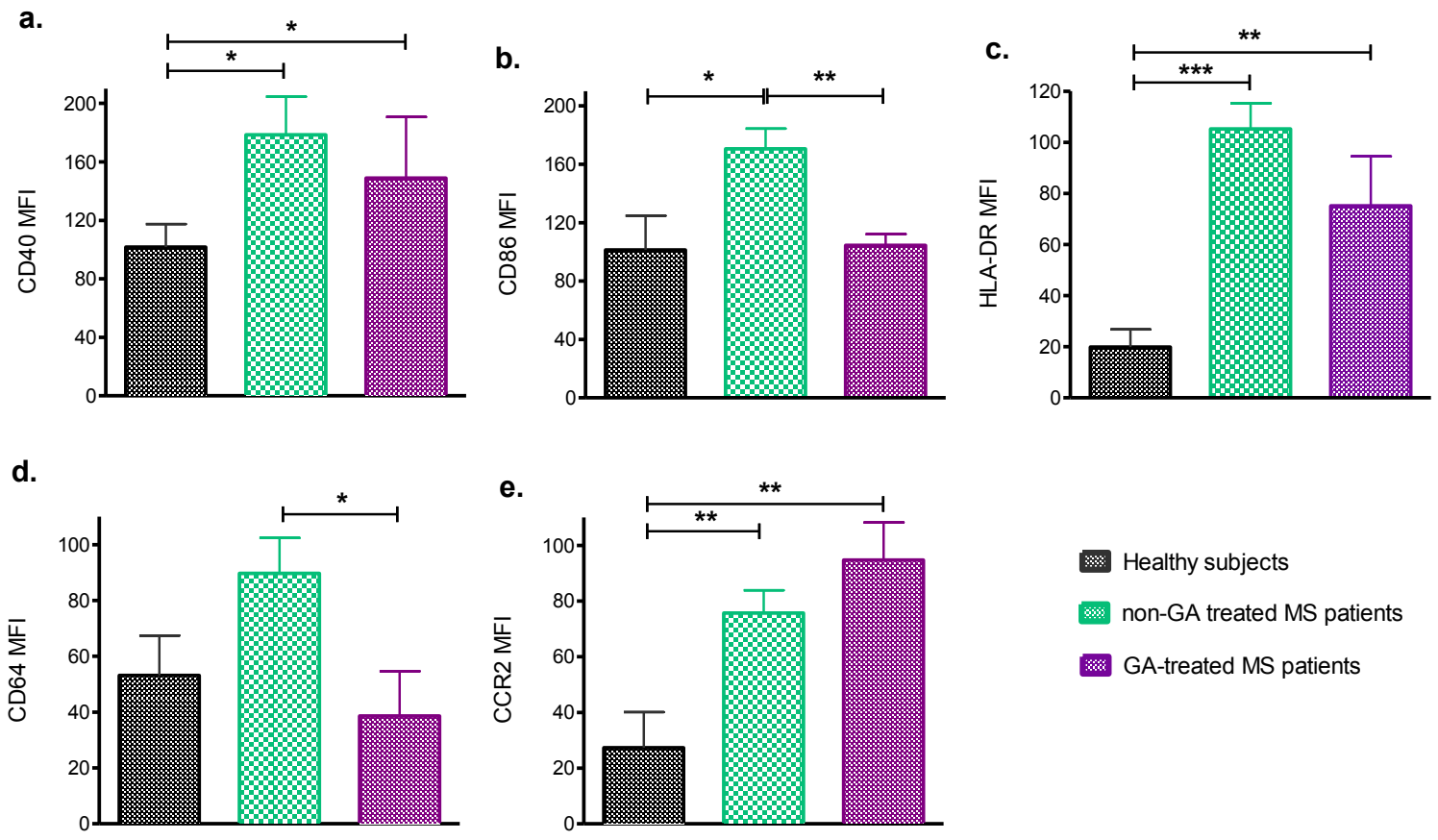

Figure 6.2. Type II activation of total monocytes by the in vivo GA treatment of MS patients.

Total monocytes from healthy subjects $(n=23)$, non-GA-treated MS patients $(n=20)$ and GA-treated MS patients $(\mathrm{n}=7)$ were isolated from PBMC using CD14 Microbeads and stained with antibodies. (a-e) Marker expression was assessed by flow cytometry for (a) CD40, (b) CD86, (c) HLA-DR, (d) CD64, and (e) CCR2 expression (MFI). MS patients in GA-treated group received daily $20 \mathrm{mg}$ subcutaneous injection of GA for minimum of 6 months. Shown are the means and SD of individuals from the three groups. ${ }^{*} \mathrm{p}<0.05 ; * * \mathrm{p}<0.01 ;{ }^{*} * \mathrm{p}<0.001$ by one way ANOVA with Dunnett's post test. Data were collected on flow cytometry using the gating strategy in appendix 9. 
In order to determine, if GA differentially affected the two monocyte subsets, the expression of activation markers was determined on ex vivo isolated CD14 ${ }^{+}$and $\mathrm{CD} 16^{+}$ monocyte subsets. Both $\mathrm{CD} 14^{+}$and $\mathrm{CD}_{1} 6^{+}$subsets from GA-treated patients had a lower expression of CD86, HLA-DR and CD64, whereas expression of CCR2 was slightly increased compared to non-GA-treated MS patients. Unlike CD14 $4^{+}$monocytes, which did not show any alteration in CD40, CD16 $6^{+}$monocytes had downregulated expression of CD40 in GA-treated patients (Table 6.2.b). Overall, these results suggest that monocytes and their subsets from MS patients can be type II activated by the in vivo GA treatment.

\subsubsection{Comparison of in vivo and in vitro GA effect on monocytes and their subsets}

In vitro type II activation of monocytes by GA was assessed by alteration of activation marker expression in relation to in vitro classically activated monocytes, whereas an in vivo GA effect was examined in monocytes from GA-treated patients in relation to non-GAtreated patients. When the in vivo GA effect was compared to the in vitro GA effect, the in vivo GA-treated monocytes of both subsets had greater type II activation. While the in vitro GA treatment inhibited the expression of CD40 and CD86 in total monocyte population and CD40 in $\mathrm{CD} 16^{+}$monocytes only, the in vivo GA-treated monocytes had decreased expression of CD40, CD86, HLA-DR and CD64 in total monocytes and subsets, except for CD40 in $\mathrm{CD}_{14}{ }^{+}$monocytes. Furthermore, although in vitro GA treatment did not alter the expression of CCR2, in vivo GA treatment resulted in a modest increase in CCR2 in both subsets from MS patients (Table 6.2.a and b). This increase did not quite reach significance due to the lower number of GA-treated patients $(n=7)$. Further work therefore needs to be carried out to confirm these findings. These results indicate that GA had a strong type II activating effect on monocytes in vivo when MS patients received daily GA injections for minimum period of 6 months; whereas, during the in vitro treatment monocytes only had a single dose of GA. 
a.

\begin{tabular}{|l|c|c|c|c|c|c|}
\hline & \multicolumn{3}{|c|}{ GA effect in vitro $(\mathrm{n}=20)$} & \multicolumn{3}{c|}{ GA effect in vivo (n=7) } \\
\cline { 2 - 7 } & Total mo & $\mathbf{C D 1 4}^{+} \mathbf{m o}$ & $\mathbf{C D 1 6}^{+}$mo & Total mo $^{*} \mathbf{C D 1 4}^{+}$mo & CD16 $^{+}$mo \\
\hline CD40 & $\downarrow^{*}$ & & $\downarrow^{*}$ & $\downarrow$ & & $\downarrow$ \\
\hline CD86 & $\downarrow^{*}$ & & & $\downarrow^{*}$ & $\downarrow^{*}$ & $\downarrow^{*}$ \\
\hline HLA-DR & & & & $\downarrow$ & $\downarrow$ & $\downarrow^{*}$ \\
\hline CD64 & $\downarrow$ & $\downarrow$ & $\downarrow$ & $\downarrow^{*}$ & $\downarrow^{*}$ & $\downarrow^{*}$ \\
\hline CCR2 & & & & $\uparrow$ & $\uparrow$ & $\uparrow$ \\
\hline
\end{tabular}

Table 6.2. Comparison of in vivo and in vitro GA effects on monocytes and their subsets in MS patients.

(a)Total monocytes and monocyte subsets from non-GA treated MS patients $(n=20)$ were isolated from PBMC, primed and treated with $100 \mu \mathrm{g} / \mathrm{ml} \mathrm{GA}$ in the presence of LPS for 24 hours. The monocytes were then harvested, stained with flow antibodies and assessed for marker expressions by flow cytometry. Data are shown in comparison to the LPS treatment. (b) MS patients in GA-treated group $(\mathrm{n}=7)$ received daily $20 \mathrm{mg}$ subcutaneous injections of GA for minimum of 6 months. The total monocytes and monocyte subsets were isolated, stained with flow antibodies and assessed for marker expressions by flow cytometry. Data are shown in comparison to the non-GA treated MS group. Shown are the means and SD from individual experiments. The statistical analysing was done by using one way ANOVA with Dunnett's post test to compare three or more groups and Student's t-test to compare 2 groups. Dark green box shows significant decrease; light green - nonsignificant decrease; pink - non-significant increase; white - no change in the expression. 


\subsubsection{Classical activation of in vivo GA-treated monocytes}

Previous work has shown that classical activation of healthy monocytes exposed to GA in vitro leads to an activation phenotype similar to type II activation; however, whether these changes can occur after in vivo GA treatment are unknown. To assess how in vivo GA treatment affected the ability of monocytes to become classically activated, monocytes from GA-treated MS patients were isolated, primed overnight with IFN- $\gamma$ and activated with LPS for 24 hours. Although the expression of CCR2 was increased in the total monocyte population, the production of inflammatory cytokines IL-6 and IL-12 are significantly lower than in non-GA-treated MS patients. Additionally, monocytes from GA-treated individuals had reduced expression of CD40, CD86 and HLA-DR, with levels similar to those found in healthy subjects (Figure 6.3). The expression of these activation markers was also decreased on both $\mathrm{CD}_{1} 4^{+}$and $\mathrm{CD} 16^{+}$subsets suggesting that in vivo GA treatment reduces the ability of monocytes and their subsets to become classically activated upon in vitro LPS stimulation, and this process is different from monocytes from non-GA-treated MS patients. 
a.

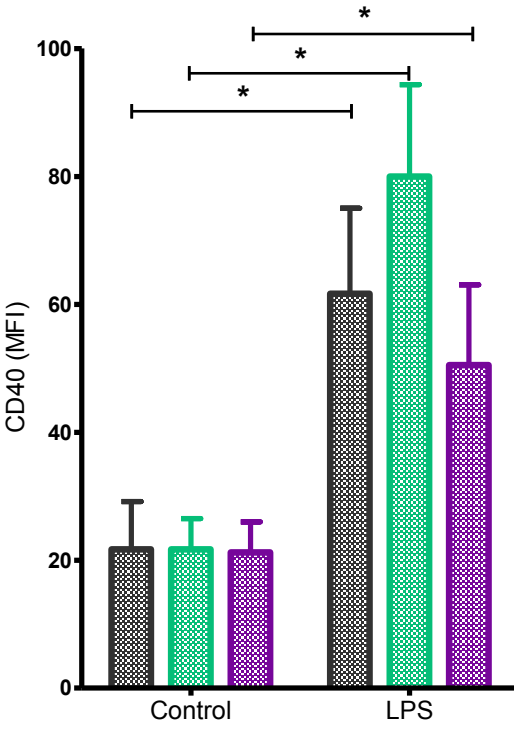

d.

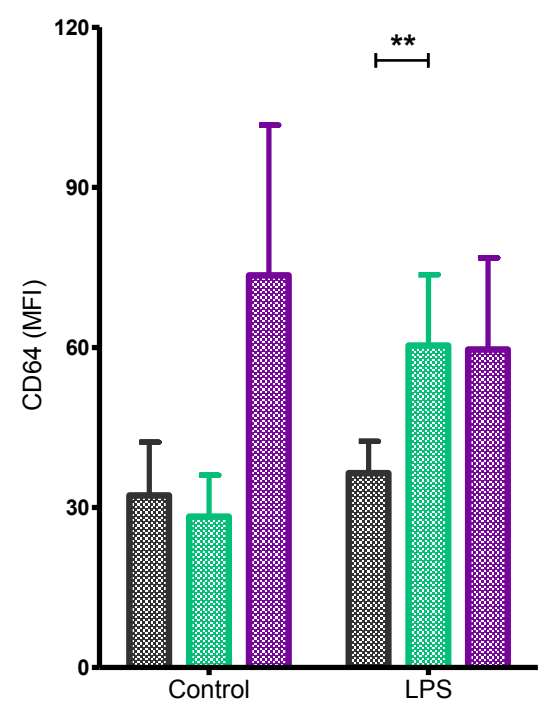

g.

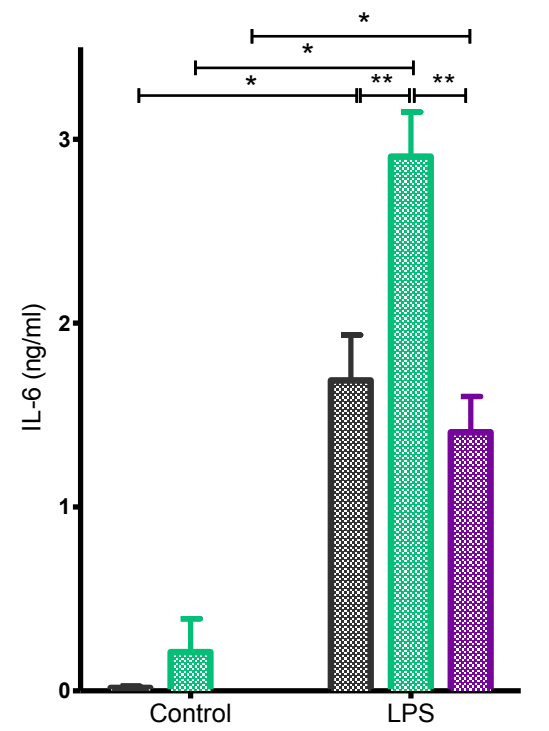

b.

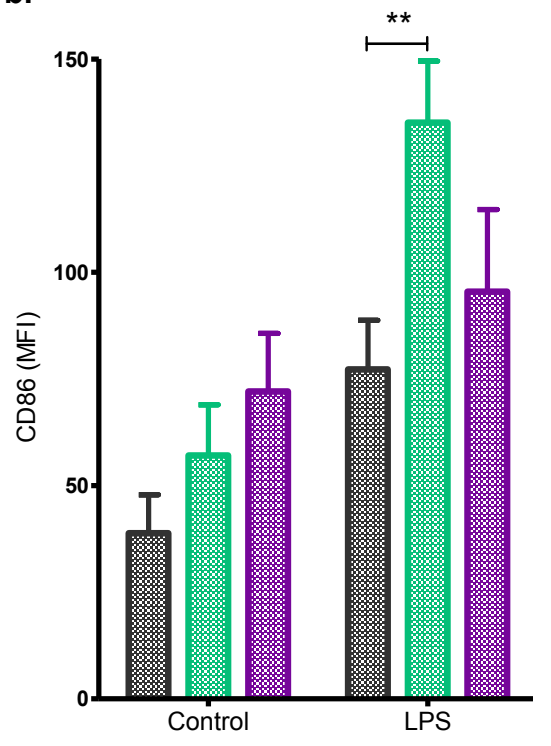

e.

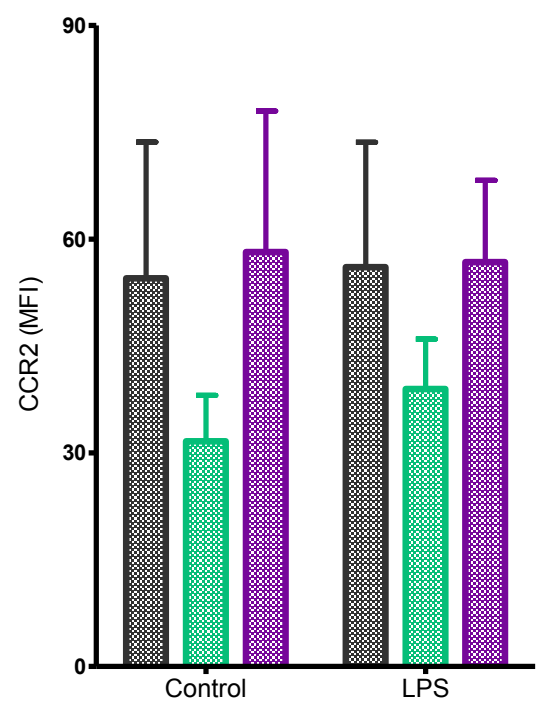

h.

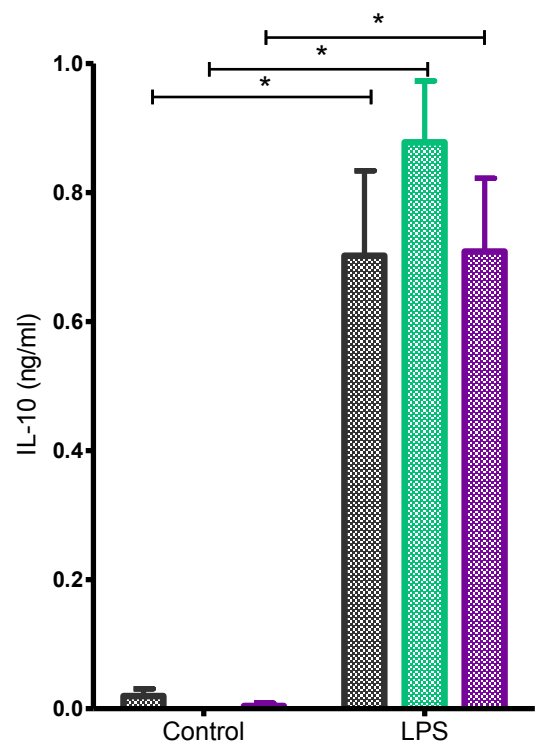

c.

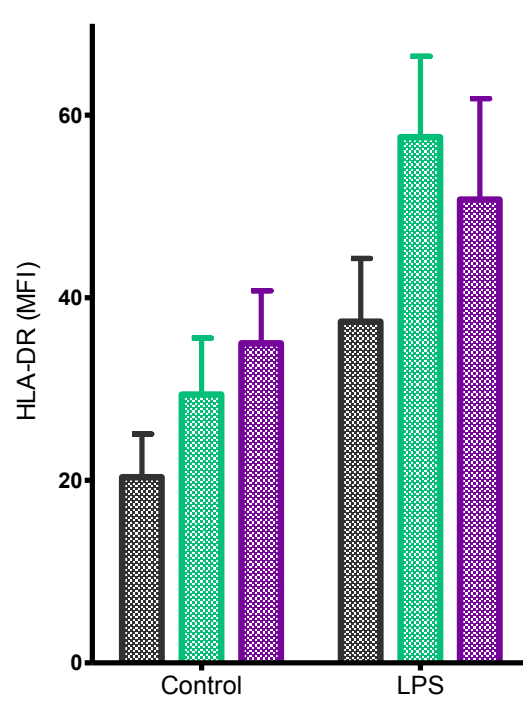

f.

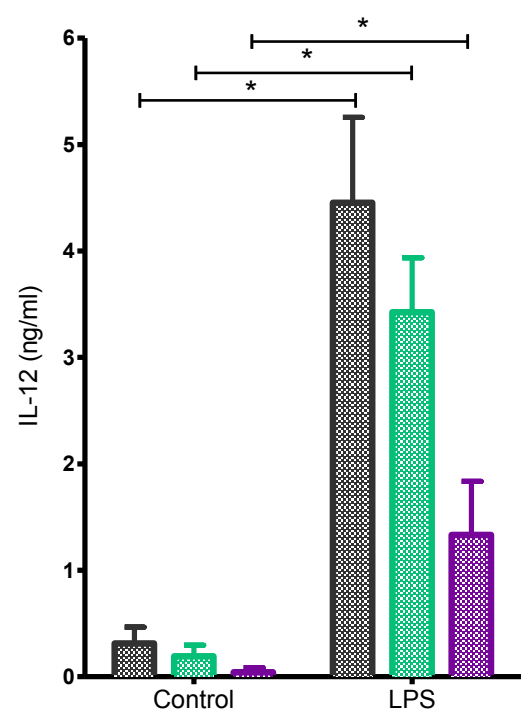

Healthy subjects

non-GA treated MS patients

\% GA-treated MS patients 
Figure 6.3. Monocytes from GA-treated MS patients had reduced ability for classical activation in vitro.

MS patients in GA-treated group ( $\mathrm{n}=7$ ) received daily $20 \mathrm{mg}$ subcutaneous injections of GA for minimum of 6 months. Total monocytes were isolated from healthy subjects $(n=23)$, non-GA-treated MS patients $(n=20)$ and GA-treated MS patients. The monocytes were primed with IFN- $\gamma$ and stimulated with LPS for 24 hours and stained with antibodies. The activation marker expression was assessed by flow cytometry, and cytokine production was measured by ELISA. The control group represents unstimulated and untreated monocytes. (a) Expression of CD40 on monocytes. (b) Expression of CD86 on monocytes. (c) Expression of HLA-DR on monocytes. (d) Expression of CD64 on monocytes. (e) Expression of CCR2 on monocytes. (f) Production of IL12 in monocytes. (g) Production of IL-6 in monocytes. (h) Production of IL-10 in monocytes. Shown are the means and SD from individuals from the three groups. ${ }^{*} \mathrm{p}<0.01 ; * * \mathrm{p}<0.005 ; * * * \mathrm{p}<0.001$ by one way ANOVA with Dunnett's post-test. Data were collected on flow cytometry using the gating strategy in appendix 9. 


\subsubsection{In vitro GA effect on in vivo GA-treated monocytes and their subsets}

To determine if in vitro GA treatment of monocytes from GA-treated MS patients had an additional effect, monocytes from healthy subjects, non-GA-treated and GA-treated MS patients were cultured with LPS and GA. However, this additional exposure to GA did not alter the expression of the monocyte activation markers significantly and induced only a very slight decrease in the expression of CD40, CD86, HLA-DR and CD64. In contrast, the production of IL-6 and IL-12 were significantly lower than in non-GA-treated patients (Figure 6.4), suggesting that the in vivo GA-exposed monocytes still retained responsiveness to GA in vitro.

\subsubsection{In vitro IC effect on in vivo GA-treated monocytes and their subsets}

To assess if in vivo GA treatment altered the ability of monocytes to be type II activated by IC, monocytes from GA-treated MS patients were type II activated by IC and compared to monocytes from healthy subjects and non-GA-treated MS patients. Similar to in vitro GA treatment, the additional in vitro IC treatment of the in vivo GA-exposed monocytes and their subsets did not alter the expression of the activation markers CD40, CD86, HLA-DR, and CCR2 on monocytes or on their subsets, nor was the production of IL-12 altered. However, the production of IL-6 was significantly inhibited by IC in monocytes from GA-treated MS patients in comparison to the non-GA-treated MS patients (Figure 6.4). Furthermore, the combined in vitro IC and GA treatment significantly enhanced the inhibitory effect of the in vivo GA treatment on the production of IL-6, IL-12 and CD40 in the total monocyte population in MS patients (Figure 6.4). Thus, while monocytes exposed to GA in vivo were far less responsive to subsequent in vitro GA or IC treatment alone, the combined exposure of monocytes to IC and GA led to the greatest level of type II activation. 
a.

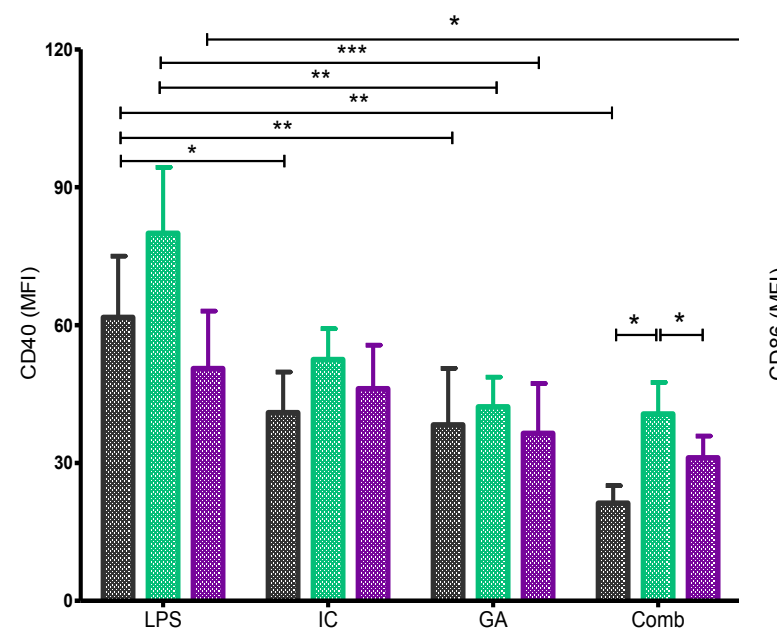

b.

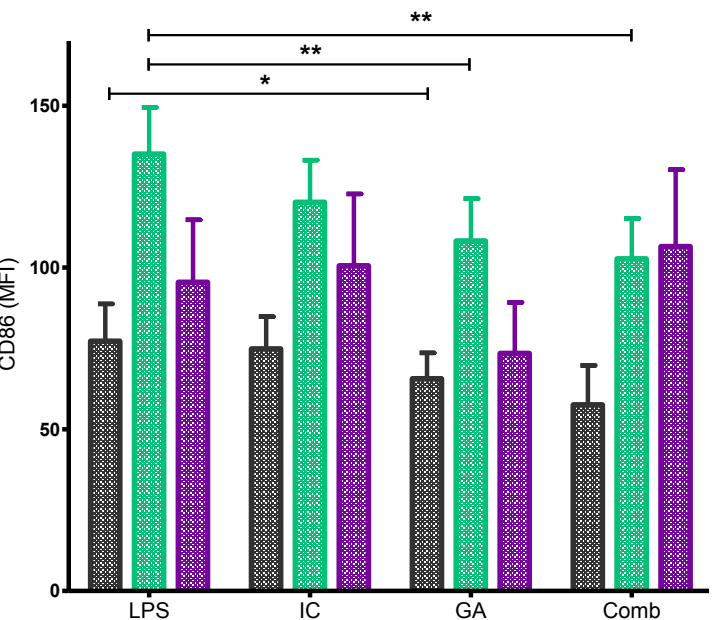

d.

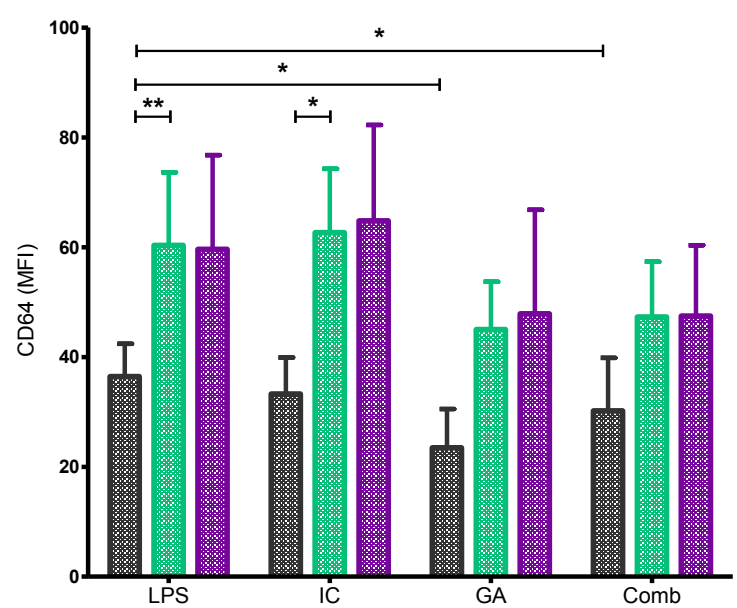

f.

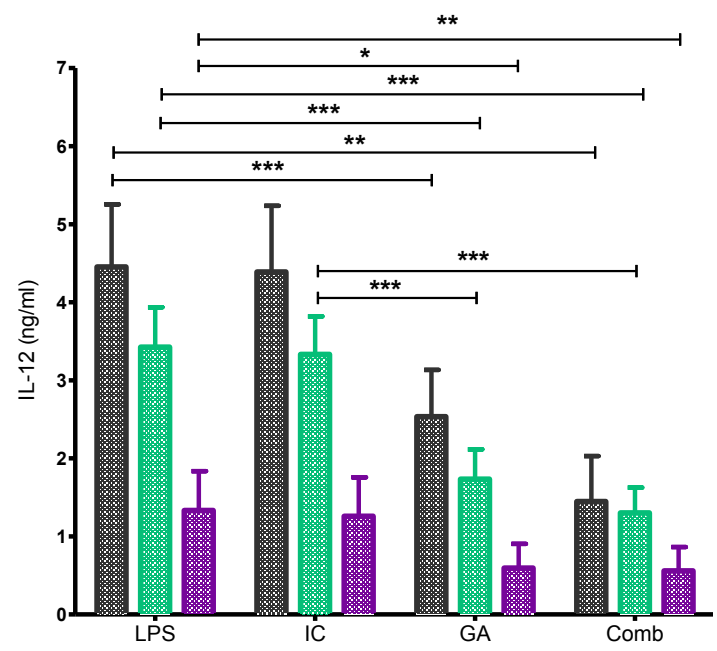


g.

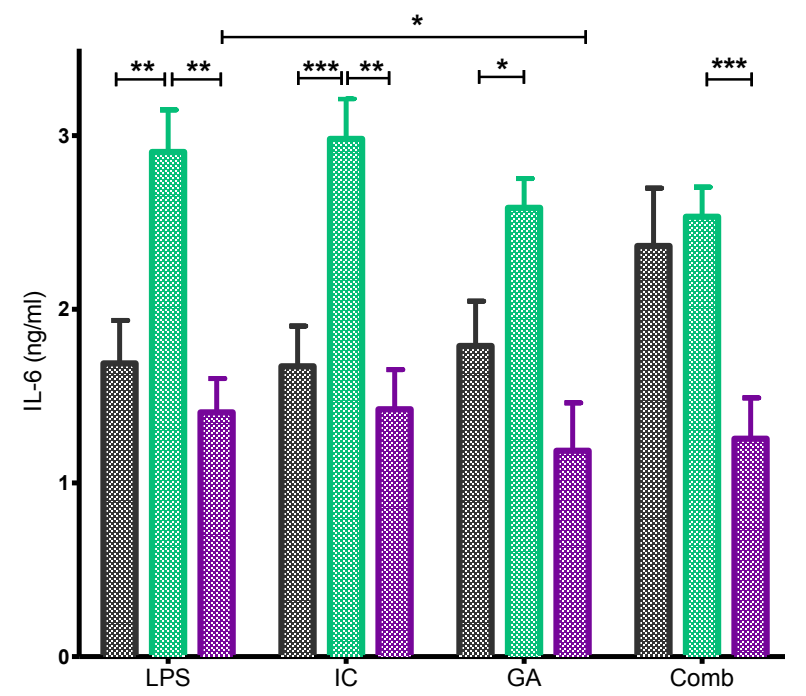

h.

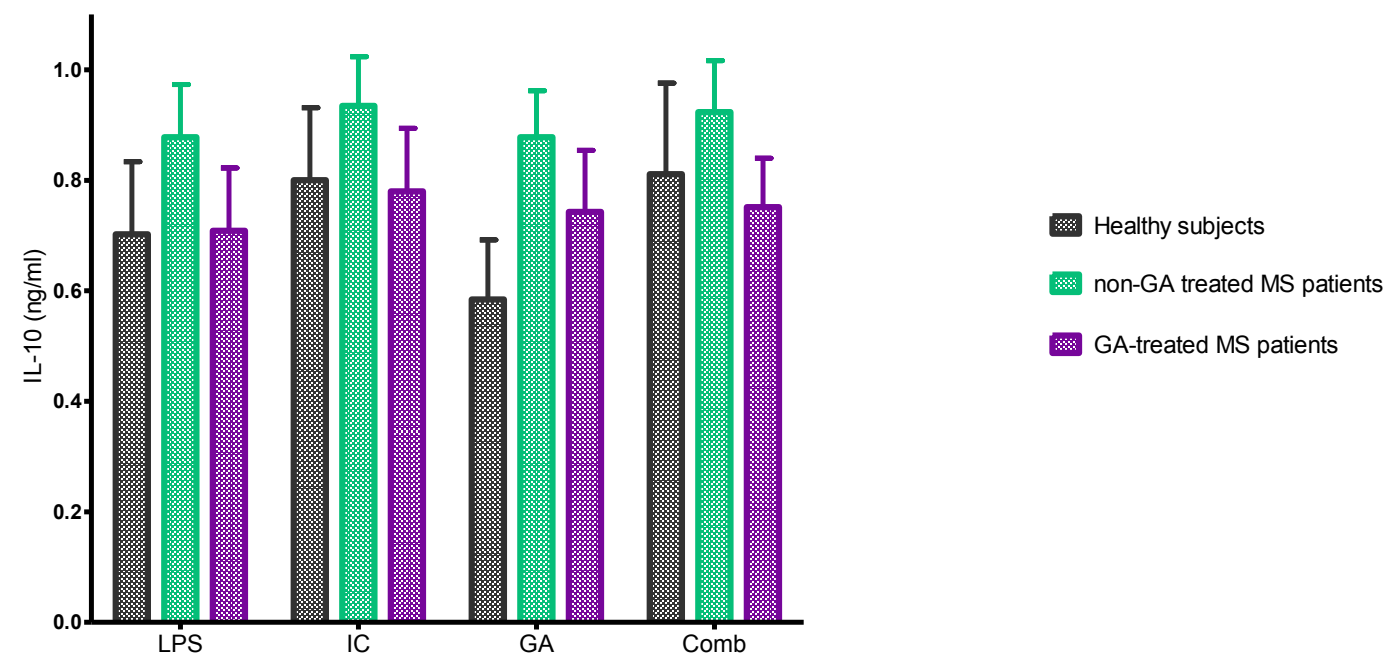

Figure 6.4. In vitro GA and IC treatment enhances the in vivo type II activating effect of GA.

MS patients in GA-treated group $(\mathrm{n}=7)$ received daily $20 \mathrm{mg}$ subcutaneous injections of GA for minimum of 6 months. Total monocytes were isolated from healthy subjects $(n=23)$, non-GA treated MS patients $(n=20)$ and GA-treated MS patients. The monocytes were primed with IFN- $\gamma$, treated with GA and/or IC in the presence of LPS for 24 hours and stained with antibodies. The activation marker expression was assessed by flow cytometry, and cytokine production was measured by ELISA. (a) Expression of CD40 on monocytes. (b) Expression of CD86 on monocytes. (c) Expression of HLA-DR on monocytes. (d) Expression of CD64 on monocytes. (e) Expression of CCR2 on monocytes. (f) Production of IL-12 in monocytes. (g) Production of IL-6 in monocytes. (h) Production of IL-10 in monocytes. Shown are the means and SD from individuals from the three groups. ${ }^{*} \mathrm{p}<0.05 ; * * \mathrm{p}<0.01 ; * * * \mathrm{p}<0.001$ by one way ANOVA with Dunnett's post-test. Data were collected on flow cytometry using the gating strategy in appendix 9. 


\subsubsection{Difference in monocyte activation between the subject groups}

While the individual analysis of cytokines and surface markers is important in understanding the molecular changes, induced by disease or by various treatments, this analysis does not consider the pattern of expression in an individual. To compare the pattern of activation of monocytes from individuals from different groups (i.e. healthy, non-GAtreated MS and GA-treated MS groups), a hierarchical cluster analysis (HCA) of the ex vivo monocyte phenotype was performed. HCA is an unsupervised multivariate analysis that clusters groups, based on multiple variables (in this case different cell surface markers or $\%$ of cells), and shows the similarities and differences by a dendrogram and heat map. The HCA analysis revealed that all but three of the subjects (h-24, MS-20 and GA-2) formed three distinct groups that aligned with their disease state. This was dependent on the expression of activation markers CD40, CD86, HLA-DR and CD64 on total monocyte population and on the subsets, as well as proportion of their $\mathrm{CD} 14^{+}$and $\mathrm{CD} 16^{+}$subsets (Figure 6.5). The first group (Figure 6.5.a) included the healthy subjects and the second group non-GA treated MS patients (Figure 6.5.b). The third and last group consisted of in vivo GA-treated MS patients (Figure 6.5.c).

According to the dendrogram, monocytes from healthy subjects and GA-treated MS patients had a similar low expression of activation markers, indicative of a reduced activation state in these two groups (Figures 6.5.a and c). However, a difference in the proportion of $\mathrm{CD}_{16}{ }^{+}$monocytes was evident between these two groups, where the healthy group had a lower number of $\mathrm{CD}_{1} 6^{+}$monocytes and the GA-treated MS group had the highest number of $\mathrm{CD}_{16}{ }^{+}$monocytes out of all three groups. Unlike healthy and GA-treated MS groups, a very distinct pattern was observed in the non-GA treated MS group, which had a high expression of activation markers, suggesting a high activation state of the monocytes (classical activation) in this group (Figure 6.5.b).

The exceptions to the clustering are the subjects h-24, MS-20 and GA-2, and these exceptions can be due to several possible issues. Firstly the healthy subject h-24 had a pattern similar to the MS group, suggesting that these monocytes were more activated than monocytes from other healthy individuals possibly due to a subclinical infection. The subject MS-20 from the non-GA-treated MS group stopped GA treatment 7 months prior to this study, and thus residual GA-mediated effects may still be present. Finally, the subject GA-2 from GA-treated MS group had shown resistance to GA treatment, such that GA was having 
no effect on the disease progression. Despite these exceptions, all other subjects have been uniformly grouped during the analysis supporting the ability of this analysis to distinguish these groups. Furthermore, while monocytes from healthy and GA-treated MS groups had similar characteristics, the monocytes from the non-GA-treated MS group had a very distinct pattern unlike the other groups. In summary, the three subject populations, i.e. healthy, nonGA-treated MS and GA-treated MS groups, had unique characteristics based solely upon their monocyte activation phenotype and subset proportion, which allowed them to cluster into three distinct groups.

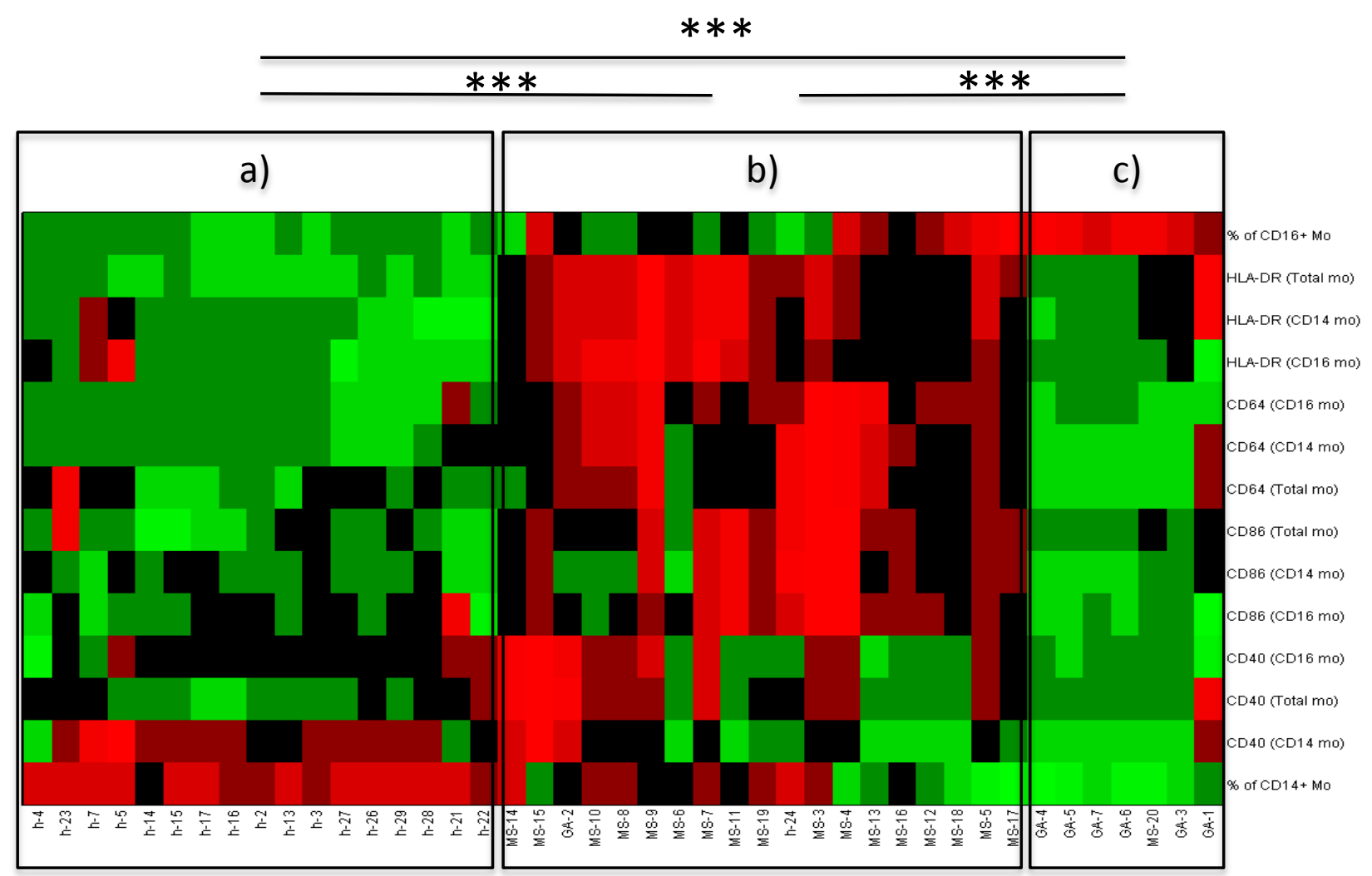

Figure 6.5. Hierarchical cluster analysis dendrogram and heat map of the three subject groups based on their ex vivo characteristics.

Total monocytes and monocyte subsets were isolated from healthy subjects ( $n=23)$, non-GA-treated MS patients $(n=20)$ and GA-treated MS patients groups $(n=7)$. The activation marker expression was assessed by flow cytometry. The MFI values for activation markers and the proportion of monocyte subsets as a percentage of total monocyte population have been analyzed in this study. Green shows low expression, black - medium expression, red - high expression. The lowest expression is shown as dark green and the highest as dark red. Data were collected on flow cytometry using the gating strategy in appendix 8 . 


\subsubsection{Comparison of monocyte activation between the subject groups after in vitro treatments}

In order to compare the pattern of activation of monocytes treated with LPS, GA and IC in vitro, isolated monocytes from the three groups (non-GA treated MS, GA-treated MS and healthy groups) were primed with IFN- $\gamma$ and stimulated with LPS in the presence or absence of GA and IC. HCA analysis of in vitro LPS-stimulated monocytes demonstrated two distinct patterns of monocyte activation, depending on marker and cytokine levels in monocytes. Pattern 1 included subjects with a low level of classical activation of monocytes and pattern 2 included subjects with a high activation (Figure 6.6). Interestingly, the majority of GAtreated MS patients $(71 \%)$ and healthy subjects $(64 \%)$ were the pattern 1 , where the majority of non-GA treated MS patients (80\%) were in the pattern 2 (Table 6.3). As seen with the ex vivo analysis, healthy subject h-24 and GA-treated MS patient GA-2 were in the group of non-GA treated MS patients. These results suggest that most healthy subjects and GA-treated MS patients have a similar low level of classical activation of monocytes, which allows them to fall into one group. In contrast, non-GA treated MS patients form a distinct group with a higher ability of monocytes to undergo a classical activation, in comparison to the other groups. 


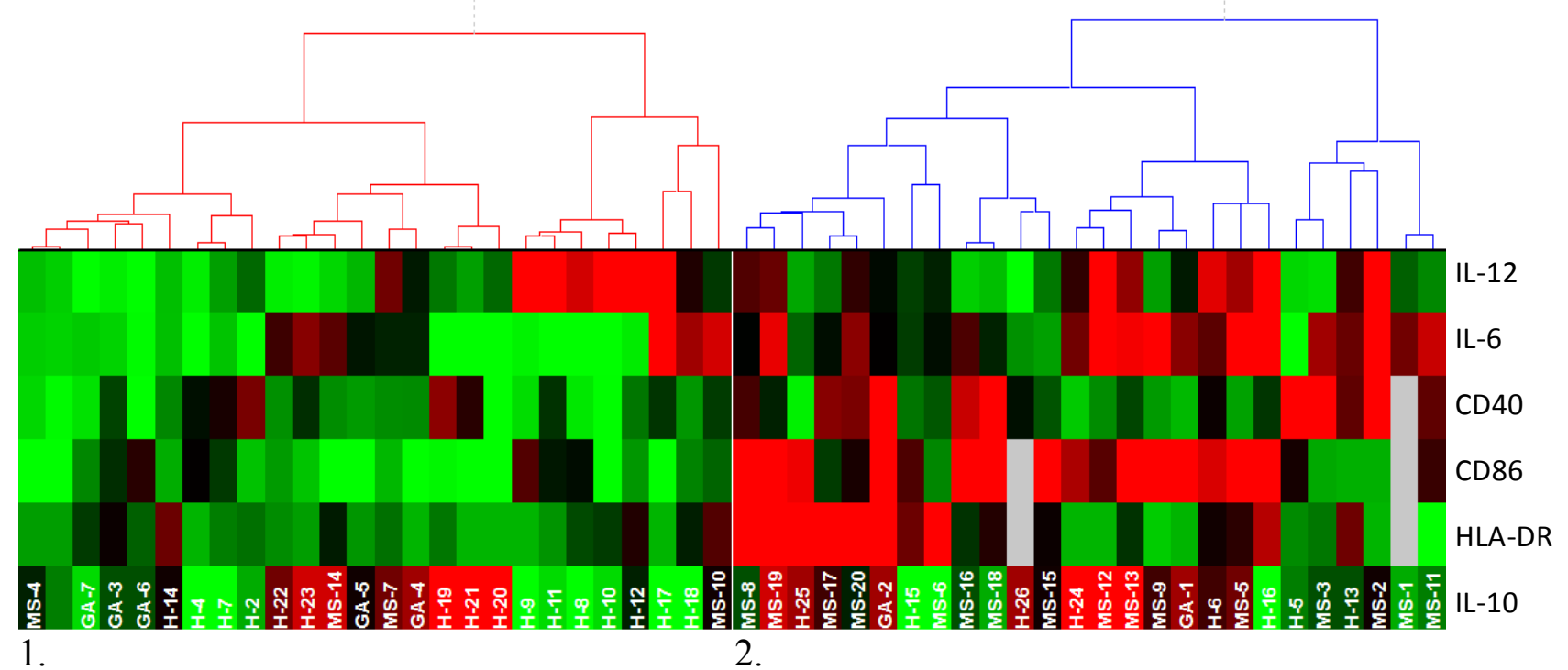

Figure 6.6. HCA dendrogram and heat map of in vitro LPS-stimulated total monocytes.

Total monocytes were isolated from healthy subjects $(n=24)$, non-GA-treated MS patients $(n=20)$ and GAtreated MS patients $(n=7)$. The monocytes were primed with IFN- $\gamma$ and stimulated with LPS for 24 hours. The activation marker expression was assessed by flow cytometry, and cytokine production was measured by ELISA. The MFI values of activation markers and concentrations of cytokines have been analyzed in this study. (1) Pattern 1. (2) Pattern 2. Green shows low expression, black - medium expression, red - high expression. The lowest expression is shown in dark green and the highest in dark red. Dendrogram represents the same data as in the figure 6.3. Data were collected on flow cytometry using the gating strategy in appendix 9.

\begin{tabular}{|l|l|l|l|}
\hline Subject groups: & Pattern 1 & Pattern 2 & Total \\
\hline Non-GA treated MS & $4(20 \%)$ & $16(80 \%)$ & $\mathbf{2 0}(\mathbf{1 0 0} \%)$ \\
\hline GA-treated MS & $5(71 \%)$ & $2(29 \%)$ & $\mathbf{7 ( 1 0 0 \% )}$ \\
\hline Healthy & $16(67 \%)$ & $8(33 \%)$ & $\mathbf{2 4 ( 1 0 0 \% )}$ \\
\hline
\end{tabular}

Table 6.3. HCA of in vitro LPS-stimulated total monocytes.

Total monocytes were isolated from healthy subjects $(n=24)$, non-GA-treated MS patients $(n=20)$ and GAtreated MS patients $(n=7)$. The monocytes were primed with IFN- $\gamma$ and stimulated with LPS for 24 hours. The activation marker expression was assessed by flow cytometry and the cytokine production was measured by ELISA. Shown are the percentages of subjects (\% from the group) the clustered with the two major patterns. Data were collected on flow cytometry using the gating strategy in appendix 9. 
In vitro GA-treated monocytes formed seven different patterns of type II activation, which had a mixture of subjects from all groups (Figure 6.7). In general, in vitro GA treatment resulted in high diversity of the phenotype of type II activated monocytes between the subject groups and thus resulted in division of the subjects into small or more mixed groups. This diversity may be due to high individual variation of the ability of monocytes to respond to the GA treatment. Although a mix of groups was found in most patterns, other patterns showed a more unique grouping of subjects. Specifically, most healthy subjects and GA-treated MS subjects fell into patterns 1 and 4, with a high but different type II activation of monocytes; whereas most of the non-GA treated MS patients fell into pattern 2, in which monocytes had lowest type II activation, or pattern 6 where monocytes had the highest type II activation (Figure 6.7). This finding suggests that within a group some individuals may respond to GA better than others.

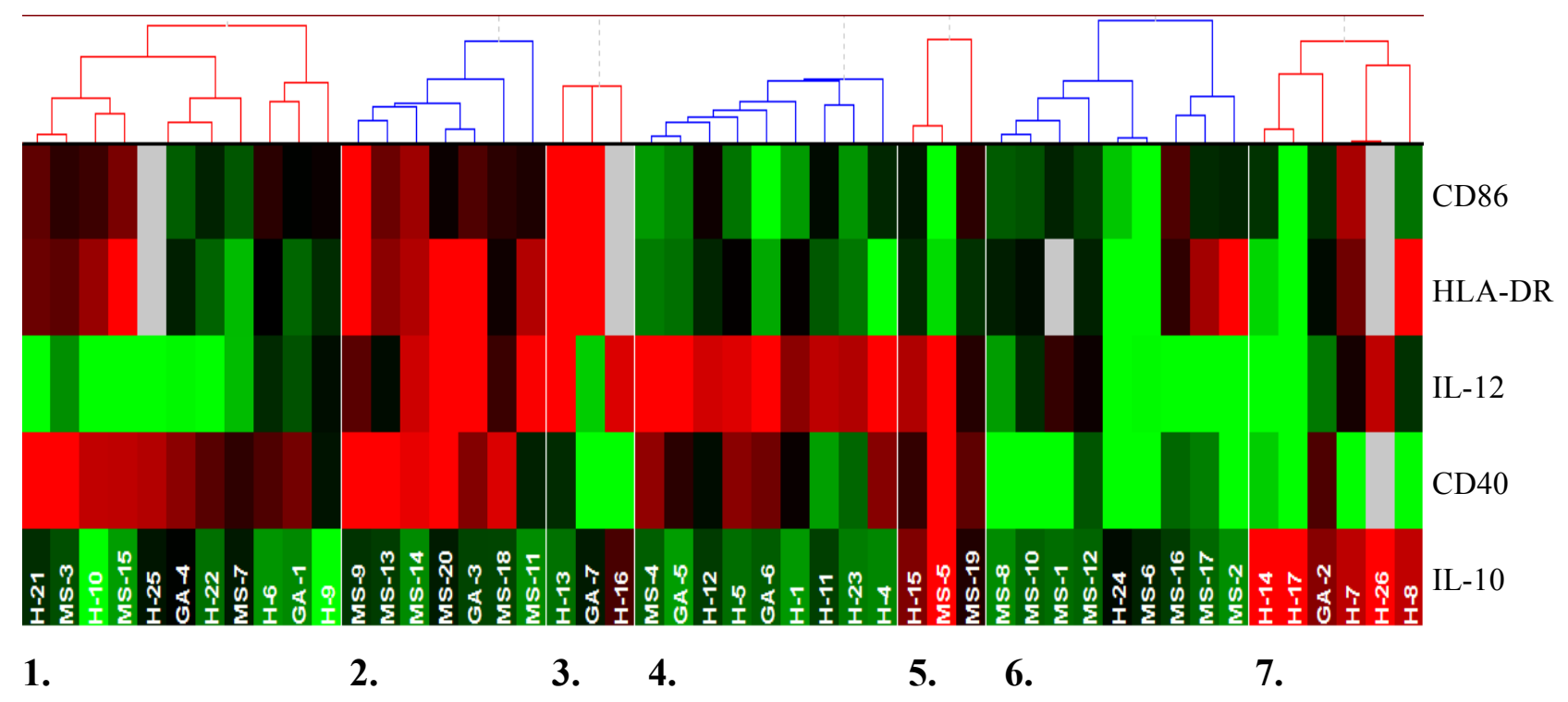

Figure 6.7. HCA dendrogram and heat map of in vitro GA-treated total monocytes.

Total monocytes were isolated from healthy subjects $(n=21)$, non-GA-treated MS patients ( $n=20)$ and GAtreated MS patients $(n=7)$. The monocytes were primed with IFN- $\gamma$ and treated with GA in the presence of LPS for 24 hours. The activation marker expression was assessed by flow cytometry, and cytokine production was measured by ELISA. The expression of activation markers and cytokines were analyzed relative to LPS stimulation alone. (1) Pattern 1. (2) Pattern 2. (3) Pattern 3. (4) Pattern 4. (5) Pattern 5. (6) Pattern 6. (7) Pattern 7. Green shows low expression, black - medium expression, red - high expression. The lowest expression is shown in dark green and the highest in dark red. Dendrogram represents the same data as in the figure 6.4. Data were collected on flow cytometry using the gating strategy in appendix 9. 


\begin{tabular}{|l|l|l|l|l|l|l|l|l|}
\hline \multicolumn{1}{|c|}{ Groups } & Pattern 1 & Pattern 2 & Pattern 3 & Pattern 4 & Pattern 5 & Pattern 6 & Pattern 7 & \multicolumn{1}{|c|}{ Total } \\
\hline $\begin{array}{l}\text { Non GA-treated MS } \\
\text { patients }\end{array}$ & $3(15 \%)$ & $6(30 \%)$ & $0(0 \%)$ & $1(5 \%)$ & $2(10 \%)$ & $8(40 \%)$ & $0(0 \%)$ & $\mathbf{2 0 ( 1 0 0 \% )}$ \\
\hline GA-treated MS & $2(29 \%)$ & $1(14 \%)$ & $1(14 \%)$ & $2(29 \%)$ & $0(0 \%)$ & $0(0 \%)$ & $1(14 \%)$ & $\mathbf{7 ( 1 0 0 \% )}$ \\
\hline Healthy & $6(29 \%)$ & $0(0 \%)$ & $2(10 \%)$ & $6(29 \%)$ & $1(5 \%)$ & $1(5 \%)$ & $5(24 \%)$ & $\mathbf{2 1 ( 1 0 0 \% )}$ \\
\hline
\end{tabular}

Table 6.4. HCA of in vitro GA-treated total monocytes.

Total monocytes were isolated from healthy subjects $(n=21)$, non-GA-treated MS patients $(n=20)$ and GAtreated MS patients $(n=7)$. The monocytes were primed with IFN- $\gamma$ and treated with GA in the presence of LPS for 24 hours. The activation marker expression was assessed by flow cytometry and the cytokine production was measured by ELISA. Shown are the percentages of subjects ( $\%$ from the group) in seven principal patterns. Data were collected on flow cytometry using the gating strategy in appendix 9 .

An HCA analysis of in vitro IC-treated monocytes led to the formation of eight different patterns of type II activation of monocytes (Figure 6.8). The eight patterns had from minimal to high mixture of groups. For example, while in pattern 4 a similar percent of subjects from all three groups was included, pattern 7 had only healthy subjects. The intensely mixed populations of subjects, clustered into all other patterns, indicate a strong variability of monocyte response to IC from subject to subject (Table 6.5). This is possibly due to individual variation, and less related to the three clinical groups of the subjects, i.e. healthy, non-GA treated MS and GA-treated MS groups. Finally, it may also indicate subgroups that are more or less responsive to IC treatment such as pattern 4 (least responsive) and patterns 3 and 8 (most responsive).

In summary, the HCA analysis of individual subjects, depending on phenotypes of unstimulated and in vitro LPS-stimulated monocytes, revealed a similarity between healthy and GA-treated MS groups which mostly fell into one pattern; whereas, non-GA treated MS patients were categorized into one distinct pattern. This finding indicates that the activation state of inflammatory monocytes is similar in healthy and GA-treated groups, but not in nonGA treated MS groups. 


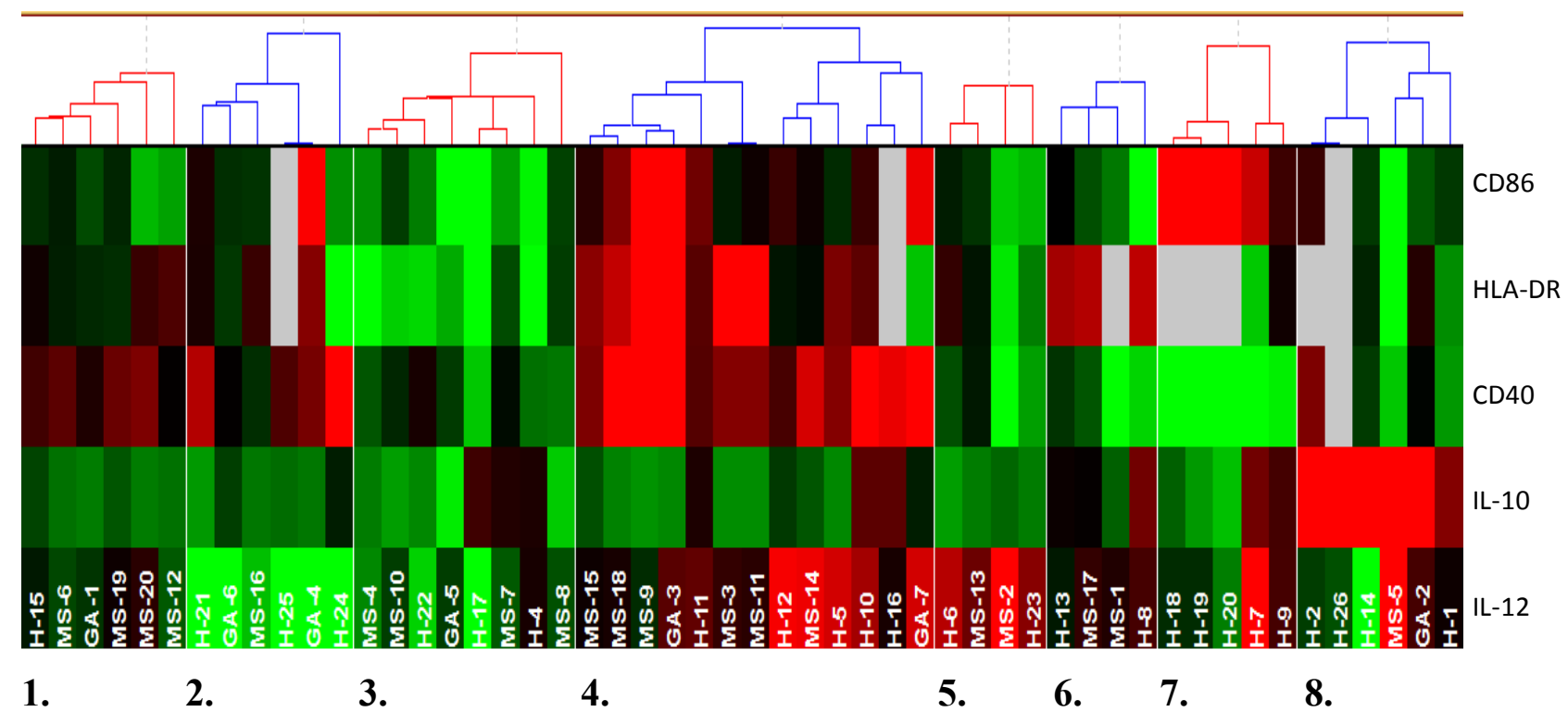

Figure 6.8. HCA dendrogram and heat map of in vitro IC-treated total monocytes.

Total monocytes were isolated from healthy subjects $(n=25)$, non-GA treated MS patients $(n=20)$ and GAtreated MS patients groups $(n=7)$. The monocytes were primed with IFN- $\gamma$ and treated with IC in the presence of LPS for 24 hours. The activation marker expression was assessed by flow cytometry and the cytokine production was measured by ELISA. The MFI values of activation markers have been analyzed in this study; whereas, the proportion of monocyte subsets has been assessed as a percentage of total monocyte population. Shown are the means and SD from individual experiments. (1) Pattern 1. (2) Pattern 2. (3) Pattern 3. (4) Pattern 4. (5) Pattern 5. (6) Pattern 6. (7) Pattern 7. (8) Pattern 8. Green color shows low expression, black - medium expression, red - high expression. The lowest expression is shown in dark green and the highest in dark red. Dendrogram represents the same data as in the figure 6.4. Data were collected on flow cytometry using the gating strategy in appendix 9.

\begin{tabular}{|l|l|l|l|l|l|l|l|l|l|}
\hline Groups & $\begin{array}{l}\text { Pattern } \\
1\end{array}$ & Pattern 2 & $\begin{array}{l}\text { Pattern } \\
3\end{array}$ & $\begin{array}{l}\text { Pattern } \\
4\end{array}$ & $\begin{array}{l}\text { Pattern } \\
5\end{array}$ & $\begin{array}{l}\text { Pattern } \\
6\end{array}$ & $\begin{array}{l}\text { Pattern } \\
7\end{array}$ & $\begin{array}{l}\text { Pattern } \\
8\end{array}$ & $\begin{array}{l}\text { Total } \\
\text { Non-GA treated MS }\end{array}$ \\
$4(21 \%)$ & $1(5 \%)$ & $3(16 \%)$ & $6(32 \%)$ & $2(11 \%)$ & $2(11 \%)$ & $0(0 \%)$ & $1(5 \%)$ & $\mathbf{2 0}(\mathbf{1 0 0} \%)$ \\
\hline GA-treated MS & $1(14 \%)$ & $2(29 \%)$ & $1(14 \%)$ & $2(29 \%)$ & $0(0 \%)$ & $0(0 \%)$ & $0(0 \%)$ & $1(14 \%)$ & $\mathbf{7 ( 1 0 0 \% )}$ \\
\hline Healthy & $1(4 \%)$ & $3(12 \%)$ & $3(12 \%)$ & $5(20 \%)$ & $2(5 \%)$ & $2(8 \%)$ & $5(20 \%)$ & $4(16 \%)$ & $\mathbf{2 5}(\mathbf{1 0 0} \%)$ \\
\hline
\end{tabular}

Table 6.5. HCA of in vitro IC-treated total monocytes.

Total monocytes were isolated from healthy subjects $(n=25)$, non-GA-treated MS patients $(n=20)$ and GAtreated MS patients $(n=7)$. The monocytes were primed with IFN- $\gamma$ and treated with IC in the presence of LPS for 24 hours. The activation marker expression was assessed by flow cytometry, and cytokine production was measured by ELISA. Shown are the percentages of subjects (\% from the group) in the eight major patterns. Data were collected on flow cytometry using the gating strategy in appendix 9. 


\subsection{DISCUSSION}

This chapter investigated the impact of GA treatment on monocytes in vivo. It demonstrated that in vivo GA-treated MS patients had a significant increase in CD16 monocytes; whereas, $\mathrm{CD} 14^{+}$monocytes were decreased in comparison to the non-GA treated MS patients. Interestingly, those $\mathrm{CD} 16^{+}$monocytes represent a population of type II activated monocytes in the GA-treated MS patients; whereas, in the non-GA treated patients they have the characteristics of classically activated cells. In addition, both subsets had been type II activated by in vivo GA treatment and the level of type II activation was greater in the monocytes exposed to GA in vivo than in monocytes exposed to GA in vitro. Finally, monocytes from healthy and MS subjects had specific patterns of response to GA that allowed them to form various groups. Together this study characterized for the first time the type II activation of total monocytes and monocyte subsets by in vivo GA treatment and the patterns of their response to GA.

\subsubsection{Proportion of monocytes from in vivo GA-treated MS patients}

In vivo GA treatment alters the proportion of monocyte subsets in MS after daily injections of $20 \mathrm{mg}$ GA subcutaneously. As previously described (Chapter 3), the CD14 monocytes are the major type of blood monocytes constituting $95 \%$ of total monocytes in healthy subjects and $74 \%$ in MS patients. However, the percentage of CD14 ${ }^{+}$monocytes is significantly lower in GA-treated patients and constituted only $57 \%$ percent of total monocytes. In parallel, the content of $\mathrm{CD} 16^{+}$monocytes significantly increased after in vivo GA treatment of MS patients (43\%), compared to healthy subjects' (5\%) and non-GA treated MS patients' groups (26\%).

Although there are no human studies that have reported the effect of GA on the proportion of monocyte subsets, Toker et al. have shown that the percentage of circulating $\mathrm{GA}^{+} \mathrm{CD} 16^{+}$monocytes time dependently decrease after the in vivo GA treatment in mouse model of MS, where the proportion of $\mathrm{GA}^{+} \mathrm{CD} 16^{+}$monocytes in the spleen and lymph nodes does not change. Although the high number of $\mathrm{CD} 16^{+}$monocytes remained during 18 hours after GA treatment, it dramatically dropped after 48 hours. This decrease in the number of $\mathrm{GA}^{+} \mathrm{CD} 16^{+}$monocytes was shown not to be due to their migration to the lymphoid organs[166]. Whether the $\mathrm{GA}^{+} \mathrm{CD} 16^{+}$monocytes migrated to the inflamed brain tissue, is yet 
to be elucidated. However, it is known that a ligand for CCR2 (CCL2) is detected at high levels in the brain lesions of MS patients[219-221] and both $\mathrm{CD}_{1} 4^{+}$and $\mathrm{CD} 16^{+}$monocytes express significantly increased chemokine receptor CCR2 (section 6.4.2), suggesting an increased migratory ability for the monocytes after the in vivo GA treatment in MS patients. Therefore, it is likely that the $\mathrm{GA}^{+}$monocytes detected at a high level within 18 hours of GA administration in the mouse model of MS[166] migrate to the inflamed brain tissue after 18 hours.

Toker et al. observed a high percentage of $\mathrm{GA}^{+} \mathrm{CD} 16^{+}$monocytes only within 18 hours of in vivo GA administration[166], where in this study, all seven MS patients had an increased proportion of $\mathrm{CD}^{+} 6^{+}$monocytes at various time points after the GA injection (data not shown). This finding indicates that the number of $\mathrm{CD} 16^{+}$monocytes remained high in the circulation of MS patients after the in vivo GA administration independently of the time of GA administration, unlike that seen in mice. On the other hand, the difference in proportion of $\mathrm{CD} 6^{+}$monocytes between humans and mice may be due to a prolonged effect of GA on human monocytes. As such, MS patients received daily repetitive doses of GA for a minimum of six months, where mice received a single dose of a GA injection. Since more than $95 \%$ of monocytes take up GA in vivo[166], it is possible that the high number of CD16 $6^{+}$ monocytes type II activated by GA (Section 6.4.2) is sustained through the replenishment from the $\mathrm{CD}_{1} 4^{+}$monocytes in MS patients given $\mathrm{CD}_{1} 6^{+}$monocytes are thought to be a differentiated form of $\mathrm{CD}_{1} 4^{+}$monocytes[77]. Since the in vitro GA treatment had a direct type II activating effect on both monocyte subsets (Chapter 5), this study further investigated whether these decreased $\mathrm{CD} 14^{+}$and increased $\mathrm{CD}_{1} 6^{+}$monocytes are in the state of type II activation after the in vivo GA-treatment of MS patients.

\subsubsection{In vivo GA effect on monocytes and their subsets}

While in vivo GA treatment has a type II activating effect on monocytes in a mouse model of MS, shown by a decrease in inflammatory cytokines IL-12 and TNF $\alpha$ and an increase in regulatory cytokine IL-10[43], there is not enough evidence to show the effect of in vivo GA treatment on human monocytes. This study demonstrated that GA type II activates human monocytes in MS not only in vitro (Chapters 4 and 5), but also in vivo, shown by a decreased expression of activation markers CD40, CD86, HLA-DR and CD64, without inhibiting their migration-related marker CCR2. This finding suggests that the in vivo 
type II activation by GA does not impair the ability of monocytes to migrate to the CNS in response to its ligand CCL2. In accordance with this study, Sellebjerg et al. have observed a decrease in the expression of only CD86 in monocytes from MS patients treated with GA in vivo. However, the expression of both CD40 and CD86 has been shown to be significantly downregulated in dendritic cells[218], which are known to be a differentiated form of monocytes[222]. Furthermore, the increased level of CD40 on dendritic cells correlates with a relapse risk in MS patients[218]. Together, these studies suggest that the downregulation of activation markers is an important mechanism for GA to prevent relapses in MS.

\subsubsection{Comparison of in vivo and in vitro GA effect on monocytes and their subsets}

In comparison to in vitro GA treatment, which only reduces the expression of CD40 and CD86 in the total monocytes but not their subsets and had no significant effect on other markers, the in vivo GA treatment significantly improves the effect on both subsets. This result indicates that $\mathrm{CD}_{1}{ }^{+}$and $\mathrm{CD}_{1} 6^{+}$monocytes of $\mathrm{MS}$ patients may require a more complex immune environment or a longer period of exposure in order to maximize their response to GA. GA also inhibits activation of other immune cells, such as dendritic cells and $\mathrm{T}$ cells, in $\mathrm{MS}[21,218]$. Therefore the enhanced immunomodulatory effect of GA on monocyte subsets, shown in this in vivo study, indicates a far more complex effect of GA on the immune system, in which $\mathrm{CD} 14^{+}$and $\mathrm{CD}_{1} 6^{+}$monocytes play an important part in MS patients. Overall, this comparative study investigating the in vivo and in vitro effect of GA suggests that in order to better understand and improve the effect of GA more emphasis should be placed on the in vivo interaction between various immune cells.

\subsubsection{Classical activation of in vivo GA-treated monocytes and their subsets}

Unlike monocytes from non-GA treated MS patients, in in vivo GA-treated monocytes classical activation was not induced by IFN- $\gamma$ priming followed by LPS stimulation. This result is in agreement with Kim et al., who have shown impaired classical activation of in vivo GA-treated monocytes with significantly lower IL-12 production after LPS stimulation[21]. , While Kim et al. shows that GA prevents induction of an inflammatory phenotype in monocytes of MS patients within 24 hours, in our study monocytes have been 
isolated 2-24 hours after the last GA injection. Our study suggests that GA not only type II activates monocytes, but also prevents activation of inflammatory monocytes in MS. This may be considered part of the mechanism of action of GA in prevention and reduction of MS relapse in the clinic[223].

\subsubsection{In vitro GA and IC effect on in vivo GA-treated monocytes and their subsets}

Although the expression of activation markers was not altered further by individual GA and IC treatments of in vivo GA-treated monocytes and their subsets, the production of IL-6 was significantly inhibited, whereas the production of IL-12 was inhibited only by GA. An interaction between the subsets is unlikely to be a cause for the low efficacy of in vitro GA treatment on in vivo GA-treated monocytes, as MS subsets maintained a good response to GA in co-cultures (Chapter 5). However, the low efficacy of IC might be due to a co-inhibition of the type II activation in monocyte subsets, as they have an impaired response to IC when cultured together. The possibility of GA pre-treatment to block the IC effect needs also to be considered, since down-regulation of a high affinity receptor for IC (CD64) by GA has been previously observed in monocytes (Chapter 4).

In comparison to GA and IC single treatments in vitro IC and GA combination treatment significantly enhanced the in vivo type II activating effect of GA on both subsets, suggesting that GA and IC combination treatment can be a rational approach to overcome the low responsiveness of in vivo GA-treated monocytes to GA or IC. However, IC would be beneficial if administered before or at the time of GA treatment in order to avoid the GAmediated downregulation of its receptor (CD64) having a negative effect on the response of monocytes to IC. Overall, the in vivo type II activating effect of GA on monocytes can be enhanced by additional GA and IC combination treatment, but not by individual administration of GA or IC.

Recently, combination treatments have been considered an effective therapeutic approach for MS[224]. Due to the complex mechanisms involved in the progression of MS, targeting several pathways may give additive benefits to the treatment of MS[208]. Although many different combinations have been tried[225, 226], a combination treatment of GA and IC has not been investigated so far. This study is the first to show that the combination of GA and IC 
treatments may be more beneficial in type II activation of monocytes, than the single treatments, and may improve the efficacy of in vivo GA treatment in MS patients.

\subsubsection{Comparison of monocyte activation between the subject groups}

Unsupervised hierarchical cluster analysis (HCA) is a powerful tool used for classification of patients by prognostic markers or response to therapy[227, 228]. In MS research HCA has been used for identification of distinct lesion groups[229, 230], or responders and non-responders to IFN- $\beta$ treatment[231]. In our study, HCA was used to cluster groups of subjects based on monocyte activation.

Three distinct groups of subjects can be defined depending on ex vivo monocyte activation state and monocyte subset proportion. Although the proportion of subsets differentiate the healthy and GA-treated MS groups, in general these two groups can be combined into one group, as both have relatively inactive monocytes. In contrast, monocytes from the non-GA treated MS groups showed a very different pattern of expression, which suggested a high state of classical activation and this was unique to this group. Therefore the in vivo GA treatment affects inflammatory monocytes in MS in a way that the monocytes obtain characteristics similar to that seen in healthy subjects.

HCA can be used for distinguishing a drug-responding group from non-responding group. This was shown by Axtell et al. where IFN- $\beta$ responding and non-responding MS patients were identified based on the production of inflammatory cytokines[231]. In our HCA study healthy subjects and MS patients were clustered into seven distinct groups by the activation state of in vitro GA-treated monocytes. Additionally GA-responders and nonresponders were seen to form distinct groups. Hence, HCA may be a valuable method to identify GA-responding and non-responding MS patients based on GA effect on the activation of monocytes. 


\subsection{CONCLUSION}

This study shows that despite the imbalance between monocyte subsets in in vivo GAtreated monocytes, both $\mathrm{CD} 14^{+}$and $\mathrm{CD}_{1} 6^{+}$monocyte subsets of the in vivo GA-treated MS patients showed characteristics of type II activation. In addition, in vivo GA treatment was shown to have a significantly stronger type II activating effect on monocytes and their subsets in MS patients, than the in vitro GA treatment. Further in vitro stimulation of in vivo GAtreated monocytes with LPS did not induce classical activation of the monocytes from MS patients; whereas, additional GA or IC treatments further reduced inflammatory cytokine production but not activation of marker expression. Finally, in vivo GA-induced type II activation of monocytes was further enhanced by GA and IC combination treatment, which may be a useful therapeutic approach for GA low-responding MS patients. 
Chapter 7.

General discussion 


\subsection{Outcome of results: the role of monocytes in the pathogenesis of MS}

Our study has provided important insight into the pathogenesis of MS by examining the alterations of monocytes and their subsets during MS. We found that circulating blood monocytes from MS patients had an inflammatory phenotype that was similar to that of classically activated monocytes (Chapter 3), especially with the CD16 ${ }^{+}$subset. In addition to their highly inflammatory nature, the $\mathrm{CD} 16^{+}$monocytes closely resembled the neurotoxic monocytes[97, 232] found in active[195] and early[33] demyelinating lesions of MS patients. This suggests that the $\mathrm{CD} 16^{+}$monocytes, but not the $\mathrm{CD} 14^{+}$monocytes, might play a key role in activating inflammatory $\mathrm{T}$ cells and directing nerve damage during MS. The role of $\mathrm{CD}_{16}{ }^{+}$monocytes in the disruption of the BBB is unknown; however, given that they can migrate through the $\mathrm{BBB}$ in vitro more effectively than lymphocytes[76] and $\mathrm{CD} 14^{+}$ monocytes[233], it is possible that $\mathrm{CD}^{+} 6^{+}$monocytes are one of the first inflammatory cells to infiltrate the CNS of MS patients.

$\mathrm{CD} 14^{+}$monocytes are unlikely to be directly involved in neuron damage, as they are not found in active lesions of MS, but rather only in the surrounding tissue[195]. In addition, a decrease in the number of $\mathrm{CD} 14^{+}$monocytes is negatively correlated with the Th1/Th2 cell ratio during $\mathrm{MS}$ [98]. Therefore, $\mathrm{CD} 14^{+}$monocytes do not appear to be responsible for the proliferation and activation of inflammatory Th1 and Th17 cells in MS. Our study indicates that $\mathrm{CD} 14^{+}$monocytes are less important in driving inflammation during MS, as $\mathrm{CD} 14^{+}$ monocytes from MS patients expressed only medium levels of inflammatory markers, compared to $\mathrm{CD}_{16} 6^{+}$monocytes (Chapter 3). Therefore, the role of $\mathrm{CD} 14^{+}$monocytes in MS is more likely to involve the removal or clearance of neuronal debris after the inflammation, rather than the induction of inflammation. In addition, $\mathrm{CD} 14^{+}$monocytes might play a regulatory role, as our study showed the $\mathrm{CD} 14^{+}$subset to be the main producer of IL-10, whereas the $\mathrm{CD}_{16}{ }^{+}$subset was the main producer of IL-12 (Chapter 5). It is also possible that $\mathrm{CD}_{1} 4^{+}$monocytes are involved in the process of regeneration (remyelinization) of neurons, as monocytes are capable of producing neurotrophic factors[234] and it has been suggested that $\mathrm{CD} 14^{+}$monocytes are involved in the transition from relapse to remission[98].

This study provides new knowledge about the co-regulation of monocyte subsets in healthy subjects (Chapter 5). Although the subsets can co-inhibit their activation in healthy subjects, $\mathrm{CD} 14^{+}$and $\mathrm{CD} 16^{+}$monocytes from MS patients failed to show an inhibitory effect on each other. These findings suggest that another mechanism of immune dysregulation in 
MS may result from the insufficient control of activation of $\mathrm{CD} 16^{+}$monocytes by $\mathrm{CD} 14^{+}$ monocytes in the periphery, leading to expansion and activation of this highly inflammatory subset, and their subsequent migration into the CNS. Our study significantly expands the knowledge of the immune mechanisms underlying MS by more fully elucidating the important role of monocytes. Our work has also been the first to characterize the distinct properties of the $\mathrm{CD} 14^{+}$and $\mathrm{CD} 16^{+}$monocyte subsets in GA-treated and non-GA treated MS patients. 


\subsection{Clinical significance and outcome of results: GA effect in MS}

Despite all of the evidence for an immunoregulatory effect of GA on the immune system in MS[218], little is known about the direct effect of GA on monocytes. Our study is the first to describe a direct type II activating effect of GA on monocytes, and the individual CD14 ${ }^{+}$ and $\mathrm{CD}_{1} 6^{+}$subsets[21]. Although GA can type II activate the total monocyte population (Chapter 4), this effect was not prominent in the CD14 $4^{+}$monocytes (Chapter 5). Instead, GA mainly affected the $\mathrm{CD}_{1} 6^{+}$monocytes in MS patients (Chapter 5), and significantly inhibited activation of this highly inflammatory subset (Chapter 3). In contrast to the inflammatory $\mathrm{CD}_{14}{ }^{+}$and $\mathrm{CD} 16^{+}$subsets that lost their ability to co-inhibit each other in untreated MS patients, GA-treatment of $\mathrm{CD} 16^{+}$monocytes from MS patients increased the type II activation of $\mathrm{CD}_{14}^{+}$monocytes by GA (Chapter 5). These results suggest that the type II activating effect of GA on $\mathrm{CD} 14^{+}$monocytes may be mediated by $\mathrm{CD} 16^{+}$monocytes, emphasizing an important role of $\mathrm{CD}^{+} 6^{+}$monocytes in regulating both classically activated and type II activated CD14 $4^{+}$monocytes in MS. It is also possible that other immune cells, such as T cells and dendritic cells, may be involved in mediating the GA effect on CD14 ${ }^{+}$ monocytes, as GA also modulates activation of T cells and dendritic cells[21, 218]. It is clear that GA has a complex effect on the immune system during MS, which merits further research particularly in this complex environment as suggested by our study, which showed that the interaction between different monocyte subsets can significantly affect the outcome of the treatment. While GA affected the activation and interaction of monocyte subsets, it did not inhibit a migration-related marker, CCR2, in total monocytes or either subset (Chapters

4 and 5), most likely preserving the ability of monocytes to migrate to the inflamed brain tissue.

Our HCA study showed that, despite a difference in the ratio between the subsets, in vivo GA-treated monocyte subsets had similar phenotypes to those from healthy subjects, and were distinct from the non-GA treated MS group monocytes (Chapter 6). Although both in vivo (Chapter 6) and in vitro (Chapters 4 and 5) GA treatments proved to be effective in type II activation of monocytes, GA is known to have only limited efficacy in MS (up to $30 \%[156])$, possibly as a result of its complex mechanism of action on immune cells. The recently used subcutaneous injection method of GA treatment to MS patients can result in uptake of GA by other resident immune cells of monocytic lineage, such as Langerhans cells[235] and dendritic cells[236] residing in the skin. This uptake may interfere with the 
transport of GA from the skin to the circulation, and could be one of the reasons for the limited efficacy of GA. Administering GA into the peripheral circulation, where it is targeted to encounter the maximal number of monocytes, could potentially improve its efficacy. GA efficacy might also be increased by combining it with other treatments. In particular, therapies that target $\mathrm{CD} 14^{+}$monocytes might have a synergistic effect, as GA failed to increase the number of these cells to a normal level (Chapter 6) and had a greater regulatory effect on the inflammatory $\mathrm{CD}_{16} 6^{+}$monocytes (Chapter 5). Overall, this study clearly describes a type II activating effect of GA on monocytes, and is the first study to explore how GA influences the individual monocyte subsets in MS. 


\subsection{Clinical significance and outcome of results: IC effect in MS}

To investigate a possible mechanism through which IVIG may be effective as a treatment for many autoimmune diseases[177-179], IC made from IVIG and RBC were used for the in vitro treatment of human monocytes. This study demonstrated that the IC had a direct type II activating effect on total monocytes (Chapter 4) and the individual subsets (Chapter 5) from MS patients. However, unlike GA, IC did not block the co-inhibitory effect of $\mathrm{CD} 14^{+}$and $\mathrm{CD} 16^{+}$subsets, and an interaction between the two subsets limited the type II activating effect of IC in vitro (Chapter 5). Previous studies have shown that IVIG has an immunomodulatory effect on numerous types of immune cells in vivo[202, 237, 238] and inhibits migration of inflammatory monocytes to the CNS in mice [238]. These findings indicate the complexity of the IVIG effect on the immune system. Because other immune cells can alter the effect that IC have on monocytes, further in vivo studies are needed in order to fully understand the complex role of IC on the immune response.

Finally, a combination of IC and GA treatment may be an alternative approach to treat MS, as this study showed that this type of combination treatment improved the level of type II activation, compared to single GA or IC treatments (Chapter 4). In clinics, however, the order of GA and IC treatments needs to be carefully considered, since in vitro IC treatment of in vivo GA pre-treated monocytes did not show any additional benefit (Chapter 6). Therefore, treatment with IC, followed by the treatment with GA might be an effective approach to treat MS. 


\subsection{Future directions}

Due to limitations in the availability of patients, our study mainly involved patients with RRMS; however, in order to further investigate the activation of monocytes in the different types of MS, including PPMS and SPMS, a higher number of patients will need to be recruited in future work. It may also be of benefit to examine the activation of monocyte subsets during both relapses and remissions of RRMS.

The ex vivo cytokine production in monocytes from MS patients was not examined, as isolated monocytes were given an additional in vitro stimulation with IFN- $\gamma$ and LPS to reach the level of cytokines that were detectable by ELISA, CBA and intracellular staining methods. As detection of cytokines in ex vivo isolated monocytes can be important in order to evaluate the in vivo GA and IC effects on monocytes, a more highly sensitive method, such as Enzyme-linked Immunospot Assay (ELISPOT)[239, 240] or RT-PCR could be used in future clinical studies.

In the in vivo GA-treated MS patients in this study, the monocytes were assessed only after the treatment. In order to assess how monocytes from particular MS patients are altered by the treatment, a comparison study of ex vivo isolated monocytes pre- and post-GA treatment could be conducted in the future.

Although this study demonstrated a type II activating effect of GA on monocytes both in vitro and in vivo, the IC effect was only shown in vitro. Therefore, the in vivo IC effect on monocytes needs to be further explored to understand the full picture of how IC affects monocytes. This work could be facilitated by studying the expression of activation markers and cytokines in ex vivo isolated monocytes from IVIG-treated patients. Alternatively, as IVIG and IC may affect monocytes through different pathways[241], treatment of MS patients with IC could be a potential treatment option for IVIG-resistant MS patients. In addition, to have a better idea about how monocytes from the same MS patients respond to IVIG and IC, activation of monocytes can be assessed both before and after the treatments. As IC may be naturally formed by binding of IVIG to A and B antigens on RBC (blood groups $\mathrm{A}$ and $\mathrm{B}$ ), the response of monocytes to in vivo IVIG treatment in MS patients with different $\mathrm{ABO}$ blood groups could be further investigated. 


\section{References}


1. Lucchinetti C, Parisi J, Scheithauer B, and Rodriguez M. Heterogeneity of multiple sclerosis lesions: implications for the pathogenesis of demyelination. Annals of neurology. 2000. 47(6): p. 707-717.

2. Zhang J, Markovic-Plese S, Lacet B, Raus J, Weiner HL, and Hafler DA. Increased frequency of interleukin 2-responsive $T$ cells specific for myelin basic protein and proteolipid protein in peripheral blood and cerebrospinal fluid of patients with multiple sclerosis. J Exp Med. 1994. 179(3): p. 973-84.

3. Lutton JD, Winston R, and Rodman TC. Multiple sclerosis: etiological mechanisms and future directions. Exp Biol Med (Maywood). 2004. 229(1): p. 12-20.

4. Neath J, Roessler RT, McMahon BT, and Rumrill PD. Patterns in perceived employment discrimination for adults with multiple sclerosis. Work. 2007. 29(3): p. 255-74.

5. Willer CJ, Dyment DA, Risch NJ, Sadovnick AD, Ebers GC, and Group TCCS. Twin concordance and sibling recurrence rates in multiple sclerosis. Proceedings of the National Academy of Sciences. 2003. 100(22): p. 12877-12882.

6. Poser S, Raun NE, and Poser W. Age at onset, initial symptomatology and the course of multiple sclerosis. Acta Neurologica Scandinavica. 1982. 66(3): p. 355-362.

7. Banwell B, Krupp L, Kennedy J, Tellier R, Tenembaum S, Ness J, Belman A, Boiko A, Bykova O, Waubant E, Mah JK, Stoian C, Kremenchutzky M, Bardini MR, Ruggieri M, Rensel M, Hahn J, Weinstock-Guttman B, Yeh EA, Farrell K, Freedman M, Iivanainen M, Sevon M, Bhan V, Dilenge M-E, Stephens D, and Bar-Or A. Clinical features and viral serologies in children with multiple sclerosis: a multinational observational study. The Lancet Neurology. 2007. 6(9): p. 773-781.

8. Rosati G. The prevalence of multiple sclerosis in the world: an update. Neurological Sciences. 2001. 22(2): p. 117-139.

9. Taylor BV, Pearson JF, Clarke G, Mason DF, Abernethy DA, Willoughby E, and Sabel C. MS prevalence in New Zealand, an ethnically and latitudinally diverse country. Multiple Sclerosis. 2010. 16(12): p. 1422-1431.

10. Sospedra M and Martin R. Immunology of multiple sclerosis. Annu Rev Immunol. 2005. 23: p. 683-747.

11. Barnett MH and Prineas JW. Relapsing and remitting multiple sclerosis: pathology of the newly forming lesion. Annals of neurology. 2004. 55(4): p. 458-468.

12. Thompson AJ, Kermode A, Wicks D, MacManus D, Kendall B, Kingsley D, and McDonald W. Major differences in the dynamics of primary and secondary progressive multiple sclerosis. Annals of neurology. 2004. 29(1): p. 53-62.

13. Ebers GC. Environmental factors and multiple sclerosis. The Lancet Neurology. 2008. 7(3): p. 268-277.

14. Dyment DA and Ebers GC, Sadovnick DA. Genetics of multiple sclerosis. The Lancet Neurology. 2004. 3(2): p. 104-110. 
15. Gilden DH. Infectious causes of multiple sclerosis. The Lancet Neurology. 2005. 4(3): p. 195-202.

16. Munger K1 LL, Hollis BW, Howard NS and Ascherio A. Serum 25-hydroxyvitamin D levels and risk of multiple sclerosis. JAMA. 2006. 296(23): p. 2832-2838.

17. Riise T, Nortvedt MW, and Ascherio A. Smoking is a risk factor for multiple sclerosis. Neurology. 2003. 61(8): p. 1122-1124.

18. Lauer K. Diet and multiple sclerosis. Neurology. 1997. 49(2 Suppl 2): p. S55-S61.

19. Bernard CCA and de Rosbo NK. Multiple sclerosis: an autoimmune disease of multifactorial etiology. Current Opinion in Immunology. 1992. 4(6): p. 760-765.

20. Racke MK, Lovett-Racke AE, and Karandikar NJ. The mechanism of action of glatiramer acetate treatment in multiple sclerosis. Neurology. 2010. 74(1 Supplement 1): p. S25-S30.

21. Kim HJ, Ifergan I, Antel JP, Seguin R, Duddy M, Lapierre Y, Jalili F, and Bar-Or A. Type 2 monocyte and microglia differentiation mediated by glatiramer acetate therapy in patients with multiple sclerosis. The Journal of Immunology. 2004. 172(11): p. 7144-7153.

22. Mosmann TR, Cherwinski H, Bond MW, Giedlin MA, and Coffman RL. Two types of murine helper T cell clone. I. Definition according to profiles of lymphokine activities and secreted proteins. J Immunol. 1986. 136(7): p. 2348-57.

23. Larsen M, Arnaud L, Hié M, Parizot C, Dorgham K, Shoukry M, Kemula M, Barete S, Derai D, Sauce D, Amoura Z, Pène J, Yssel H, and Gorochov G. Multiparameter grouping delineates heterogeneous populations of human IL-17 and/or IL-22 T-cell producers that share antigen specificities with other T-cell subsets. European Journal of Immunology. 2011. 41(9): p. 2596-2605.

24. Bar-Or A. The immunology of multiple sclerosis. Seminars in Neurology. 2008. 28(1): p. 29-45.

25. Issazadeh S, Mustafa M, Ljungdahl Å, Höjeberg B, Dagerlind Å, Elde R, and Olsson T. Interferon $\gamma$, interleukin 4 and transforming growth factor $\beta$ in experimental autoimmune encephalomyelitis in lewis rats: Dynamics of cellular mrna expression in the central nervous system and lymphoid cells. Journal of Neuroscience Research. 1995. 40(5): p. 579-590.

26. Skulina C, Schmidt S, Dornmair K, Babbe H, Roers A, Rajewsky K, Wekerle H, Hohlfeld R, and Goebels N. Multiple sclerosis: Brain-infiltrating CD8+T cells persist as clonal expansions in the cerebrospinal fluid and blood. Proceedings of the National Academy of Sciences of the United States of America. 2004. 101(8): p. 2428-2433.

27. Christensen JR, Börnsen L, Ratzer R, Piehl F, Khademi M, Olsson T, Sørensen PS, and Sellebjerg F. Systemic Inflammation in Progressive Multiple Sclerosis Involves Follicular T-Helper, Th17- and Activated B-Cells and Correlates with Progression. PLoS ONE. 2013. 8(3): p. e57820. 
28. $\mathrm{Li} \mathrm{Y}, \mathrm{Chu} \mathrm{N}, \mathrm{Hu} \mathrm{A}$, Gran B, Rostami A, and Zhang GX. Increased IL-23p19 expression in multiple sclerosis lesions and its induction in microglia. Brain. 2007. 130(Pt 2): p. 490-501.

29. Ifergan I, Kébir H, Bernard M, Wosik K, Dodelet-Devillers A, Cayrol R, Arbour N, and Prat A. The blood-brain barrier induces differentiation of migrating monocytes into Th17-polarizing dendritic cells. Brain. 2008. 131(3): p. 785-799.

30. Mohamadzadeh M, Olson S, Kalina WV, Ruthel G, Demmin GL, Warfield KL, Bavari S, and Klaenhammer TR. Lactobacilli activate human dendritic cells that skew $T$ cells toward $T$ helper 1 polarization. Proceedings of the National Academy of Sciences of the United States of America. 2005. 102(8): p. 2880-2885.

31. Isakov N, Scholz W, and Altman A. Signal transduction and intracellular events in Tlymphocyte activation. Immunology Today. 1986. 7(9): p. 271-277.

32. Weber MS, Starck M, Wagenpfeil S, Meinl E, Hohlfeld R, and Farina C. Multiple sclerosis: glatiramer acetate inhibits monocyte reactivity in vitro and in vivo. Brain. 2004. 127(6): p. 1370-1378.

33. Trebst C, Sorensen TL, Kivisakk P, Cathcart MK, and et al. CCR1+CCR5+ mononuclear phagocytes accumulate in the central nervous system of patients with multiple sclerosis. The American Journal of Pathology. 2001. 159(5): p. 1701-10.

34. Gordon S and Taylor PR. Monocyte and macrophage heterogeneity. Nat Rev Immunol. 2005. 5(12): p. 953-64.

35. Ziegler-Heitbrock HW. Definition of human blood monocytes. Journal of Leukocyte Biology. 2000. 67(5): p. 603-6.

36. Mosser DM and Edwards JP. Exploring the full spectrum of macrophage activation. Nature Reviews Immunology. 2008. 8(12): p. 958-969.

37. Sunderkötter C, Nikolic T, Dillon MJ, van Rooijen N, Stehling M, Drevets DA, and Leenen PJ. Subpopulations of mouse blood monocytes differ in maturation stage and inflammatory response. The Journal of Immunology. 2004. 172(7): p. 4410-4417.

38. Auffray C, Sieweke MH, and Geissmann F. Blood monocytes: development, heterogeneity, and relationship with dendritic cells. Annual review of immunology. 2009. 27: p. 669-692.

39. Dentener M, Bazil V, Von Asmuth E, Ceska M, and Buurman W. Involvement of CD14 in lipopolysaccharide-induced tumor necrosis factor-alpha, IL-6 and IL-8 release by human monocytes and alveolar macrophages. The Journal of Immunology. 1993. 150(7): p. 2885-2891.

40. Weber C, Erl W, Weber KSC, and Weber PC. HMG-CoA Reductase Inhibitors Decrease CDI1b Expression and CD11b-Dependent Adhesion of Monocytes to Endothelium and Reduce Increased Adhesiveness of Monocytes Isolated From Patients With Hypercholesterolemia. Journal of the American College of Cardiology. 1997. 30(5): p. 1212-1217. 
41. Ingersoll MA, Spanbroek R, Lottaz C, Gautier EL, Frankenberger M, Hoffmann R, Lang R, Haniffa M, Collin M, Tacke F, Habenicht AJR, Ziegler-Heitbrock L, and Randolph GJ. Comparison of gene expression profiles between human and mouse monocyte subsets. Blood. 2009. 115(3): p. e10-19.

42. Geissmann F, Manz MG, Jung S, Sieweke MH, Merad M, and Ley K. Development of Monocytes, Macrophages, and Dendritic Cells. Science. 2010. 327(5966): p. 656-661.

43. Weber MS, Prod'homme T, Youssef S, Dunn SE, Rundle CD, Lee L, Patarroyo JC, Stüve O, Sobel RA, and Steinman L. Type II monocytes modulate T cell-mediated central nervous system autoimmune disease. Nature medicine. 2007. 13(8): p. 935943.

44. Tierney JB, Kharkrang M, and La Flamme AC. Type II-activated macrophages suppress the development of experimental autoimmune encephalomyelitis. Immunology and Cell Biology. 2008. 87(3): p. 235-240.

45. Mantovani A, Sica A, Sozzani S, Allavena P, Vecchi A, and Locati M. The chemokine system in diverse forms of macrophage activation and polarization. Trends in immunology. 2004. 25(12): p. 677-686.

46. Galkina E and Ley K. Immune and inflammatory mechanisms of atherosclerosis. Annual review of immunology. 2009. 27: p. 165.

47. Benveniste E. Role of cytokines in multiple sclerosis, autoimmune encephalitis, and other neurological disorders. Journal of Molecular Medicine. 1995: p. 195-215.

48. Merrill JE and Benveniste EN. Cytokines in inflammatory brain lesions: helpful and harmful. Trends in Neurosciences. 1996. 19(8): p. 331-338.

49. Gerritse K, Laman JD, Noelle RJ, Aruffo A, Ledbetter JA, Boersma W, and Claassen E. CD40-CD40 ligand interactions in experimental allergic encephalomyelitis and multiple sclerosis. Proceedings of the National Academy of Sciences. 1996. 93(6): p. 2499-2504.

50. Alderson M, Armitage R, Tough T, Strockbine L, Fanslow W, and Spriggs M. CD40 expression by human monocytes: regulation by cytokines and activation of monocytes by the ligand for CD40. The Journal of experimental medicine. 1993. 178(2): p. 669674.

51. Bracci-Laudiero L, Aloe L, Caroleo MC, Buanne P, Costa N, Starace G, and Lundeberg T. Endogenous NGF regulates CGRP expression in human monocytes, and affects HLA-DR and CD86 expression and IL-10 production. Blood. 2005. 106(10): p. 3507-3514.

52. Lenschow DJ, Walunas TL, and Bluestone JA. CD28/B7 system of $T$ cell costimulation. Annual Review of Immunology. 1996. 14(1): p. 233-258.

53. Reder AT, Genc K, Byskosh PV, and Porrini AM. Monocyte activation in multiple sclerosis. Multiple Sclerosis. 1998. 4(3): p. 162-168. 
54. Lassmann $\mathrm{H}$, Brück $\mathrm{W}$, and Lucchinetti C. Heterogeneity of multiple sclerosis pathogenesis: implications for diagnosis and therapy. Trends in Molecular Medicine. 2001. 7(3): p. 115-121.

55. Verreck FA, de Boer T, Langenberg DM, Hoeve MA, Kramer M, Vaisberg E, Kastelein R, Kolk A, de Waal-Malefyt R, and Ottenhoff TH. Human IL-23-producing type 1 macrophages promote but IL-10-producing type 2 macrophages subvert immunity to (myco) bacteria. Proceedings of the National Academy of Sciences of the United States of America. 2004. 101(13): p. 4560-4565.

56. Mikita J, Dubourdieu-Cassagno N, Deloire MS, Vekris A, Biran M, Raffard G, Brochet B, Canron M-H, Franconi J-M, and Boiziau C. Altered M1/M2 activation patterns of monocytes in severe relapsing experimental rat model of multiple sclerosis. Amelioration of clinical status by M2 activated monocyte administration. Multiple Sclerosis Journal. 2011. 17(1): p. 2-15.

57. Suttles J, Milhorn DM, Miller RW, Poe JC, Wahl LM, and Stout RD. CD40 Signaling of Monocyte Inflammatory Cytokine Synthesis through an ERK1/2-dependent Pathway: A TARGET OF INTERLEUKIN (IL)-4 AND IL-10 ANTI-INFLAMMATORY $A C T I O N$. Journal of Biological Chemistry. 1999. 274(9): p. 5835-5842.

58. Fleischer J, Soeth E, Reiling N, GRAGE - GRIEBENOW E, FLAD HD, and Ernst M. Differential expression and function of CD80 (B7 - 1) and CD86 (B7 - 2) on human peripheral blood monocytes. Immunology. 1996. 89(4): p. 592-598.

59. van Vugt M, Heijnen A, Capel P, Park S, Ra C, Saito T, Verbeek J, and van de Winkel J. FcR gamma-chain is essential for both surface expression and function of human Fc gamma RI (CD64) in vivo. Blood. 1996. 87(9): p. 3593-3599.

60. Han KH, Tangirala RK, Green SR, and Quehenberger O. Chemokine Receptor CCR2 Expression and Monocyte Chemoattractant Protein-1-Mediated Chemotaxis in Human Monocytes A Regulatory Role for Plasma LDL. Arteriosclerosis, thrombosis, and vascular biology. 1998. 18(12): p. 1983-1991.

61. Hirayama K, Matsushita S, Kikuchi I, Iuchi M, Ohta N, and Sasazuki T. HLA-DQ is epistatic to HLA-DR in controlling the immune response to schistosomal antigen in humans. Nature. 1987. 327(6121): p. 426-30.

62. Zhou L, Ivanov II, Spolski R, Min R, Shenderov K, Egawa T, Levy DE, Leonard WJ, and Littman DR. IL-6 programs TH-17 cell differentiation by promoting sequential engagement of the IL-21 and IL-23 pathways. Nature immunology. 2007. 8(9): p. 967-974.

63. Korn T, Bettelli E, Oukka M, and Kuchroo VK. IL-17 and Th17 Cells. Annual Review of Immunology. 2009. 27: p. 485-517.

64. de Waal Malefyt R, Yssel H, and de Vries JE. Direct effects of IL-10 on subsets of human CD4+ T cell clones and resting T cells. Specific inhibition of IL-2 production and proliferation. The Journal of Immunology. 1993. 150(11): p. 4754-4765. 
65. Gately MK, Renzetti LM, Magram J, Stern AS, Adorini L, Gubler U, and Presky DH, The interleukin-12/interleukin-12-receptor system: Role in normal and pathologic immune responses, 1998. p. 495-521.

66. Vogel DY, Vereyken EJ, Glim J, Heijnen PD, Moeton M, van der Valk P, Amor S, Teunissen C, van Horssen J, and Dijkstra C. Macrophages in inflammatory multiple sclerosis lesions have an intermediate activation status. Journal of Neuroinflammation. 2013. 10(1): p. 35.

67. Kouwenhoven M, Teleshova N, Ozenci V, Press R, and Link H. Monocytes in multiple sclerosis: phenotype and cytokine profile. J Neuroimmunol. 2001. 112(1-2): p. 197-205.

68. Mahad DJ and Ransohoff RM. The role of MCP-1 (CCL2) and CCR2 in multiple sclerosis and experimental autoimmune encephalomyelitis (EAE). Seminars in Immunology. 2003. 15(1): p. 23-32.

69. Rincón M, Anguita J, Nakamura T, Fikrig E, and Flavell RA. Interleukin (IL)-6 directs the differentiation of IL-4-producing CD4+ $T$ cells. The Journal of Experimental Medicine. 1997. 185(3): p. 461-470.

70. Medzhitov R and Janeway Jr CA. Innate immunity: impact on the adaptive immune response. Current Opinion in Immunology. 1997. 9(1): p. 4-9.

71. Grewal IS and Flavell RA. CD40 and CD154 in cell-mediated immunity. Annual Review of Immunology. 1998. 16: p. 111-135.

72. Mildner A, Mack M, Schmidt H, Brück W, Djukic M, Zabel MD, Hille A, Priller J, and Prinz M. CCR2+ Ly-6Chi monocytes are crucial for the effector phase of autoimmunity in the central nervous system. Brain. 2009. 132(9): p. 2487-2500.

73. Boullerne AI, Rodríguez JJ, Touil T, Brochet B, Schmidt S, Abrous ND, Le Moal M, Pua JR, Jensen MA, and Mayo W. Anti-S-nitrosocysteine antibodies are a predictive marker for demyelination in experimental autoimmune encephalomyelitis: implications for multiple sclerosis. Journal of Neuroscience 2002. 22(1): p. 123-132.

74. Van Der Veen RC and Roberts LJ. Contrasting roles for nitric oxide and peroxynitrite in the peroxidation of myelin lipids. Journal of Neuroimmunology. 1999. 95(1-2): p. $1-7$.

75. Mitrovic B, Ignarro LJ, Vinters HV, Akers MA, Schmid I, Uittenbogaart C, and Merrill JE. Nitric oxide induces necrotic but not apoptotic cell death in oligodendrocytes. Neuroscience. 1995. 65(2): p. 531-539.

76. Bar - Or A, Nuttall RK, Duddy M, Alter A, Kim HJ, Ifergan I, Pennington CJ, Bourgoin P, Edwards DR, and Yong VW. Analyses of all matrix metalloproteinase members in leukocytes emphasize monocytes as major inflammatory mediators in multiple sclerosis. Brain. 2003. 126(12): p. 2738-2749.

77. Zawada AM, Rogacev KS, Rotter B, Winter P, Marell R-R, Fliser D, and Heine GH. SuperSAGE evidence for CD14++CD16+ monocytes as a third monocyte subset. Blood. 2011. 118(12): p. e50-e61. 
78. Ziegler-Heitbrock HW, Fingerle G, Strobel M, Schraut W, Stelter F, Schutt C, Passlick B, and Pforte A. The novel subset of CD14+/CD16+ blood monocytes exhibits features of tissue macrophages. Eur J Immunol. 1993. 23(9): p. 2053-8.

79. Zimmermann HW, Seidler S, Nattermann J, Gassler N, Hellerbrand C, Zernecke A, Tischendorf JJ, Luedde T, Weiskirchen R, Trautwein C, and Tacke F. Functional contribution of elevated circulating and hepatic non-classical CD14CD16 monocytes to inflammation and human liver fibrosis. PLoS One. 2010. 5(6): p. e11049.

80. Sanchez-Torres C, Garcia-Romo GS, Cornejo-Cortes MA, Rivas-Carvalho A, and Sanchez-Schmitz G. CD16+ and CD16- human blood monocyte subsets differentiate in vitro to dendritic cells with different abilities to stimulate CD4 $T$ cells. Int Immunol. 2001. 13(12): p. 1571-81.

81. Mizuno K, Toma T, Tsukiji H, Okamoto H, Yamazaki H, Ohta K, Ohta K, Kasahara Y, Koizumi S, and Yachie A. Selective expansion of CD16highCCR2- subpopulation of circulating monocytes with preferential production of haem oxygenase (HO)-1 in response to acute inflammation. Clin Exp Immunol. 2005. 142(3): p. 461-70.

82. Ziegler-Heitbrock L. The CD14+CD16+ blood monocytes: their role in infection and inflammation. Journal of Leukocyte Biology. 2007. 81(3): p. 584-592.

83. Belge KU, Dayyani F, Horelt A, Siedlar M, Frankenberger M, Frankenberger B, Espevik T, and Ziegler-Heitbrock L. The proinflammatory CD14+CD16+DR++ monocytes are a major source of TNF. J Immunol. 2002. 168(7): p. 3536-42.

84. Wong K, Chen W, Balakrishnan T, Toh Y, Fink K, and al. e. Susceptibility and response of human blood monocyte subsets to primary dengue virus infection. PLoS ONE. 2012. 7(5): p. e36435.

85. Passlick B, Flieger D, and Ziegler-Heitbrock H. Identification and characterization of a novel monocyte subpopulation in human peripheral blood. Blood. 1989. 74(7): p. 2527-2534.

86. Baeten D, Boots AMH, Steenbakkers PGA, Elewaut D, Bos E, Verheijden GFM, Verbruggen G, Miltenburg AMM, Rijnders AWM, Veys EM, and Filip De K. Human cartilage gp-39+,CD16+ monocytes in peripheral blood and synovium: Correlation with joint destruction in rheumatoid arthritis. Arthritis \& Rheumatism. 2000. 43(6): p. 1233-1243.

87. Hepburn A, Mason J, and Davies K. Expression of Fcy and complement receptors on peripheral blood monocytes in systemic lupus erythematosus and rheumatoid arthritis. Rheumatology. 2004. 43(5): p. 547-554.

88. Rothe G, Gabriel H, Kovacs E, Klucken J, Stöhr J, Kindermann W, and Schmitz G. Peripheral blood mononuclear phagocyte subpopulations as cellular markers in hypercholesterolemia. Arteriosclerosis, thrombosis, and vascular biology. 1996. 16(12): p. 1437-1447.

89. Katayama K, Matsubara T, Fujiwara M, Koga M, and Furukawa S. CD14+ CD16+ monocyte subpopulation in Kawasaki disease. Clinical \& Experimental Immunology. 2001. 121(3): p. 566-570. 
90. Zhong H, Bao W, Li X, Miller A, Seery C, Haq N, Bussel J, and Yazdanbakhsh K. CD16+ monocytes control T-cell subset development in immune thrombocytopenia. Blood. 2012. 120(16): p. 3326-35.

91. Baeten D, Boots AM, Steenbakkers PG, Elewaut D, Bos E, Verheijden GF, Verbruggen G, Miltenburg AM, Rijnders AW, and Veys EM. Human cartilage gp $39+, C D 16+$ monocytes in peripheral blood and synovium: Correlation with joint destruction in rheumatoid arthritis. Arthritis \& Rheumatism. 2001. 43(6): p. 12331243.

92. Skrzeczynska J, Kobylarz K, Hartwich Z, Zembala M, and Pryjma J. CD14+CD16+ monocytes in the course of sepsis in neonates and small children: monitoring and functional studies. Scandinavian journal of immunology. 2002. 55(6): p. 629-638.

93. Horelt A, Belge KU, Steppich B, Prinz J, and Ziegler - Heitbrock L. The CD14+ CD16+ monocytes in erysipelas are expanded and show reduced cytokine production. European journal of immunology. 2002. 32(5): p. 1319-1327.

94. Katayama K, Matsubara T, Fujiwara M, Koga M, and Furukawa S. CD14+CD16+ monocyte subpopulation in Kawasaki disease. Clinical \& Experimental Immunology. 2000. 121(3): p. 566-570.

95. Fingerle R, Angstwurm, Andreesen, and Ziegler H. Selective depletion of CD14+CD16+ monocytes by glucocorticoid therapy. Clinical \& Experimental Immunology. 1998. 112(3): p. 501-506.

96. Wijngaarden S, Van Roon J, Bijlsma J, Van De Winkel J, and Lafeber F. Fcy receptor expression levels on monocytes are elevated in rheumatoid arthritis patients with high erythrocyte sedimentation rate who do not use anti - rheumatic drugs. Rheumatology. 2003. 42(5): p. 681-688.

97. Lopez-Moratalla N, Gonzalez A, Aymerich MS, Lopez-Zabalza MJ, Pio R, de Castro $\mathrm{P}$, and Santiago E. Monocyte inducible nitric oxide synthase in multiple sclerosis: regulatory role of nitric oxide. Nitric Oxide. 1997. 1(1): p. 95-104.

98. Nakajima H, Sugino M, Kimura F, Hanafusa T, Ikemoto T, and Shimizu A. Decreased CD14+CCR2+ monocytes in active multiple sclerosis. Neurosci Lett. 2004. 363(2): p. 187-9.

99. Iwahashi M, Yamamura M, Aita T, Okamoto A, Ueno A, Ogawa N, Akashi S, Miyake K, Godowski PJ, and Makino H. Expression of toll-like receptor 2 on CD16+ blood monocytes and synovial tissue macrophages in rheumatoid arthritis. Arthritis \& Rheumatism. 2004. 50(5): p. 1457-1467.

100. Dayyani F, Belge K-U, Frankenberger M, Mack M, Berki T, and Ziegler-Heitbrock L. Mechanism of glucocorticoid-induced depletion of human CD14+CD16+ monocytes. Journal of Leukocyte Biology. 2003. 74(1): p. 33-39.

101. Gartner S. HIV Infection and Dementia. Science. 2000. 287(5453): p. 602-604. 
102. Burdo T, Soulas C, Orzechowski K, Button J, Krishnan A, Sugimoto C, Alvarez X, Kuroda $\mathrm{M}$, and Williams $\mathrm{K}$. Increased monocyte turnover from bone marrow correlates with severity of SIV encephalitis and CD163 levels in plasma. PLoS Pathogens. 2010. 6(4): p. e1000842.

103. Fischer-Smith SC, Andrij E Sverstiuk, Christelle Capini, Darryl L'Heureux, Emmanuel G Régulier, Max W Richardson, Shohreh Amini, Susan Morgello, Kamel Khalili, Jay Rappaport, Tracy. CNS invasion by CD14+/CD16+ peripheral bloodderived monocytes in HIV dementia: perivascular accumulation and reservoir of HIV infection. Journal of Neurovirology. 2001. 7(6): p. 528-541.

104. Pulliam L, Gascon R, Stubblebine M, McGuire D, and McGrath MS. Unique monocyte subset in patients with AIDS dementia. The Lancet. 1997. 349(9053): p. 692-695.

105. Wijngaarden S, van Roon JAG, Bijlsma JWJ, van de Winkel JGJ, and Lafeber FPJG. Fcy receptor expression levels on monocytes are elevated in rheumatoid arthritis patients with high erythrocyte sedimentation rate who do not use anti-rheumatic drugs. Rheumatology. 2003. 42(5): p. 681-688.

106. Steven LG and Clyde M. US FDA-Approved Disease-Modifying Treatments for Multiple Sclerosis: Review of Adverse Effect Profiles. CNS Drugs. 2005. 19(3): p. 239-252.

107. Singer B, Ross A, and Tobias K. Oral fingolimod for the treatment of patients with relapsing forms of multiple sclerosis. International journal of clinical practice. 2011. 65(8): p. 887-895.

108. Steinman L. Blocking adhesion molecules as therapy for multiple sclerosis: natalizumab. Nature Reviews Drug Discovery. 2005. 4(6): p. 510-518.

109. Rizvi SA and Bashir K. Other therapy options and future strategies for treating patients with multiple sclerosis. Neurology. 2004. 63(12 suppl 6): p. S47-S54.

110. He D, Xu Z, Dong S, Zhang H, Zhou H, Wang L, and Zhang S. Teriflunomide for multiple sclerosis. Cochrane Database Syst Rev. 2012. 12: p. CD009882.

111. Bar-Or A, Gold R, Kappos L, Arnold DL, Giovannoni G, Selmaj K, O'Gorman J, Stephan M, and Dawson KT. Clinical efficacy of BG-12 (dimethyl fumarate) in patients with relapsing-remitting multiple sclerosis: subgroup analyses of the DEFINE study. J Neurol. 2013.

112. Rudick RA, Stuart WH, Calabresi PA, Confavreux C, Galetta SL, Radue E-W, Lublin FD, Weinstock-Guttman B, Wynn DR, and Lynn F. Natalizumab plus interferon betala for relapsing multiple sclerosis. New England Journal of Medicine. 2006. 354(9): p. 911-923.

113. DeAngelis T and Lublin F. Neurotherapeutics in multiple sclerosis: novel agents and emerging treatment strategies. Mount Sinai Journal of Medicine: A Journal of Translational and Personalized Medicine. 2008. 75(2): p. 157-167. 
114. Coles AJ, Cox A, Page E, Jones J, Trip SA, Deans J, Seaman S, Miller DH, Hale G, Waldmann $\mathrm{H}$, and Compston DA. The window of therapeutic opportunity in multiple sclerosis. Journal of Neurology. 2006. 253(1): p. 98-108.

115. Yong VW. Differential mechanisms of action of interferon- $\beta$ and glatiramer acetate in MS. Neurology. 2002. 59(6): p. 802-808.

116. Hartung H-P, Bar-Or A, and Zoukos Y. What do we know about the mechanism of action of disease-modifying treatments in MS? JOURNAL OF NEUROLOGYBERLIN-. 2004. 251: p. 12-29.

117. Hall GL, Wing MG, Compston DAS, and Scolding NJ. $\beta$-interferon regulates the immunomodulatory activity of neonatal rodent microglia. Journal of Neuroimmunology. 1997. 72(1): p. 11-19.

118. Giovannoni G, Munschauer FE, and Deisenhammer F. Neutralising antibodies to interferon beta during the treatment of multiple sclerosis. Journal of Neurology, Neurosurgery \& Psychiatry. 2002. 73(5): p. 465-469.

119. Walther EU and Hohlfeld R. Multiple sclerosis: Side effects of interferon beta therapy and their management. Neurology. 1999. 53(8): p. 1622.

120. Esposito F, Radaelli M, Martinelli V, Sormani MP, Martinelli Boneschi F, Moiola L, Rocca MA, Rodegher M, and Comi G. Comparative study of mitoxantrone efficacy profile in patients with relapsing-remitting and secondary progressive multiple sclerosis. Mult Scler. 2010. 16(12): p. 1490-9.

121. Chan A, Weilbach FX, Toyka KV, and Gold R. Mitoxantrone induces cell death in peripheral blood leucocytes of multiple sclerosis patients. Clinical \& Experimental Immunology. 2005. 139(1): p. 152-158.

122. Wiseman LR and Spencer CM. Mitoxantrone. A review of its pharmacology and clinical efficacy in the management of hormone-resistant advanced prostate cancer. Drugs \& aging. 1997. 10(6): p. 473-485.

123. Millefiorini E, Gasperini C, Pozzilli C, D'andrea F, Bastianello S, Trojano M, Morino S, Morra VB, Bozzao A, and Calo' A. Randomized placebo-controlled trial of mitoxantrone in relapsing-remitting multiple sclerosis: 24-month clinical and MRI outcome. Journal of neurology. 1997. 244(3): p. 153-159.

124. Kingwell E, Koch M, Leung B, Isserow S, Geddes J, Rieckmann P, and Tremlett H. Cardiotoxicity and other adverse events associated with mitoxantrone treatment for MS. Neurology. 2010. 74(22): p. 1822-1826.

125. Kappos L, Antel J, Comi G, Montalban X, O'Connor P, Polman CH, Haas T, Korn AA, Karlsson G, and Radue EW. Oral Fingolimod (FTY720) for Relapsing Multiple Sclerosis. New England Journal of Medicine. 2006. 355(11): p. 1124-1140.

126. Seki N, Maeda Y, Kataoka H, Sugahara K, Sugita T, and Chiba K. Fingolimod (FTY720) ameliorates experimental autoimmune encephalomyelitis (EAE). II. FTY720 decreases infiltration of Th17 and Th1 cells into the central nervous system in EAE. Inflammation and Regeneration. 2010.30(6): p. 542-548. 
127. Cohen JA, Barkhof F, Comi G, Hartung H-P, Khatri BO, Montalban X, Pelletier J, Capra R, Gallo P, Izquierdo G, Tiel-Wilck K, de Vera A, Jin J, Stites T, Wu S, Aradhye S, and Kappos L. Oral Fingolimod or Intramuscular Interferon for Relapsing Multiple Sclerosis. New England Journal of Medicine. 2010. 362(5): p. 402-415.

128. Yousry TA, Major EO, Ryschkewitsch C, Fahle G, Fischer S, Hou J, Curfman B, Miszkiel K, Mueller-Lenke N, Sanchez E, Barkhof F, Radue E-W, Jäger HR, and Clifford DB. Evaluation of Patients Treated with Natalizumab for Progressive Multifocal Leukoencephalopathy. New England Journal of Medicine. 2006. 354(9): p. 924-933.

129. Tan I, McArthur J, Clifford D, Major E, and Nath A. Immune reconstitution inflammatory syndrome in natalizumab-associated PML. Neurology. 2011. 77(11): p. 1061-1067.

130. Tischner D and Reichardt HM. Glucocorticoids in the control of neuroinflammation. Molecular and Cellular Endocrinology. 2007. 275(1-2): p. 62-70.

131. Milligan N, Newcombe R, and Compston D. A double-blind controlled trial of high dose methylprednisolone in patients with multiple sclerosis: 1. Clinical effects. Journal of Neurology, Neurosurgery \& Psychiatry. 1987. 50(5): p. 511-516.

132. Elenkov IJ and Chrousos GP. Stress hormones, Th1/Th2 patterns, pro/antiinflammatory cytokines and susceptibility to disease. Trends in Endocrinology \& Metabolism. 1999. 10(9): p. 359-368.

133. Herold M, McPherson K, and Reichardt H. Glucocorticoids in T cell apoptosis and function. Cellular and molecular life sciences. 2006. 63(1): p. 60-72.

134. Tuckermann JP, Kleiman A, Moriggl R, Spanbroek R, Neumann A, Illing A, Clausen $\mathrm{BE}$, Stride B, Forster I, and Habenicht AJ. Macrophages and neutrophils are the targets for immune suppression by glucocorticoids in contact allergy. Journal of Clinical Investigation. 2007. 117(5): p. 1381.

135. Wüst S, van den Brandt J, Tischner D, Kleiman A, Tuckermann JP, Gold R, Lühder F, and Reichardt HM. Peripheral T cells are the therapeutic targets of glucocorticoids in experimental autoimmune encephalomyelitis. The Journal of Immunology. 2008. 180(12): p. 8434-8443.

136. Greenwood J, Etienne-Manneville S, Adamson P, and Couraud P-O. Lymphocyte migration into the central nervous system: implication of ICAM-1 signalling at the blood-brain barrier. Vascular pharmacology. 2002. 38(6): p. 315-322.

137. Correale J and Villa A. The blood-brain-barrier in multiple sclerosis: functional roles and therapeutic targeting. Autoimmunity. 2007. 40(2): p. 148-160.

138. Pozzilli C, Marinelli F, Romano S, and Bagnato F. Corticosteroids treatment. Journal of the neurological sciences. 2004. 223(1): p. 47-51.

139. Benjamin RJ and Waldmann H. Induction of tolerance by monoclonal antibody therapy. 1986. 
140. Coles AJ, Wing MG, Molyneux P, Paolillo A, Davie CM, Hale G, Miller D, Waldmann $\mathrm{H}$, and Compston A. Monoclonal antibody treatment exposes three mechanisms underlying the clinical course of multiple sclerosis. Annals of neurology. 1999. 46(3): p. 296-304.

141. Bielekova B and Becker BL. Monoclonal antibodies in MS: Mechanisms of action. Neurology. 2010. 74(1 Supplement 1): p. S31-S40.

142. Cuker A, Coles AJ, Sullivan H, Fox E, Goldberg M, Oyuela P, Purvis A, Beardsley DS, and Margolin DH. A distinctive form of immune thrombocytopenia in a phase 2 study of alemtuzumab for the treatment of relapsing-remitting multiple sclerosis. Blood. 2011. 118(24): p. 6299-6305.

143. Osterborg A, Dyer M, Bunjes D, Pangalis GA, Bastion Y, Catovsky D, and Mellstedt H. Phase II multicenter study of human CD52 antibody in previously treated chronic lymphocytic leukemia. European Study Group of CAMPATH-1H Treatment in Chronic Lymphocytic Leukemia. Journal of Clinical Oncology. 1997. 15(4): p. 15671574.

144. Seite J-F, Shoenfeld Y, Youinou P, and Hillion S. What is the contents of the magic draft IVIg? Autoimmunity Reviews. 2008. 7(6): p. 435-439.

145. Duhem C, Dicato MA, and Ries F. Side-effects of intravenous immune globulins. Clin Exp Immunol. 1994. 97 Suppl 1: p. 79-83.

146. Fazekas F, Deisenhammer F, Strasser-Fuchs S, Nahler G, and Mamoli B. Randomised placebo-controlled trial of monthly intravenous immunoglobulin therapy in relapsingremitting multiple sclerosis. The Lancet. 1997. 349(9052): p. 589-593.

147. Achiron A, Gabbay U, Gilad R, Hassin-Baer S, Barak Y, Gornish M, Elizur A, Goldhammer Y, and Sarova-Pinhas I. Intravenous immunoglobulin treatment in multiple sclerosis. Effect on relapses. Neurology. 1998. 50(2): p. 398-402.

148. Sorensen P, Wanscher B, Jensen C, Schreiber K, Blinkenberg M, Ravnborg M, Kirsmeier $\mathrm{H}$, Larsen V, and Lee M. Intravenous immunoglobulin $G$ reduces MRI activity in relapsing multiple sclerosis. Neurology. 1998. 50(5): p. 1273-1281.

149. Lewańska M, Siger-Zajdel M, and Selmaj K. No difference in efficacy of two different doses of intravenous immunoglobulins in MS: clinical and MRI assessment. European Journal of Neurology. 2002. 9(6): p. 565-572.

150. Bayry J, Lacroix-Desmazes S, Carbonneil C, Misra N, Donkova V, Pashov A, Chevailler A, Mouthon L, Weill B, Bruneval P, Kazatchkine MD, and Kaveri SV. Inhibition of maturation and function of dendritic cells by intravenous immunoglobulin. Blood. 2003. 101(2): p. 758-765.

151. Reske D, Thomas AV, Petereit HF, Fink GR, and Schroeter M. Impact of immunomodulatory treatment on leukocyte cytokine production in multiple sclerosis patients and healthy donors. Neuroimmunomodulation. 2009. 16(6): p. 385-91.

152. Bruneau JM, Yea CM, Spinella-Jaegle S, Fudali C, Woodward K, Robson PA, Sautes C, Westwood R, Kuo EA, Williamson RA, and Ruuth E. Purification of human 
dihydro-orotate dehydrogenase and its inhibition by A77 1726, the active metabolite of leflunomide. Biochem J. 1998. 336 ( Pt 2): p. 299-303.

153. Linker RA, Lee D-H, Ryan S, van Dam AM, Conrad R, Bista P, Zeng W, Hronowsky X, Buko A, Chollate S, Ellrichmann G, Brück W, Dawson K, Goelz S, Wiese S, Scannevin RH, Lukashev M, and Gold R. Fumaric acid esters exert neuroprotective effects in neuroinflammation via activation of the Nrf2 antioxidant pathway. Brain. 2011. 134(3): p. 678-692.

154. Hutchinson M, Fox RJ, Miller DH, Phillips JT, Kita M, Havrdova E, O'Gorman J, Zhang R, Novas M, Viglietta V, and Dawson KT. Clinical efficacy of BG-12 (dimethyl fumarate) in patients with relapsing-remitting multiple sclerosis: subgroup analyses of the CONFIRM study. J Neurol. 2013.

155. Noyes K, Bajorska A, Chappel A, Schwid SR, Mehta LR, Weinstock-Guttman B, Holloway RG, and Dick AW. Cost-effectiveness of disease-modifying therapy for multiple sclerosis: A population-based study. Neurology. 2011. 77(4): p. 355-363.

156. Johnson K, Brooks B, Cohen J, Ford C, Goldstein J, Lisak R, Myers L, Panitch H, Rose J, and Schiffer R. Copolymer 1 reduces relapse rate and improves disability in relapsing - remitting multiple sclerosis Results of a phase III multicenter, double blind, placebo - controlled trial. Neurology. 1995. 45(7): p. 1268-1276.

157. Neuhaus O, Farina C, Wekerle H, and Hohlfeld R. Mechanisms of action of glatiramer acetate in multiple sclerosis. Neurology. 2001. 56(6): p. 702-708.

158. Farina C, Bergh FT, Albrecht H, Meinl E, Yassouridis A, Neuhaus O, and Hohlfeld R. Treatment of multiple sclerosis with Copaxone (COP) Elispot assay detects COPinduced interleukin-4 and interferon- $\gamma$ response in blood cells. Brain. 2001. 124(4): p. 705-719.

159. Miller A, Shapiro S, Gershtein R, Kinarty A, Rawashdeh H, Honigman S, and Lahat N. Treatment of multiple sclerosis with Copolymer-1 (Copaxone $\left.{ }^{\circledR}\right)$ : implicating mechanisms of Th1 to Th2/Th3 immune-deviation. Journal of neuroimmunology. 1998. 92(1): p. 113-121.

160. Aharoni R, Teitelbaum D, Leitner O, Meshorer A, Sela M, and Arnon R. Specific Th2 cells accumulate in the central nervous system of mice protected against experimental autoimmune encephalomyelitis by copolymer 1. Proceedings of the National Academy of Sciences. 2000. 97(21): p. 11472-11477.

161. Brenner T, Arnon R, Sela M, Abramsky O, Meiner Z, Riven-Kreitman R, Tarcik N, and Teitelbaum D. Humoral and cellular immune responses to Copolymer 1 in multiple sclerosis patients treated with Copaxone ${ }^{\circledR}$. Journal of neuroimmunology. 2001. 115(1): p. 152-160.

162. Teitelbaum D, Fridkis-Hareli M, Arnon R, and Sela M. Copolymer 1 inhibits chronic relapsing experimental allergic encephalomyelitis induced by proteolipid protein (PLP) peptides in mice and interferes with PLP-specific T cell responses. Journal of Neuroimmunology. 1996. 64(2): p. 209-217. 
163. Ben-Nun A, Mendel I, Bakimer R, Fridkis-Hareli M, Teitelbaum D, Arnon R, Sela M, and Kerlero de Rosbo N. The autoimmune reactivity to myelin oligodendrocyte glycoprotein (MOG) in multiple sclerosis is potentially pathogenic: effect of copolymer 1 on MOG-induced disease. Journal of neurology. 1996. 243: p. 14-22.

164. Fridkis-Hareli M, Teitelbaum D, Gurevich E, Pecht I, Brautbar C, Kwon OJ, Brenner $\mathrm{T}$, Arnon R, and Sela M. Direct binding of myelin basic protein and synthetic copolymer 1 to class II major histocompatibility complex molecules on living antigenpresenting cells--specificity and promiscuity. Proceedings of the National Academy of Sciences. 1994. 91(11): p. 4872-4876.

165. Ford C, Johnson K, Lisak R, Panitch H, Shifroni G, and Wolinsky J. A prospective open-label study of glatiramer acetate: over a decade of continuous use in multiple sclerosis patients. Multiple sclerosis. 2006. 12(3): p. 309-320.

166. Toker A, Slaney C, Bäckström B, and Harper J. Glatiramer Acetate Treatment Directly Targets CD11b+Ly6G- Monocytes and Enhances the Suppression of Autoreactive T cells in Experimental Autoimmune Encephalomyelitis. Scandinavian Journal of Immunology. 2011. 74(3): p. 235-243.

167. Wekerle H, Linington C, Lassmann H, and Meyermann R. Cellular immune reactivity within the CNS. Trends in Neurosciences. 1986. 9: p. 271-277.

168. Hickey WF. Migration of hematogenous cells through the blood - brain barrier and the initiation of CNS inflammation. Brain Pathology. 2008. 1(2): p. 97-105.

169. Pul R, Moharregh-Khiabani D, Skuljec J, Skripuletz T, Garde N, Voss EV, and Stangel M. Glatiramer acetate modulates TNF-alpha and IL-10 secretion in microglia and promotes their phagocytic activity. J Neuroimmune Pharmacol. 2011. 6(3): p. 381-8.

170. Pul R, Morbiducci F, Škuljec J, Skripuletz T, Singh V, Diederichs U, Garde N, Voss EV, Trebst C, and Stangel M. Glatiramer Acetate Increases Phagocytic Activity of Human Monocytes <italic $>$ In Vitro </italic $>$ and in Multiple Sclerosis Patients. PLoS ONE. 2012. 7(12): p. e51867.

171. Lloyd W and Schur PH. Immune complexes, complement, and anti-DNA in exacerbations of systemic lupus erythematosus (SLE). Medicine. 1981. 60(3): p. 208217.

172. Zvaifler NJ. Rheumatoid synovitis. An extravascular immune complex disease. Arthritis \& Rheumatism. 1974. 17(3): p. 297-305.

173. Gerber JS and Mosser DM. Reversing lipopolysaccharide toxicity by ligating the macrophage Fc gamma receptors. J Immunol. 2001. 166(11): p. 6861-8.

174. Knezevic-Maramica I and Kruskall MS. Intravenous immune globulins: an update for clinicians. Transfusion. 2003. 43(10): p. 1460-1480.

175. Kessary-Shoham H, Levy Y, Shoenfeld Y, Lorber M, and Gershon H. In vivoAdministration of Intravenous Immunoglobulin (IVIg) can Lead to Enhanced Erythrocyte Sequestration. Journal of Autoimmunity. 1999. 13(1): p. 129-135. 
176. Jurisdictional Blood Committee. Criteria for the clinical use of intravenous immunoglobulin in Australia. Health Minister's Conference. 2007.

177. Salama A, Mueller-Eckhardt C, and Kiefel V. Effect of intravenous immunoglobulin in immune thrombocytopenia: competitive inhibition of reticuloendothelial system function by sequestration of autologous red blood cells? The Lancet. 1983. 322(8343): p. 193-195.

178. Shrestha S, Wiener H, Olson AK, Edberg JC, Bowles NE, Patel H, and Portman MA. Functional FcyRIIB Gene Variants Influence Intravenous Immunoglobulin (IVIG) Response in Kawasaki Disease (KD) Patients. The Journal of Allergy and Clinical Immunology. 2011. 128(3): p. 677.

179. Hughes R. Systematic reviews of treatment for inflammatory demyelinating neuropathy*. Journal of Anatomy. 2002. 200(4): p. 331-339.

180. Ichiyama T, Ueno Y, Hasegawa M, Niimi A, Matsubara T, and Furukawa S. Intravenous immunoglobulin inhibits NF-kappaB activation and affects Fcgamma receptor expression in monocytes/macrophages. Naunyn Schmiedebergs Arch Pharmacol. 2004. 369(4): p. 428-33.

181. Kurtzke JF. Rating neurologic impairment in multiple sclerosis: an expanded disability status scale (EDSS). Neurology. 1983. 33(11): p. 1444-52.

182. Berridge MV, Herst PM, and Tan AS, Tetrazolium dyes as tools in cell biology: New insights into their cellular reduction, in Biotechnology Annual Review, El-Gewely M R, Editor. 2005, Elsevier. p. 127-152.

183. Schmittgen TD and Livak KJ. Analyzing real-time PCR data by the comparative CT method. Nature protocols. 2008. 3(6): p. 1101-1108.

184. Filion LG, Matusevicius D, Graziani-Bowering GM, Kumar A, and Freedman MS. Monocyte-derived IL12, CD86 (B7-2) and CD40L expression in relapsing and progressive multiple sclerosis. Clinical Immunology. 2003. 106(2): p. 127-138.

185. Kebir H, Ifergan I, Alvarez JI, Bernard M, Poirier J, Arbour N, Duquette P, and Prat A. Preferential recruitment of interferon- $\gamma$-expressing TH17 cells in multiple sclerosis. Annals of Neurology. 2009. 66(3): p. 390-402.

186. Nakayama F, Nishihara S, Iwasaki H, Kudo T, Okubo R, Kaneko M, Nakamura M, Karube M, Sasaki K, and Narimatsu H. CD15 Expression in Mature Granulocytes Is Determined by $\alpha 1,3-F u c o s y l t r a n s f e r a s e ~ I X$, but in Promyelocytes and Monocytes by a1,3-Fucosyltransferase IV. Journal of Biological Chemistry. 2001. 276(19): p. 16100-16106.

187. Poli A, Michel T, Thérésine M, Andrès E, Hentges F, and Zimmer J. CD56bright natural killer (NK) cells: an important NK cell subset. Immunology. 2009. 126(4): p. 458-465.

188. Chang TT, Jabs C, Sobel RA, Kuchroo VK, and Sharpe AH. Studies in B7-Deficient Mice Reveal a Critical Role for B7 Costimulation in Both Induction and Effector 
Phases of Experimental Autoimmune Encephalomyelitis. The Journal of Experimental Medicine. 1999. 190(5): p. 733-740.

189. Howard LM, Miga AJ, Vanderlugt CL, Dal Canto MC, Laman JD, Noelle RJ, and Miller SD. Mechanisms of immunotherapeutic intervention by anti-CD40L (CD154) antibody in an animal model of multiple sclerosis. J Clin Invest. 1999. 103(2): p. 28190.

190. Cross AH, Girard TJ, Giacoletto KS, Evans RJ, Keeling RM, Lin RF, Trotter JL, and Karr RW. Long-term inhibition of murine experimental autoimmune encephalomyelitis using CTLA-4-Fc supports a key role for CD28 costimulation. J Clin Invest. 1995. 95(6): p. 2783-9.

191. de Waal Malefyt R, Haanen J, Spits H, Roncarolo MG, te Velde A, Figdor C, Johnson $\mathrm{K}$, Kastelein R, Yssel H, and de Vries JE. Interleukin 10 (IL-10) and viral IL-10 strongly reduce antigen-specific human $T$ cell proliferation by diminishing the antigen-presenting capacity of monocytes via downregulation of class II major histocompatibility complex expression. The Journal of Experimental Medicine. 1991. 174(4): p. 915-924.

192. Panitch HS, Hirsch RL, Schindler J, and Johnson KP. Treatment of multiple sclerosis with gamma interferon: Exacerbations associated with activation of the immune system. Neurology. 1987. 37(7): p. 1097.

193. Semple BD, Kossmann T, and Morganti-Kossmann MC. Role of chemokines in CNS health and pathology: a focus on the CCL2/CCR2 and CXCL8/CXCR2 networks. Journal of Cerebral Blood Flow \& Metabolism. 2009. 30(3): p. 459-473.

194. Fanger NA, Voigtlaender D, Liu C, Swink S, Wardwell K, Fisher J, Graziano RF, Pfefferkorn LC, and Guyre PM. Characterization of expression, cytokine regulation, and effector function of the high affinity IgG receptor Fc gamma RI (CD64) expressed on human blood dendritic cells. J Immunol. 1997. 158(7): p. 3090-8.

195. Hill KE, Zollinger LV, Watt HE, Carlson NG, and Rose JW. Inducible nitric oxide synthase in chronic active multiple sclerosis plaques: distribution, cellular expression and association with myelin damage. J Neuroimmunol. 2004. 151(1-2): p. 171-9.

196. Redford EJ, Kapoor R, and Smith KJ. Nitric oxide donors reversibly block axonal conduction: demyelinated axons are especially susceptible. Brain. 1997. 120 ( Pt 12): p. 2149-57.

197. Cappiello MG, Sutterwala FS, Trinchieri G, Mosser DM, and Ma X. Suppression of IL-12 transcription in macrophages following Fcy receptor ligation. The Journal of Immunology. 2001. 166(7): p. 4498-4506.

198. Kahwaji J, Barker E, Pepkowitz S, Klapper E, Villicana R, Peng A, Chang R, Jordan $\mathrm{SC}$, and Vo AA. Acute Hemolysis After High-Dose Intravenous Immunoglobulin Therapy in Highly HLA Sensitized Patients. Clinical Journal of the American Society of Nephrology. 2009. 4(12): p. 1993-1997.

199. Hartmann G and Krieg AM. CpG DNA and LPS induce distinct patterns of activation in human monocytes. Gene therapy. 1999. 6(5): p. 893-903. 
200. de Waal Malefyt R, Abrams J, Bennett B, Figdor CG, and de Vries JE. Interleukin 10 (IL-10) inhibits cytokine synthesis by human monocytes: an autoregulatory role of IL10 produced by monocytes. Journal of Experimental Medicine. 1991. 174(5): p. 12091220.

201. Sutterwala FS, Noel GJ, Clynes R, and Mosser DM. Selective Suppression of Interleukin-12 Induction after Macrophage Receptor Ligation. The Journal of Experimental Medicine. 1997. 185(11): p. 1977-1985.

202. Gille C, Dreschers S, Spring B, Tarnok A, Bocsi J, Poets CF, and Orlikowsky TW. Differential modulation of cord blood and peripheral blood monocytes by intravenous immunoglobulin. Cytometry B Clin Cytom. 2012. 82(1): p. 26-34.

203. Murugaiyan G, Martin S, and Saha B. Levels of CD40 expression on dendritic cells dictate tumour growth or regression. Clin Exp Immunol. 2007. 149(1): p. 194-202.

204. te Velde AA, de Waal Malefijt R, Huijbens RJ, de Vries JE, and Figdor CG. $I L-10$ stimulates monocyte $F c$ gamma $R$ surface expression and cytotoxic activity. Distinct regulation of antibody-dependent cellular cytotoxicity by IFN-gamma, IL-4, and IL10. J Immunol. 1992. 149(12): p. 4048-52.

205. Crow AR, Song S, Freedman J, Helgason CD, Humphries RK, Siminovitch KA, and Lazarus AH. IVIg-mediated amelioration of murine ITP via FcgammaRIIB is independent of SHIP1, SHP-1, and Btk activity. Blood. 2003. 102(2): p. 558-60.

206. Andersson J, Skansen-Saphir U, Sparrelid E, and Andersson U. Intravenous immune globulin affects cytokine production in T lymphocytes and monocytes/macrophages. Clin Exp Immunol. 1996. 104 Suppl 1: p. 10-20.

207. Hohlfeld R. Immunologic factors in primary progressive multiple sclerosis. Mult Scler. 2004. 10 Suppl 1: p. S16-21; discussion S21-2.

208. Gonsette R. Combination therapy for multiple sclerosis. International MS Journal. 2004. 11: p. 10-21.

209. Hepburn AL, Mason JC, and Davies KA. Expression of Fcy and complement receptors on peripheral blood monocytes in systemic lupus erythematosus and rheumatoid arthritis. Rheumatology. 2004. 43(5): p. 547-554.

210. Bauer J, Bauer TM, Kalb T, Taga T, Lengyel G, Hirano T, Kishimoto T, Acs G, Mayer L, and Gerok W. Regulation of interleukin 6 receptor expression in human monocytes and monocyte-derived macrophages. Comparison with the expression in human hepatocytes. J Exp Med. 1989. 170(5): p. 1537-49.

211. Moniuszko M, Bodzenta-Lukaszyk A, and Dabrowska M. Oral glucocorticoid treatment decreases interleukin-10 receptor expression on peripheral blood leucocyte subsets. Clin Exp Immunol. 2009. 156(2): p. 328-35.

212. Sica A, Saccani A, Borsatti A, Power CA, Wells TN, Luini W, Polentarutti N, Sozzani S, and Mantovani A. Bacterial lipopolysaccharide rapidly inhibits expression of C-C chemokine receptors in human monocytes. J Exp Med. 1997. 185(5): p. 96974. 
213. Stapulionis R, Pinto Oliveira CL, Gjelstrup MC, Pedersen JS, Hokland ME, Hoffmann SV, Poulsen K, Jacobsen C, and Vorup-Jensen T. Structural Insight into the Function of Myelin Basic Protein as a Ligand for Integrin $\alpha M \beta 2$. The Journal of Immunology. 2008. 180(6): p. 3946-3956.

214. Geissmann F, Jung S, and Littman DR. Blood Monocytes Consist of Two Principal Subsets with Distinct Migratory Properties. Immunity. 2003. 19(1): p. 71-82.

215. Kazatchkine MD and Kaveri SV. Immunomodulation of Autoimmune and Inflammatory Diseases with Intravenous Immune Globulin. New England Journal of Medicine. 2001. 345(10): p. 747-755.

216. Nagayama H, Sato K, Kawasaki H, Enomoto M, Morimoto C, Tadokoro K, Juji T, Asano S, and Takahashi TA. IL-12 Responsiveness and Expression of IL-12 Receptor in Human Peripheral Blood Monocyte-Derived Dendritic Cells. The Journal of Immunology. 2000. 165(1): p. 59-66.

217. de Waal Malefyt R, Abrams J, Bennett B, Figdor CG, and de Vries JE. Interleukin 10(IL-10) inhibits cytokine synthesis by human monocytes: an autoregulatory role of IL-10 produced by monocytes. J Exp Med. 1991. 174(5): p. 1209-20.

218. Sellebjerg F, Hesse D, Limborg S, Lund H, Sondergaard H, Krakauer M, and Sorensen P. Dendritic cell, monocyte and T cell activation and response to glatiramer acetate in multiple sclerosis. Mult Scler. 2013. 19(2): p. 179-87.

219. Simpson JE, Newcombe J, Cuzner ML, and Woodroofe MN. Expression of monocyte chemoattractant protein-1 and other beta-chemokines by resident glia and inflammatory cells in multiple sclerosis lesions. J Neuroimmunol. 1998. 84(2): p. 23849.

220. McManus C, Berman JW, Brett FM, Staunton H, Farrell M, and Brosnan CF. MCP-1, MCP-2 and MCP-3 expression in multiple sclerosis lesions: an immunohistochemical and in situ hybridization study. J Neuroimmunol. 1998. 86(1): p. 20-9.

221. Van Der Voorn P, Tekstra J, Beelen RH, Tensen CP, Van Der Valk P, and De Groot CJ. Expression of $M C P-1$ by reactive astrocytes in demyelinating multiple sclerosis lesions. Am J Pathol. 1999. 154(1): p. 45-51.

222. Cella M, Sallusto F, and Lanzavecchia A. Origin, maturation and antigen presenting function of dendritic cells. Current Opinion in Immunology. 1997. 9(1): p. 10-16.

223. Comi G, Filippi M, and Wolinsky JS. European/Canadian multicenter, double-blind, randomized, placebo-controlled study of the effects of glatiramer acetate on magnetic resonance imaging--measured disease activity and burden in patients with relapsing multiple sclerosis. European/Canadian Glatiramer Acetate Study Group. Ann Neurol. 2001. 49(3): p. 290-7.

224. Tullman M and Lublin F. Combination therapy in multiple sclerosis. Current Neurology and Neuroscience Reports. 2005. 5(3): p. 245-248. 
225. Dhib-Jalbut S, Chen M, Henschel K, Ford D, Costello K, and Panitch H. Effect of combined IFNbeta-1 a and glatiramer acetate therapy on GA-specific T-cell responses in multiple sclerosis. Mult Scler. 2002. 8(6): p. 485-91.

226. Conway D and Cohen JA. Combination therapy in multiple sclerosis. Lancet Neurol. 2010. 9(3): p. 299-308.

227. Makretsov NA, Huntsman DG, Nielsen TO, Yorida E, Peacock M, Cheang MCU, Dunn SE, Hayes M, van de Rijn M, Bajdik C, and Gilks CB. Hierarchical Clustering Analysis of Tissue Microarray Immunostaining Data Identifies Prognostically Significant Groups of Breast Carcinoma. Clinical Cancer Research. 2004. 10(18): p. 6143-6151.

228. Alizadeh AA, Eisen MB, Davis RE, Ma C, Lossos IS, Rosenwald A, Boldrick JC, Sabet H, Tran T, and Yu X. Distinct types of diffuse large B-cell lymphoma identified by gene expression profiling. Nature. 2000. 403(6769): p. 503-511.

229. Gay F, Drye T, Dick G, and Esiri M. The application of multifactorial cluster analysis in the staging of plaques in early multiple sclerosis. Identification and characterization of the primary demyelinating lesion. Brain. 1997. 120(8): p. 14611483.

230. Graumann U, Reynolds R, Steck AJ, and Schaeren - Wiemers N. Molecular changes in normal appearing white matter in multiple sclerosis are characteristic of neuroprotective mechanisms against hypoxic insult. Brain pathology. 2003. 13(4): p. 554-573.

231. Axtell RC, de Jong BA, Boniface K, van der Voort LF, Bhat R, De Sarno P, Naves R, Han M, Zhong F, and Castellanos JG. Thelper type 1 and 17 cells determine efficacy of interferon-[beta] in multiple sclerosis and experimental encephalomyelitis. Nature medicine. 2010. 16(4): p. 406-412.

232. Thieblemont N, Weiss L, Sadeghi HM, Estcourt C, and Haeffner-Cavaillon N. CD14lowCD16high: A cytokine-producing monocyte subset which expands during human immunodeficiency virus infection. European Journal of Immunology. 1995. 25(12): p. 3418-3424.

233. Ancuta P, Rao R, Moses A, Mehle A, Shaw SK, Luscinskas FW, and Gabuzda D. Fractalkine preferentially mediates arrest and migration of CD16+ monocytes. The Journal of experimental medicine. 2003. 197(12): p. 1701-1707.

234. Kerschensteiner M, Gallmeier E, Behrens L, Leal VV, Misgeld T, Klinkert WE, Kolbeck R, Hoppe E, Oropeza-Wekerle R-L, and Bartke I. Activated human T cells, B cells, and monocytes produce brain-derived neurotrophic factor in vitro and in inflammatory brain lesions: a neuroprotective role of inflammation? The Journal of experimental medicine. 1999. 189(5): p. 865-870.

235. Streilein JW and Bergstresser PR. Langerhans cells: antigen presenting cells of the epidermis. Immunobiology. 1984. 168(3-5): p. 285-300. 
236. Toebak MJ, Gibbs S, Bruynzeel DP, Scheper RJ, and Rustemeyer T. Dendritic cells: biology of the skin. Contact Dermatitis. 2009. 60(1): p. 2-20.

237. Boros P, Gondolesi G, and Bromberg JS. High dose intravenous immunoglobulin treatment: Mechanisms of action. Liver Transplantation. 2005. 11(12): p. 1469-1480.

238. Ramakrishna C, Newo AN, Shen YW, and Cantin E. Passively administered pooled human immunoglobulins exert IL-10 dependent anti-inflammatory effects that protect against fatal HSV encephalitis. PLoS Pathog. 2011. 7(6): p. e1002071.

239. Tanguay S and Killion JJ. Direct comparison of ELISPOT and ELISA-based assays for detection of individual cytokine-secreting cells. Lymphokine Cytokine Res. 1994. 13(4): p. 259-63.

240. Kouwenhoven M, Özenci V, Teleshova N, Hussein Y, Huang Y-M, Eusebio A, and Link H. Enzyme-Linked Immunospot Assays Provide a Sensitive Tool for Detection of Cytokine Secretion by Monocytes. Clinical and Diagnostic Laboratory Immunology. 2001. 8(6): p. 1248-1257.

241. Takai T. Fc receptors and their role in immune regulation and autoimmunity. Journal of clinical immunology. 2005. 25(1): p. 1-18.

242. Jung T, Schauer U, Heusser C, Neumann C, and Rieger C. Detection of intracellular cytokines by flow cytometry. J Immunol Methods. 1993. 159(1-2): p. 197-207. 
Appendices 


\section{Appendix 1}

\section{Kurtzke Expanded Disability Status Scale (EDSS)[181]}

0.0 - Normal neurological exam (all grade 0 in all Functional System (FS) scores*).

1.0 - No disability, minimal signs in one FS* (i.e., grade 1).

1.5 - No disability, minimal signs in more than one FS* (more than 1 FS grade 1).

2.0 - Minimal disability in one FS (one FS grade 2, others 0 or 1).

2.5 - Minimal disability in two FS (two FS grade 2, others 0 or 1 ).

3.0 - Moderate disability in one FS (one FS grade 3, others 0 or 1) or mild disability in three or four FS (three or four FS grade 2, others 0 or 1) though fully ambulatory.

3.5 - Fully ambulatory but with moderate disability in one FS (one grade 3) and one or two FS grade 2; or two FS grade 3 (others 0 or 1) or five grade 2 (others 0 or 1).

4.0 - Fully ambulatory without aid, self-sufficient, up and about some 12 hours a day despite relatively severe disability consisting of one FS grade 4 (others 0 or 1), or combination of lesser grades exceeding limits of previous steps; able to walk without aid or rest some 500 meters.

4.5 - Fully ambulatory without aid, up and about much of the day, able to work a full day, may otherwise have some limitation of full activity or require minimal assistance; characterized by relatively severe disability usually consisting of one FS grade 4 (others or 1) or combinations of lesser grades exceeding limits of previous steps; able to walk without aid or rest some 300 meters.

5.0 - Ambulatory without aid or rest for about 200 meters; disability severe enough to impair full daily activities (e.g., to work a full day without special provisions); (Usual FS equivalents are one grade 5 alone, others 0 or 1 ; or combinations of lesser grades usually exceeding specifications for step 4.0).

5.5 - Ambulatory without aid for about 100 meters; disability severe enough to preclude full daily activities; (Usual FS equivalents are one grade 5 alone, others 0 or 1; or combination of lesser grades usually exceeding those for step 4.0).

6.0 - Intermittent or unilateral constant assistance (cane, crutch, brace) required to walk about 100 meters with or without resting; (Usual FS equivalents are combinations with more than two FS grade 3+).

6.5 - Constant bilateral assistance (canes, crutches, braces) required to walk about 20 meters without resting; (Usual FS equivalents are combinations with more than two FS grade 3+). 
7.0 - Unable to walk beyond approximately 5 meters even with aid, essentially restricted to wheelchair; wheels self in standard wheelchair and transfers alone; up and about in wheelchair some 12 hours a day; (Usual FS equivalents are combinations with more than one FS grade 4+; very rarely pyramidal grade 5 alone).

7.5 - Unable to take more than a few steps; restricted to wheelchair; may need aid in transfer; wheels self but cannot carry on in standard wheelchair a full day; May require motorized wheelchair; (Usual FS equivalents are combinations with more than one FS grade 4+).

8.0 - Essentially restricted to bed or chair or perambulated in wheelchair, but may be out of bed itself much of the day; retains many self-care functions; generally has effective use of arms; (Usual FS equivalents are combinations, generally grade 4+ in several systems).

8.5 - Essentially restricted to bed much of day; has some effective use of arm(s); retains some self-care functions; (Usual FS equivalents are combinations, generally 4+ in several systems). 9.0 - Helpless bed patient; can communicate and eat; (Usual FS equivalents are combinations, mostly grade $4+$ ).

9.5 - Totally helpless bed patient; unable to communicate effectively or eat/swallow; (Usual FS equivalents are combinations, almost all grade 4+).

10.0 - Death due to MS.

*Excludes cerebral function grade 1.

Note 1: EDSS steps 1.0 to 4.5 refer to patients who are fully ambulatory and the precise step number is defined by the Functional System score(s). EDSS steps 5.0 to 9.5 are defined by the impairment to ambulation and usual equivalents in Functional Systems scores are provided.

Note 2: EDSS should not change by 1.0 step unless there is a change in the same direction of at least one step in at least one FS. 


\section{Appendix 2}

\section{Fluorescent anti-human antibodies for flow cytometry}

\begin{tabular}{|l|l|l|l|l|}
\hline \multicolumn{1}{|c|}{$\begin{array}{c}\text { Cell surface } \\
\text { marker }\end{array}$} & Fluorophore & \multicolumn{1}{|c|}{ Clone } & $\begin{array}{c}\text { Species and } \\
\text { Isotype }\end{array}$ & Manufacturer \\
\hline CD11b & AF488 & ICRF44 & Mouse IgG1 & BDBiosciences \\
\hline CD3 & PE & UCHT1 & Mouse IgG1 & BDBiosciences \\
\hline CD14 & PerCPCy5,5 & M5E2 & Mouse IgG2a & BDBiosciences \\
\hline CD4 & APCH7 & RPA-T4 & Mouse IgG1 & BDBiosciences \\
\hline CD16 & V450 & 3 G8 & Mouse IgG1 & BDBiosciences \\
\hline CD40 & PE & 5 C3 & Mouse IgG1 & BDBiosciences \\
\hline CD45 & V500 & HI30 & Mouse IgG1 & BDBiosciences \\
\hline HLA-DR & APC & TU36 & Mouse IgG2b & BDBiosciences \\
\hline CD64 & Bio & 10.1 & Mouse IgG1 & BDBiosciences \\
\hline CD 192 (CCR2) & AF 647 & 48607 & Mouse IgG2b & BDBiosciences \\
\hline IgG 1 & AF488 & MOPC-21 & Mouse IgG1 & BDBiosciences \\
\hline IgG 1 & PE & MOPC-21 & Mouse IgG1 & BDBiosciences \\
\hline IgG 2a & PerCPCy5,5 & G155-178 & Mouse IgG2a & BDBiosciences \\
\hline IgG 1 & APCH7 & MOPC-21 & Mouse IgG1 & BDBiosciences \\
\hline IgG 1 & V450 & MOPC-21 & Mouse IgG1 & BDBiosciences \\
\hline IgG 1 & X500 & G155-178 & Mouse IgG2a & BDBiosciences \\
\hline IgG 2a & MOPC-31C & Mouse IgG1 & BDBiosciences \\
\hline IgG 1 & & Mouse IgG1 & BDBiosciences \\
\hline
\end{tabular}


Appendix 3

Anti-human ELISA antibodies

\begin{tabular}{|l|l|l|}
\hline Antibody type & Antibody name & Concentration \\
\hline IL-12 capture & Mouse anti-hIL-12p40 & $4 \mu \mathrm{g} / \mathrm{ml}$ \\
\hline IL-12 detection & Biotin mouse anti-hIL-12p40 & $0,25 \mu \mathrm{g} / \mathrm{ml}$ \\
\hline IL-6 capture & Mouse anti-hIL-6 & $1 \mu \mathrm{g} / \mathrm{ml}$ \\
\hline IL-6 detection & Biotin mouse anti-hIL-6 & $0,5 \mu \mathrm{g} / \mathrm{ml}$ \\
\hline IL-10 capture & Mouse anti-hIL-10 & $1 \mu \mathrm{g} / \mathrm{ml}$ \\
\hline IL-10 detection & Biotin mouse anti-hIL-10 & $1 \mu \mathrm{g} / \mathrm{ml}$ \\
\hline
\end{tabular}


Appendix 4

Fluorescents antibodies for intracellular cytokine staining

\begin{tabular}{|l|l|l|l|l|}
\hline \multicolumn{1}{|c|}{$\begin{array}{c}\text { Cell surface } \\
\text { marker }\end{array}$} & Fluorophore & \multicolumn{1}{|c|}{ Clone } & $\begin{array}{c}\text { Species and } \\
\text { Isotype }\end{array}$ & Manufacturer \\
\hline CD16 & PerCP & 3 G8 & Mouse IgG1 & Biolegend \\
\hline CD14 & BV570 & M5E2 & Mouse IgG2 & Biolegend \\
\hline IL-12 & PE & C11,5 & Mouse IgG1 & Biolegend \\
\hline IL-6 & APC & MQ2-13A5 & Rat IgG1 & Biolegend \\
\hline IL-10 & BV421 & JES3-907 & Rat IgG1 & Biolegend \\
\hline IgG & PerCP & MOPC-21 & Mouse IgG1 & Biolegend \\
\hline IgG & BV570 & MOPC-173 & Mouse IgG2 & Biolegend \\
\hline IgG & PE & MOPC-21 & Mouse IgG1 & Biolegend \\
\hline IgG & APC & RTK-2071 & Rat IgG1 & Biolegend \\
\hline IgG & BV421 & RTK2071 & Rat IgG1 & Biolegend \\
\hline
\end{tabular}




\section{Appendix 5 \\ PCR primers}

\section{CD40:}

Sense: AAGAAGCCAACCAATAAGGCC

Antisense: CGTCGGGAAAATTGATCTCC

64 bp Accession NM_001250.4

\section{CD86:}

Sense: GCGGCCTCGCAACTCTTATA

Antisense: TCTCTTTTCTTGGTCTGTTCACTCTC 75 bp Accession NM_175862.4

\section{HLA-DR:}

Sense: GCCAA CCTGG AAA TCAT GACA

Antisense: $\quad$ AGGG CTG TTTGT GAG CACA 87 bp Accession NM_019111.4

\section{IL-12p40:}

Sense: GACATTCAGTGTCAAAAGCAGCA

Antisense: CCTTGTTGTCCCCTCTGACTCT 100 bp Accession AF180563.1

IL-6:

Sense: $\quad$ GGT ACA TCC TCG ACG GCA TCT

Antisense: GTG CCT CTT TGC TGC TTT CAC 81 bp Accession NM_000600.3

IL-10:

Sense: GCCTAACATGCTTCGAGATC

Antisense: TGATGTCTGGGTCTTGGTTC 206 bp Accession NM_000572.2

$\beta$-actin (housekeeping gene):

Sense: GGATGCAGAAGGAGATCACTG

Antisense: CGATCCACACGGAGTACTTG 90 bp Accession NM_001101.3

Cyclophilin B (housekeeping gene):

Sense: CCAACGCAGGCAAAGACACCAA

Antisense: GCTCTCCACCTTCCGCACCA 131 bp Accession M60857.1 


\section{Appendix 6}

\section{Intracellular IL-12 detection}

a.

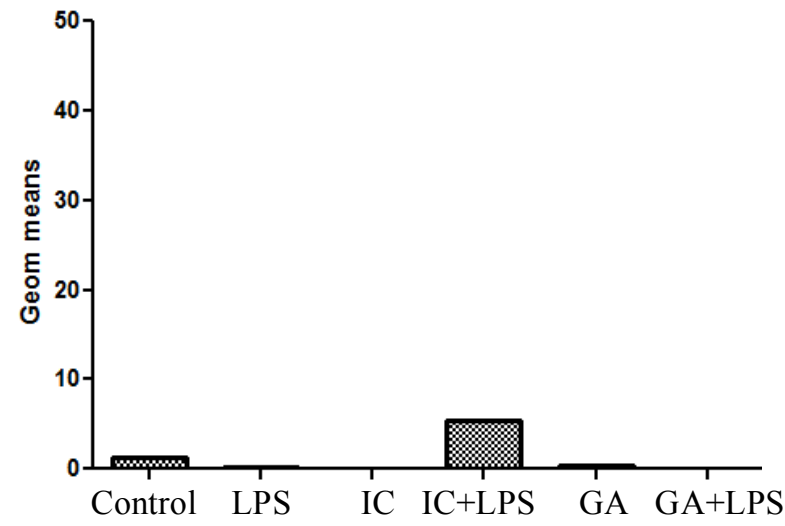

c.

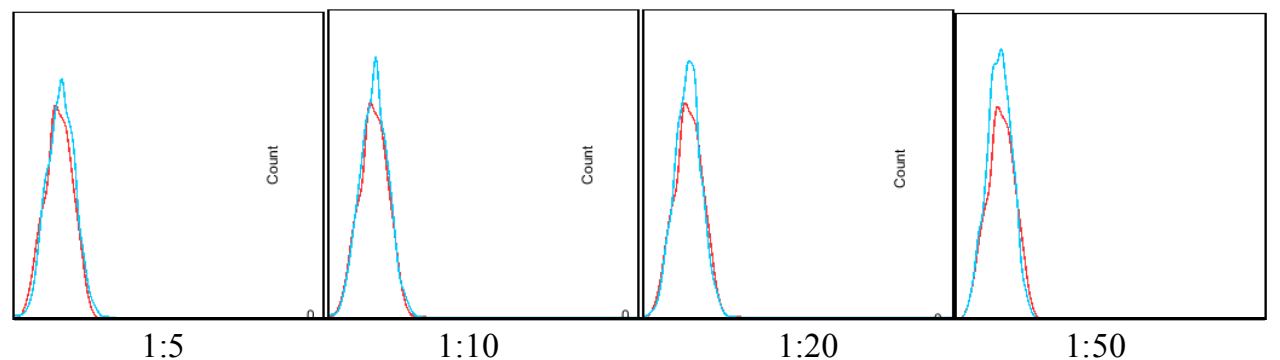

PE-conjugated anti-IL-12 antibody diltuions b.

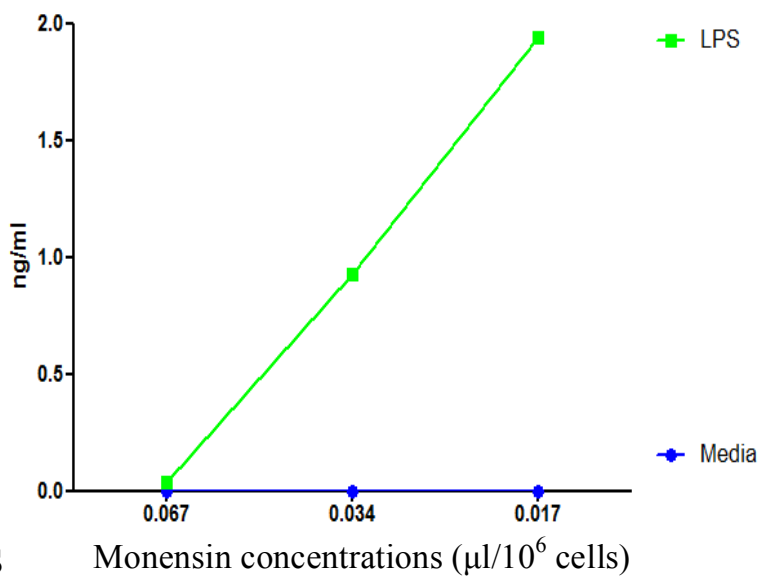

Monensin concentrations $\left(\mu 1 / 10^{6}\right.$ cells $)$ 


\section{Appendix 7}

\section{Buffers and solutions}

\section{PBS}

$\mathrm{NaCl} 145 \mathrm{mM}$

$\mathrm{Na} 2 \mathrm{HPO} 48.7 \mathrm{mM}$

$\mathrm{NaH} 2 \mathrm{PO} 41.3 \mathrm{Mm}$

In ddH2O, autoclaved sterilised

\section{MACS isolation buffer}

dPBS pH 7.2

Bovine serum albumin $0.5 \%$

EDTA $2 \mathrm{mM}$

Filter sterilised using a $0.22 \mu \mathrm{m}$ syringe driven filter

\section{Complete $\mathbf{T}$ cell media (CTCM)}

Dulbecco's Modified Eagle Medium 85.9\%

Fetal calf serum (FCS) 10\%

L-glutamate (200 mM) 1\%

Penicillin/Streptomycin 1\% (100 U/ml/10 mg/ml)

HEPES buffer $(1 \mathrm{M}) 1 \%$

$\beta$-Mecaptoethanol 0.1\%

Non-essential amino acids (10nM) 0.1\%

\section{FACS buffer $(\mathrm{v} / \mathrm{v})$}

FCS $2 \%$

Sodium azide $0.1 \%$

PBS 97.9\%

\section{ELISA capture buffer}

$\mathrm{Na} 2 \mathrm{HPO} 40.1 \mathrm{M}$

In ddH2O, adjusted to $\mathrm{pH} 9$ 


\section{ELISA development stop solution}

$\mathrm{H} 2 \mathrm{SO} 40.18 \mathrm{M}$

In $\mathrm{ddH} 2 \mathrm{O}$

\section{ELISA block solution}

FCS 5\%

In PBS

\section{MTT solution}

MTT $5 \mathrm{mg} / \mathrm{ml}$

(3-(4,5-dimethylthiazol-2-yl)-2.5-diphenyrtetrazolium bromide)

In dPBS

Filter sterilised using a $0.22 \mu \mathrm{m}$ syringe driven filter

\section{MTT solubiliser (w/v)}

SDS $10 \%$

Dimethylformamide $45 \%$

In $\mathrm{ddH} 2 \mathrm{O}$, adjusted to $\mathrm{pH} 4.5$ with acetic acid 


\section{Appendix 8 \\ Gating strategies for ex vivo isolated total monocytes}

a)

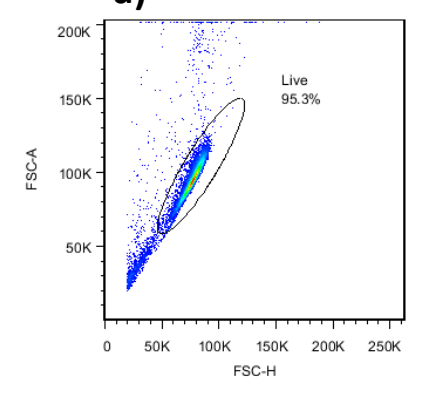

b)
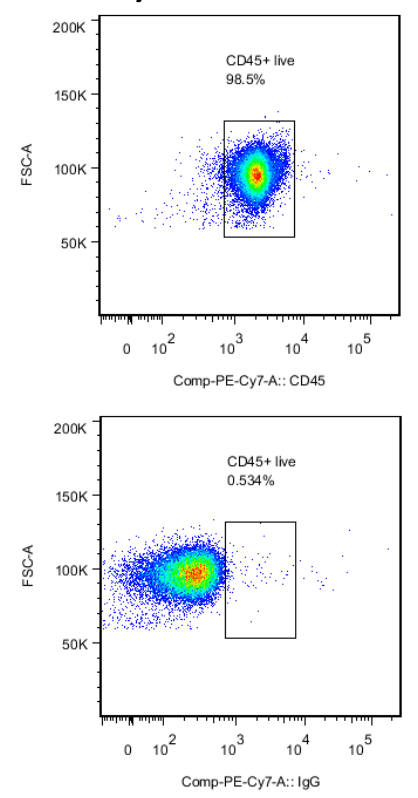

c)
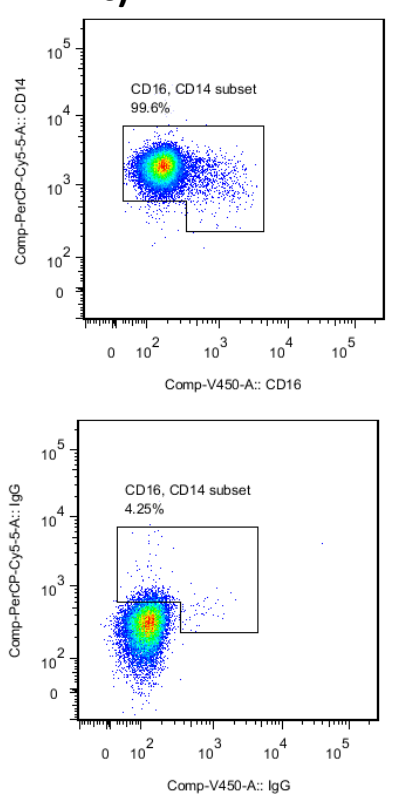

d)
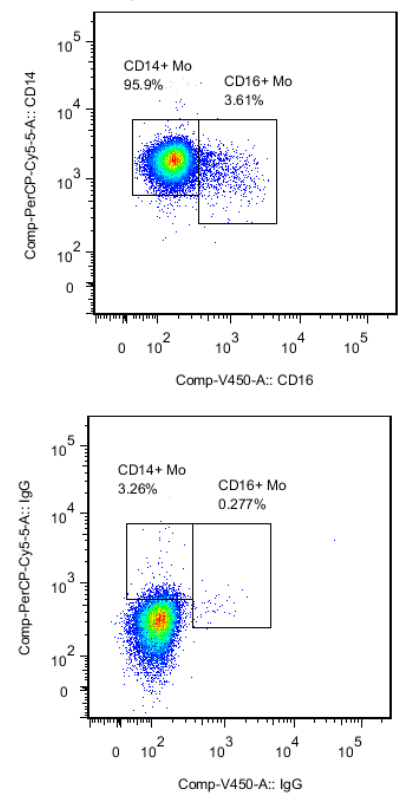

Appendix 8. Gating strategies for ex vivo isolated total monocytes.

Total monocyte populations were isolated from PBMC using CD14 microbeads. Isolated monocytes were stained with anti-CD antibodies and acquired on flow cytometry. Shown are examples of dot plots from one experiment with healthy monocytes. a) Isolated total monocytes were gated on live single cells to remove debris and doublets. b) Live single cells were then gated on $\mathrm{CD} 45^{+}$populations to select leucocytes. Shown are positively stained $\mathrm{CD} 45^{+}$populations and IgG controls below. c) Total monocyte populations were then gated from $\mathrm{CD}_{4} 5^{+}$live single cells, based on the expression of markers CD14 and CD16. Shown are positively stained $\mathrm{CD} 14^{+} \mathrm{CD} 16^{+}$populations and $\mathrm{IgG}$ controls below. d) $\mathrm{CD} 14^{+}$and $\mathrm{CD} 16^{+}$monocyte subsets were also gated from $\mathrm{CD} 45^{+}$live single cells. Shown are positively stained $\mathrm{CD} 14^{++} \mathrm{CD} 16^{-}$and $\mathrm{CD} 14^{+} \mathrm{CD} 16^{+}$populations and $\mathrm{IgG}$ controls below. 


\section{Appendix 9 \\ Gating strategies for experiments on cultured \\ total monocytes and co-cultured subsets}

a)

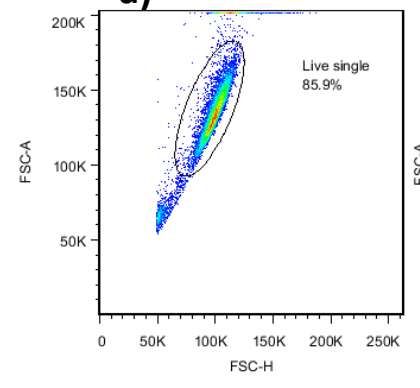

b)
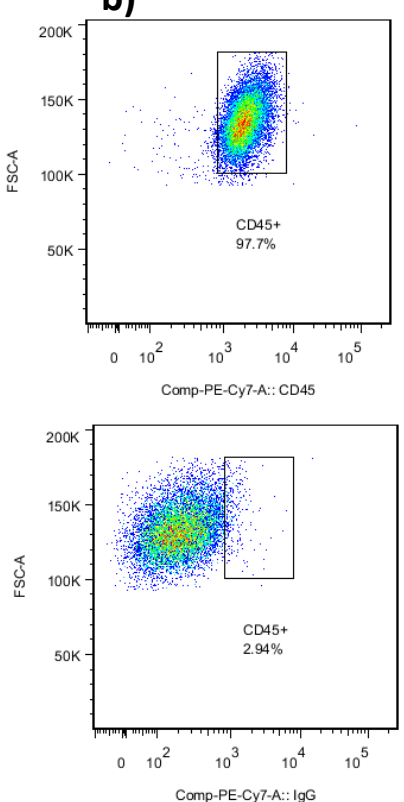

c)
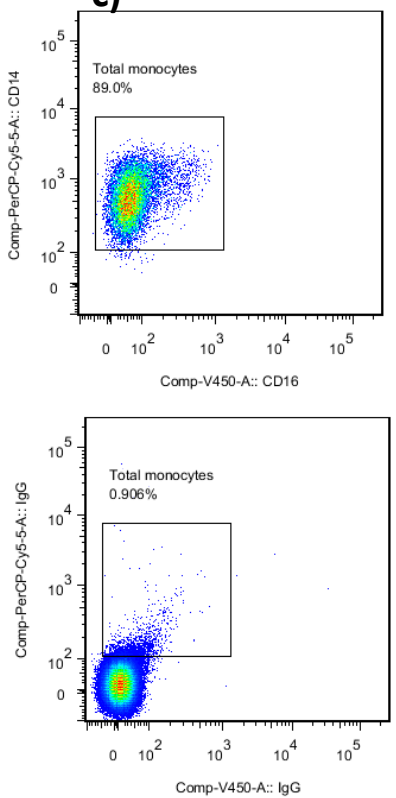

d)

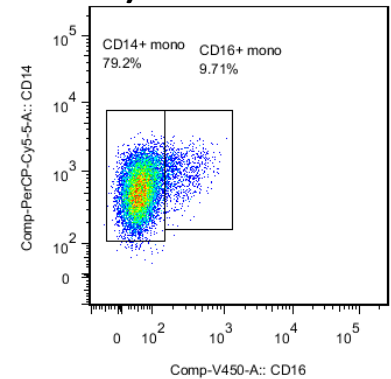

Comp-V450-A:: CD16

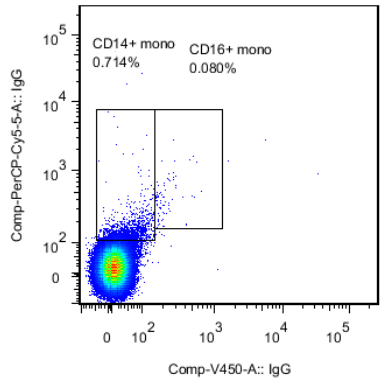

Appendix 9. Gating on cultured total monocytes and co-cultured $\mathrm{CD}^{+}{ }^{+}$and $\mathrm{CD}^{+}{ }^{+}$subsets.

Total monocyte populations were isolated from PBMC using CD14 microbeads. Isolated total monocytes were primed with IFN $\gamma$ for 16 hours, cultured in media for 24 hours, stained with anti-CD antibodies and acquired on flow cytometry. Shown are examples of dot plots from one experiment with healthy monocytes. a) Isolated total monocytes were gated on live single cells to remove debris and doublets. b) Live single cells were then gated on $\mathrm{CD} 45^{+}$populations to select leucocytes. Shown are positively stained $\mathrm{CD}^{+} 5^{+}$populations and $\mathrm{IgG}$ controls below. c) Total monocyte populations were then gated from $\mathrm{CD}^{+} 5^{+}$live single cells, based on the expression of markers CD14 and CD16. Shown are positively stained $\mathrm{CD} 14^{+} \mathrm{CD} 16^{+}$populations and $\mathrm{IgG}$ controls below. d) $\mathrm{CD} 14^{+}$and $\mathrm{CD} 16^{+}$monocyte subsets were also gated from $\mathrm{CD} 45^{+}$live single cells. Shown are positively stained $\mathrm{CD} 14^{++} \mathrm{CD} 16^{-}$and $\mathrm{CD} 14^{+} \mathrm{CD} 16^{+}$populations and $\mathrm{IgG}$ controls below. 


\section{Appendix 10 \\ Gating strategies for experiments \\ on isolated $\mathrm{CD}_{14}^{+}$monocytes (individually cultured)}

a)

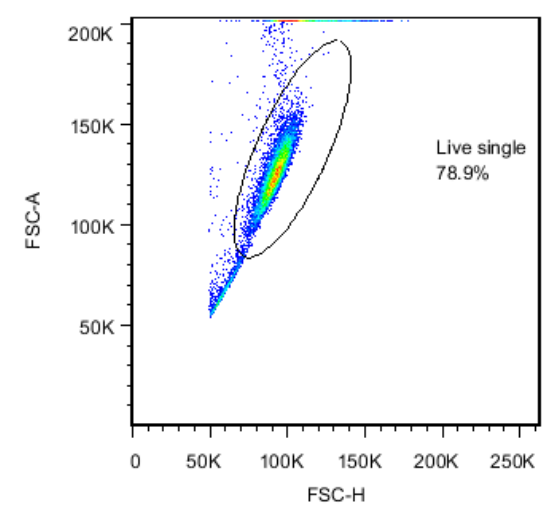

b)
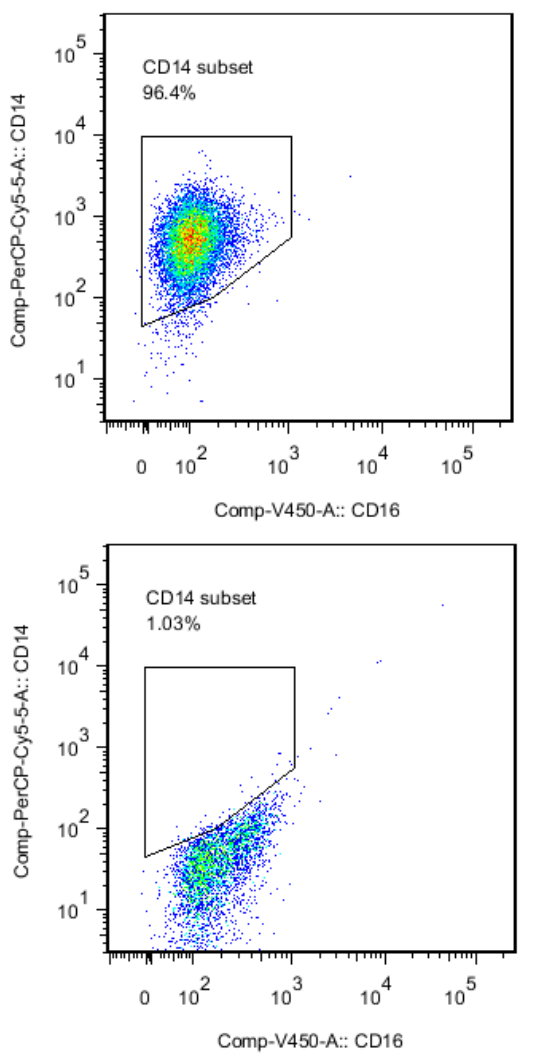

Appendix 10. Gating on individually cultured $\mathrm{CD}^{+}{ }^{+}$monocytes.

$\mathrm{CD} 14^{+}$monocyte populations were isolated from PBMC using CD14 microbeads after depletion of $\mathrm{CD} 15^{+}$ neutrophils, $\mathrm{CD} 56^{+} \mathrm{NK}$ cells and $\mathrm{CD} 16^{+}$monocytes. Isolated $\mathrm{CD} 14^{+}$monocytes were either stained with fluorophores to assess their purity on flow cytometry, or cultured and treated for further experiments with subsequent flow cytometry. Shown are examples of dot plots from one experiment with healthy monocytes.

a) Isolated total monocytes were acquired on flow cytometry and gated on live single cells to remove debris and doublets. b) $\mathrm{CD}_{14}{ }^{+}$monocyte subsets were then gated from live single cells, based on the expression of markers $\mathrm{CD} 14$ and CD16. Shown are positively stained populations and $\mathrm{IgG}$ controls below. 


\section{Appendix 11 \\ Gating strategies for experiments on isolated $\mathrm{CD}^{+}{ }^{+}$monocytes (individually cultured)}

a)

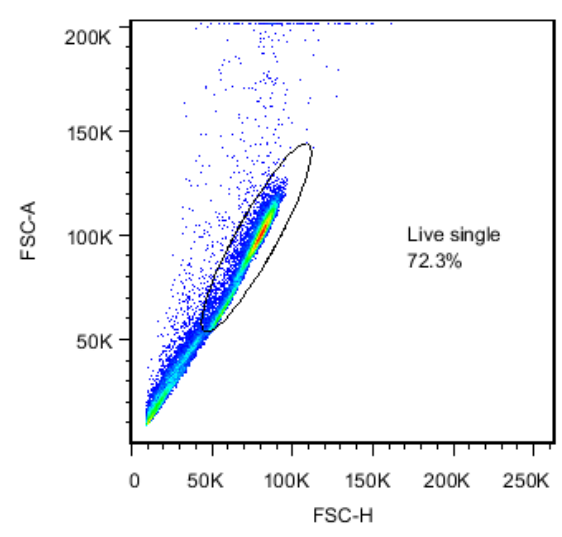

b)
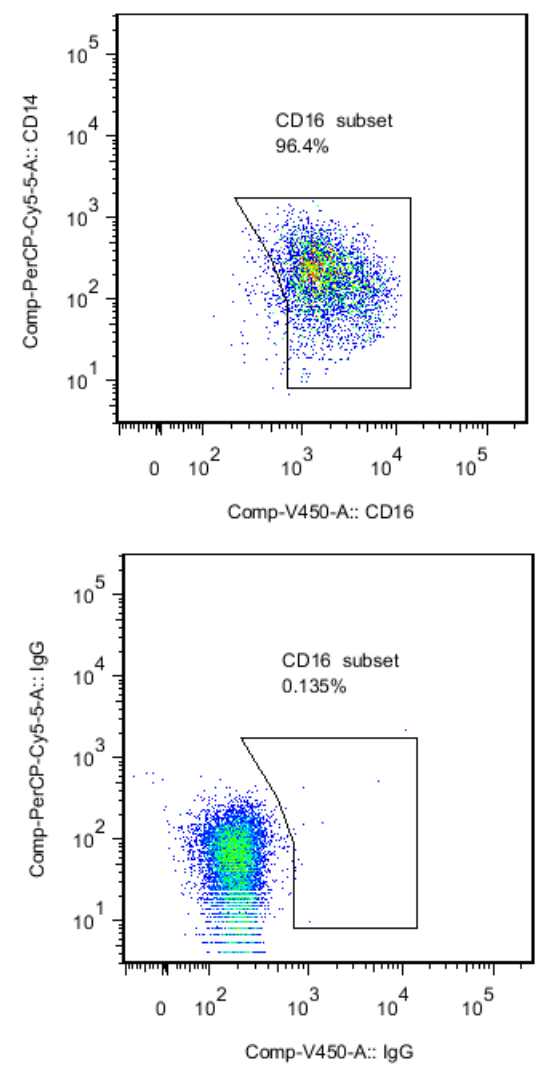

\section{Appendix 11. Gating on individually cultured $\mathrm{CD}^{+}{ }^{+}$monocytes.}

$\mathrm{CD} 16^{+}$monocyte subsets were isolated from PBMC using CD16 microbeads after depletion of $\mathrm{CD} 15^{+} \mathrm{CD} 16^{+}$ neutrophils and $\mathrm{CD} 56^{+} \mathrm{CD} 16^{+} \mathrm{NK}$ cells. Isolated $\mathrm{CD} 16^{+}$monocytes were either stained with fluorophores to assess their purity on flow cytometry, or cultured and treated for further experiments with subsequent flow cytometry. Shown are examples of dot plots from one experiment with healthy monocytes.

a) Isolated total monocytes were acquired on flow cytometry and gated on live single cells to remove debris and doublets. b) $\mathrm{CD} 16^{+}$monocyte subsets were then gated from live single cells, based on the expression of markers $\mathrm{CD} 14$ and CD16. Shown are positively stained populations and $\mathrm{IgG}$ controls below. 